\title{
Rhodium-Catalyzed Aerobic Decomposition of 1,3-Diaryl-2-diazo-1,3-diketones: Mechanistic Investigation and Application to the Synthesis of Benzils \\ Jia-Liang Zhu* and Yi-Ting Tsai
}

Department of Chemistry, National Dong-Hwa University, Hualien 974, Taiwan, R.O.C.

E-mail: jlzhu@gms.ndhu.edu.tw

\section{Supporting Information}

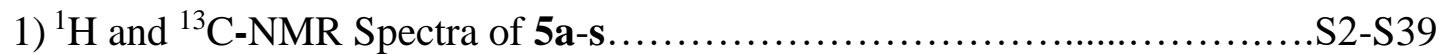

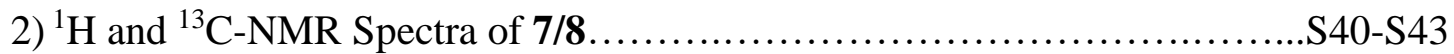

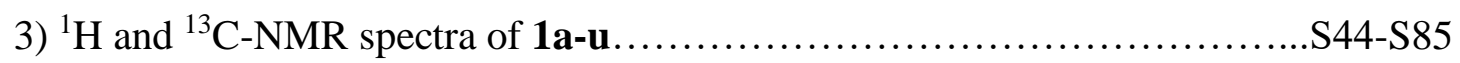

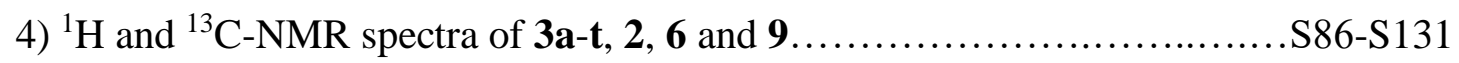

5) ${ }^{1} \mathrm{H}$ and ${ }^{13} \mathrm{C}-\mathrm{NMR}$ spectra of ${ }^{13} \mathrm{C}-5 \mathbf{b},{ }^{13} \mathrm{C}-\mathbf{1 b}$ and ${ }^{13} \mathrm{C}-3 \mathbf{b} \ldots \ldots \ldots \ldots \ldots . . . . . . . .5132-\mathrm{S} 137$

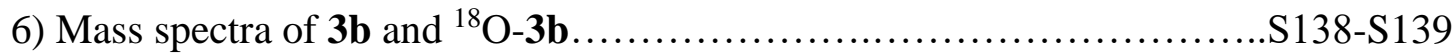

7) The ${ }^{1} \mathrm{H}$ NMR spectra of Ar- and $\mathrm{O}_{2}$-purged $\mathrm{Rh}_{2}(\mathrm{OAc})_{4}$ in $\mathrm{CDCl}_{3} \ldots \ldots \ldots \ldots . . . . . \mathrm{S} 140$

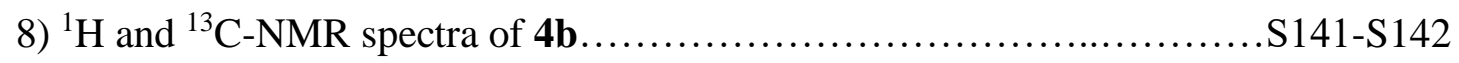

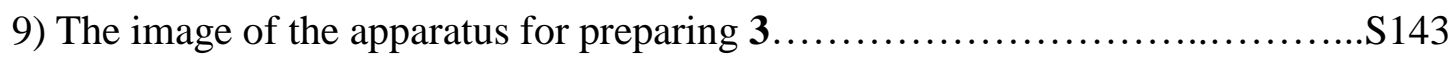

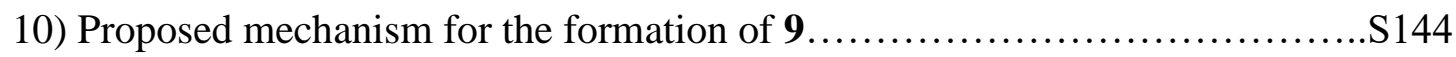




\section{1) ${ }^{1} \mathrm{H}$ and ${ }^{13} \mathrm{C}$-NMR Spectra of 5a-s}

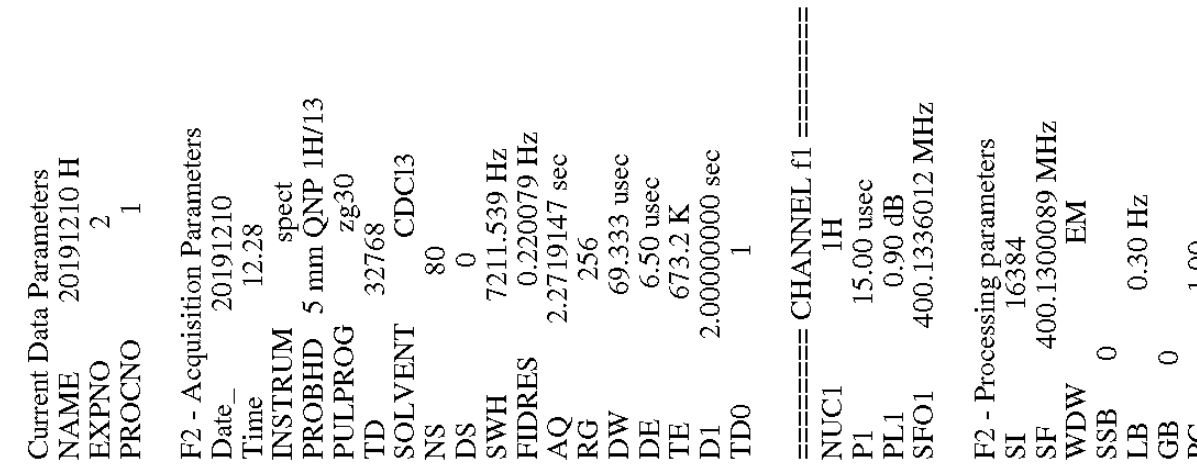<smiles>O=C(CC(=O)c1ccccc1-c1ccccc1)c1ccccc1</smiles>

$5 a$

$9 z \angle 6{ }^{\circ}{ }^{\circ}$

$S 09 Z^{\circ} L$

ZIEE: $L$

OOSE $L$

$889 \varepsilon L$

I6LE $L$ -

I68 $\varepsilon^{\circ} L$

$\varepsilon 66 \varepsilon\llcorner\cdot$

$\varepsilon \varepsilon Z \nabla^{\circ} L$

$\tau \nabla \varepsilon \nabla^{\circ} L$

29St $L$

ZL6T:L

$200 S^{\circ} L$

Z80S'L

OIIS $L$

$8 I E S^{\circ} L-$

SISS: $L$

$6 t S S^{\circ} L$

ZOLS L

$\tau \varepsilon L S^{\circ} L$

$\angle Z I 8^{\circ} L$

ISI $8^{\circ} L-$

$8 I \varepsilon 8 . L$

$\left[t \varepsilon 8^{\circ} L\right.$

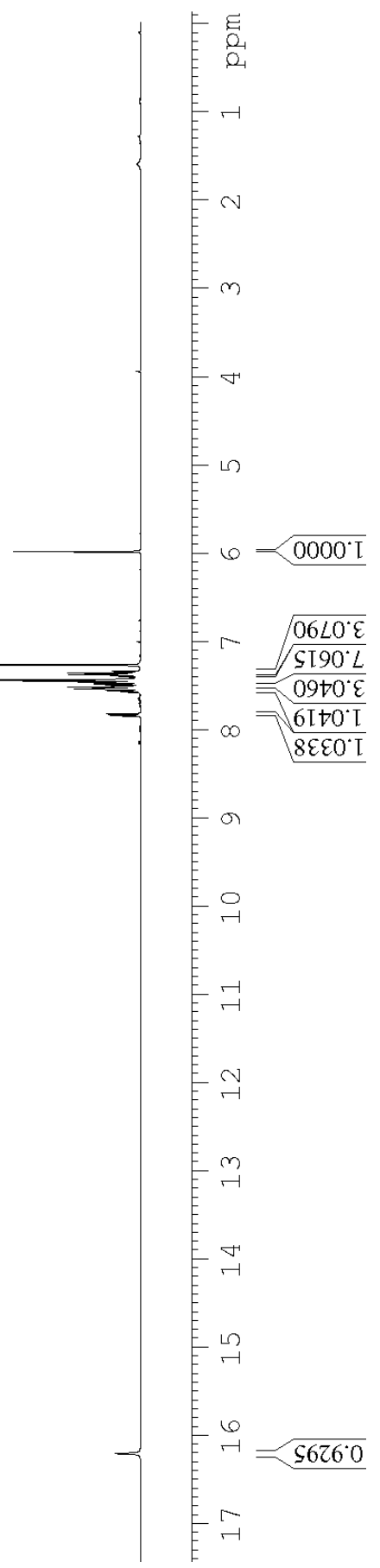




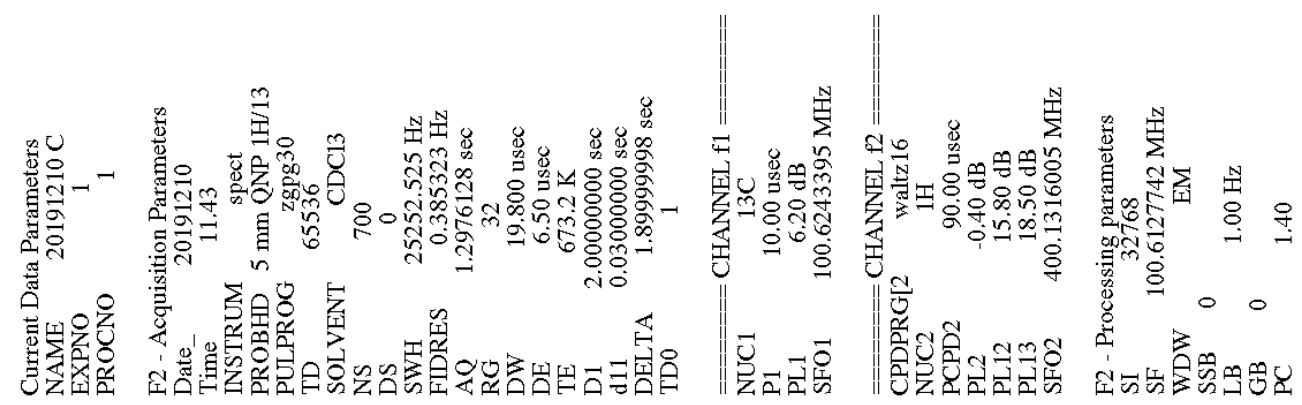

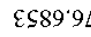

$0 \varepsilon 00 . \mathrm{LL}$

$\$ 90 Z^{\circ} \mathrm{LL}$

ZOZE'LL-<smiles>O=C(CC(=O)c1ccccc1-c1ccccc1)c1ccccc1</smiles>

$5 a$

LSZE:66

It68.9ZI

ZtES'LZI

I9SS $L Z I$

8SET $8 Z \mathrm{I}$

$016 t^{\circ} 8 \mathrm{ZI}$

It000\% 6 I

$68 \mathrm{tI} \cdot 6 \mathrm{ZI}$

I $T L^{\circ} O E$ I

†888 0 \& I

90ZI'ZEI -

$8 \varepsilon 88^{\circ} \downarrow \varepsilon \mathrm{I}$

วऽES $9 \mathcal{E}$ I

0 IL6 OtI

$\varsigma \mathcal{C}$ 'I†I

oIst'28I

$69 \angle \varepsilon^{\circ} 06 \mathrm{I}$

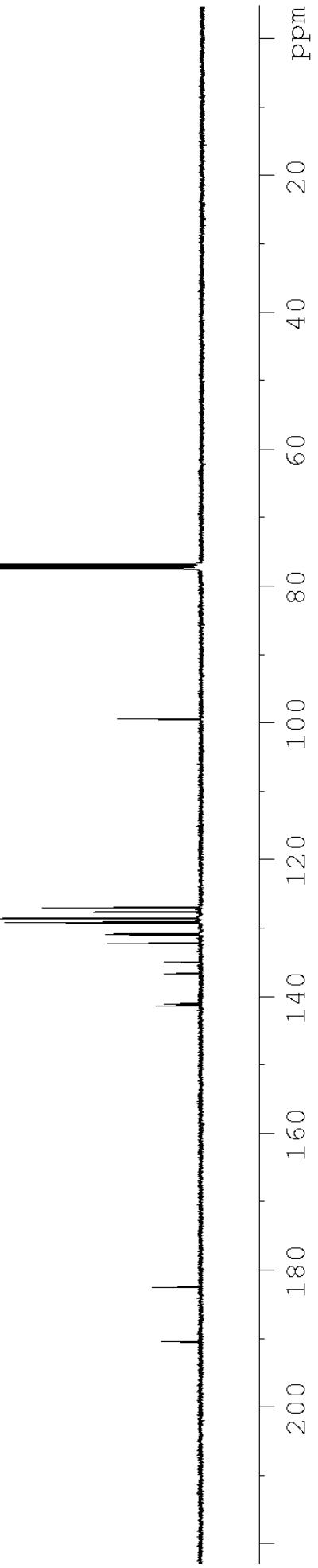




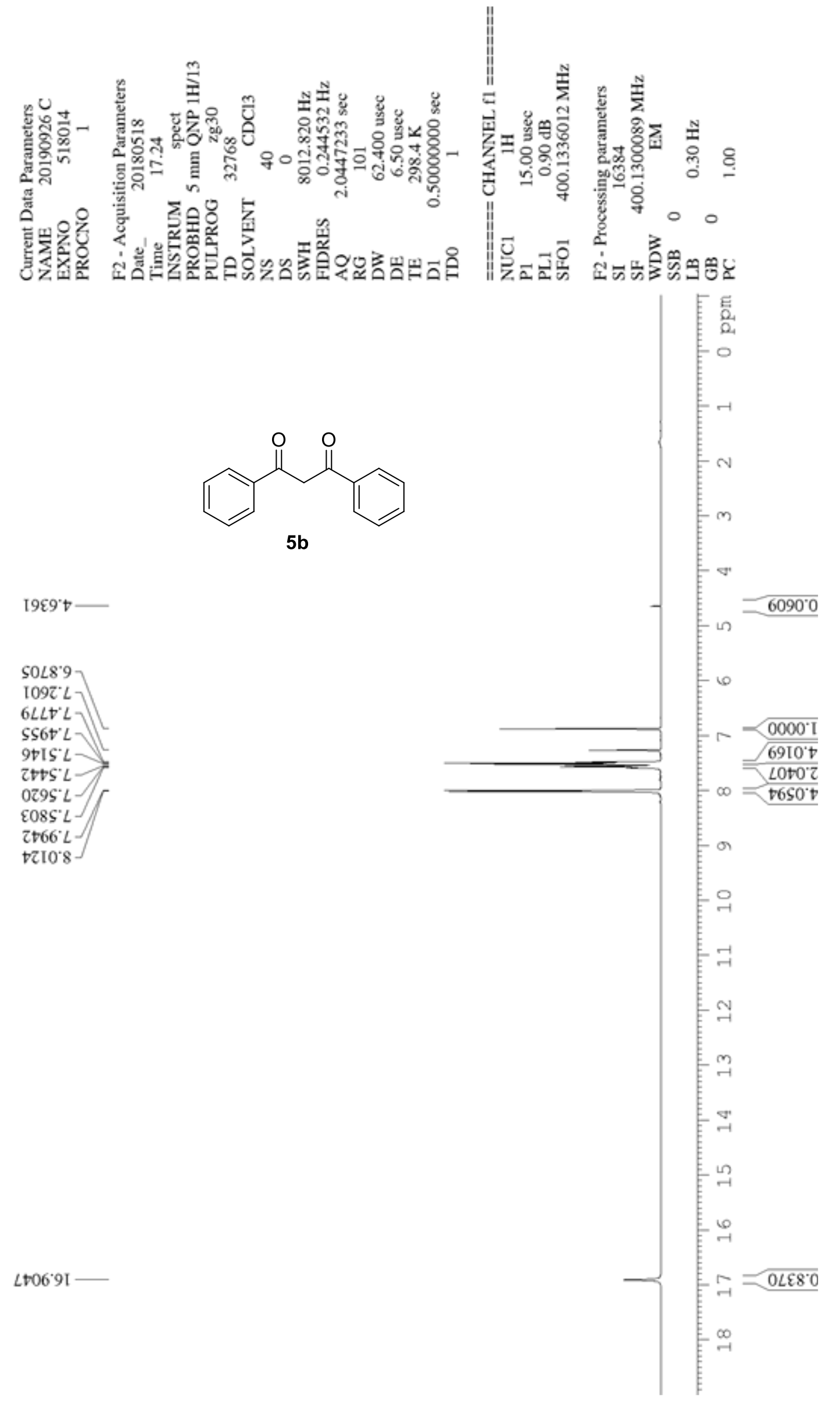



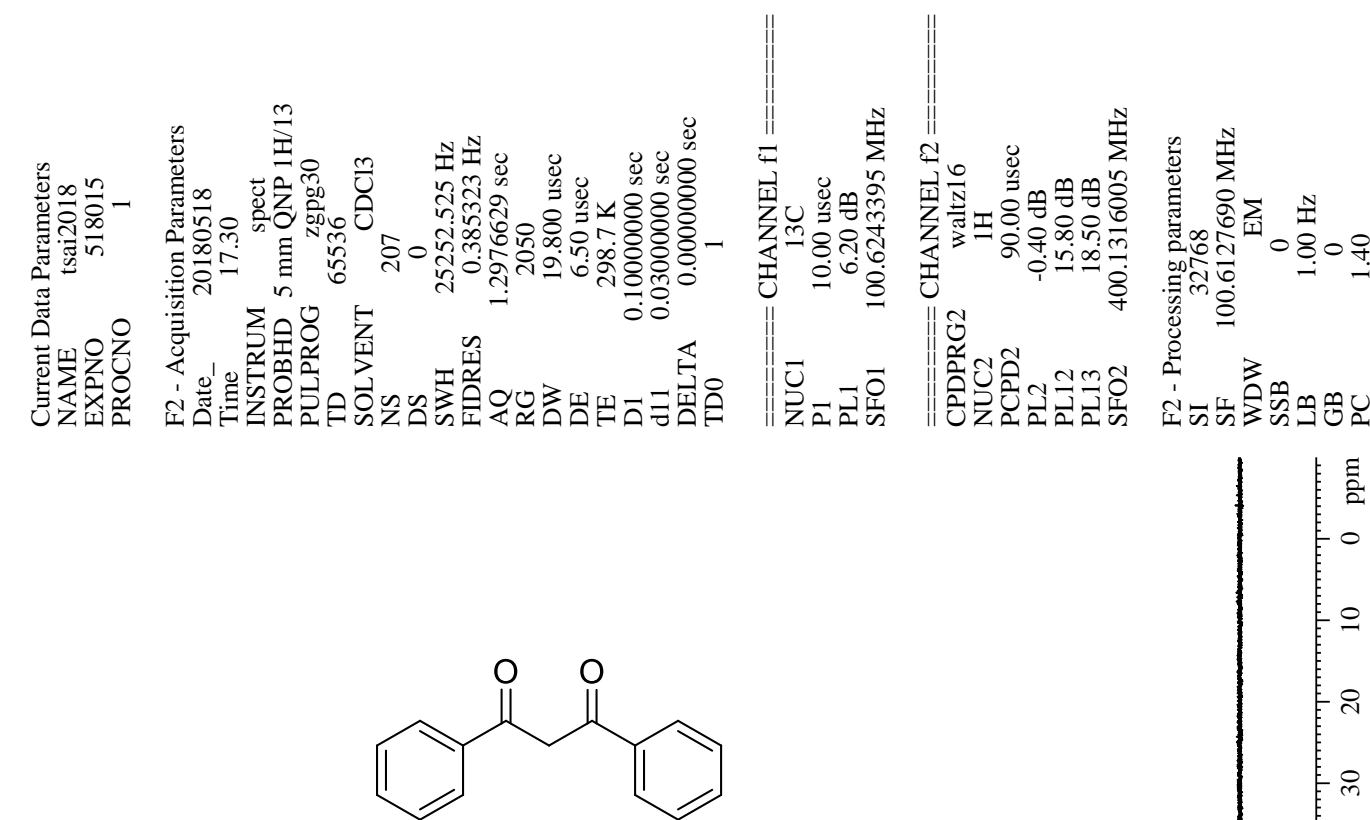

5b

$\varepsilon 6 \subseteq L^{\circ} 9 L$

$\angle 9 L 0^{\circ} L L$

I $6 \angle Z: L L$

$6+6 \varepsilon \cdot L L$

†LLI'E6

E96 I' LZI

†80L' $8 Z \mathrm{I}$

$\angle Z 8 T^{\circ} Z \varepsilon$

It $9 S^{\circ} \subseteq$ I I

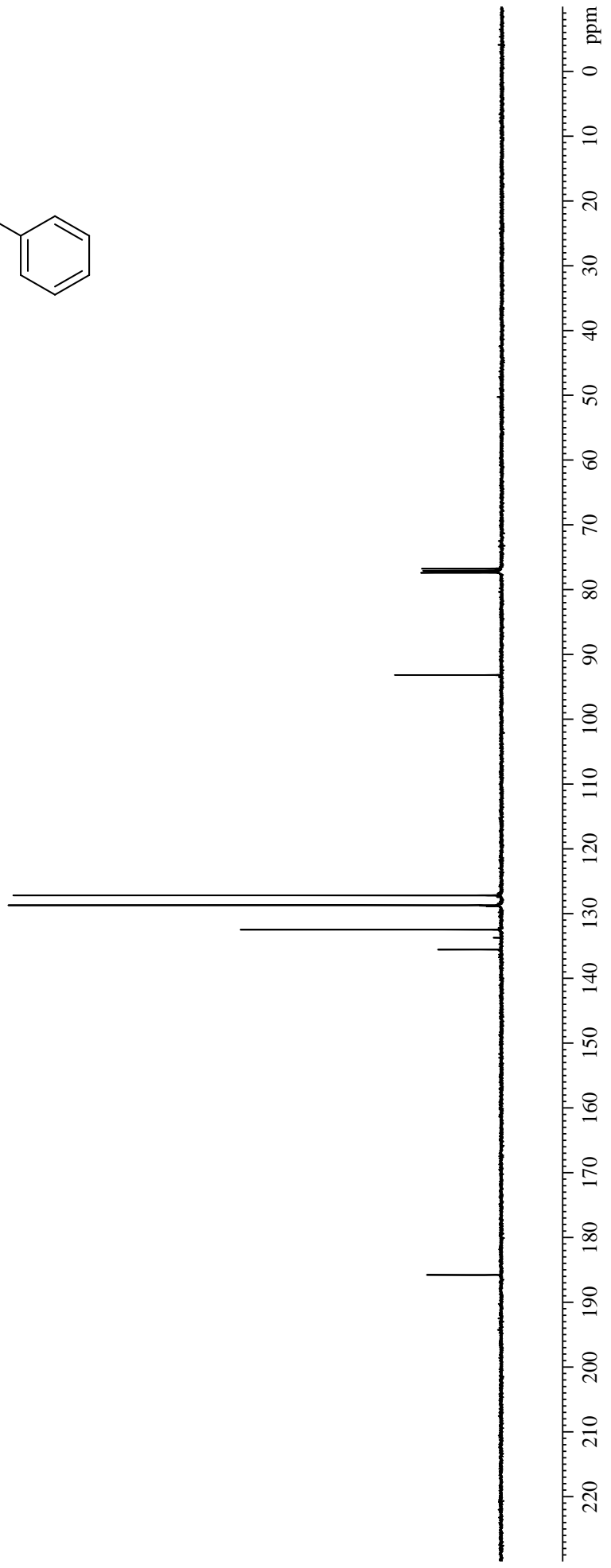

Z†8L'S8I 


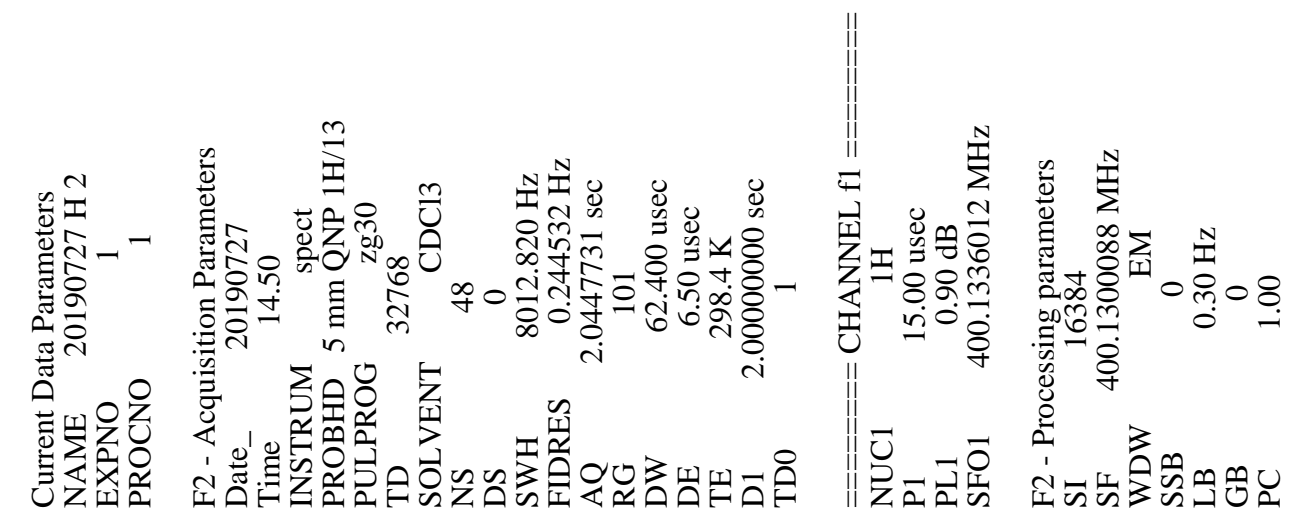<smiles>Cc1ccccc1C(=O)CC(=O)c1ccccc1</smiles>
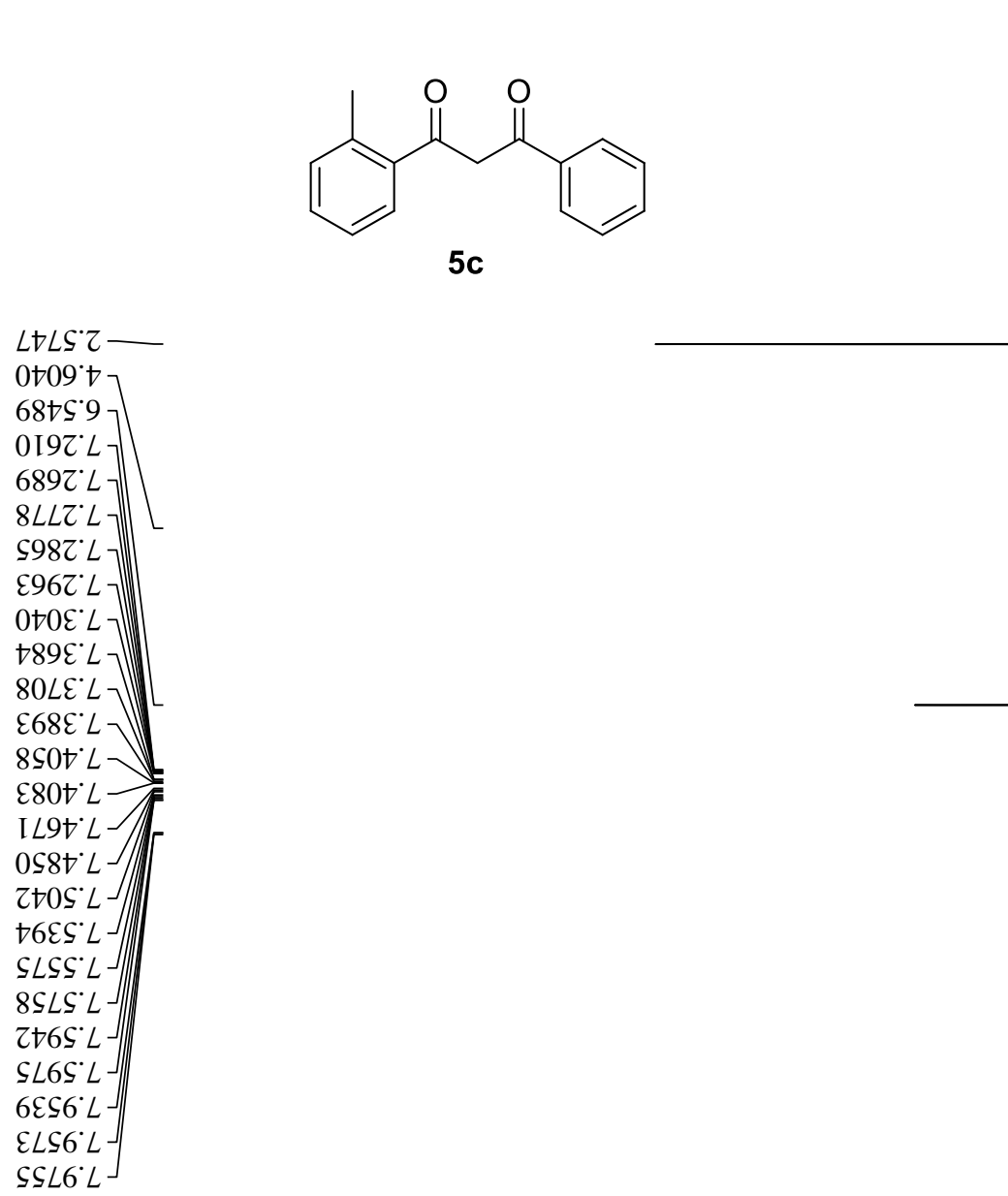


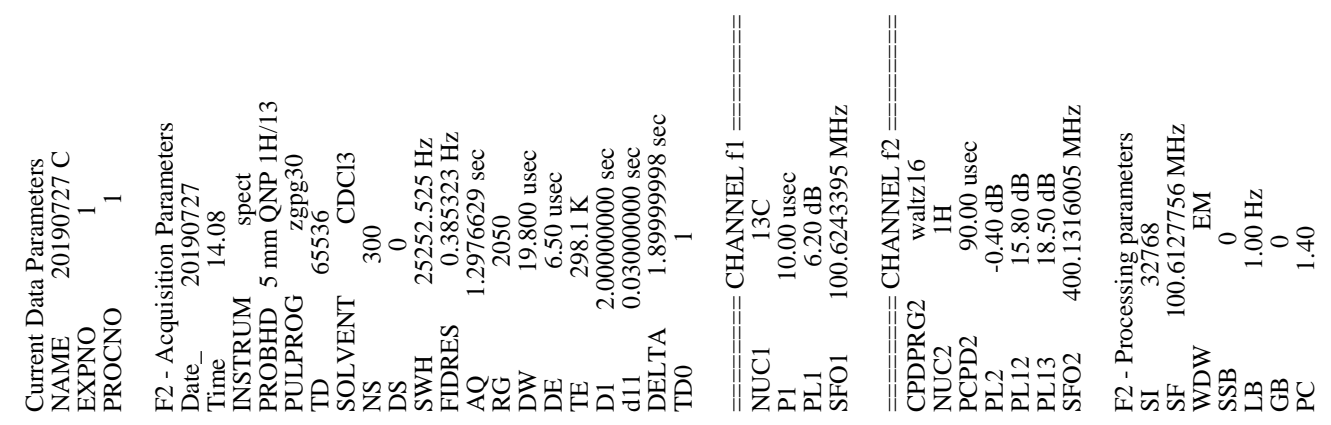

LI99.0Z -

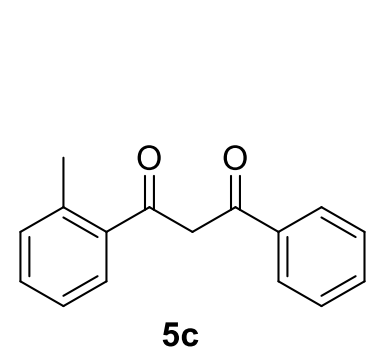

[989.9L

I $000^{\circ} L L$

EIZE'LL

E9zZ:L6

ZL8L'SZI

t90 [ $\angle Z I]$

[s 58.821

SS $59^{\circ} 8 Z \mathrm{I}$
$\angle 6 \mathrm{I} \angle{ }^{\circ} 0 \mathrm{EI}$

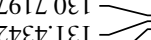

IITtZEI

$00 \angle Z$ 'SEI

$8+\varepsilon 0^{\circ} 9 \varepsilon \mathrm{I}$

$5 c$

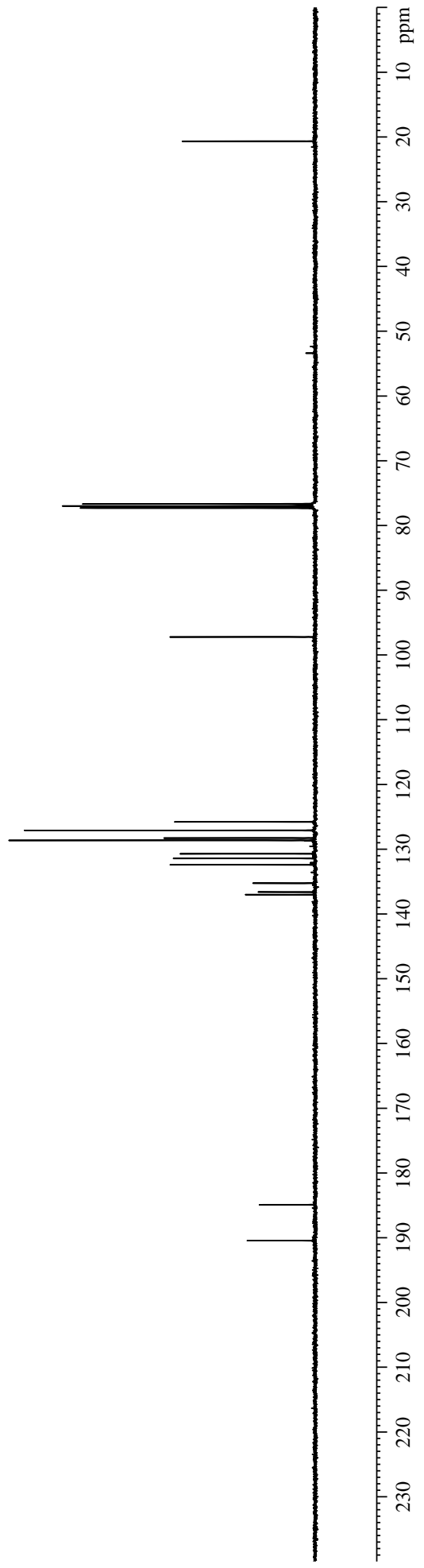

2I06 $58 \mathrm{I}$

EEtt 06I

言

SSZO LEI 


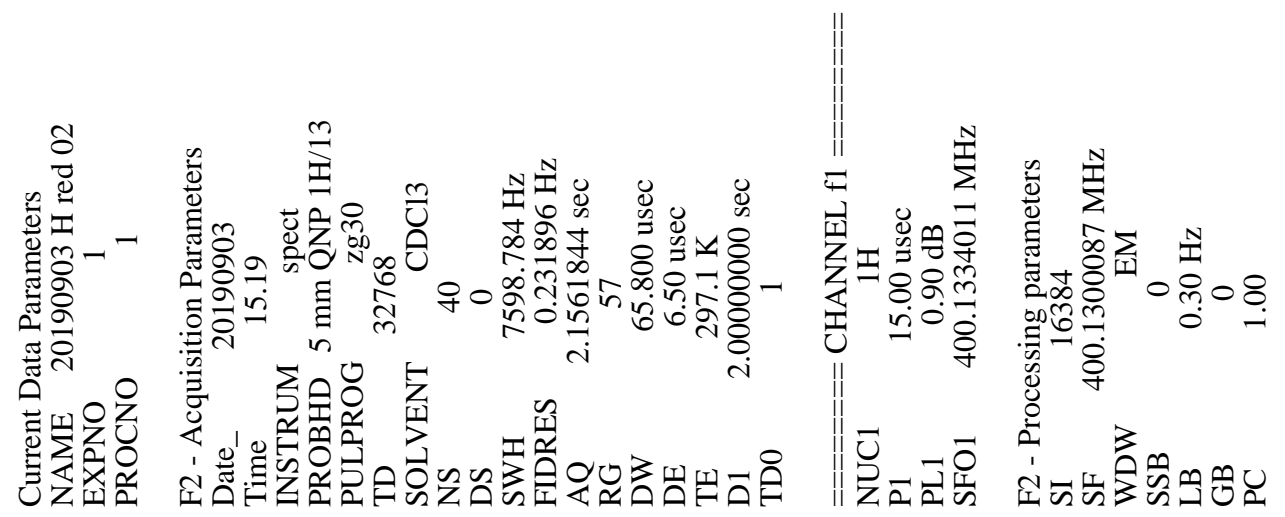<smiles>CCCCCCC(=O)c1ccccc1Cl</smiles>
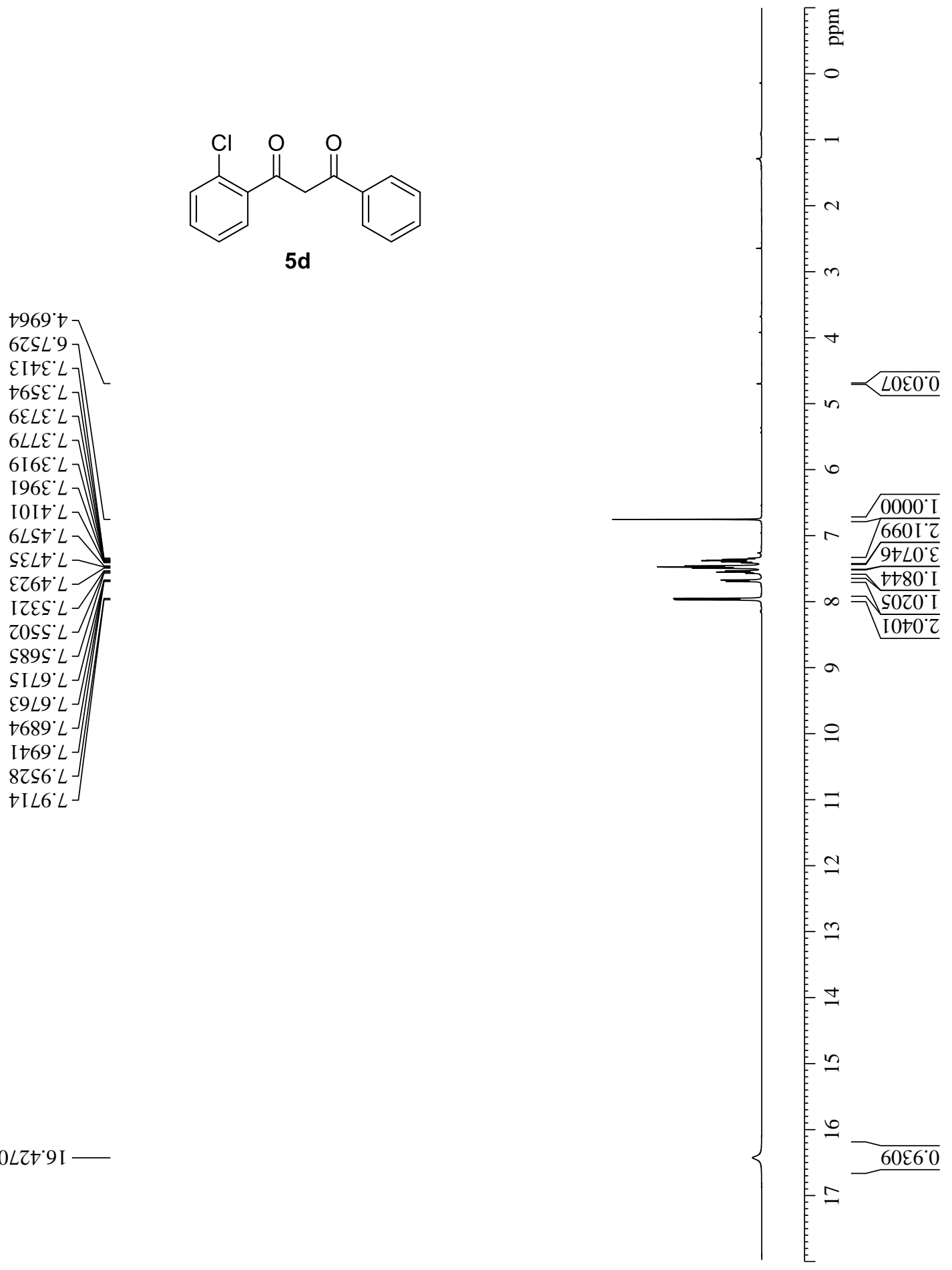


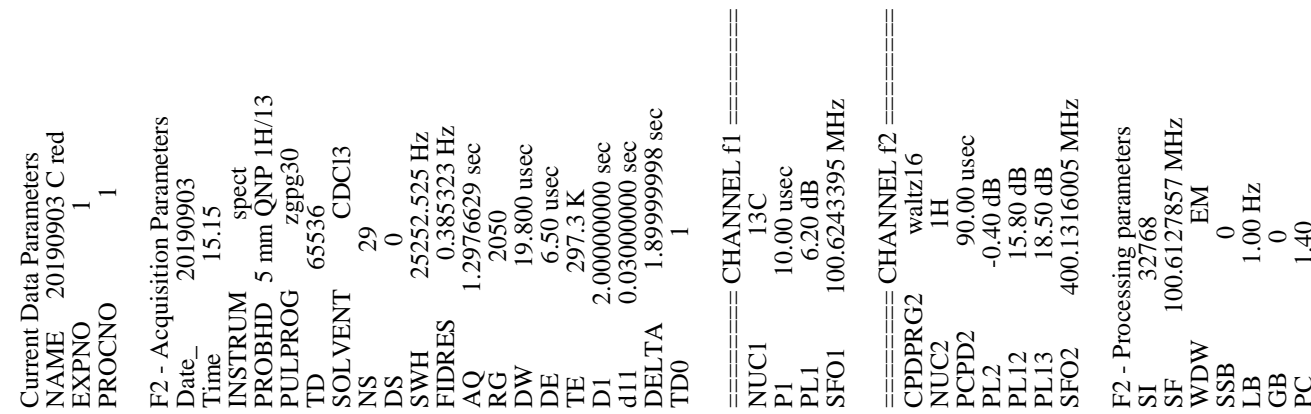<smiles>O=C(CC(=O)c1ccccc1Cl)c1ccccc1</smiles>

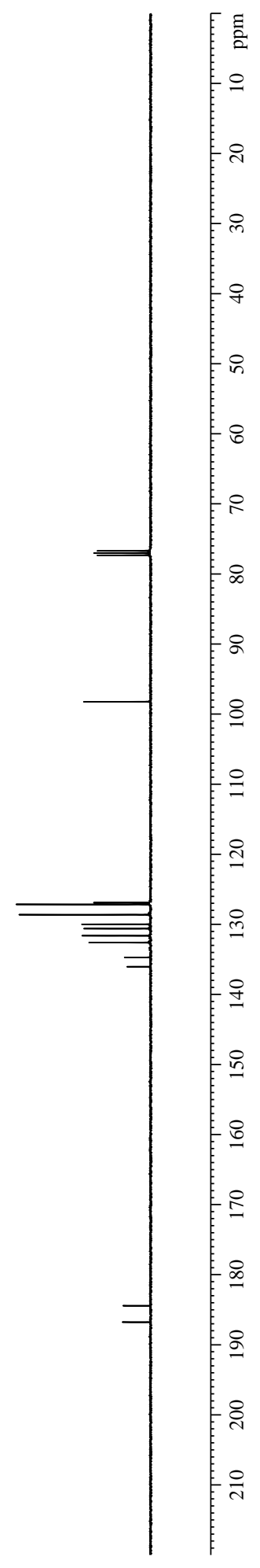

I $\angle 89^{\circ} 9 L$

$\angle \supset 00^{\circ} \angle L$

ZEZE $L L$

ZIZZ: 86

$5 d$

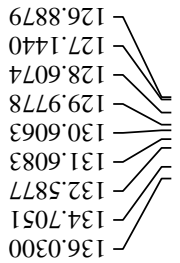

$6 S$ It $\downarrow 8 \mathrm{I}$

ह 은 


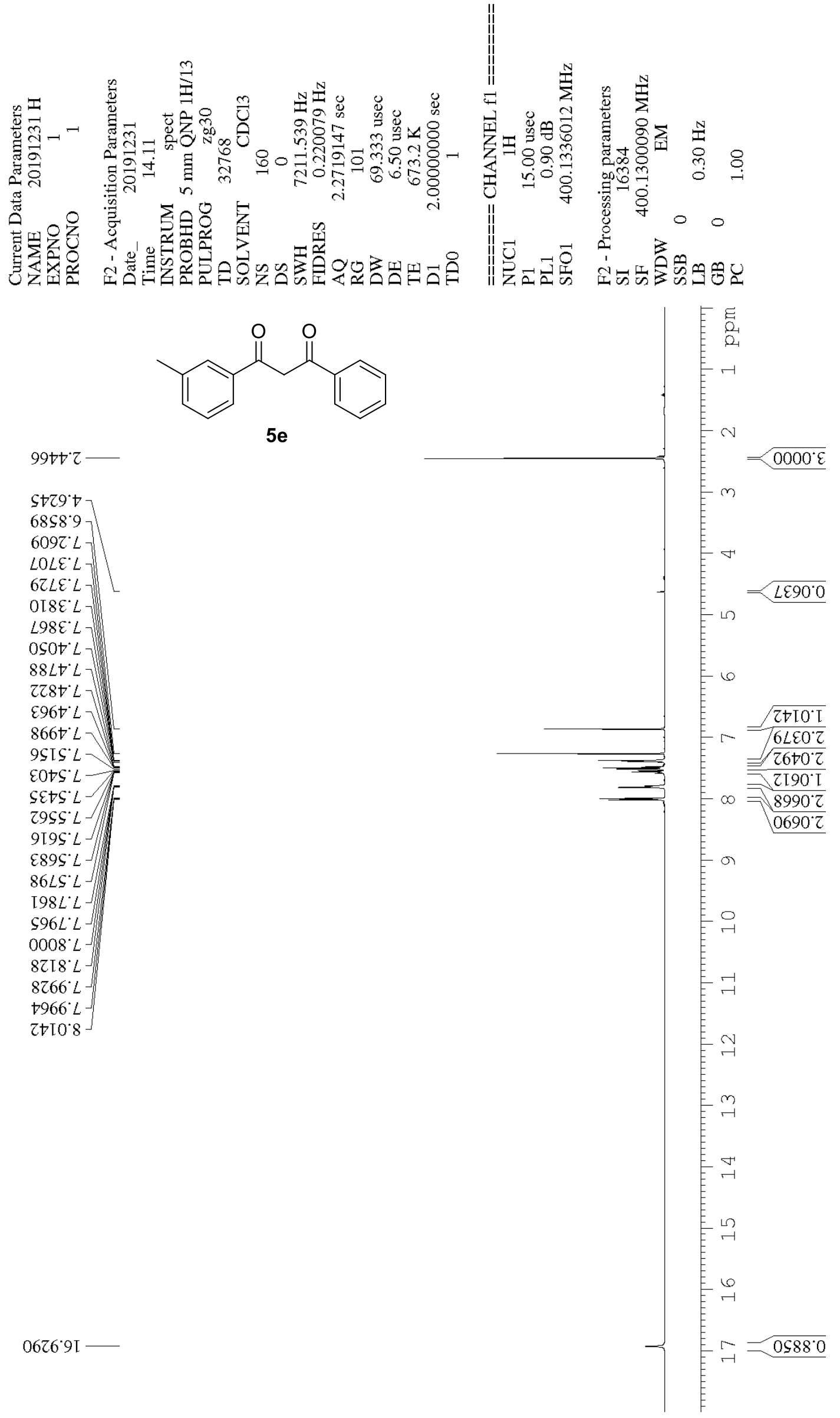




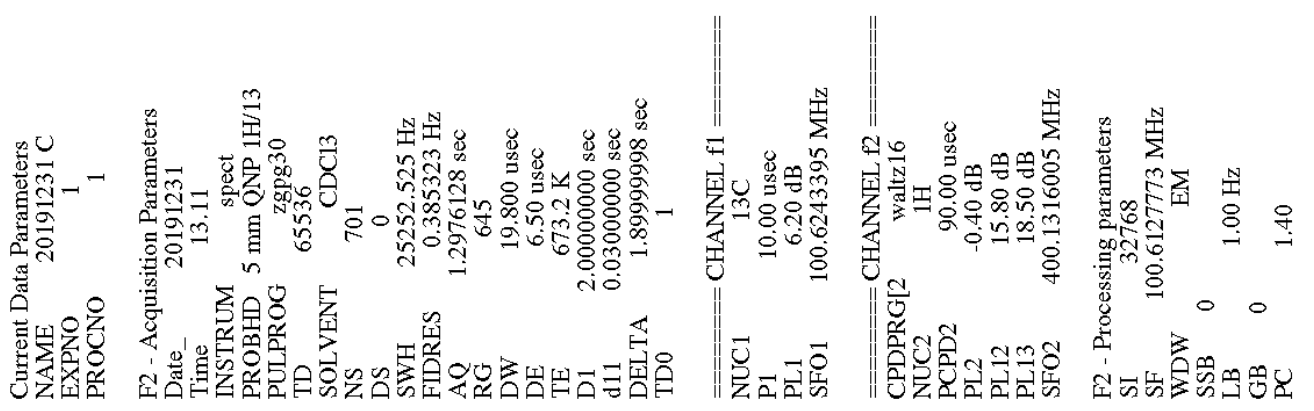

$\varepsilon \subseteq 8 \mathcal{E}^{\prime} \mathrm{IZ}$<smiles>Cc1cccc(C(=O)CC(=O)c2ccccc2)c1</smiles>

$Z \angle 89^{\circ} 9 L$

$2+00 . \angle L$

$\checkmark \angle O Z ' L L$

SZZELL

$80 t T^{\circ} \mathcal{E} 6$

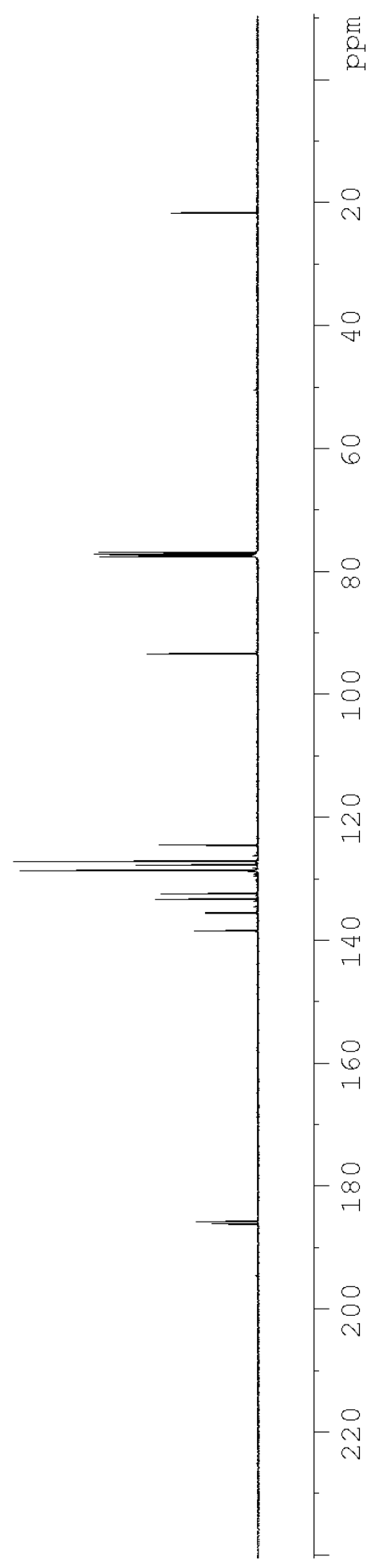

$\varepsilon 69 \varsigma^{\circ} \$ 8 \mathrm{I}-$

ZL86. $\mathrm{S} 8 \mathrm{I}$

OII I $\angle Z \mathrm{I}$

$6 \angle L 9 \cdot \angle Z I$

DIZS'8ZI

$9129^{\circ} 8 Z 1$

SIt $Z \mathcal{E} \in \mathrm{EI}$

LStT'SEI

I90S'SEI

ऽऽZt' $8 \varepsilon \mathrm{I}$

$5 e$

? 

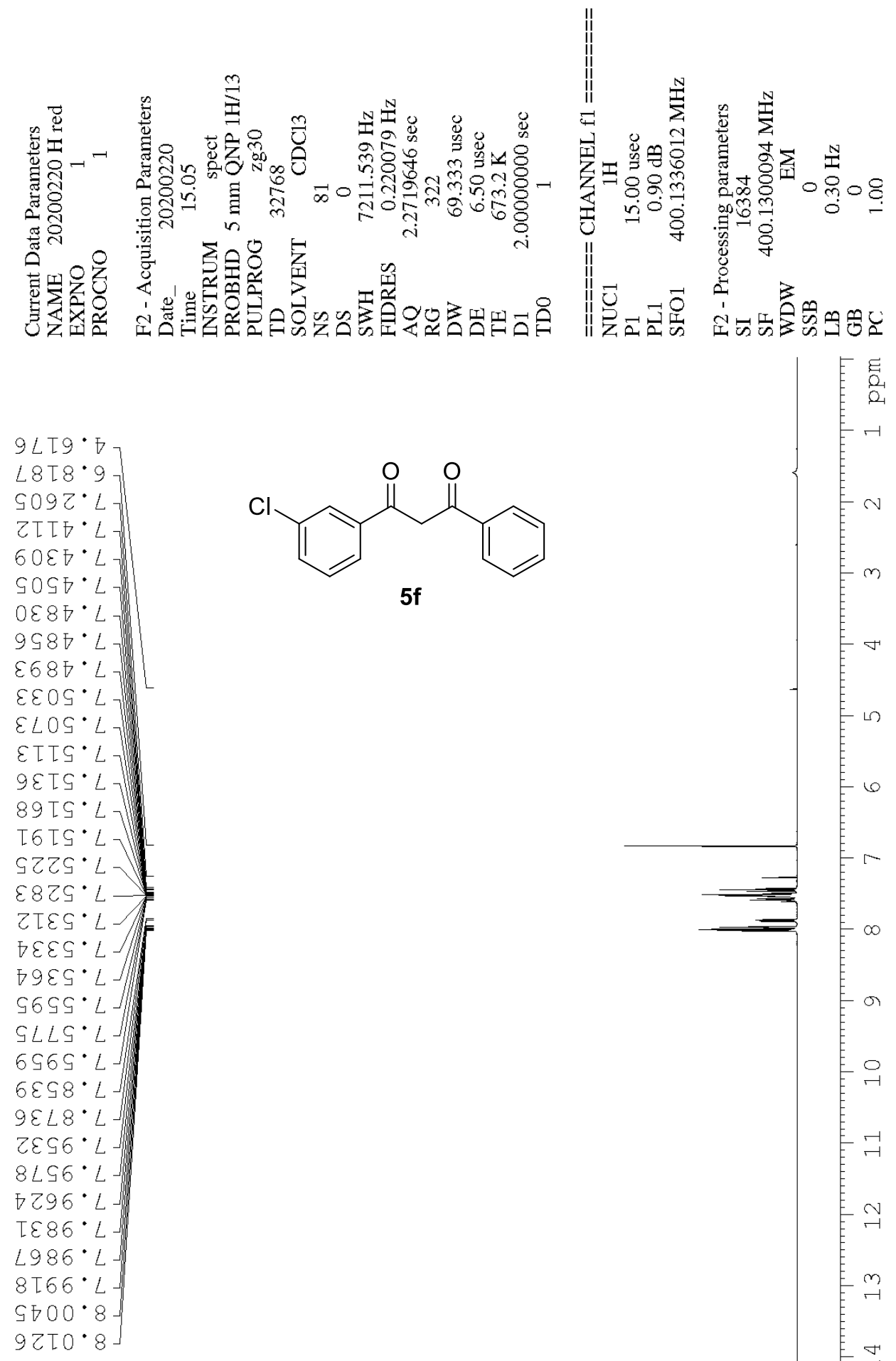<smiles>O=C(CC(=O)c1cccc(Cl)c1)c1ccccc1</smiles>

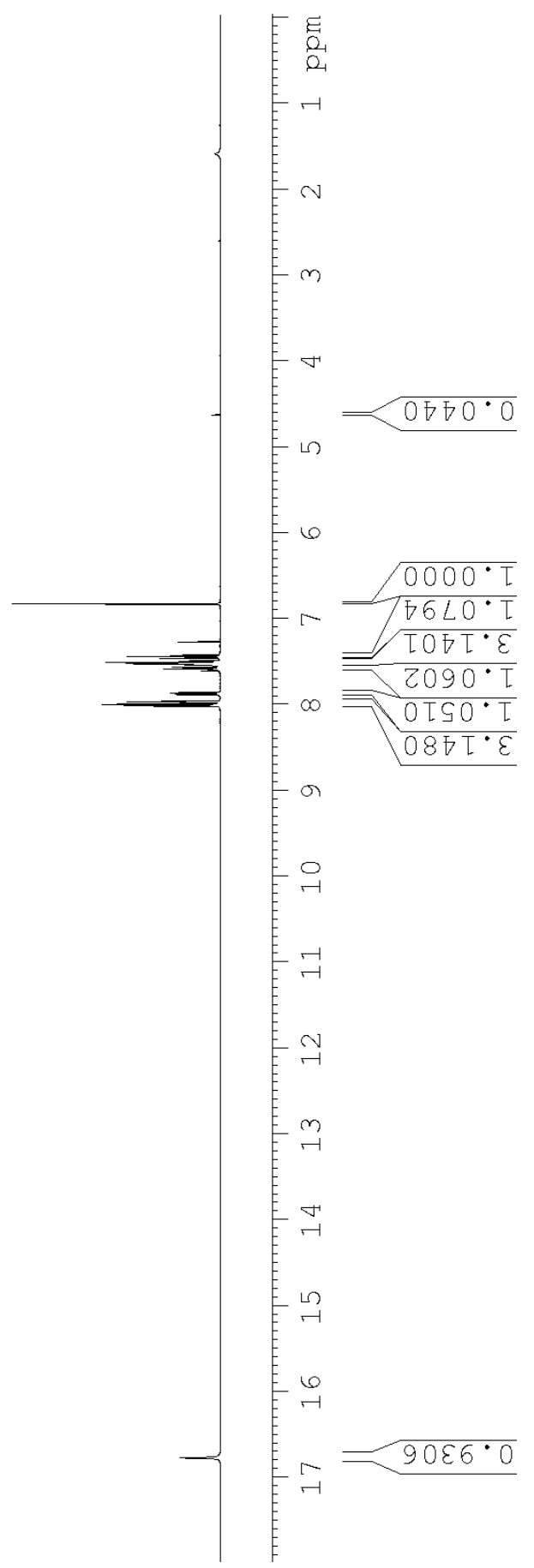



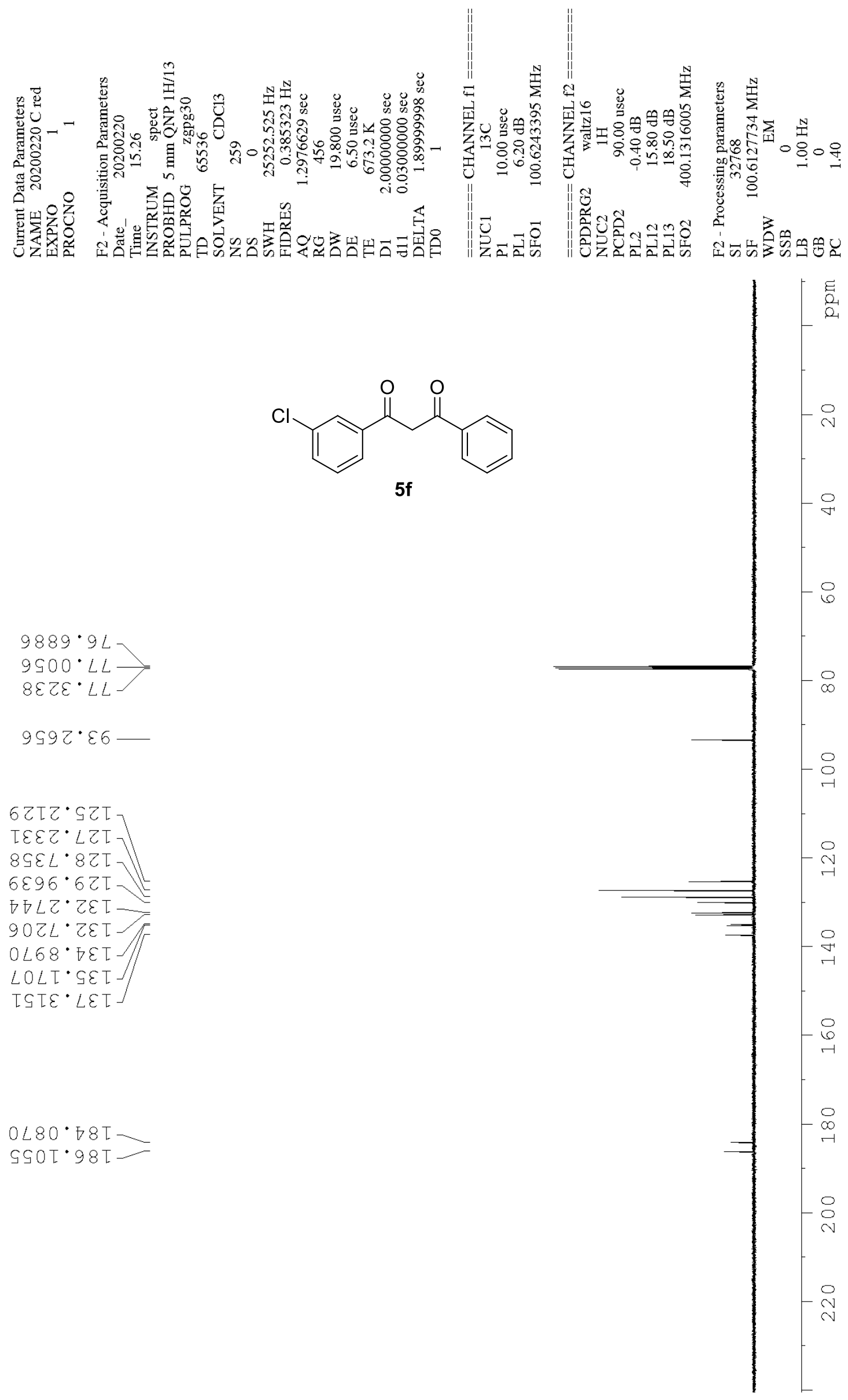

$0 \angle 80^{\circ} 78 T$

G90I.98I 

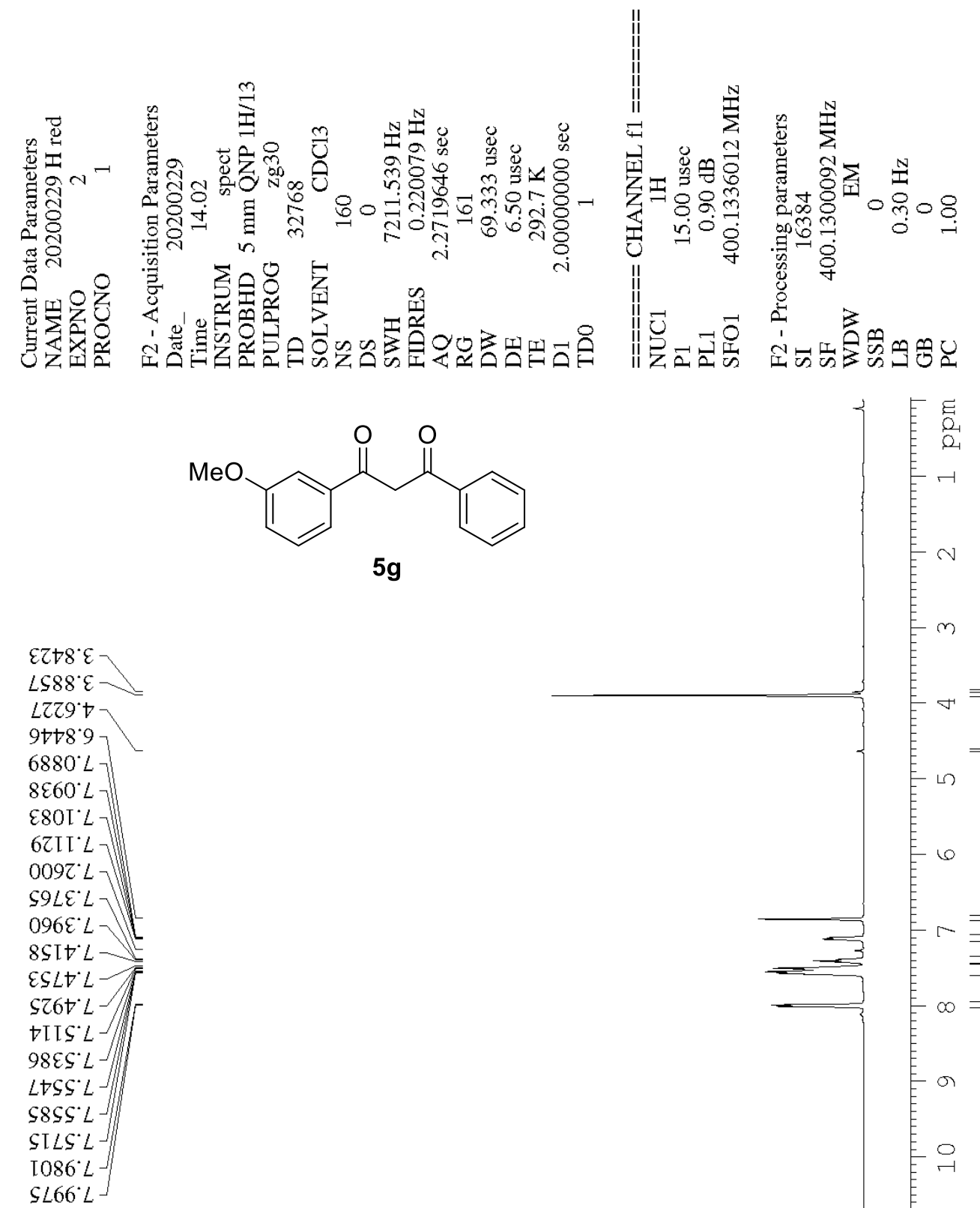<smiles>COc1cccc(C(=O)CC(=O)c2ccccc2)c1</smiles>

$5 g$

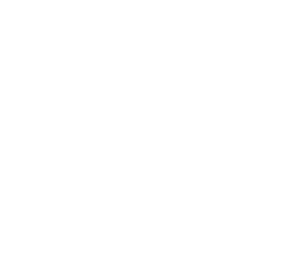




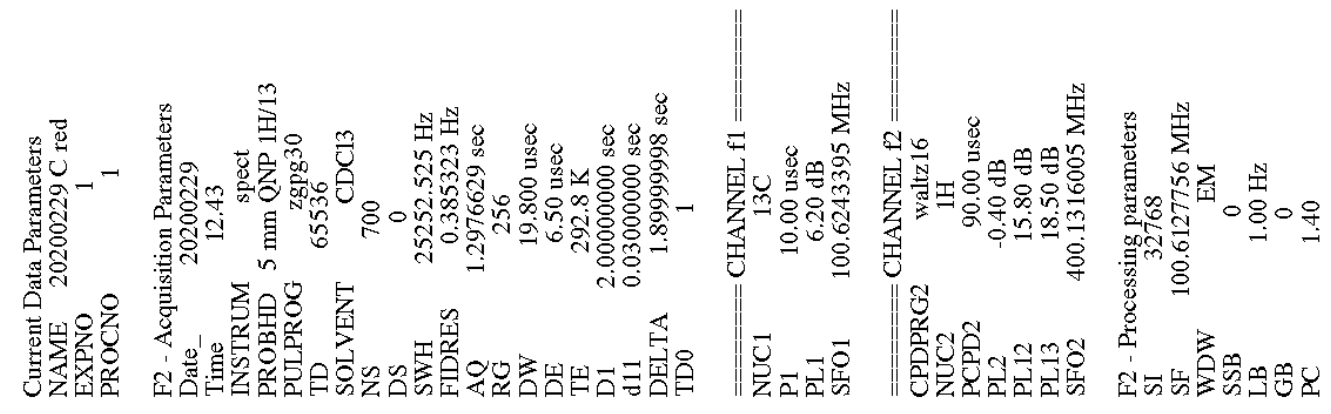<smiles>COc1cccc(C(=O)CC(=O)c2ccccc2)c1</smiles>

LZt'SS

$069^{\circ} 9 L$

$\angle 00^{\circ} \mathrm{LL}$

SZE $L L$

$\angle 6 Z^{\circ} \mathcal{E} 6$

IZ6.' I I -

885'8I I

I $L S 6$ I I

$8 \mathrm{II} \angle Z \mathrm{I}$

I99.8ZI

LZ9.6ZI -

ESt $\mathcal{E} \mathrm{I}$

$\triangleright \subseteq \mathcal{E} \subseteq \mathcal{I} \mathrm{I}$

$5 g$

$\angle 86^{\circ} 9 \varepsilon \mathrm{I}-$

$\downarrow \mathcal{H} 86 \varsigma \mathrm{I}$

$8 L \mathcal{E}^{\prime} \mathrm{S} 8 \mathrm{I}-$

$8 \mathcal{E} 8^{\circ} \mathrm{S} 8 \mathrm{I} \longrightarrow$

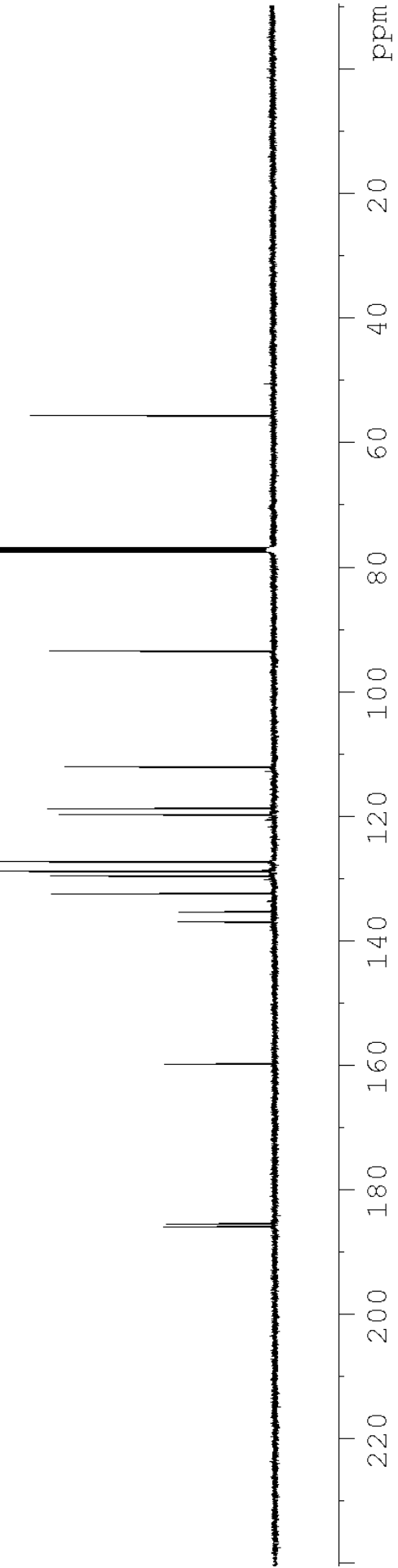



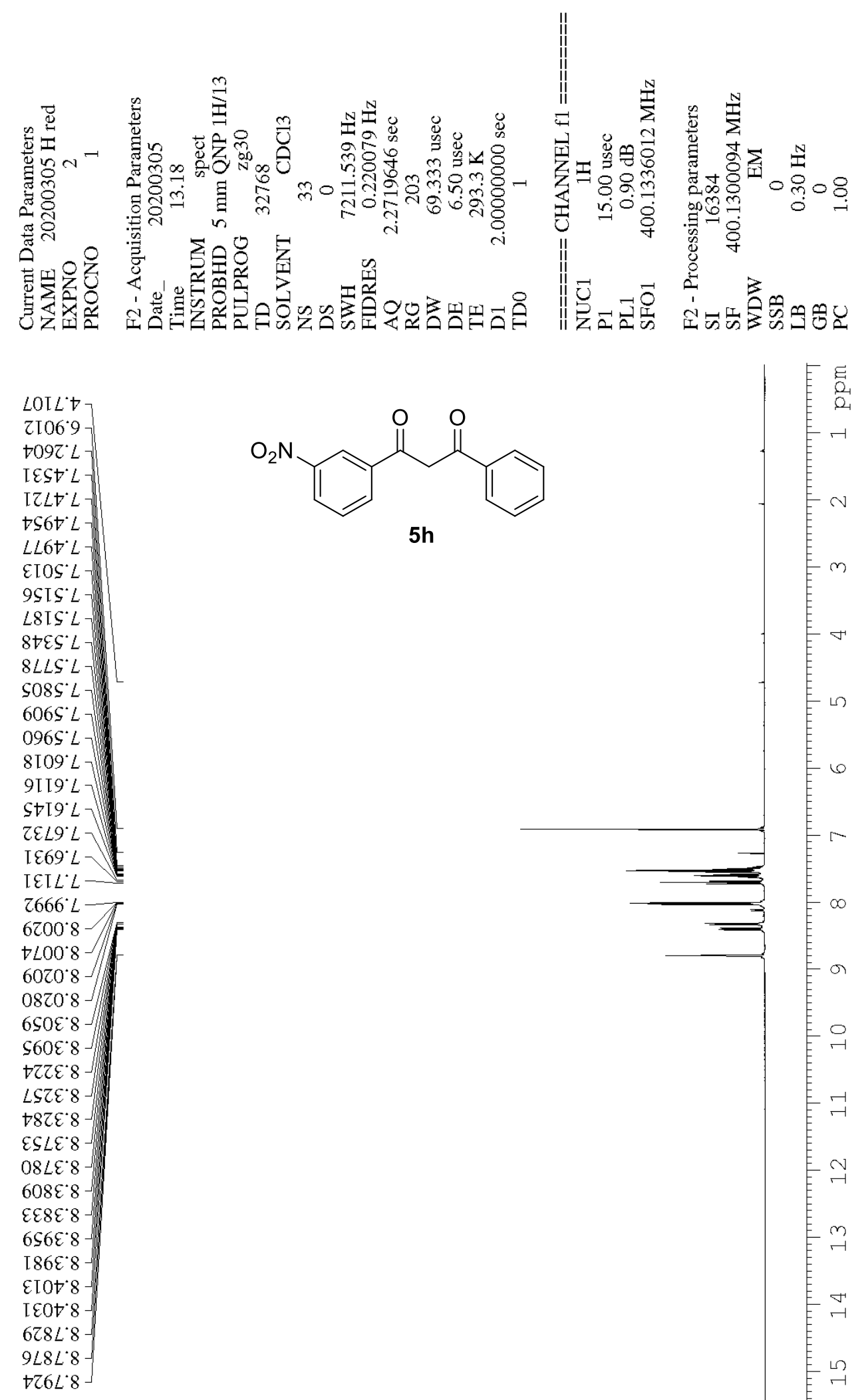<smiles>O=C(CC(=O)c1cccc([N+](=O)[O-])c1)c1ccccc1</smiles>

$606 S^{\circ} \mathrm{L}$

9I $19{ }^{\circ} \mathrm{L}$

IE69.

IEI $L L^{\circ} L$

$5 h$

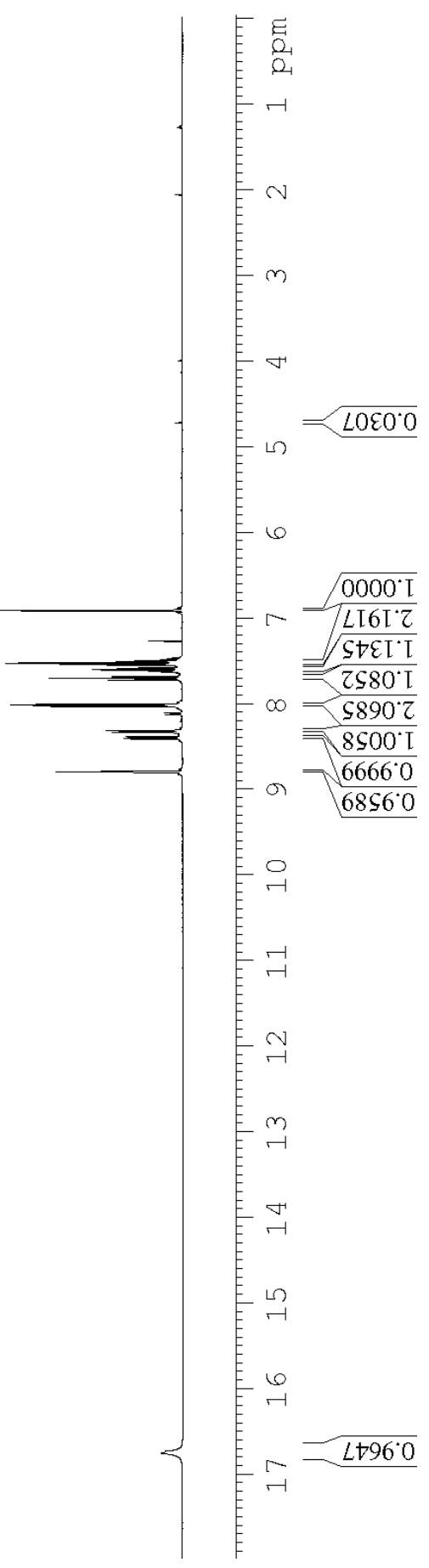

$\varsigma \mathcal{E}+L^{\circ} 9 \mathrm{I}$ 


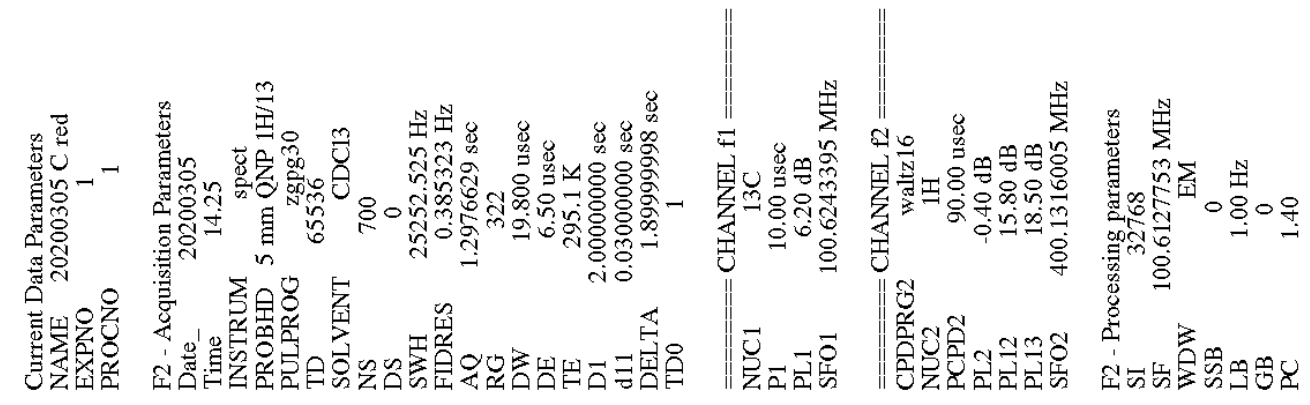

¡SSE $\mathcal{E}^{\circ} 6$

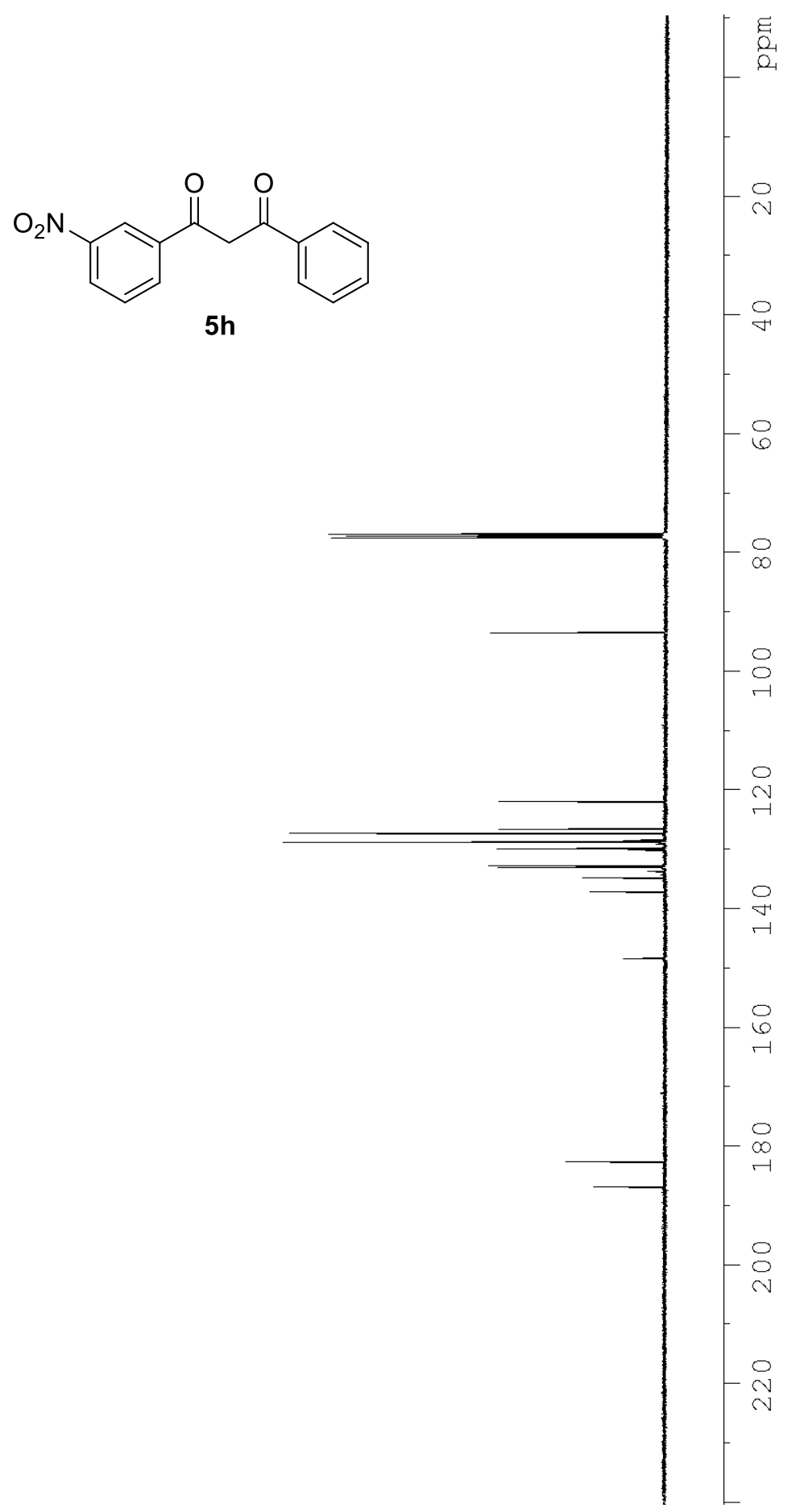

$5 \mathrm{~h}$

LIL6'IZI IZ9S.9ZI $\angle L Z E L Z \mathrm{I}$

$\varepsilon 86 L$ '8ZI

6898.6ZI -

ZOSLZEI -

$\varsigma$ † $0^{\circ} \mathcal{E} \mathrm{I}$

$0 \mathcal{C} 08^{\circ} \sqcup \varepsilon \mathrm{I}$

$\varepsilon 8 L I ' L E I$

ESIt $8+1-$

E6ZS'Z8I

E80 L' $98 \mathrm{I}$ 


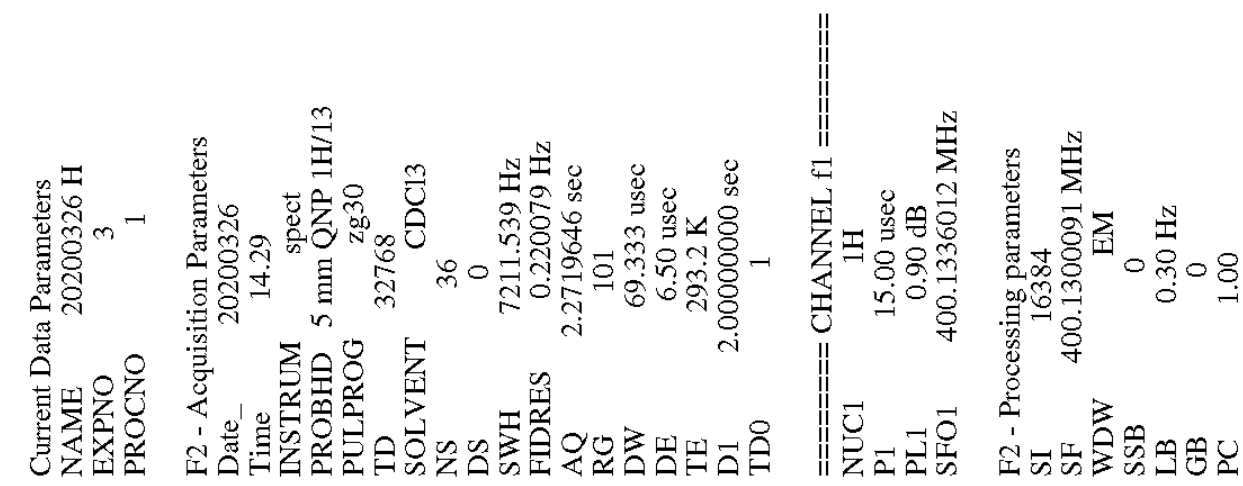

$82 \angle 9^{\circ}$

$08 โ 6 \cdot 9$

L092 ${ }^{\circ} L$

$766 \varepsilon^{\circ} L$

$L L T \sigma^{\circ} L J$

ogE⿰ ${ }^{\circ} L$

$8 \varepsilon \angle \nabla^{\circ} \angle$

งEธ $\sigma^{\circ}$

OrTS.

9 TES

万6S $9^{\circ}$

$\nabla L L S^{\circ}$

$\angle S 69^{\circ} L-$

$\nabla 0 L 9^{\circ} / \mathrm{L}$

$L \varepsilon L 9^{\circ} L J$

$\nabla G[L \cdot L]$

$\varepsilon 9 \varepsilon L^{*} L$

$L \varepsilon[0 \cdot 8-$

$\varepsilon L T O \cdot 8-$

ZSEO 8 -

$90 \angle 0 \cdot 8$

$9[60 \cdot 8]$<smiles>O=C(CC(=O)c1ccc(-c2ccccc2)cc1)c1ccccc1</smiles>

$5 \mathbf{i}$ 


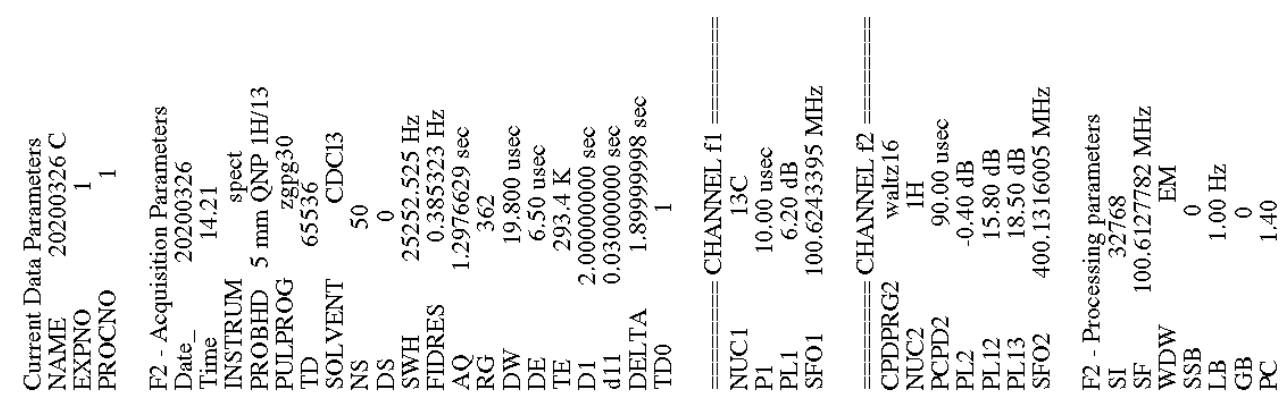

$0 \angle 89^{\circ} 9 L$

$\mathcal{E}+00^{\circ} L L$<smiles>O=C(CC(=O)c1ccc(-c2ccccc2)cc1)c1ccccc1</smiles>

$\mathrm{Ph}_{\mathbf{i}}$

$\downarrow T \angle 0^{\circ} \mathcal{E} 6$

†8EI $\angle Z$

$99 \angle \mathrm{I} \cdot L Z \mathrm{I}$

ZE9Z: $\angle Z I$

$96 \angle 9^{\circ} \mathrm{LZI}$

$6 \triangleright \varepsilon I$ ' $8 Z \mathrm{I}$

IE\$9'8ZI

6LI6.8ZI

E9Z† ZEI

6S9I' $\mathrm{EI}$

Эtos'ce

I $\angle+8^{\circ} 6 \varepsilon \mathrm{I}$

$\varepsilon Z t I \cdot S t I-$

$99 \mathrm{IZ} \$ 8 \mathrm{I}-$

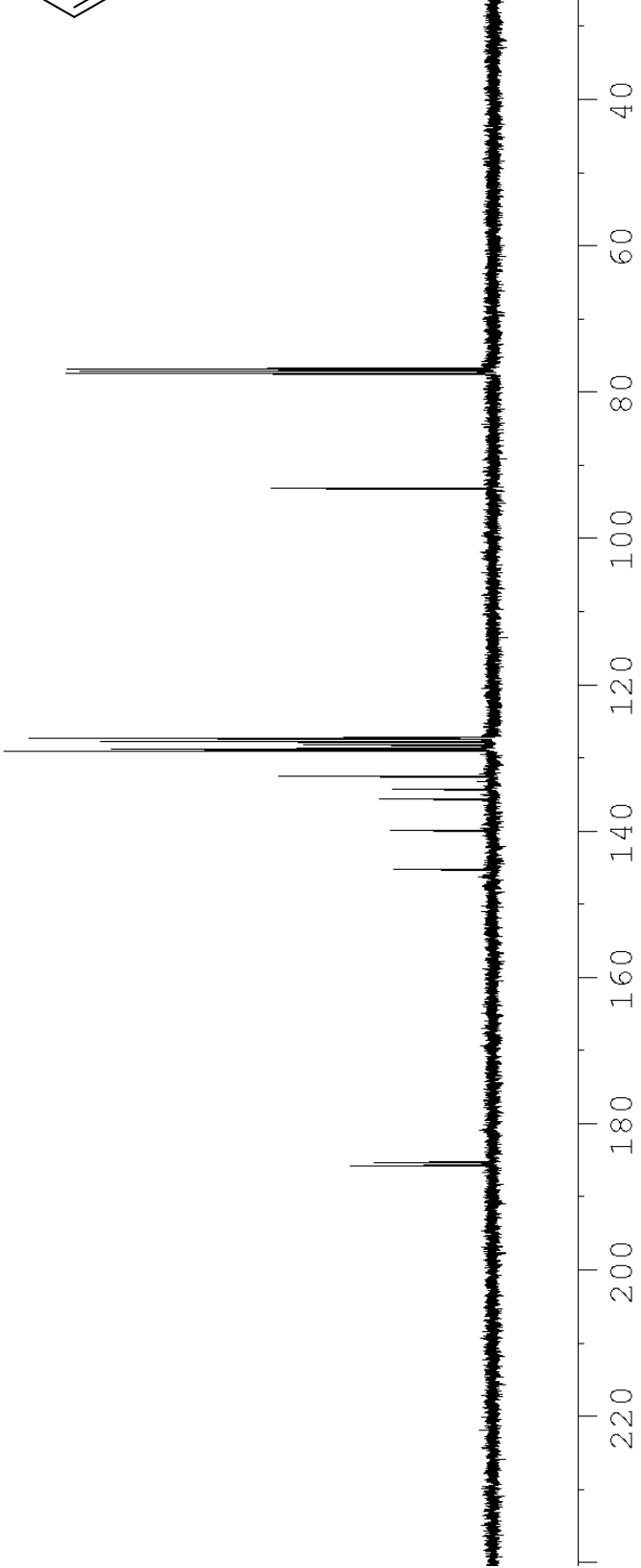




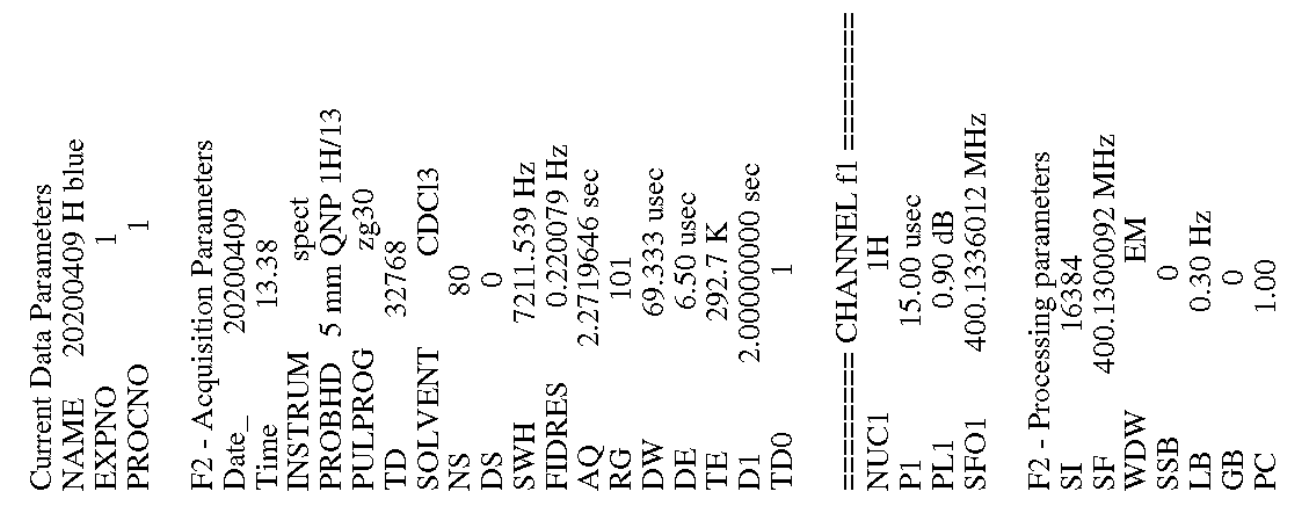<smiles>Cc1ccc(C(=O)CC(=O)c2ccccc2)cc1</smiles>

5j
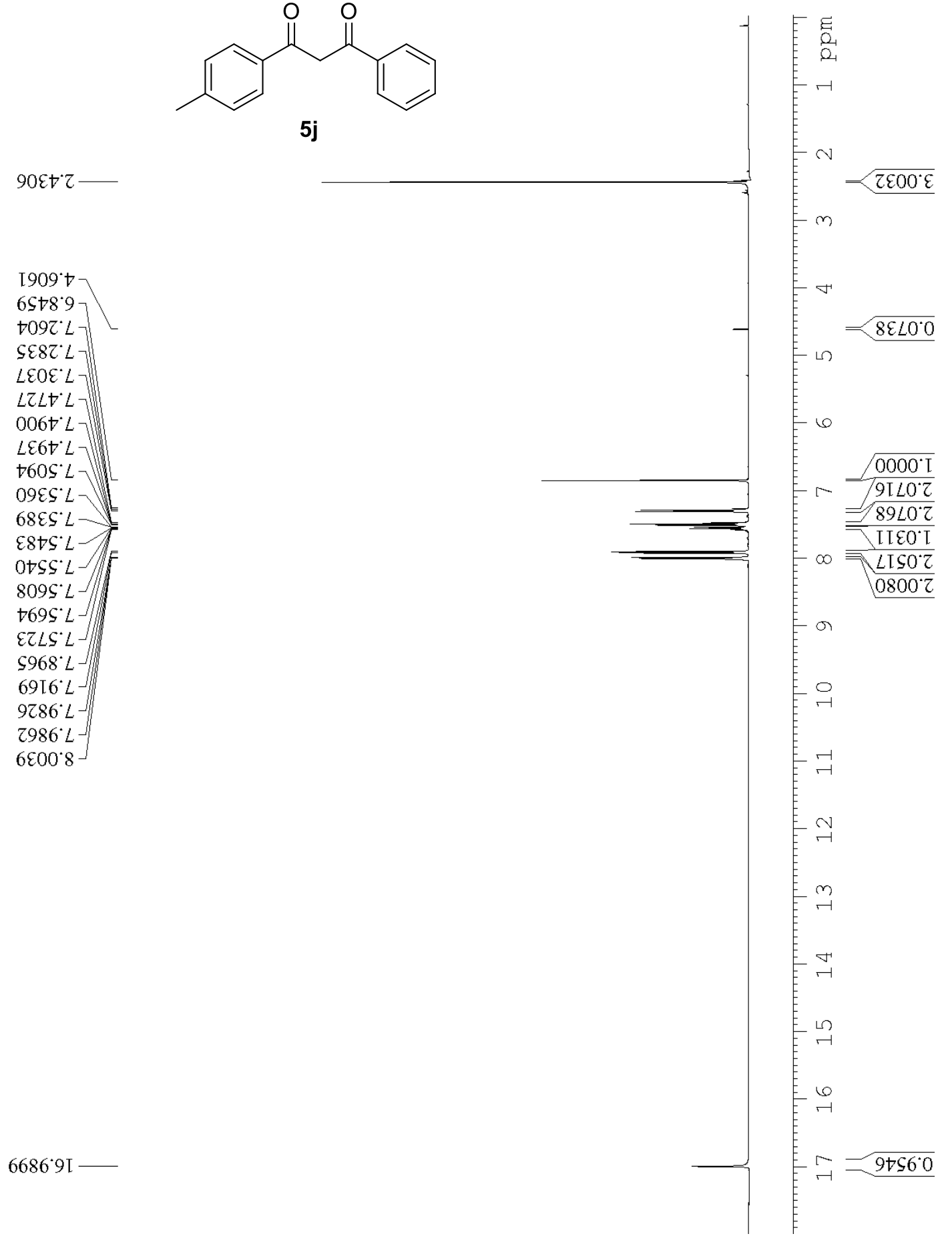


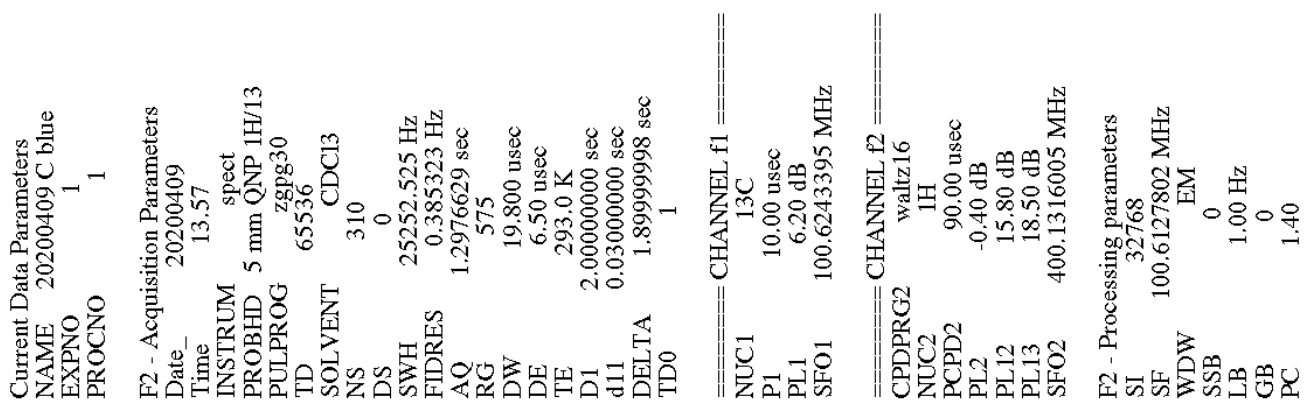

088ऽ $I Z$<smiles>Cc1ccc(C(=O)CC(=O)c2ccccc2)cc1</smiles>

5j

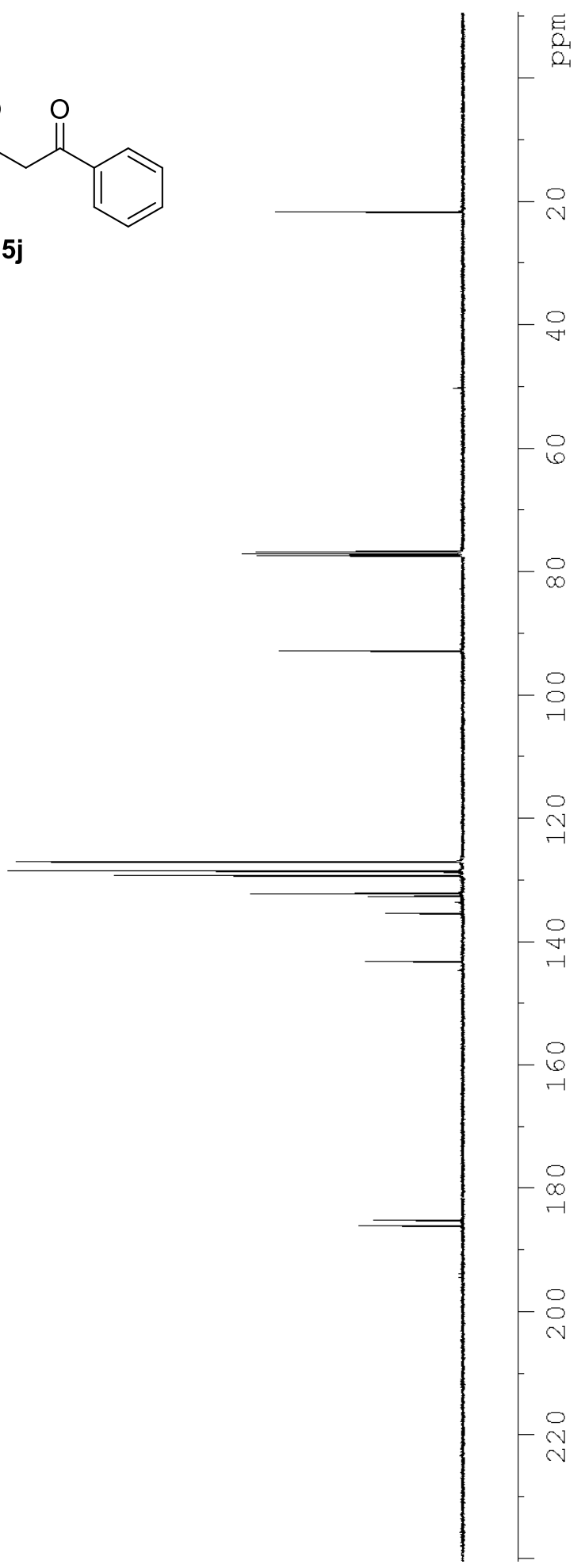

$2989^{\circ} 9 L$

$0+00 . \mathrm{LL}$

8IZE $L L$

$6+\mathcal{E} L \cdot 26$

$\angle 820^{\circ} \angle Z \mathrm{I}$

$689 \mathrm{I}^{\circ} \mathrm{LZI}$

6E8S'8ZI

$0 \angle t \varepsilon \cdot 6 Z I$

OI9Z'ZEI

ZLZL'ZEI

$6080^{\circ} \subseteq \mathcal{}$ I

E

0 


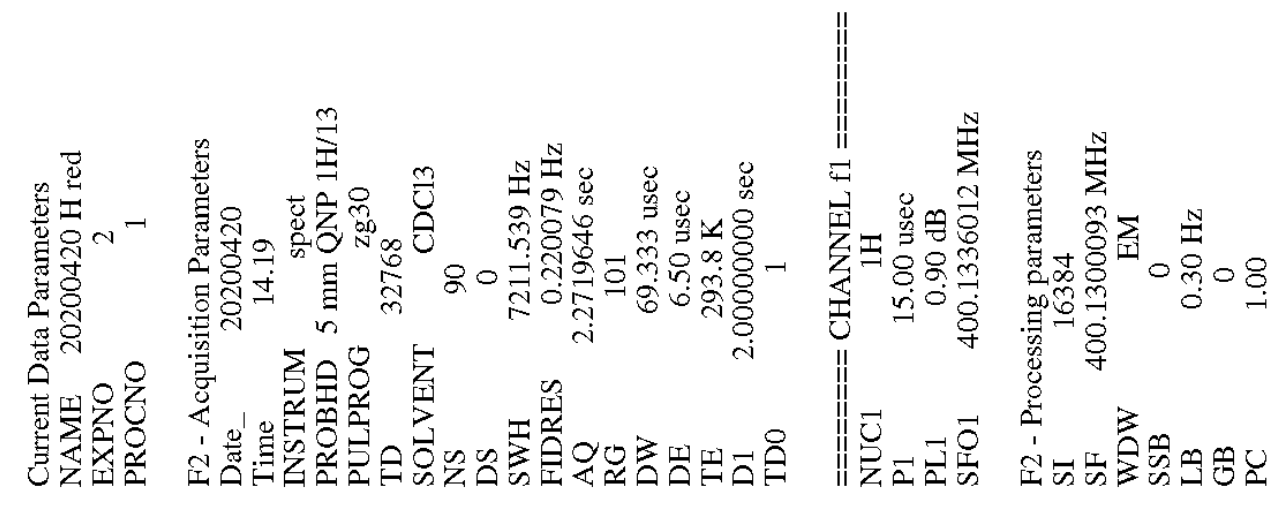<smiles>O=C(CC(=O)c1ccc(Cl)cc1)c1ccccc1</smiles>
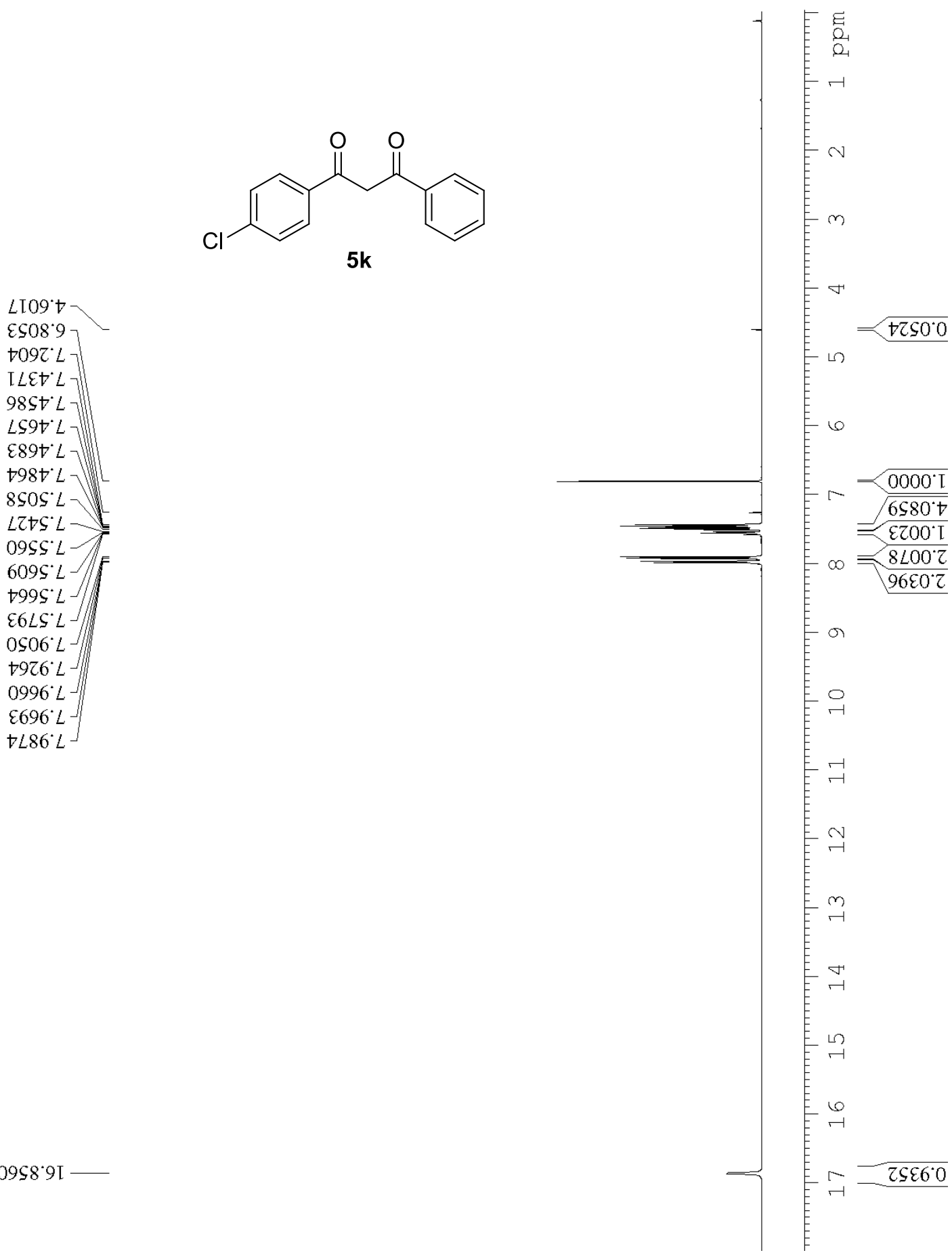


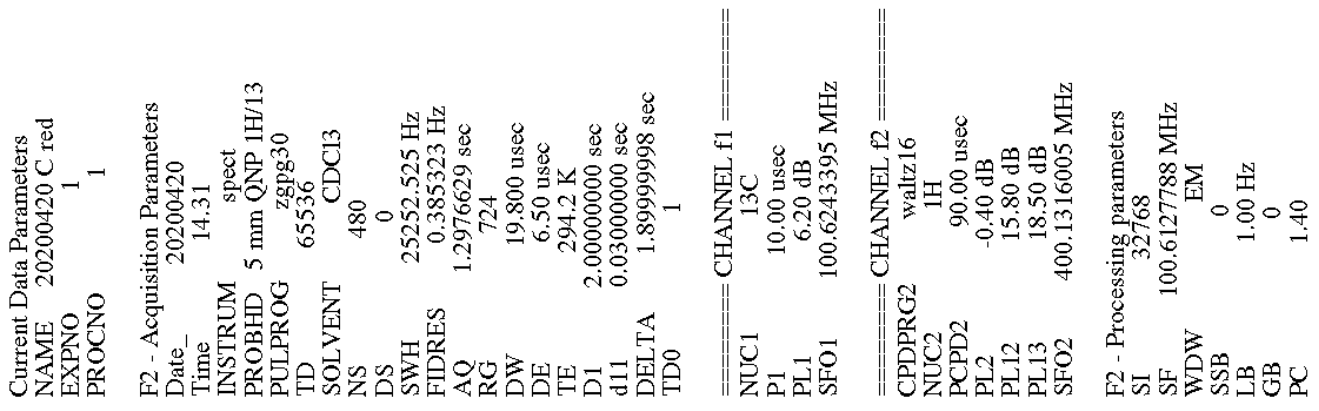

$9289^{\circ} 9 L$

$8000^{\circ} \mathrm{LL}$

I8I $\mathcal{L} L L$<smiles>O=C(CC(=O)c1ccc(Cl)cc1)c1ccccc1</smiles>

5k

$6816 \cdot 26$

I6I I' $\angle Z I$

29tt $8 \mathrm{ZI}$

$8959^{\circ} 8 \mathrm{ZI}$

9606 $8 \mathrm{ZI}$

$0 \angle 9 S^{\prime} Z E I$

$\varepsilon \sqcup \angle 8^{\circ} \varepsilon \mathcal{E} \mathrm{I}$

6LLI'SEI

IZ¥9.8\&I -

\$9S†' $78 \mathrm{I}-$

$88 \mathrm{I} L$ 'S $8 \mathrm{I}-$

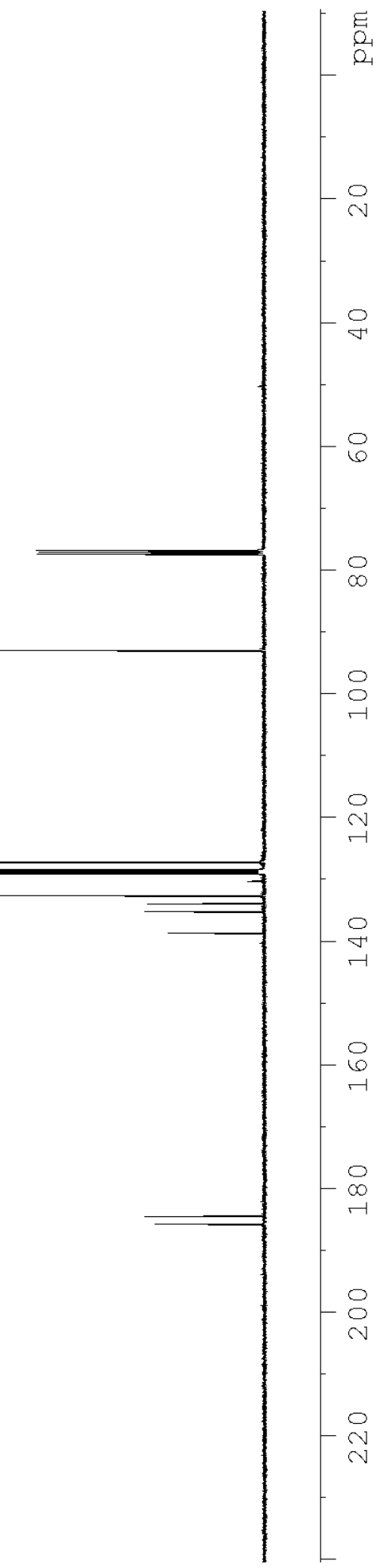



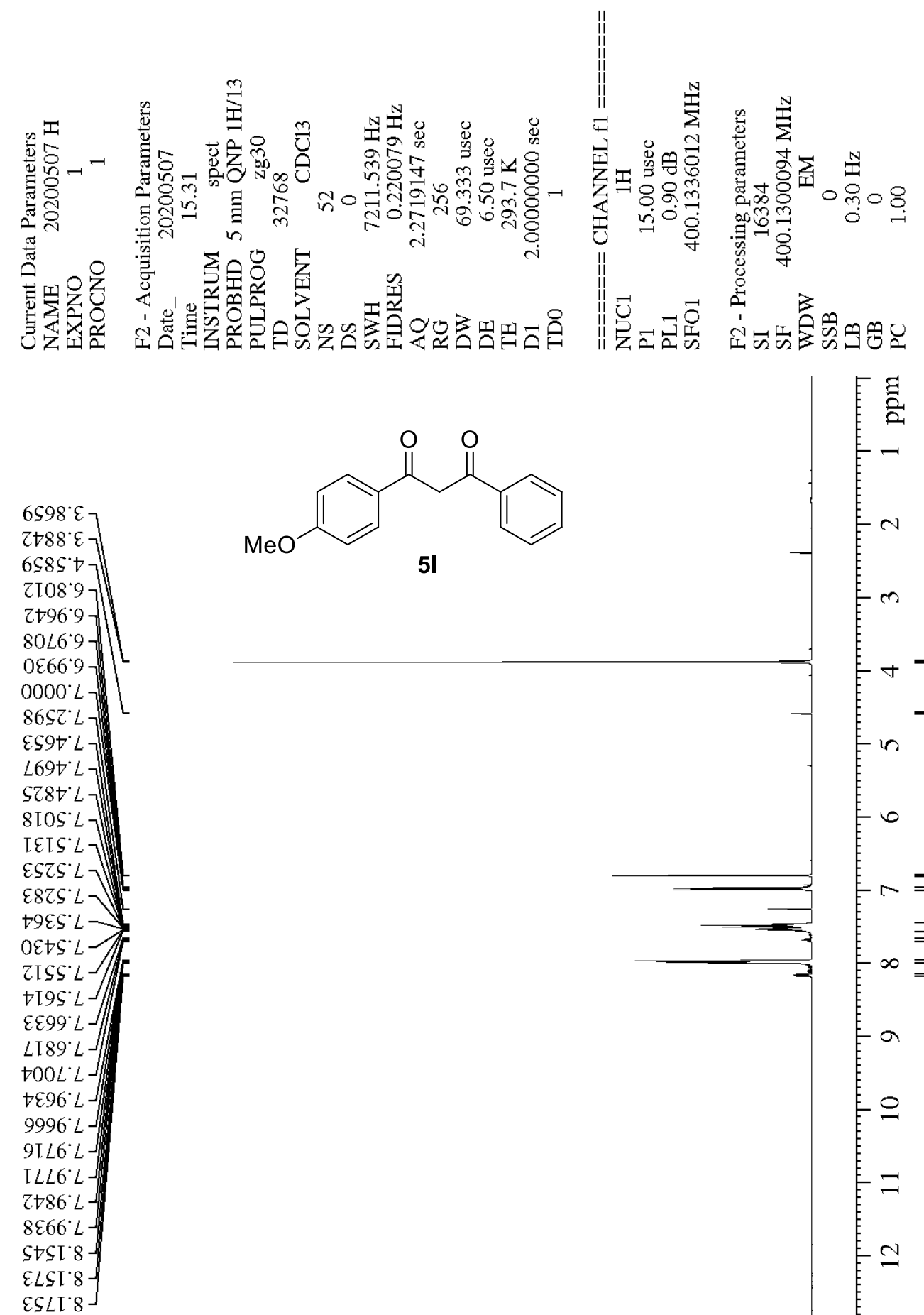<smiles>COc1ccc(C(=O)CC(=O)c2ccccc2)cc1</smiles>

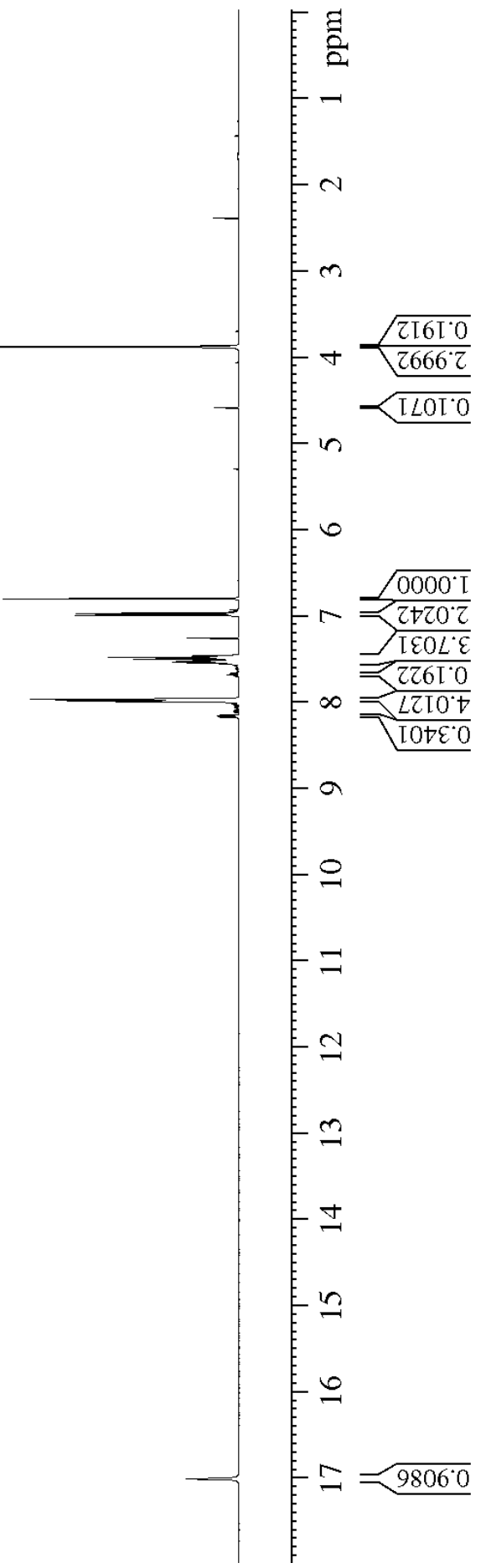




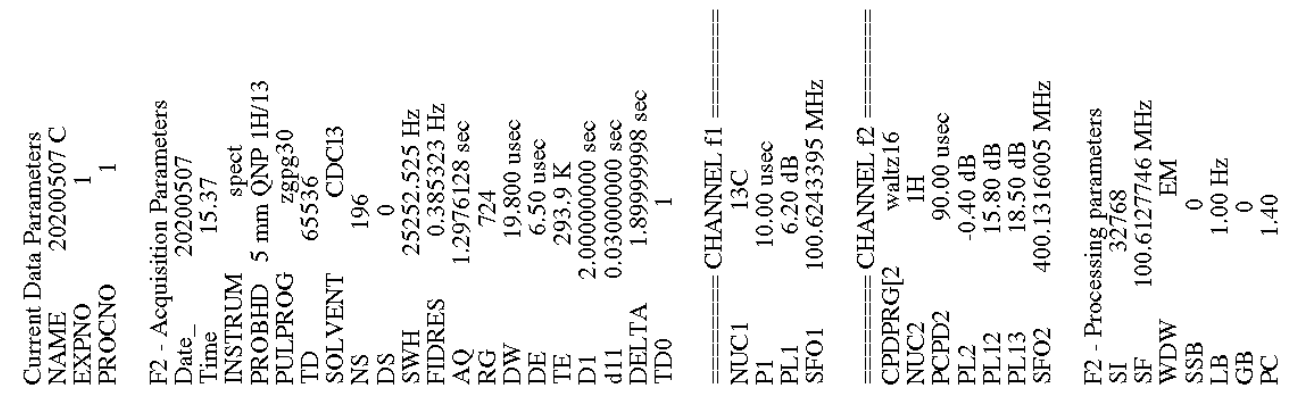<smiles>COc1ccc(C(=O)CC(=O)c2ccccc2)cc1</smiles>

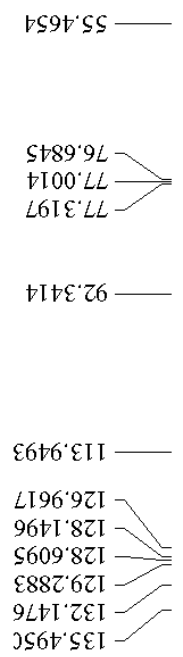

ILOZ'ย9I -

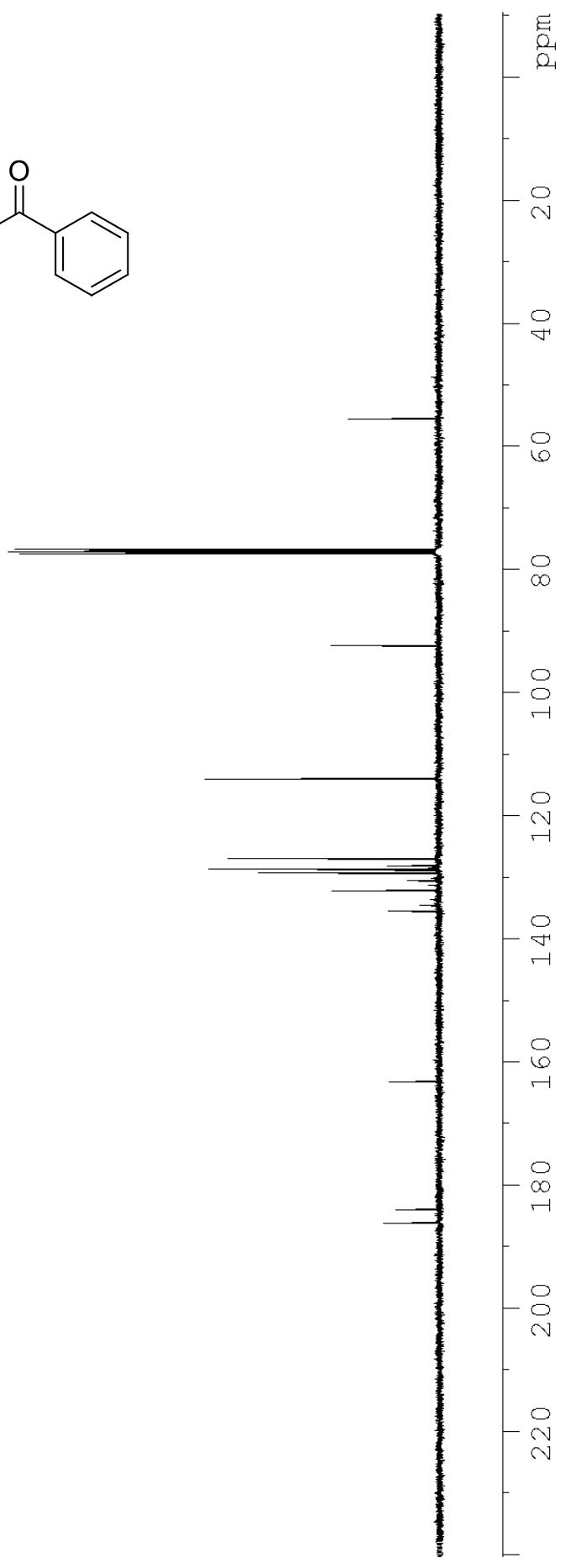

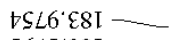
I6LI'981 - 

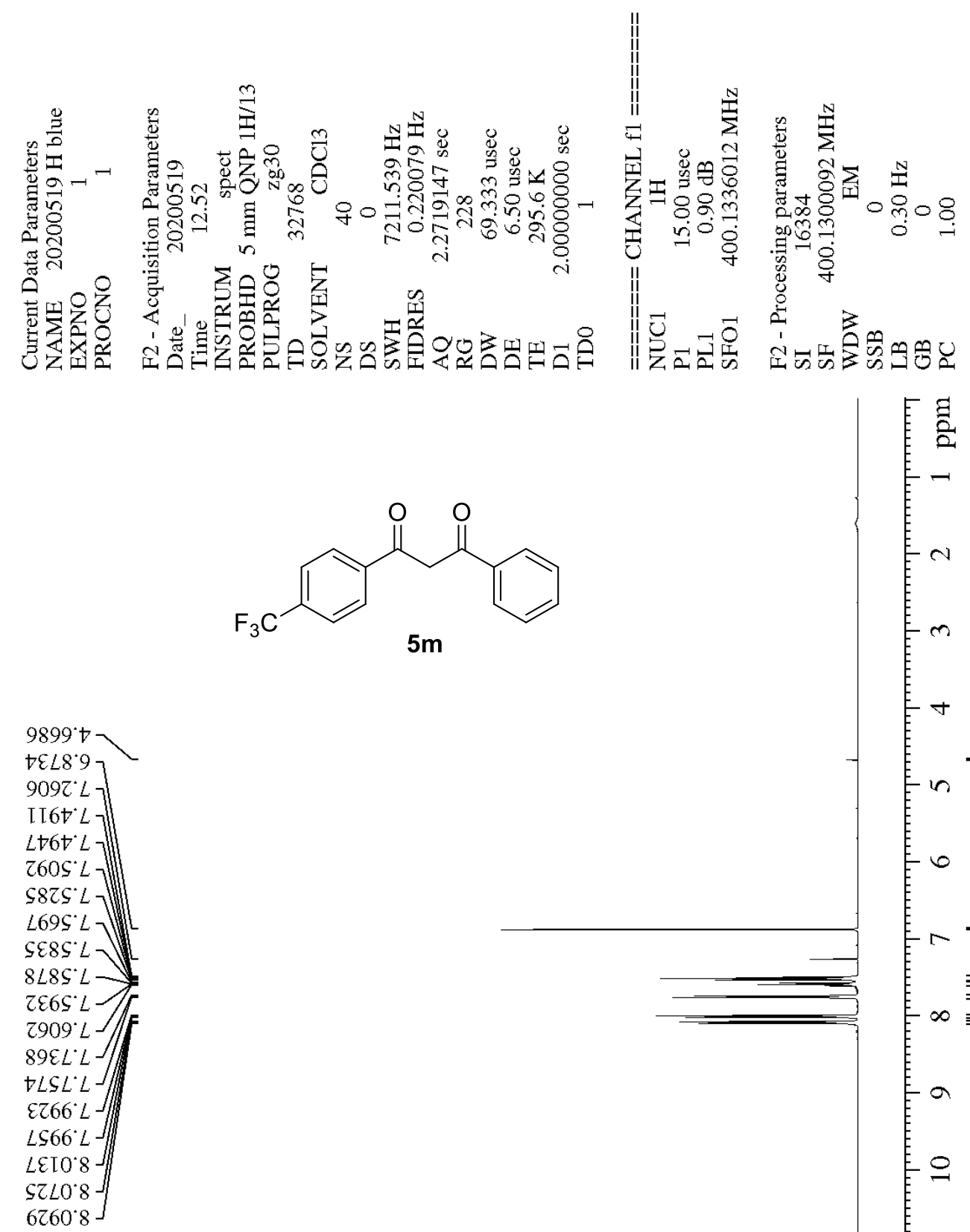<smiles>O=C(CC(=O)c1ccc(C(F)(F)F)cc1)c1ccccc1</smiles>

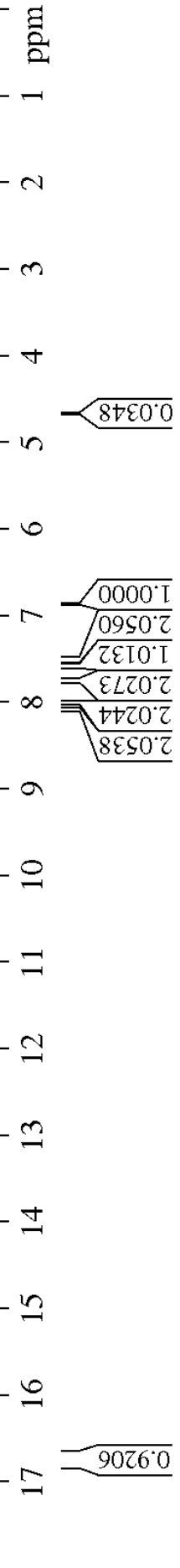




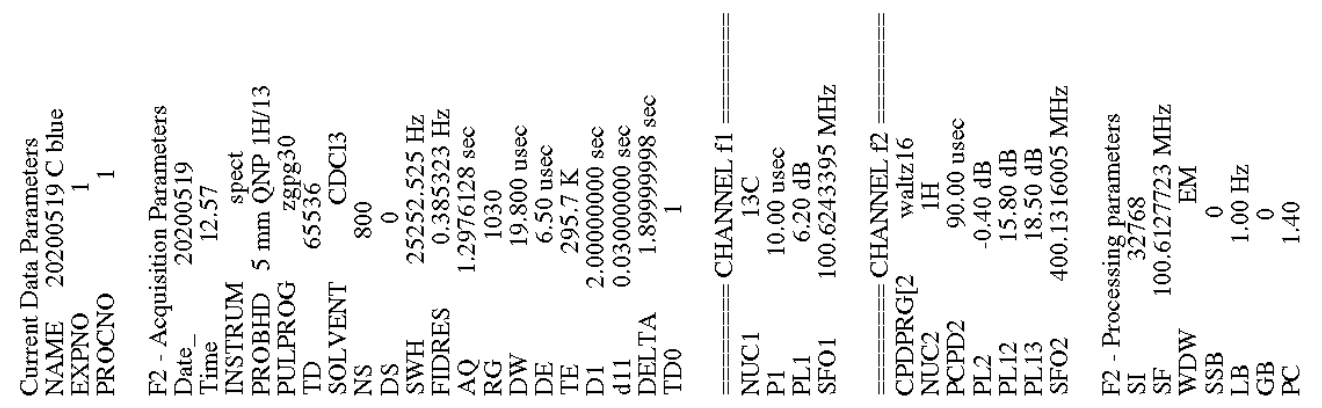

$8989^{\circ} 9 L$

$\angle E 00^{\circ} \mathrm{LL} \longrightarrow$

OZZE $L L$

$\angle Z \pitchfork 9^{\circ} \mathcal{E} 6$

LSIE'ZZI

$0+Z 00^{\circ} \subseteq Z I$

†96S'SZI

$3 \mathcal{E} 9^{\circ} \mathrm{C} I$

SI $\angle 9^{\circ}$ SZI

$980 L^{\circ} \mathrm{SZI}-$

ZZOE'LZI

69It' $\angle Z I$

Z89L'8ZI

$\mathcal{E} L 8^{\circ} Z \mathcal{} \mathrm{I}-$

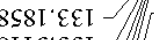

3IIS' $\mathcal{E}$ I

$\nabla 9 \mathcal{E} 8^{\circ} \mathcal{E}[-$

$0\left[9\left[{ }^{\circ} \downarrow \mathcal{E}\right.\right.$ I

99IZ' $\subseteq \mathcal{E L}$

$96 \$ 9^{\circ} 8 \varepsilon[$<smiles>O=C(CC(=O)c1ccc(C(F)(F)F)cc1)c1ccccc1</smiles>

$5 \mathrm{~m}$

Z0ZE' 8 I

E\$86.98I

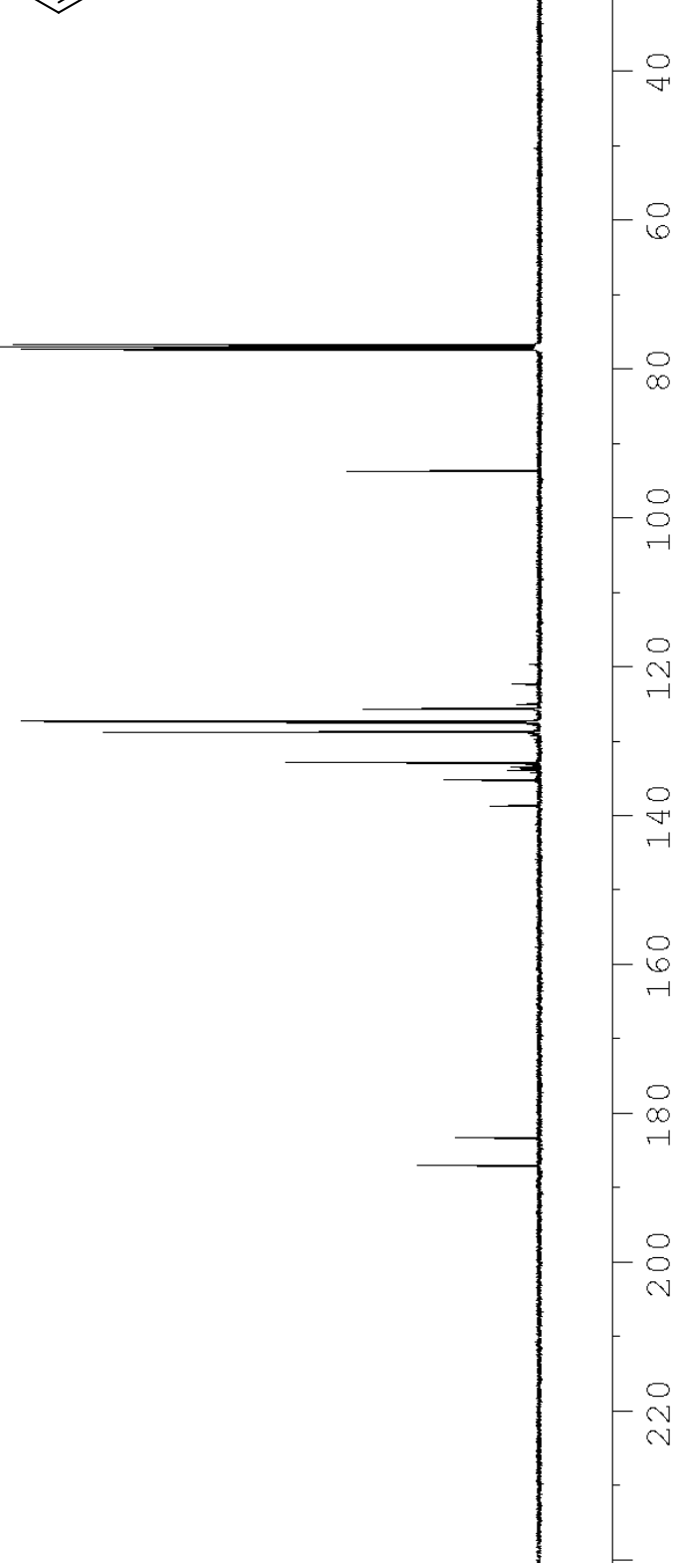




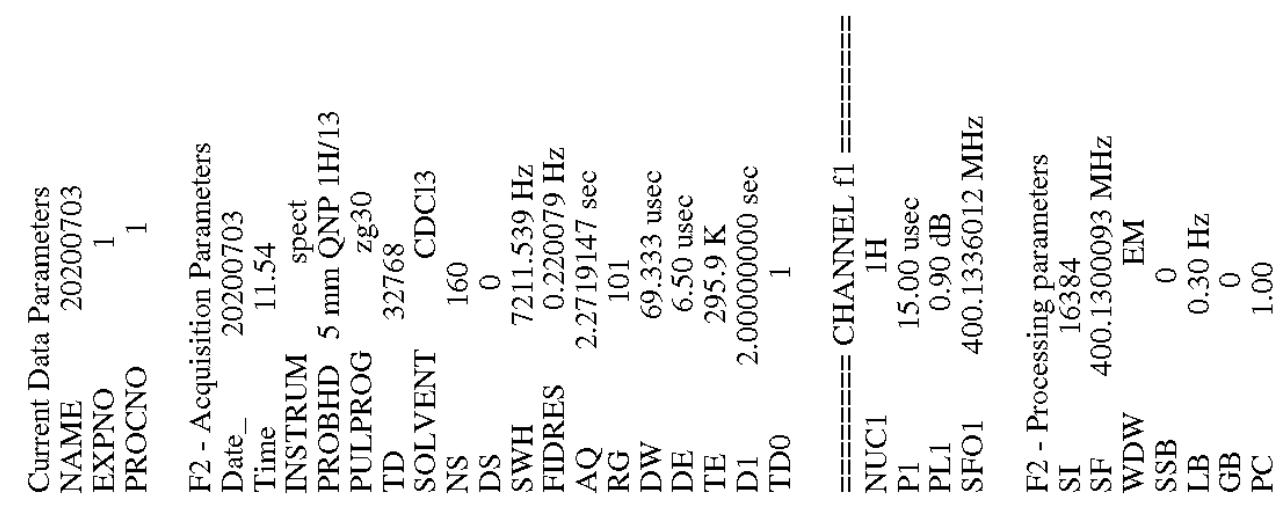

I $6 t s^{\circ} t$ $\nabla t+00^{\circ}$

I $\angle E L 9$ ]

$\angle T \angle 8^{\circ} 9$

$2768^{\circ} 9$

$0092^{\circ} L$

OLST $L$

OL9t $L]$

$\varepsilon Z \angle t^{\circ} L$

9[6t $L]$

$\checkmark 8 \mathrm{IS}^{\circ} \mathrm{L}$

$90 \varepsilon S^{\circ} L$

†OES $L$

$\varepsilon \varepsilon+S L$

$9+S S L$

26LS L

IE8 $S^{\circ} L$

$\angle 66 S^{\circ} L$

$\angle E 09^{\circ} L$

$0+t 6: L$

$0 \angle t 6 \angle \mathrm{L}$

$0 S 96^{\circ} \mathrm{L}$<smiles>O=C(CC(=O)c1ccc2c(c1)OCO2)c1ccccc1</smiles>

$5 n$

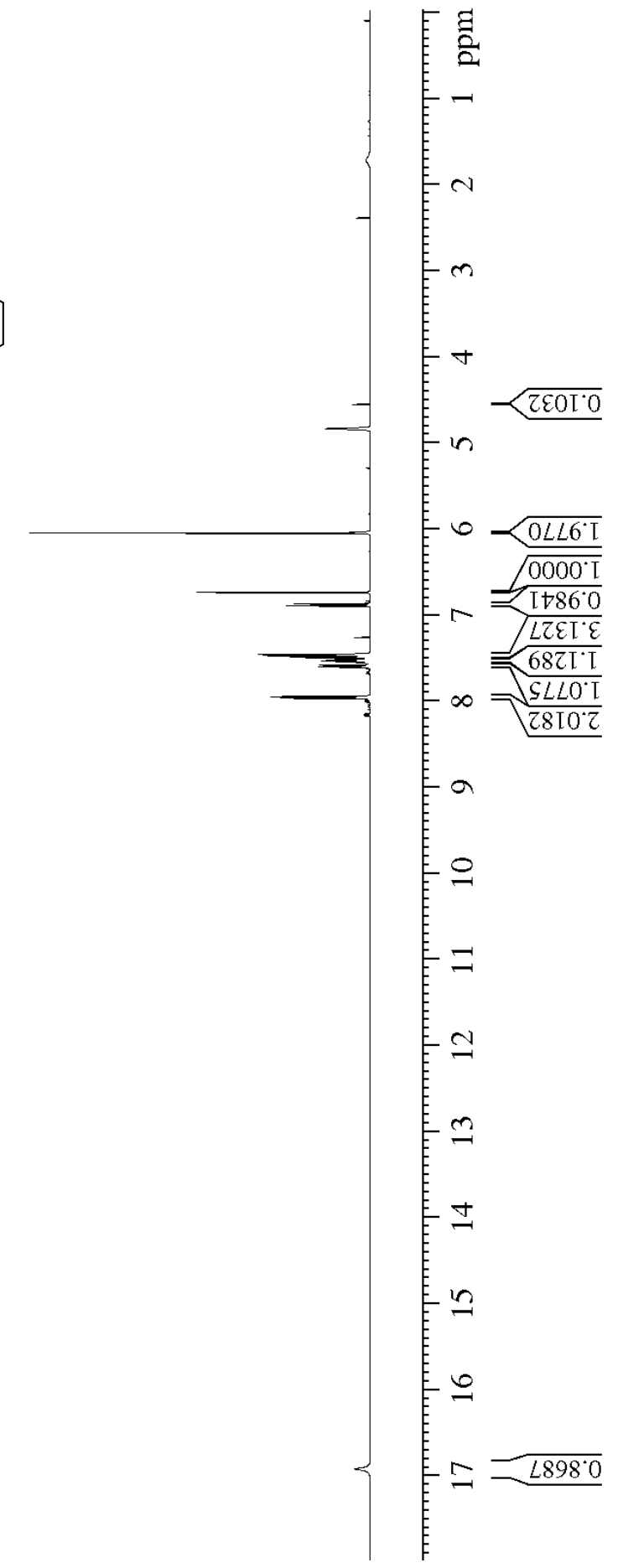




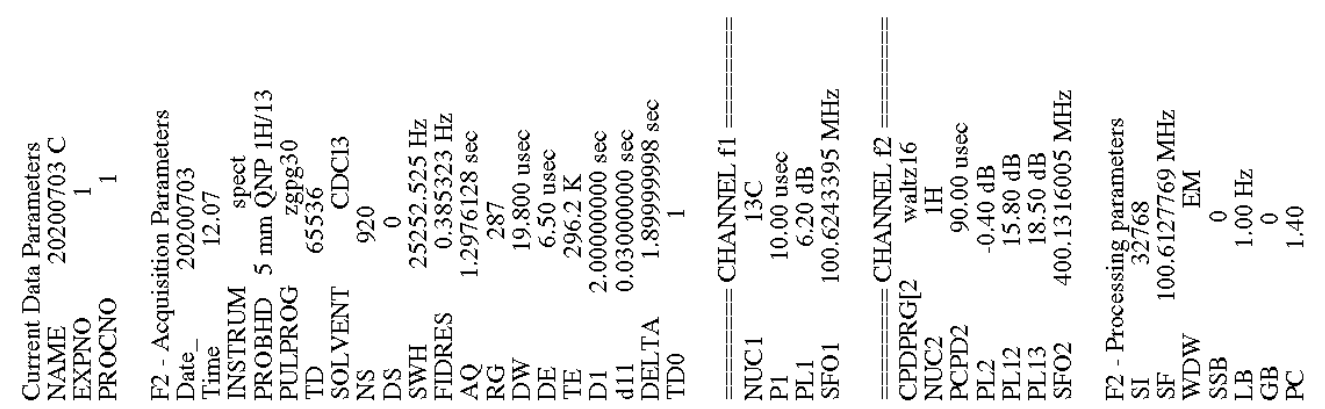<smiles>O=C(CC(=O)c1ccc2c(c1)OCO2)c1ccccc1</smiles>

$\angle 289^{\circ} 9 L$

$0000^{\circ} \mathrm{LL}$

E8IE'LL

06Lt'26

2928. I0I

EL8I' $L 0 \mathrm{I}$

$2 \downarrow L \mathrm{I} \cdot 80 \mathrm{I}-$

S\$Z6 ZZI

L8Z6.9ZI

IS6S'8ZI

6L90.0EI

6E6I' $Z \mathcal{~ I ~}$

Z8SZ ऽEI

669I' $8 \mathrm{tI}$

ZI8E'ISI —-

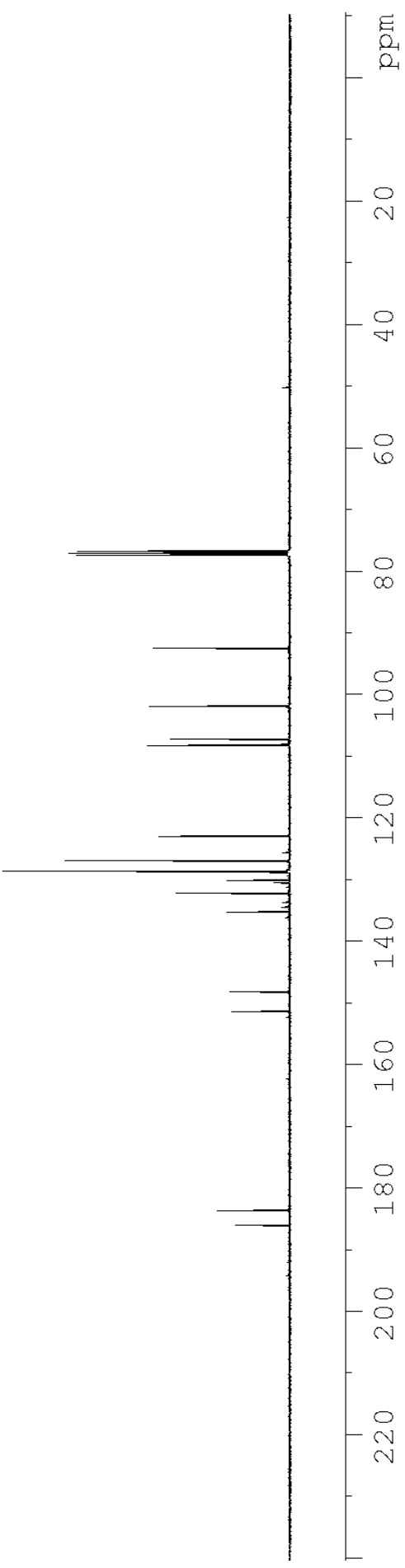

$88 \angle 9^{\circ} \mathcal{E} 8 \mathrm{I}$

L6ع0 $98 \mathrm{I}-$ 

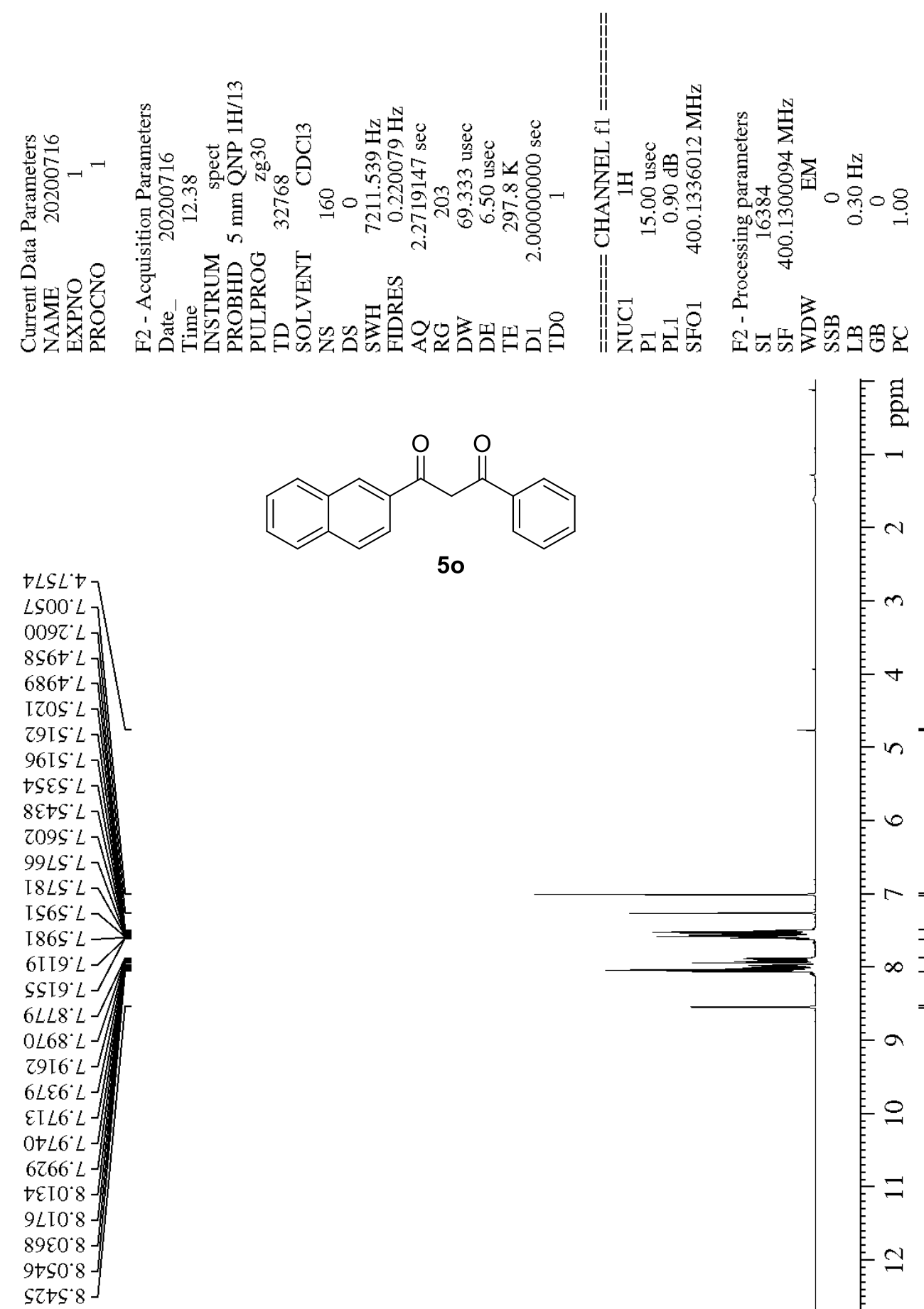<smiles>O=C(CC(=O)c1ccc2ccccc2c1)c1ccccc1</smiles>

50

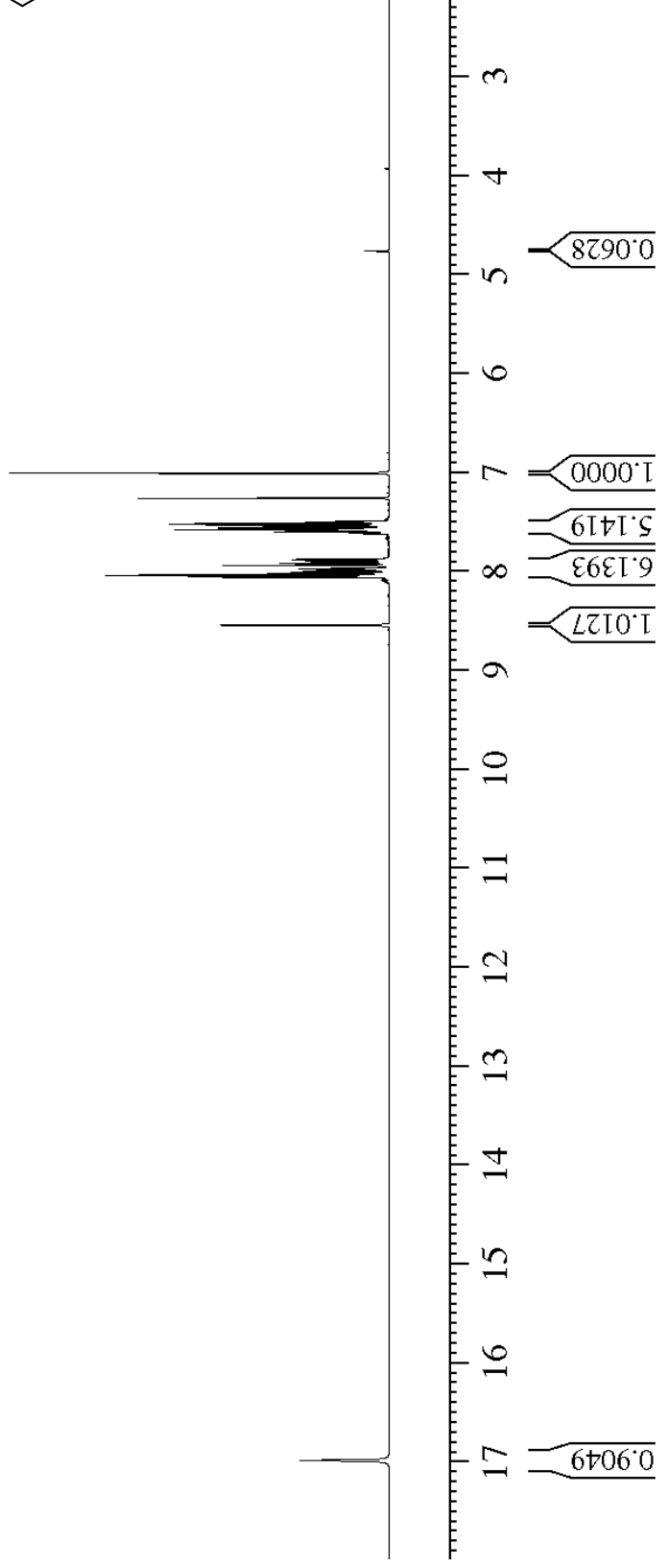




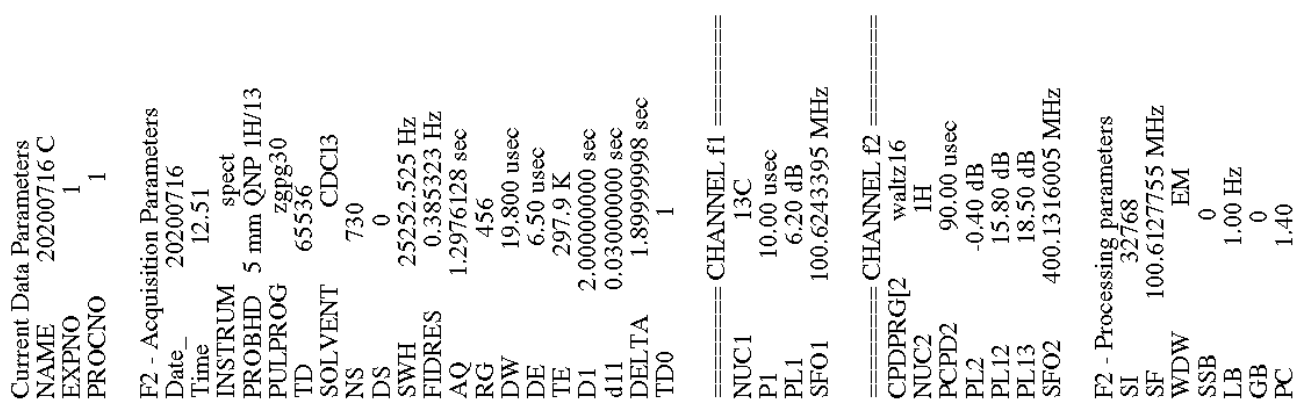

$6289^{\circ} 9 L$

$0000^{\circ} \mathrm{LL}$

$9 \mathcal{C O Z}^{\circ} \mathrm{LL}$

E8I $\mathcal{E}^{\prime} L L$

$28 z t^{\circ} \varepsilon 6$

0 DIZ $\varepsilon Z I$

$8 \$ 9 L^{\circ} 9 Z \mathrm{I}$

6Z8I'LZI

I $8 \nabla L ' L Z I$

99I I' $8 Z \mathrm{I}-$

E00E'8ZI

L9t7 $8 Z \mathrm{ZI}$

SL99.8ZI

$\checkmark \subseteq \mathcal{H}$.6ZI

Et' $Z \mathcal{E I}$

$\angle 8 \mathrm{I} L$ 'ZE I

† $\mathcal{L} L \mathcal{} \cdot Z \mathrm{I}$

$6 \angle 8 Z \subseteq \mathcal{} \subseteq \mathrm{I}$<smiles>O=C(CC(=O)c1ccc2ccccc2c1)c1ccccc1</smiles>

50

LILS'ऽEI

$8 \mathrm{I} 6 \mathrm{t}^{\circ} \mathrm{S} 8 \mathrm{I}-$

$8 \mathrm{TL} \cdot \mathrm{S} 8 \mathrm{I} \longrightarrow$

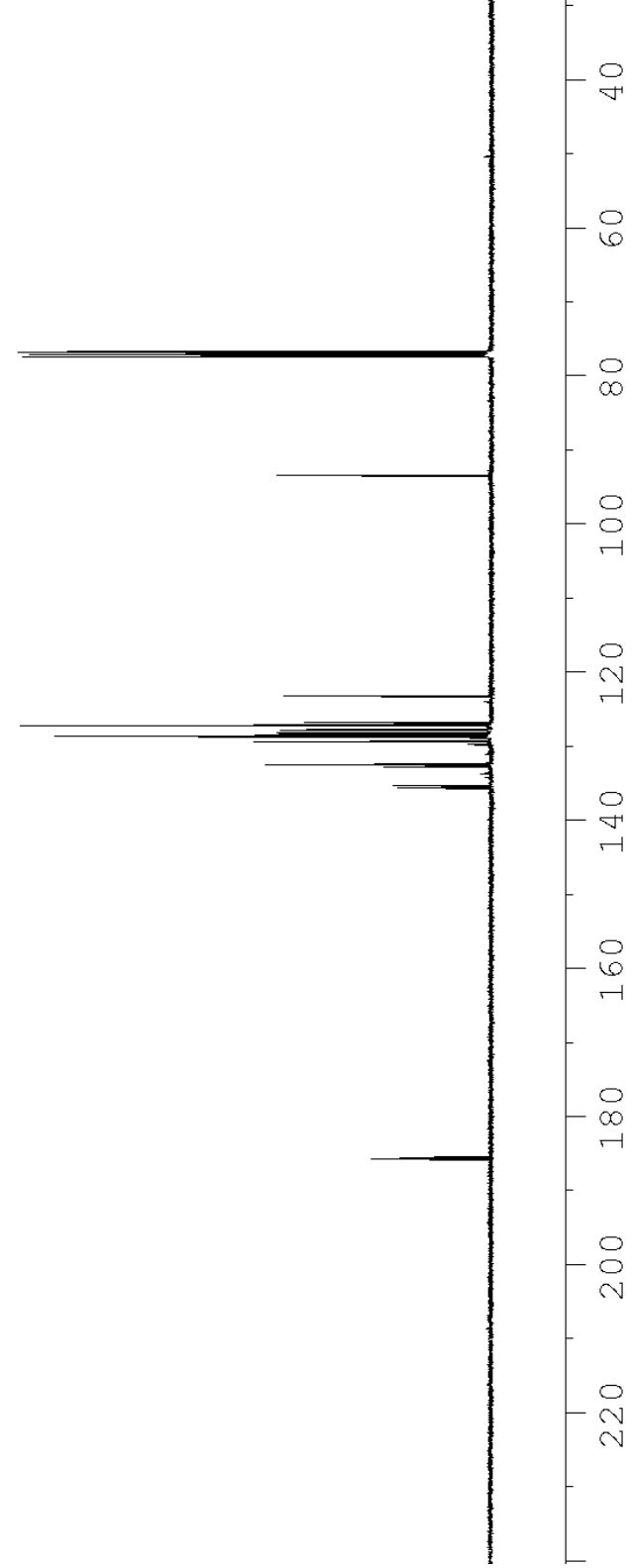




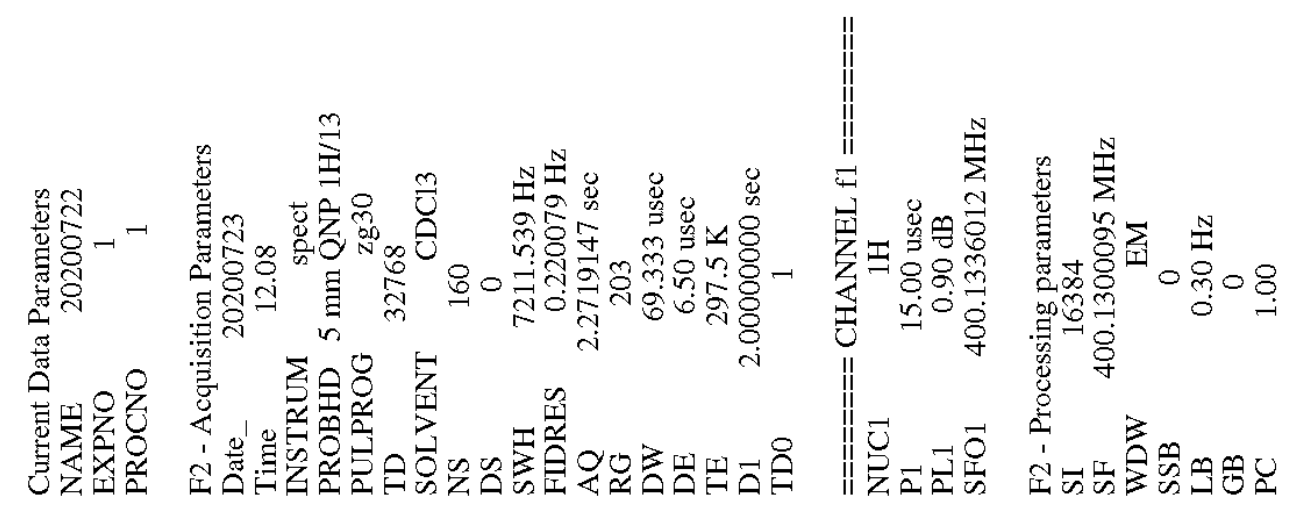<smiles>COc1cccc(C(=O)CC(=O)c2ccc(C)cc2)c1</smiles>

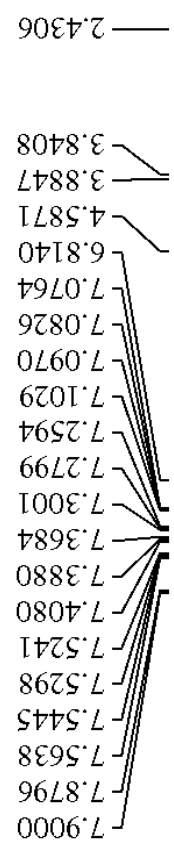

$$
5 p
$$




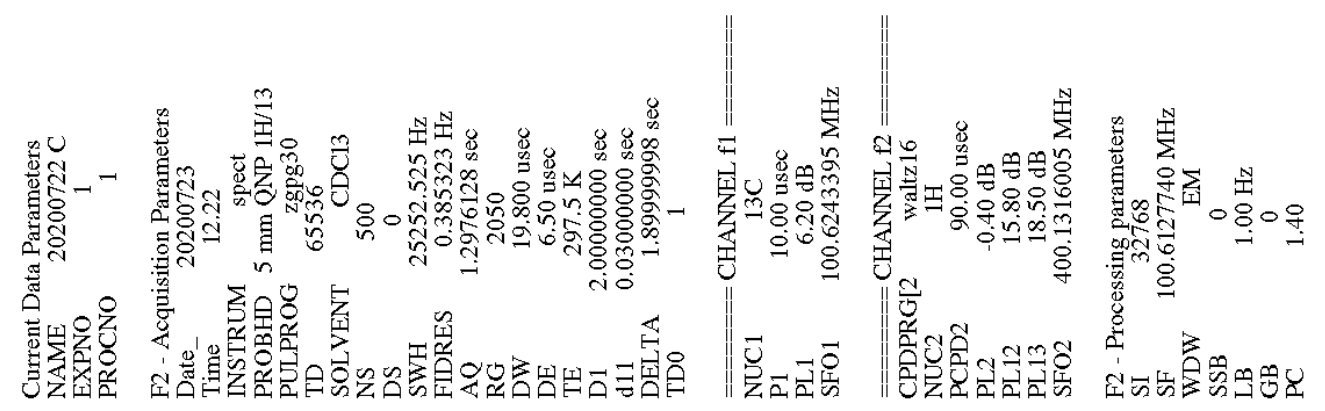

L9I9.IZ

$9 L Z t \cdot S \varsigma$

$\angle 289^{\circ} 9 L$

$0000^{\circ} \mathrm{LL}$

I8IE $L L$

$6+L 6 \cdot 26$

ऽ2Z6. III

$8 \mathrm{IZ} 7^{\circ} 8 \mathrm{II}$

E60S.6I I -

$\$ 96 \mathrm{I}^{\circ} \mathrm{LZI}$

0Е8\& $6 \mathrm{ZI}$ -

$0 \angle 8 S^{\circ} 6 \mathrm{ZI}$

IEI $L$ ZEI

9I0I'LEI -

s89 ' $\mathcal{E} \mathrm{tI}$

$20 \$ 8^{\circ} 6 \mathrm{SI}$

EEEZ $\$ 8 \mathrm{I} \longrightarrow$

$\mathrm{I}$ t0L'S $8 \mathrm{I} \longrightarrow$<smiles>COc1cccc(C(=O)CC(=O)c2ccc(C)cc2)c1</smiles>

$5 p$

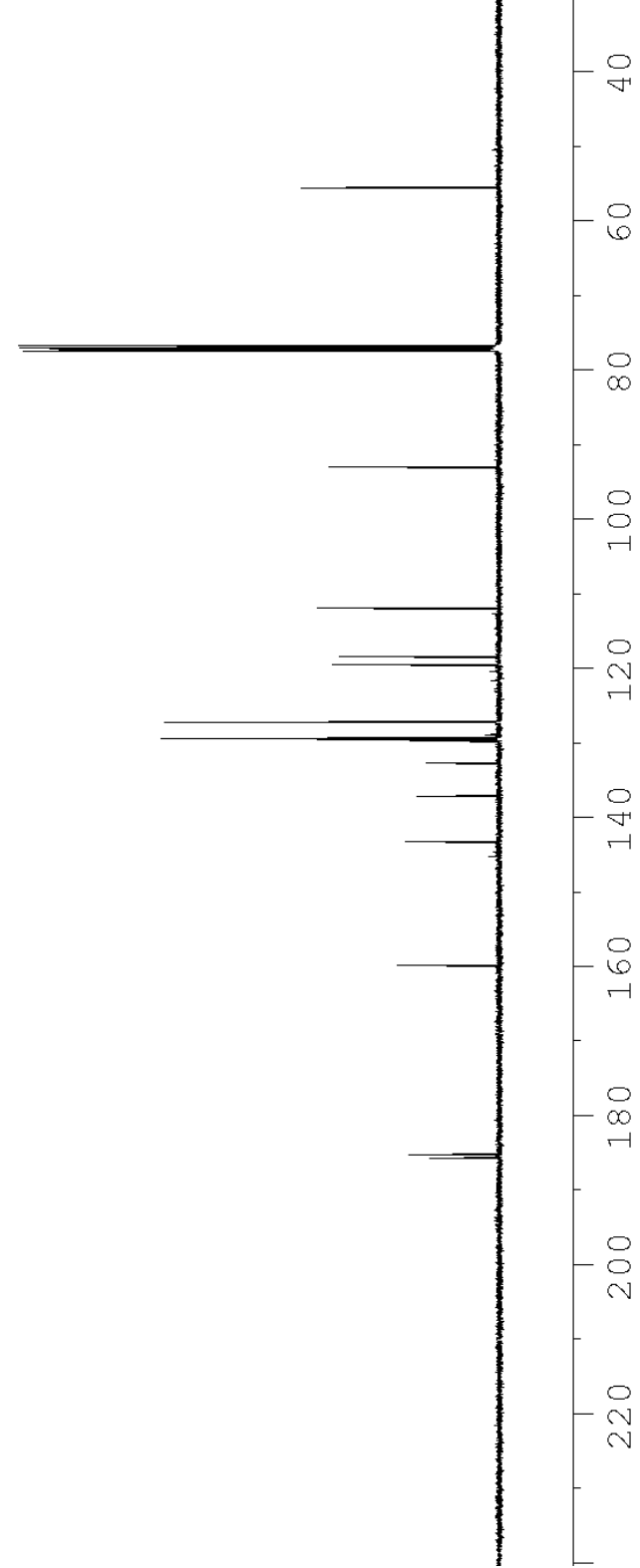




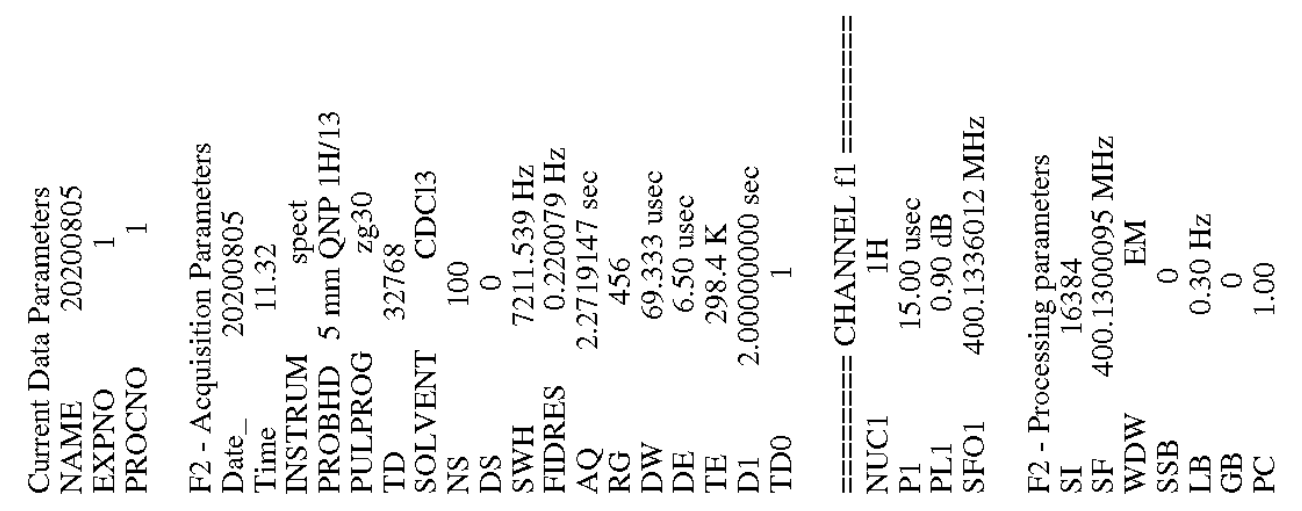

orStr

2S $\angle 9^{\circ} \circ$

0092.

LIIEL

8ZEEL

$8 \varepsilon \angle 9^{\circ} L$

$\angle E 69^{\circ} L$

$\angle E[L \circ]$

$[t[6 . L]$

$\left.9 \nabla \varepsilon 6^{\circ} L\right]$

OLOE 8

IOIE 8

ऽ97ह 8

08LE: 8

$66 L \varepsilon: 8$

IE\&\&: $8-$

[S $8 \varepsilon^{\circ} 8$ -

086E 8

So0t: 8

$\varepsilon \varepsilon 07^{\circ} 8$

$\neg \angle 8 L L^{\circ}$

$026 L^{\circ} 8$

L96L:8<smiles>Cc1ccc(C(=O)CC(=O)c2cccc([N+](=O)[O-])c2)cc1</smiles>

$5 q$

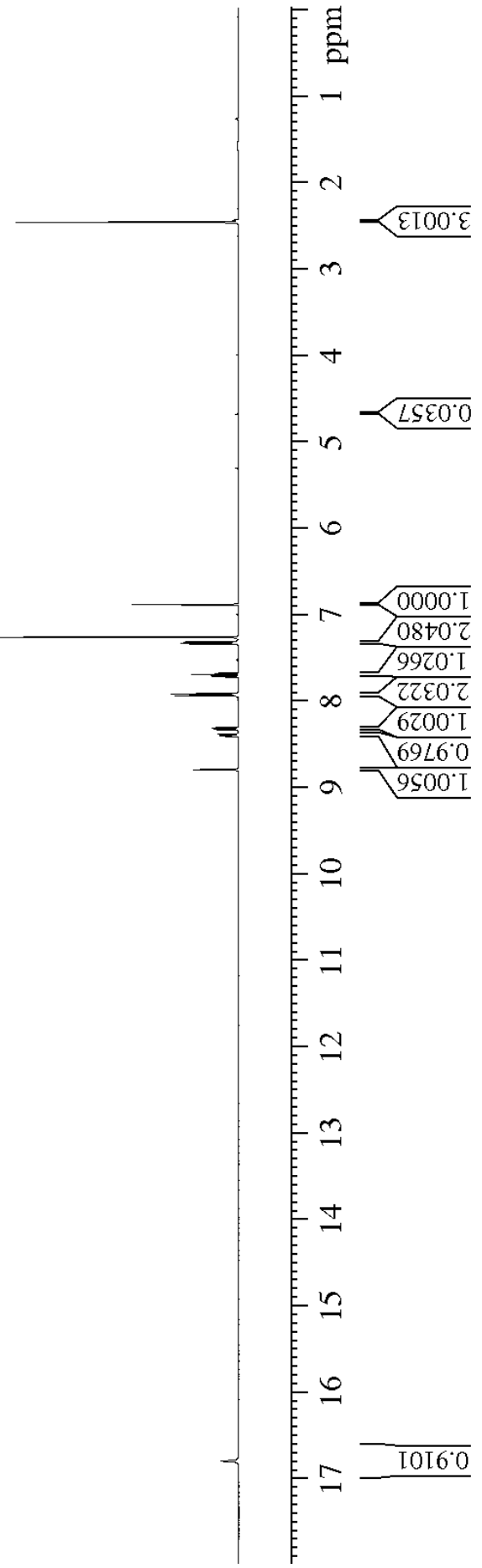




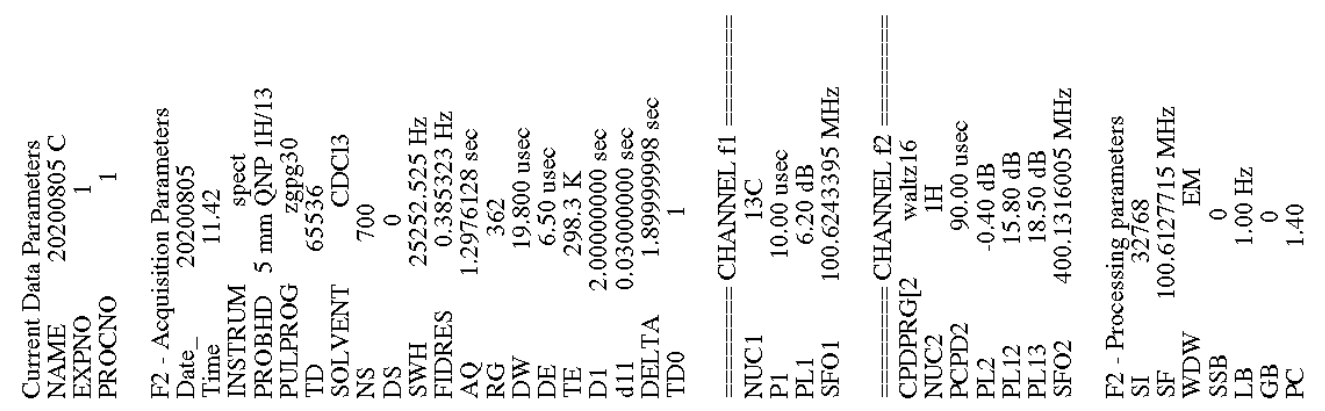

$860 L^{\prime} \mathrm{IZ}$<smiles>Cc1ccc(C(=O)CC(=O)c2cccc([N+](=O)[O-])c2)cc1</smiles>

$5 q$

$0 \varepsilon 89^{\circ} 9 L$

$0000^{\circ} \mathrm{LL}$

$890 I^{\circ} \mathcal{E} 6$

SESt.9ZI

09St LZI

IE9S"6ZI

tIS8.6ZI

690Z ZEI

$90 \mathcal{L} L Z \mathcal{} \cdot \mathcal{I}$

$3 \varepsilon 9 \mathcal{E}^{\prime} L \mathcal{} L \mathrm{I}$

$6 L 90^{\circ} \mathrm{t} \mathrm{I}$

$0 L L D^{\circ} 8 \mathrm{I}-$

L686 I8I

$6+\angle 6^{\circ} 98 \mathrm{I}$

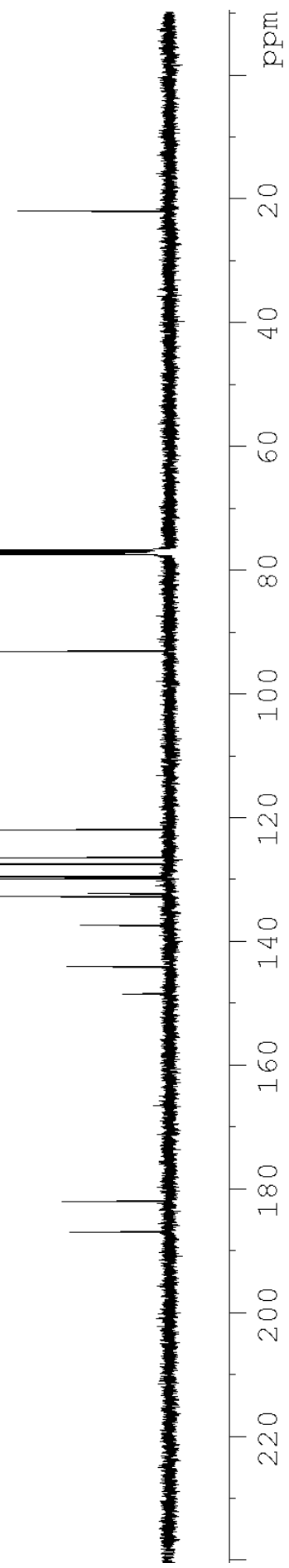




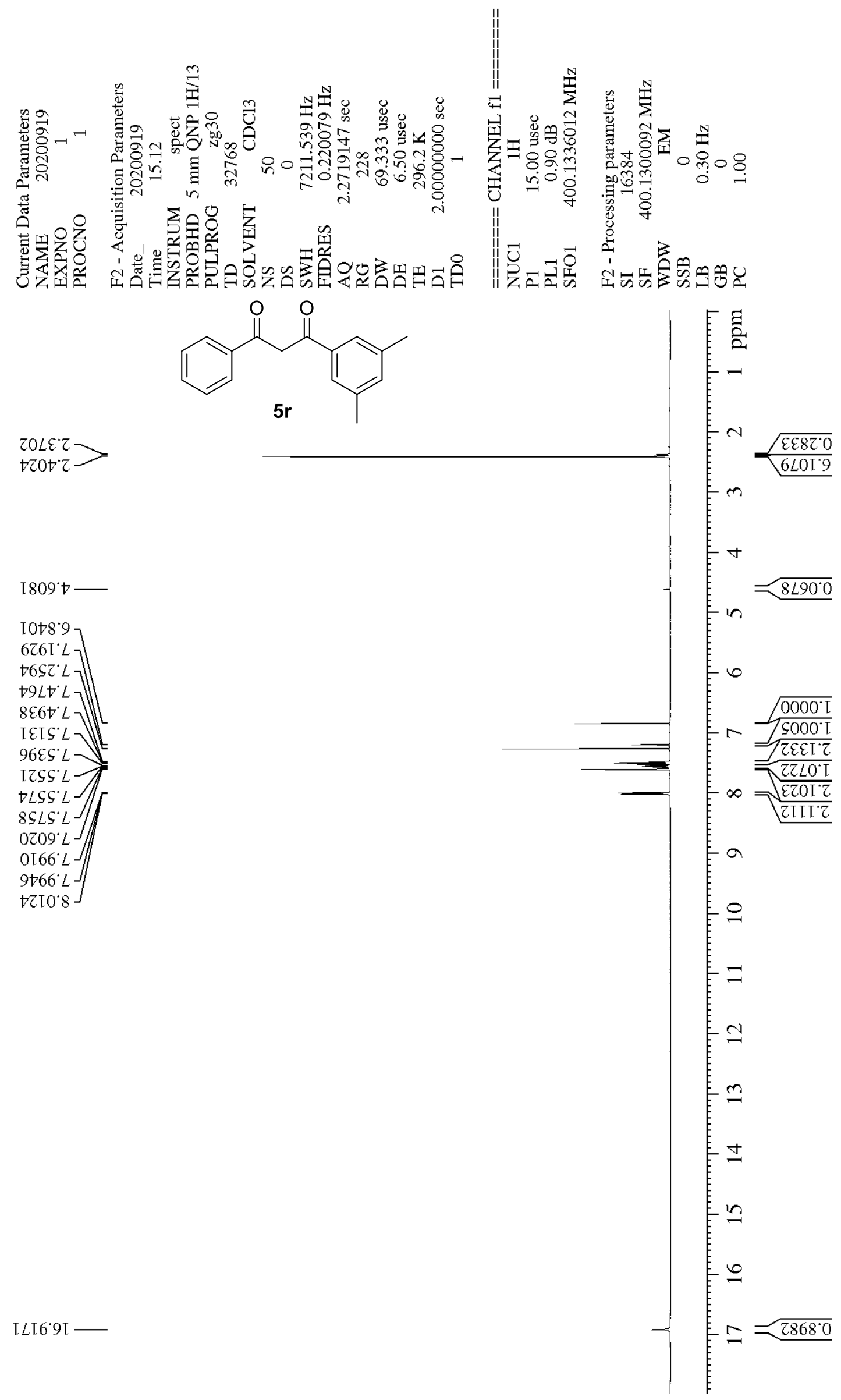




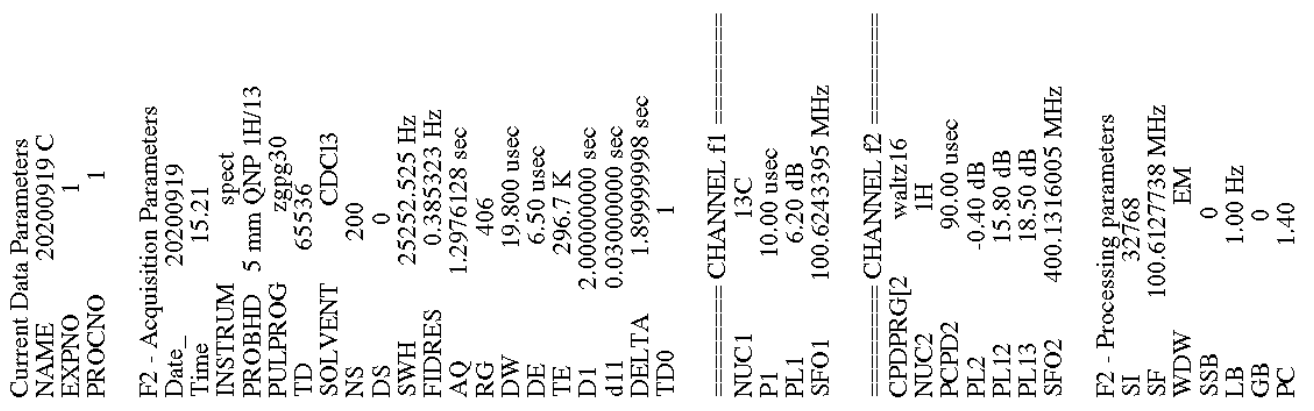

เ8LZ:IZ<smiles>Cc1cc(C)cc(C(=O)CC(=O)c2ccccc2)c1</smiles>

IE89.9L

$0000^{\circ} \mathrm{LL}$

I8IE $L L$

$\angle S_{0 Z} \& 6$

¿E6 ${ }^{\circ} \mathrm{TI}$

$8 S Z I^{\prime} L Z I$

8SZ9.8ZI

JIEE'ZEI

† $6 \mathrm{I} \cdot \mathrm{t} \varepsilon \mathrm{I}$

DOOS'SEI T

$6209^{\circ} \mathrm{SEI}$

$686 Z 8 \& \mathrm{I}$

$\$ 887^{\circ} \mathrm{S} 8 \mathrm{I}-$

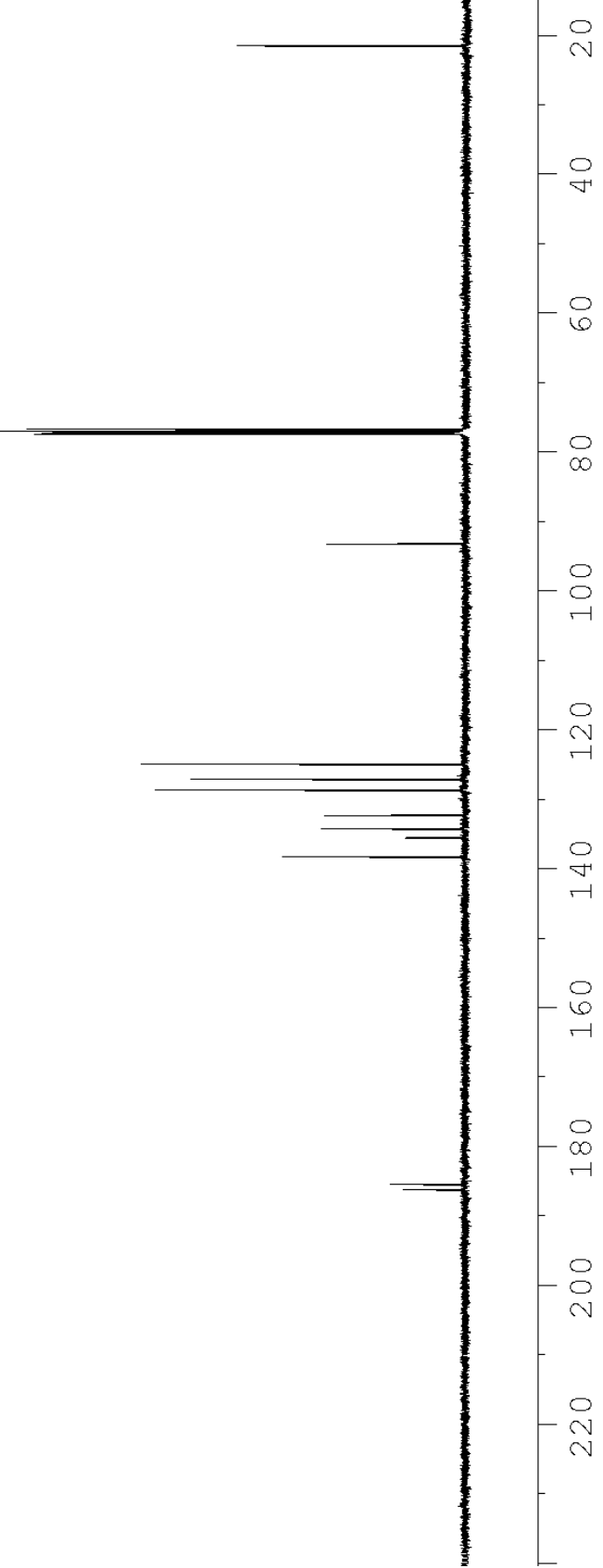



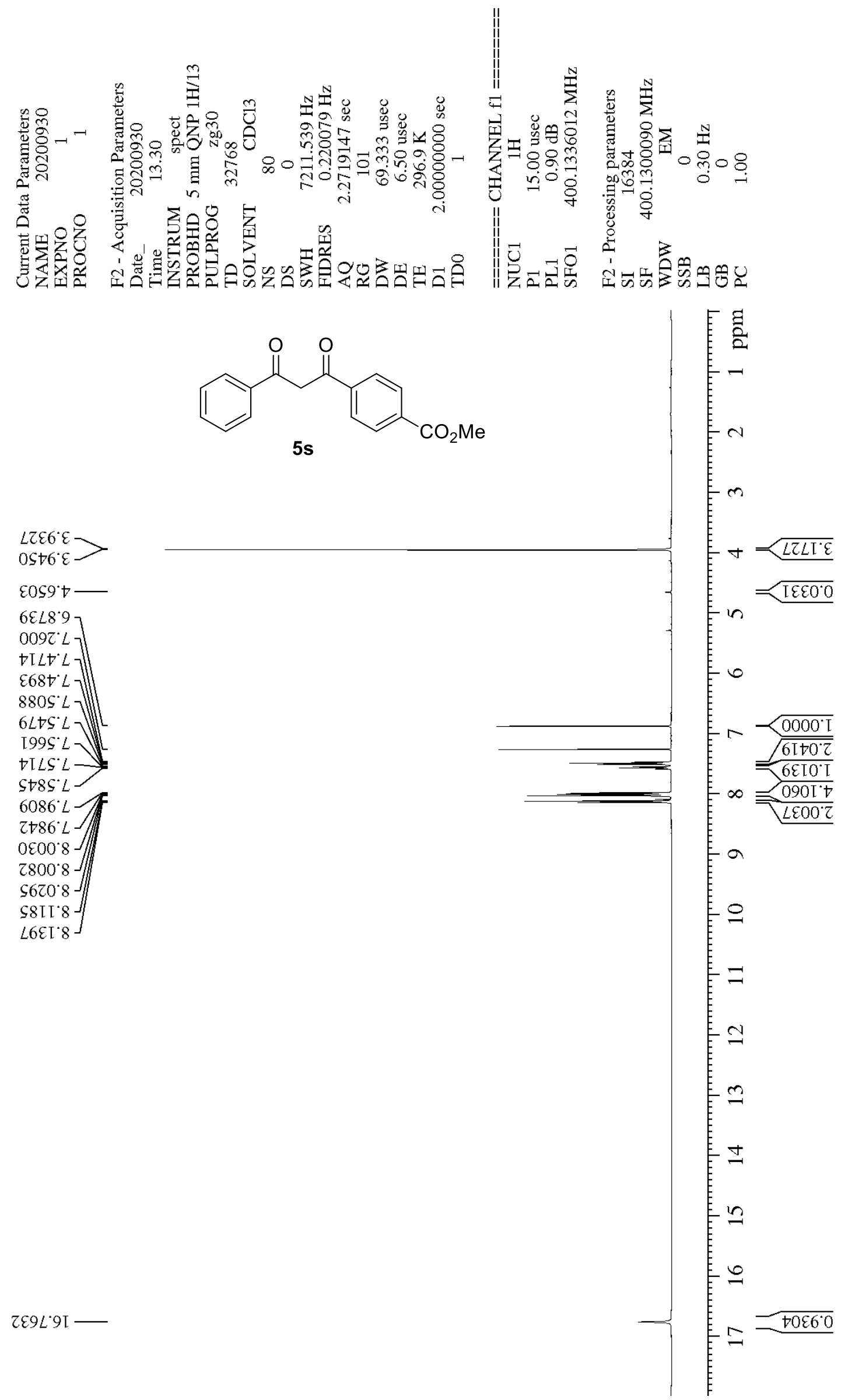


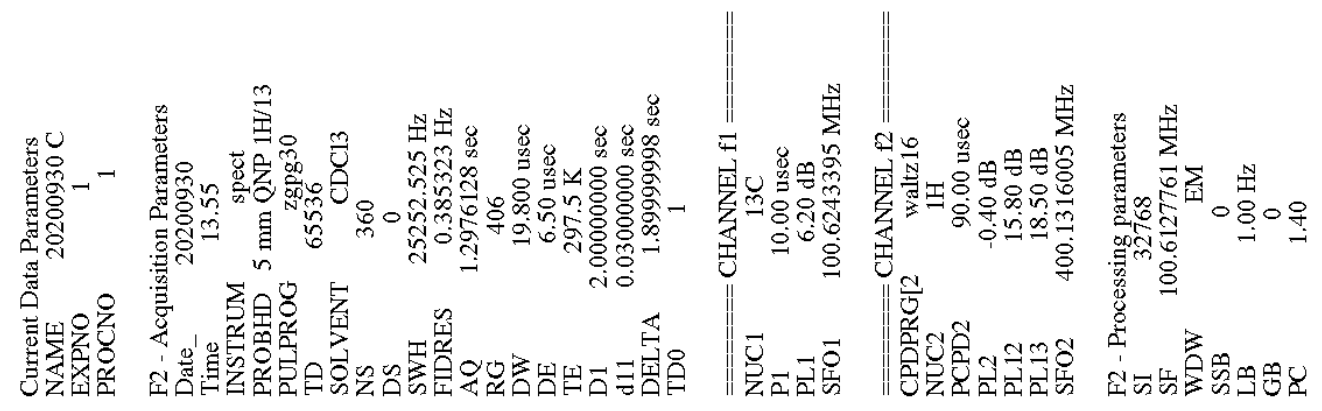<smiles>COC(=O)c1ccc(C(=O)CC(=O)c2ccccc2)cc1</smiles>

$\exists L S \mathcal{Z} Z S$

$9789^{\circ} 9 L$

$0000^{\circ} L L$

$08 I E \cdot L L$

5s

ItSLL' $\mathcal{E}_{6}$

$\varepsilon 0 L 6^{\circ} 9 \mathrm{ZI}$

I892 LZI

†ย69.8ZI -

$\varepsilon \varepsilon 8 L^{\circ} 6 \mathrm{ZI} \longrightarrow$

IS $D L ' Z E I$

$\checkmark S L I ' \mathcal{E}$ I -

¡EEE' $\subseteq E I$

II $\angle I$ ' $6 \varepsilon$ I

stzZ:99I

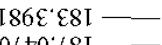

$0 L t 0 . \angle 8 \mathrm{I}$

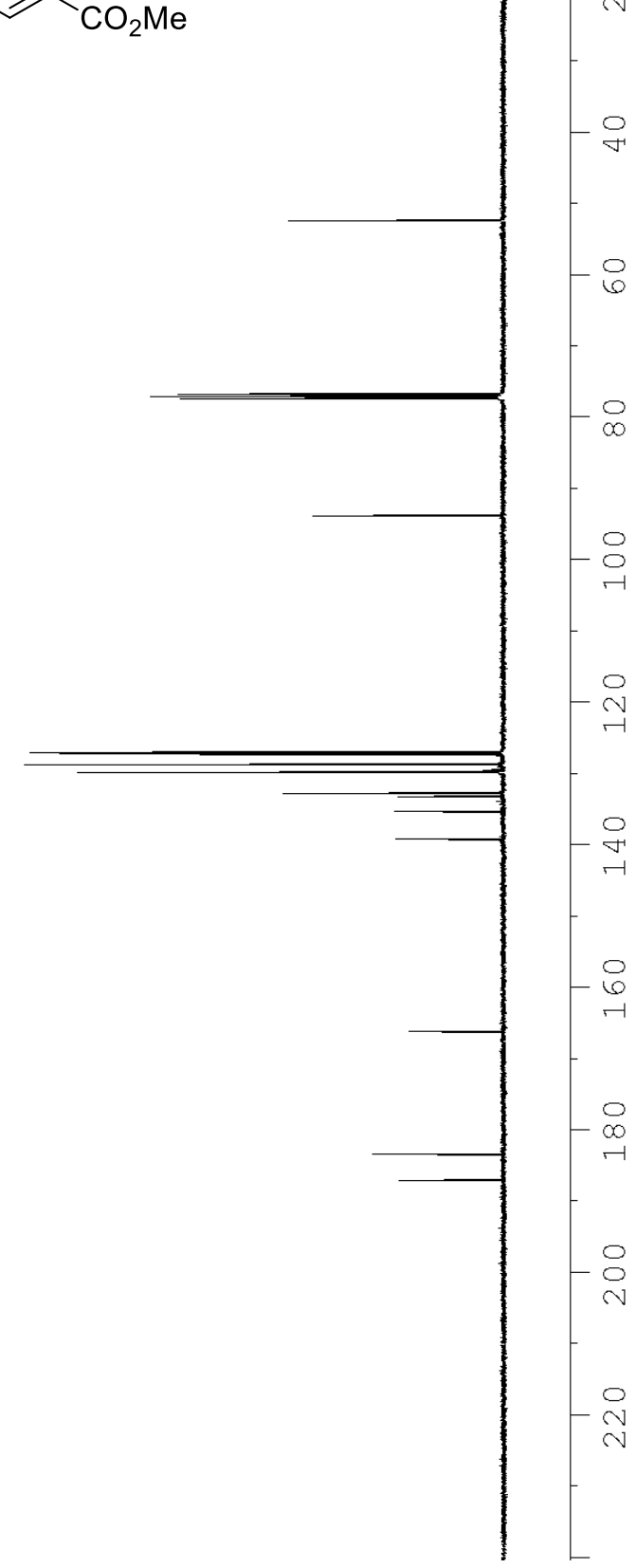


2) ${ }^{1} \mathrm{H}$ and ${ }^{13} \mathrm{C}$-NMR Spectra of $7 / 8$

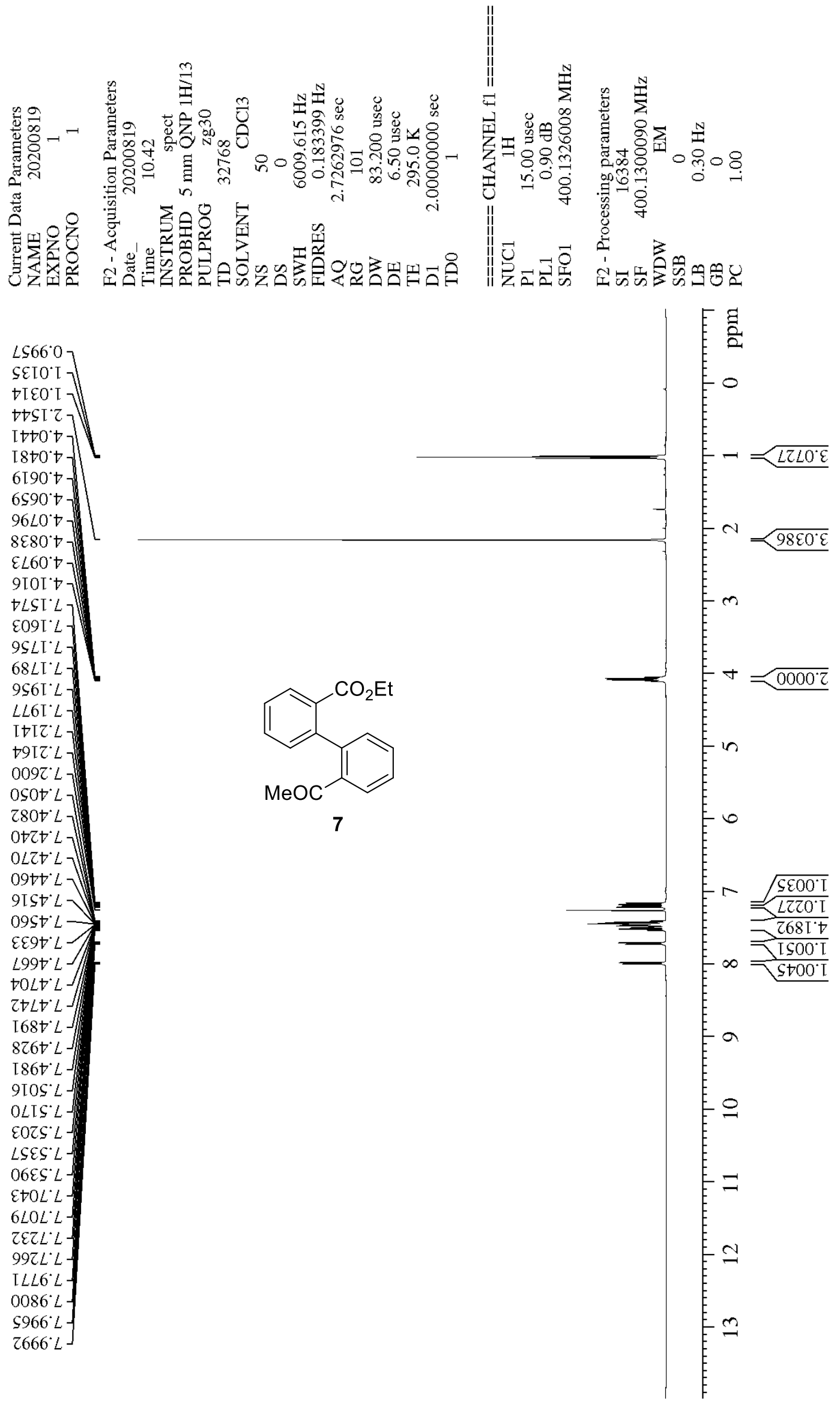



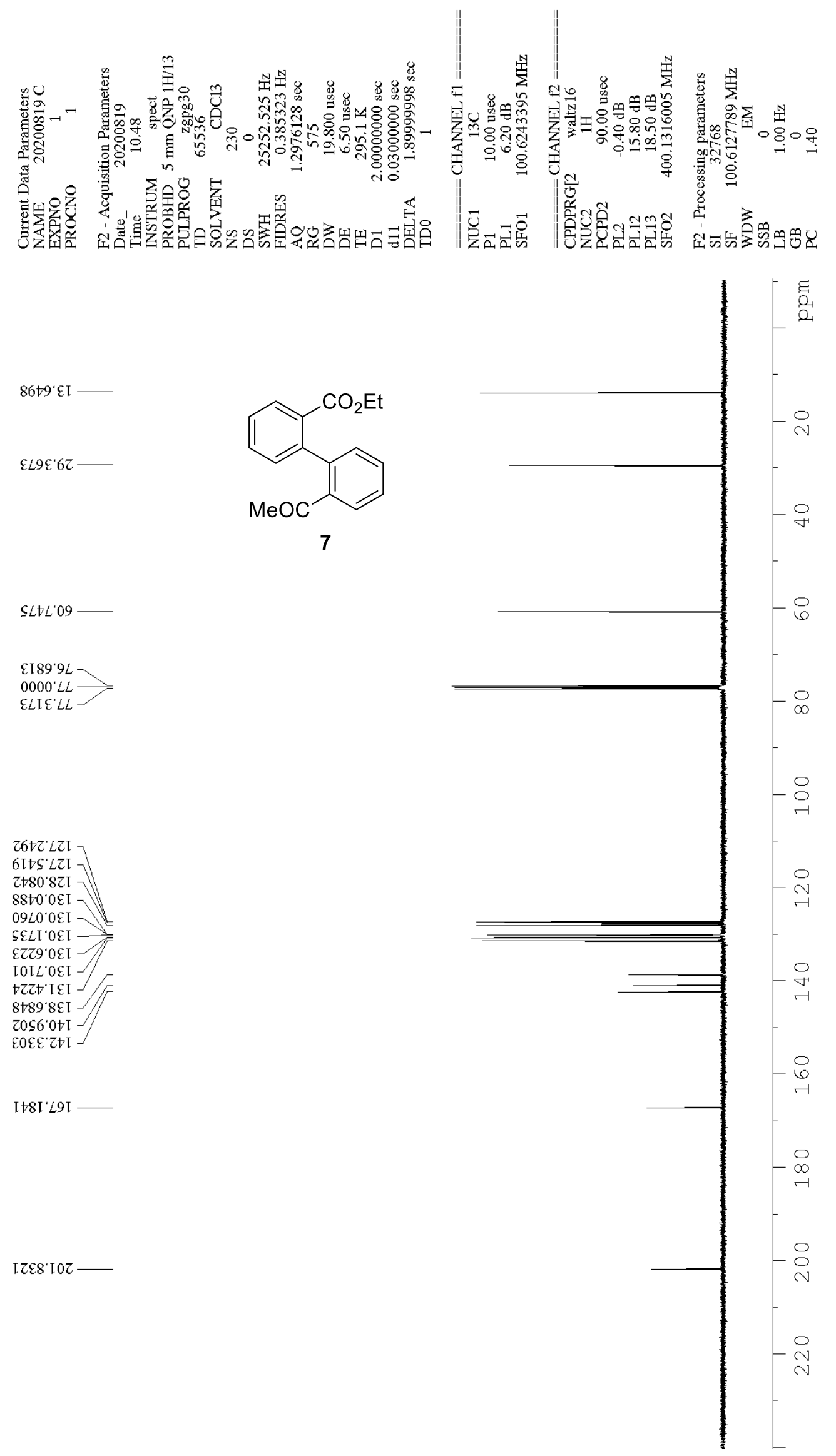


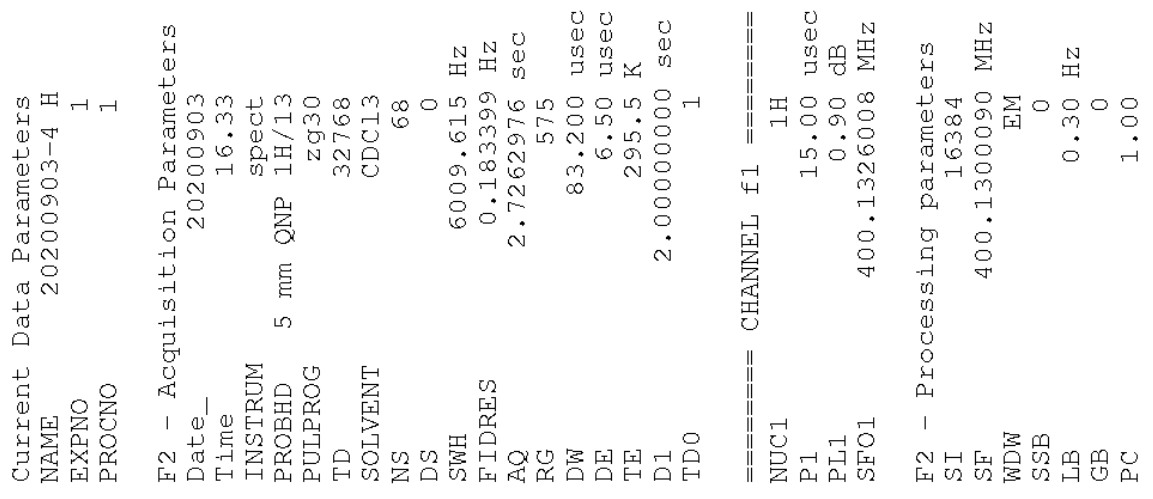

$007 \tau \cdot 7$

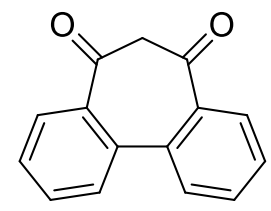

8
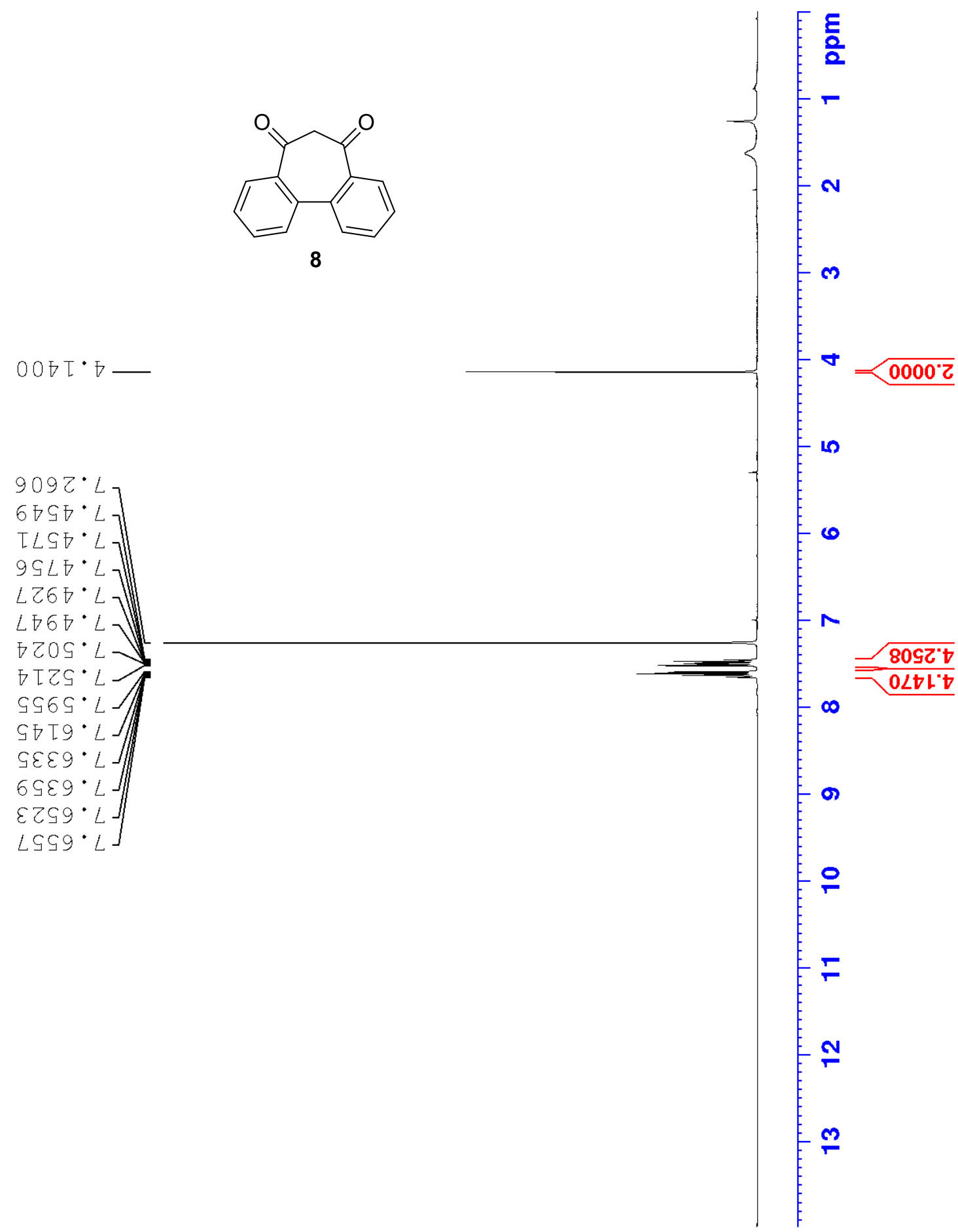

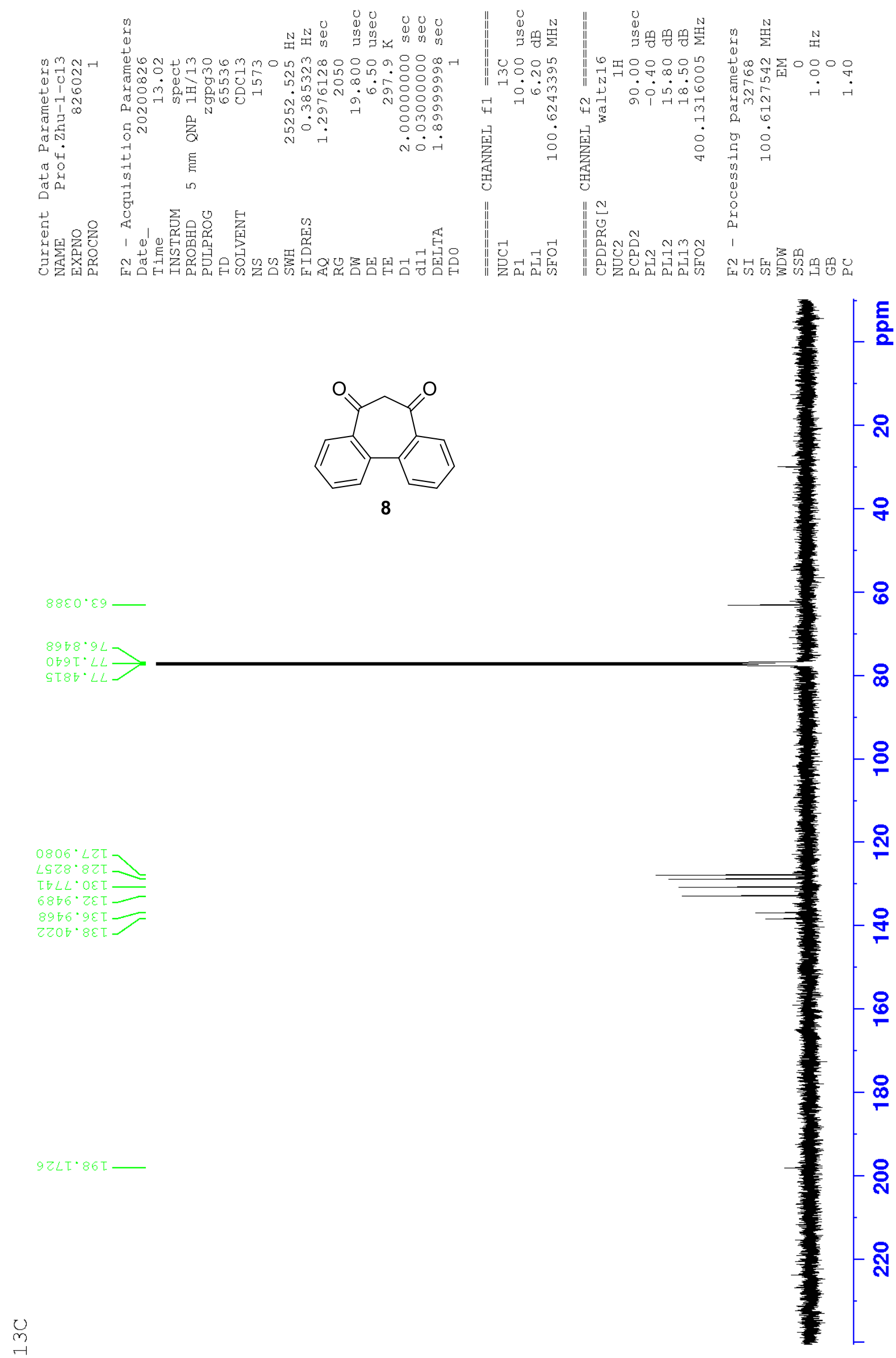


\section{3) $1 \mathrm{H}$ and $13 \mathrm{C}-\mathrm{NMR}$ spectra of 1a-u}

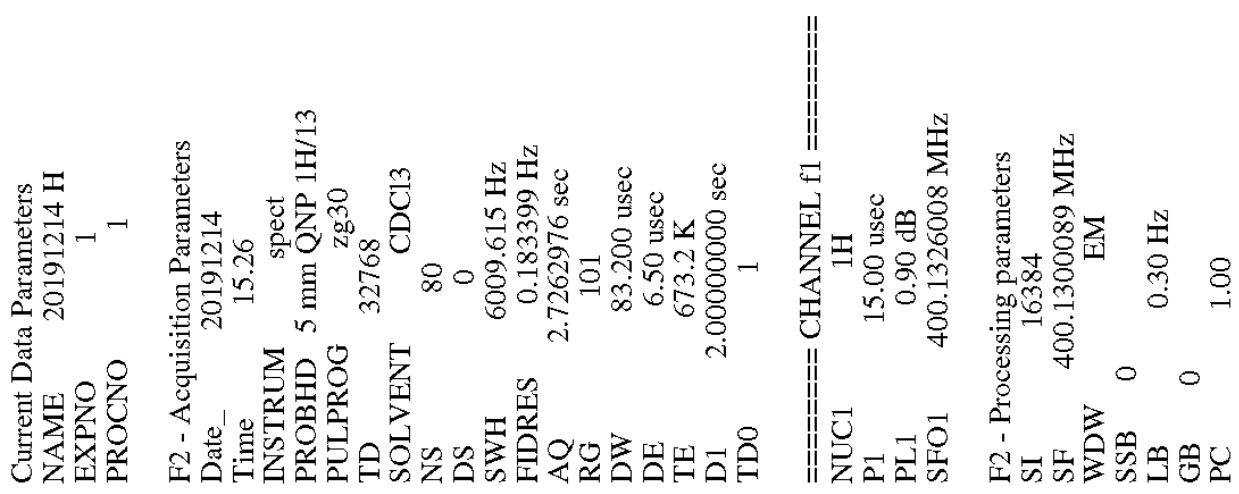

9E9 : $L$

$0 z 8 \mathrm{I}^{\circ} \mathrm{L}-$

t09 $: L$

$8 \mathrm{~L} L \tau^{\circ} L$

$806 \tau^{\circ} L$

$060 \varepsilon L$

$0+\tau \varepsilon L$

$+97 \varepsilon L$

$\downarrow \varepsilon \varepsilon \varepsilon L$

$\dashv 6 \varepsilon \varepsilon L-$

$\angle 8 t \varepsilon: L$

$\angle L S E L$

$\varepsilon 89 \varepsilon \varepsilon_{7}$

¿9LE $L$

$S t 8 \varepsilon: L$

I 6 6E $L-$

†96E $L$

OLOt $L$

$L Z Z \nabla^{\circ} L-$

ISZT: $L$

6IEt $L$

IItt $L$

$\varepsilon 0 S T^{\circ} L$

$S E S T L$

L6St $L$

$\angle 89 t^{\circ} L$

IZLT $L-$

$\angle 687^{\circ} L-$

$8 \varepsilon 6 t^{\circ} \mathrm{L}$

$\checkmark 80 S^{\circ} \mathrm{L}-$

$\rightarrow$ IIS $L-$

EOES $L_{-}$

$\nabla \varepsilon \varepsilon S^{\circ} L-$

$98+S^{\circ} L-$

IZSS $L$<smiles>N=C(C(=O)c1ccccc1)C(=O)c1ccccc1-c1ccccc1</smiles>

1a

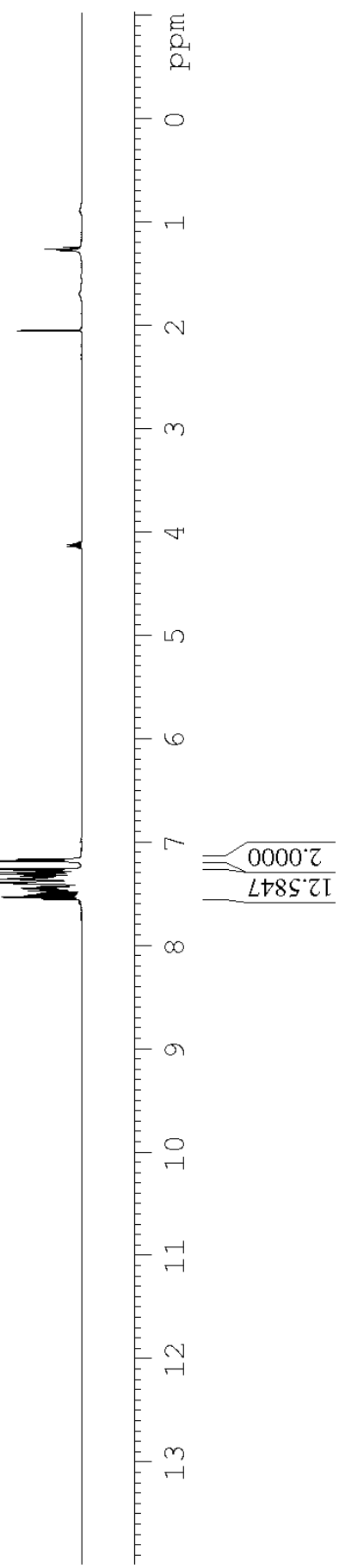




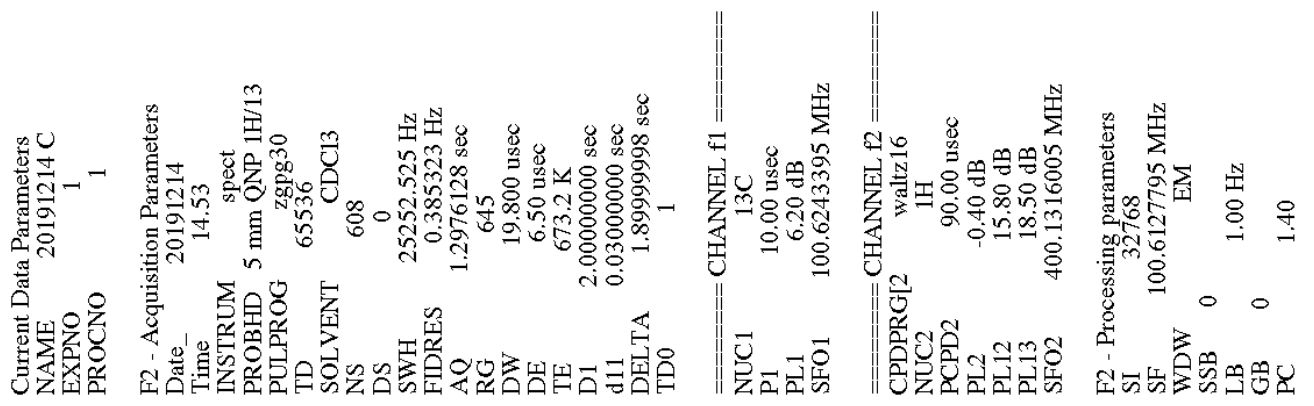

$7 t 89^{\circ} 9 L$

9I00 LL

Z0ZE' $L L$

$\angle S 68^{\circ}+8$<smiles>N=C(C(=O)c1ccccc1)C(=O)c1ccccc1-c1ccccc1</smiles>

$1 a$

$6669^{\circ} \angle Z \mathrm{I}$

$\mathcal{E}$ I $S 8^{\circ} \angle Z \mathrm{I}$

I $8 S^{\circ} \mathrm{LZI}$

0 IS0 8 ZI

$00 / t^{\circ} 8 \mathrm{I}$

I I6I'6ZI

$980 L^{\circ} 6 Z \mathrm{ZI}$

8I90'IEI

6†9t ZEI

6LOE'9EI

6LZ๐ $L E I$

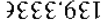

$0 Z 98^{\circ} 6 \varepsilon \mathrm{I}$

I I9L'十8I -

Z† $\angle 8^{\circ} 88 \mathrm{I}$ 


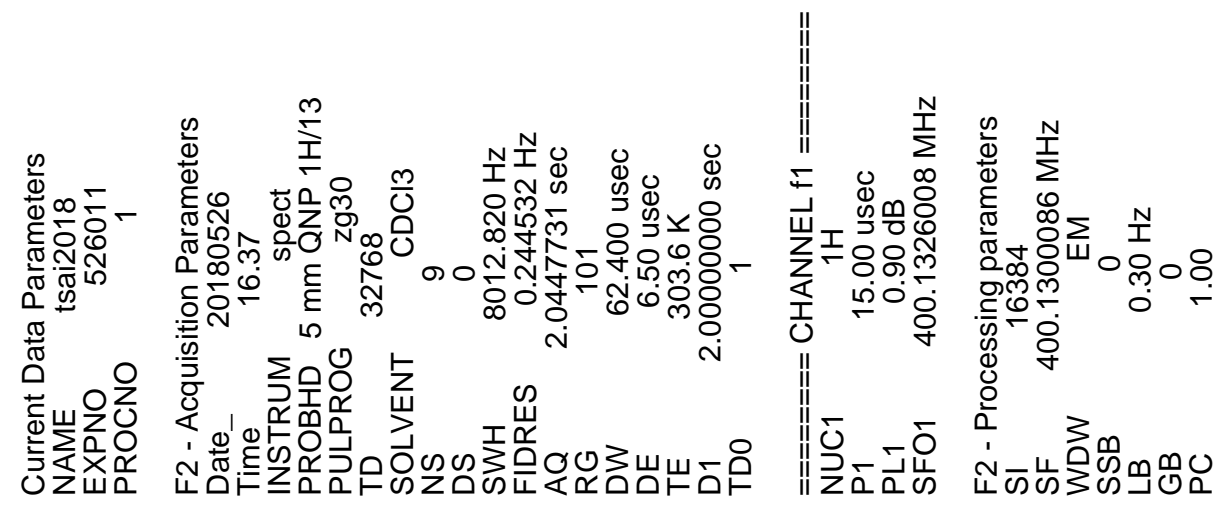<smiles>N=C(C(=O)c1ccccc1)C(=O)c1ccccc1</smiles>

1b

IC8Z' $\angle$

$600 \varepsilon^{\circ} L$

टOटह

8E0t

ยटZヤ L

こLt大 $L$

09SG $L$

$O \forall \angle G^{\circ} \angle$

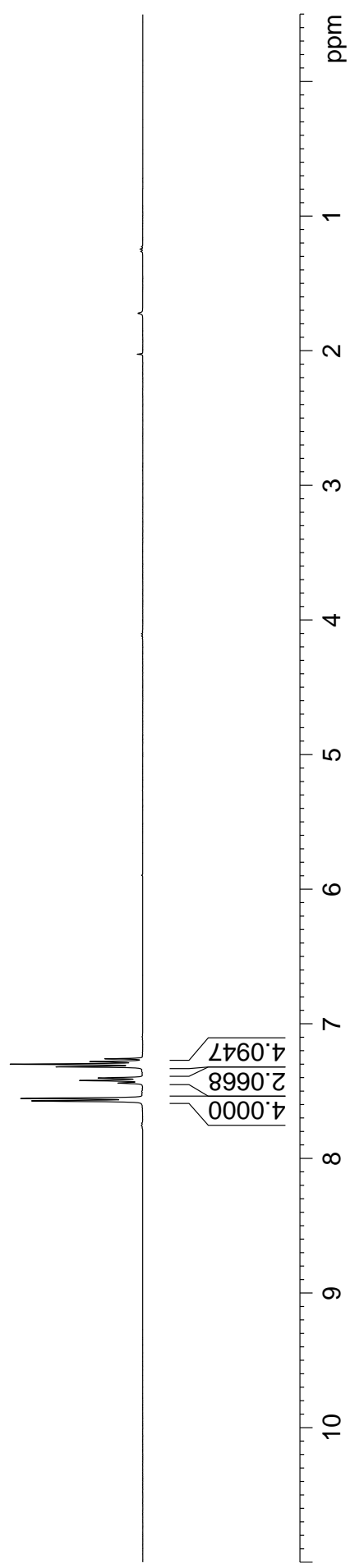




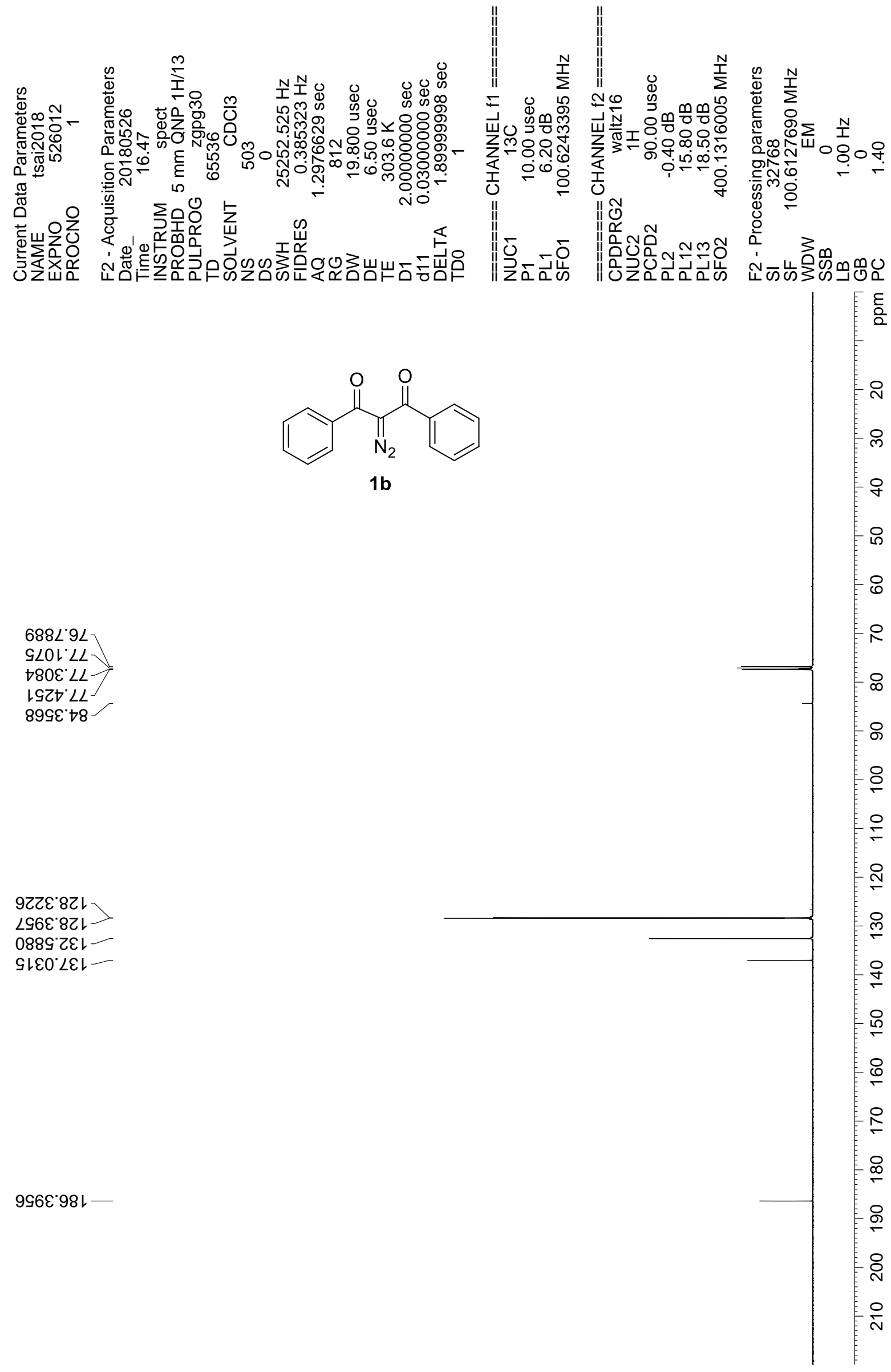




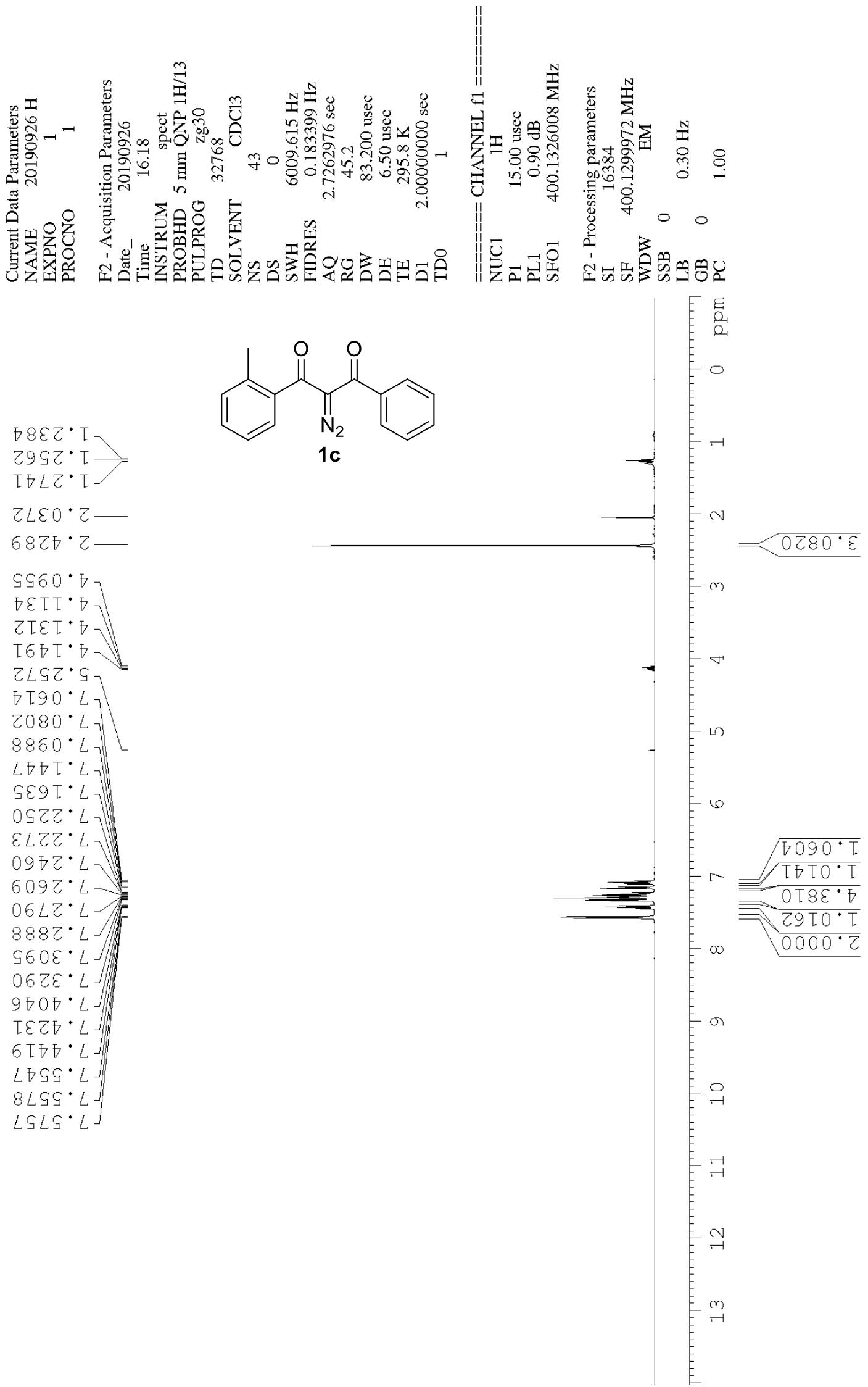




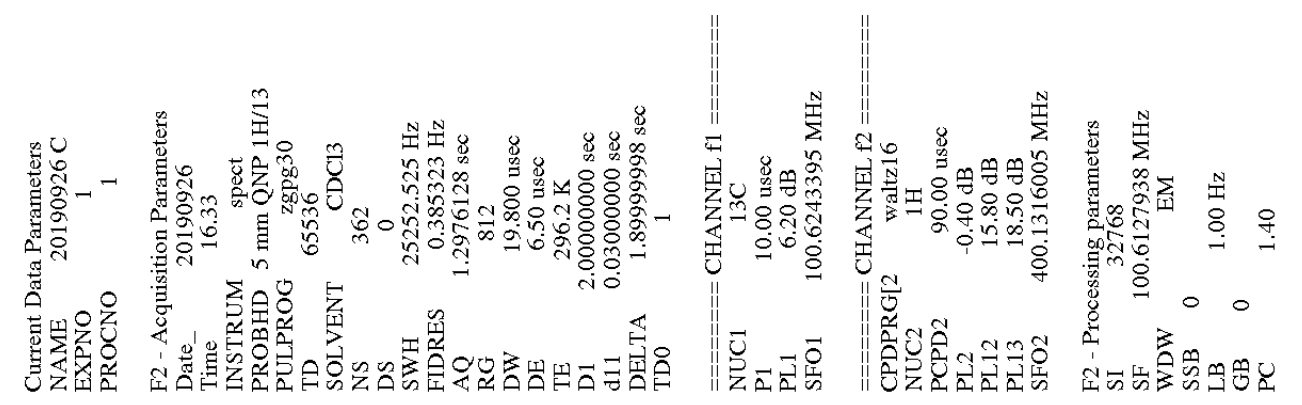

$9 L \nabla Z \cdot 6 I$<smiles>Cc1ccccc1C(=O)C(=N)C(=O)c1ccccc1</smiles>

TS89.9L

$0700^{\circ} L$

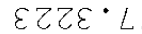

टधৎ8.98

Iร6乙 $\cdot \mathrm{\tau T}$

I692 $\angle 2 T$

8 IS6 $\cdot$ LZT

$\angle 796^{\circ} \angle Z I$

$\angle 80 L^{\circ} 0 \varepsilon T$

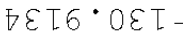

$09 L z \cdot \tau \varepsilon I$

Ђ $8 \varepsilon \sigma^{\circ} \mathrm{G \varepsilon}[-$

$8[\varepsilon L \cdot 9 \varepsilon \tau]$

$0890 \cdot$ LET

$\angle 6 \varepsilon 6 \cdot 98 I_{-}$

$\angle L 89^{\circ} \angle 8 I=$

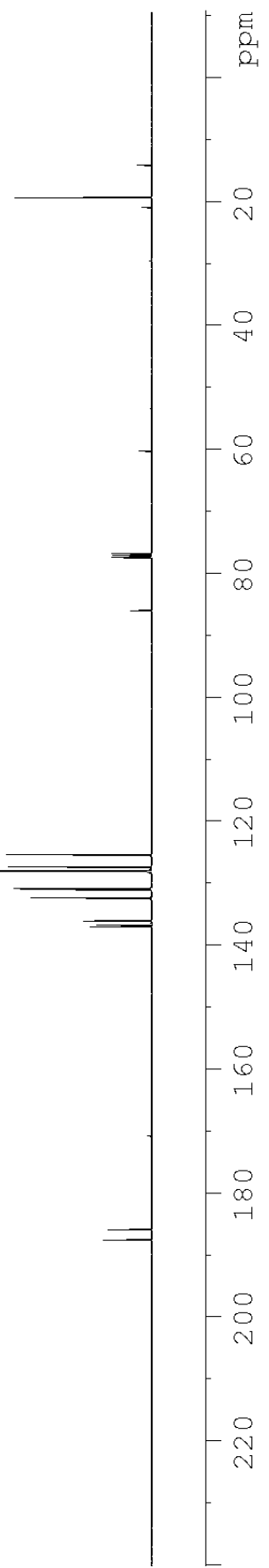




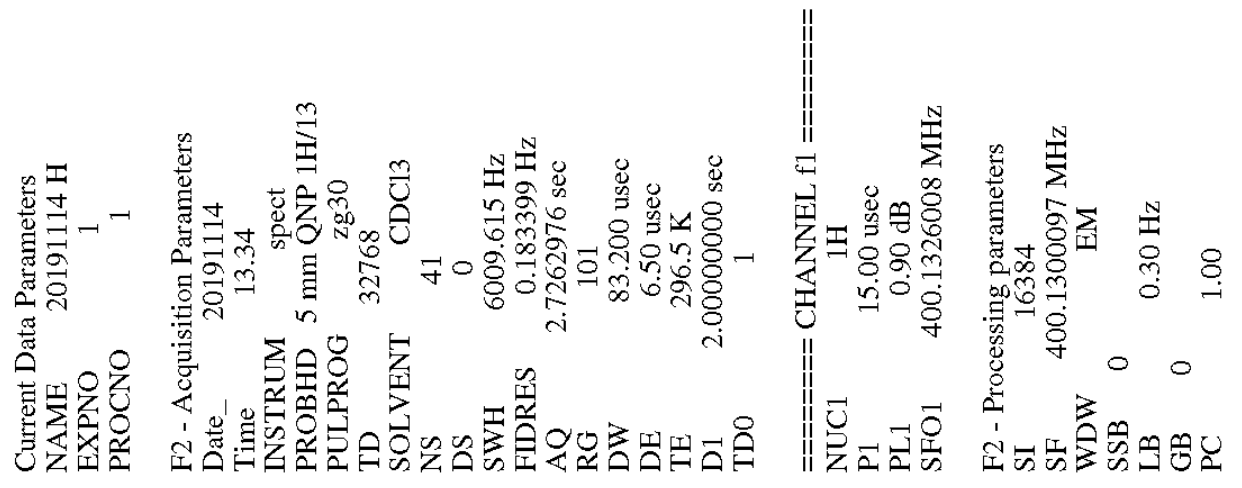

†IT' $L$

66I I $L$

EIEZ $L$

89E2 $L$

I6EZ $L$ -

$\angle E S{ }^{\circ} L-$

E097 $L$

$96 \angle Z^{\circ} L$

$606 \mathrm{C}^{\circ} \mathrm{L}$

†IOE $L$

IOZE'L

$99 \mathcal{E} \mathcal{E}^{\circ}$

S6EE $L$

tDIt $L$

OEEt $L$

LISt' $L$

IS9S $L$

989 ' $L$

2985 $L$<smiles>N=C(C(=O)c1ccccc1)C(=O)c1ccccc1Cl</smiles>

1d

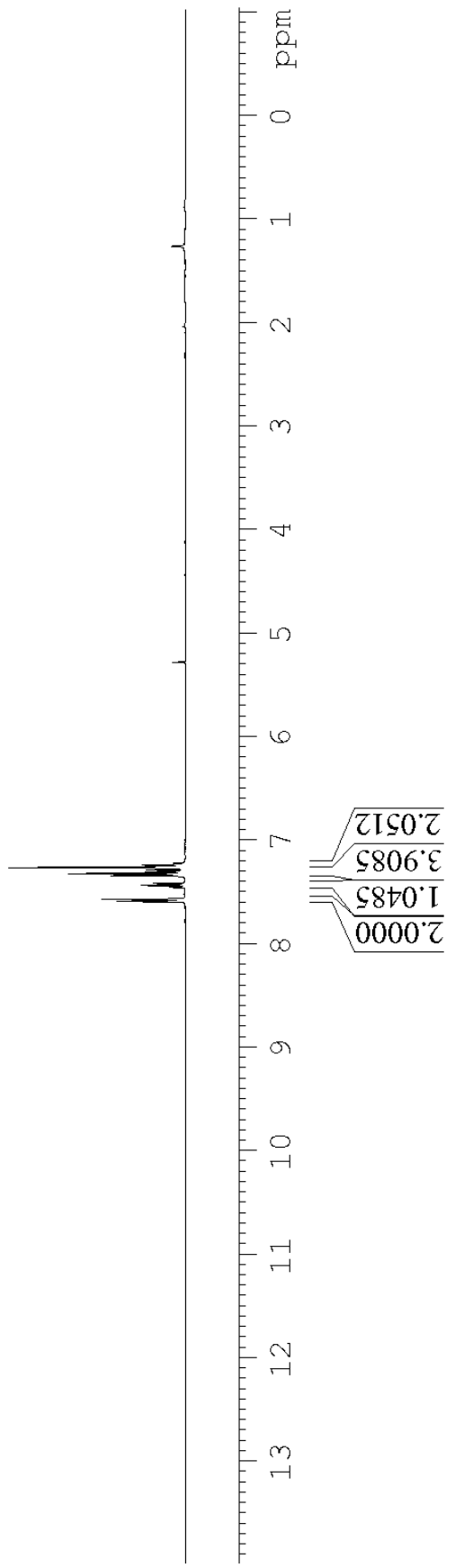




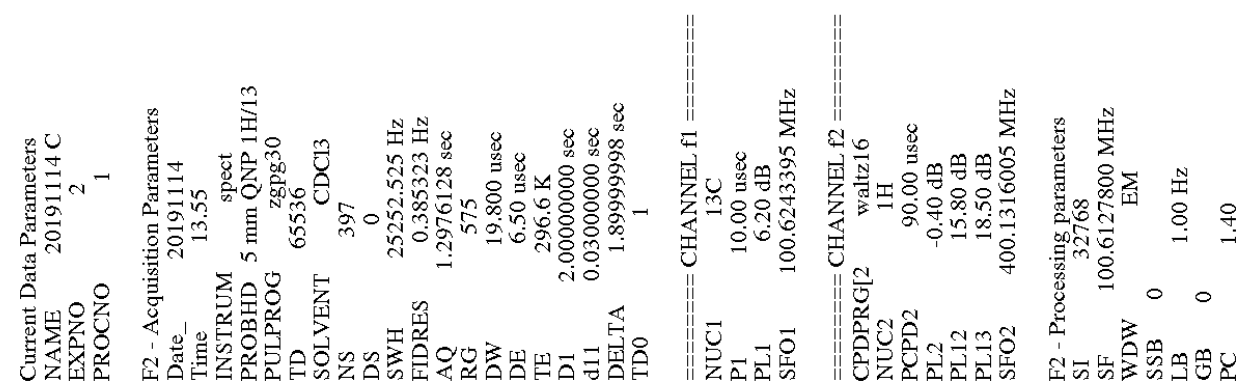

$8 \$ 89^{\circ} 9 L$

$\varepsilon+00^{\circ} \mathrm{LL}$

$8 \mathrm{SOZ}^{\circ} L L$

8IZELL

6IZS 98

$96066^{\circ} 9 \mathrm{I}$

090I'8ZI

$986 \mathrm{I}^{\circ} 8 \mathrm{ZI}$

$\angle t \$ 8^{\circ} 8 Z \mathrm{I}$

6Z9L6ZI

SI69. OEI

L Z L IEI

689ऽ ZEI

OLL $9 \varepsilon \mathrm{I}$

ZS8Т $L E \mathrm{I}$

$\varepsilon 6 \mathrm{ZZ} \varsigma 8 \mathrm{I}$

$\varepsilon \mathrm{I} 8 \varepsilon \subseteq 8 \mathrm{I} \longrightarrow$<smiles>N=C(C(=O)c1ccccc1)C(=O)c1ccccc1Cl</smiles>

1d

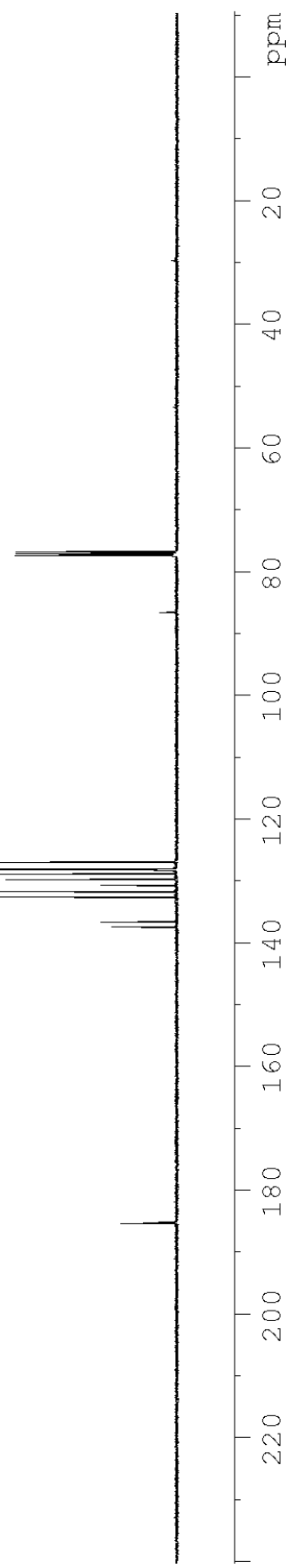




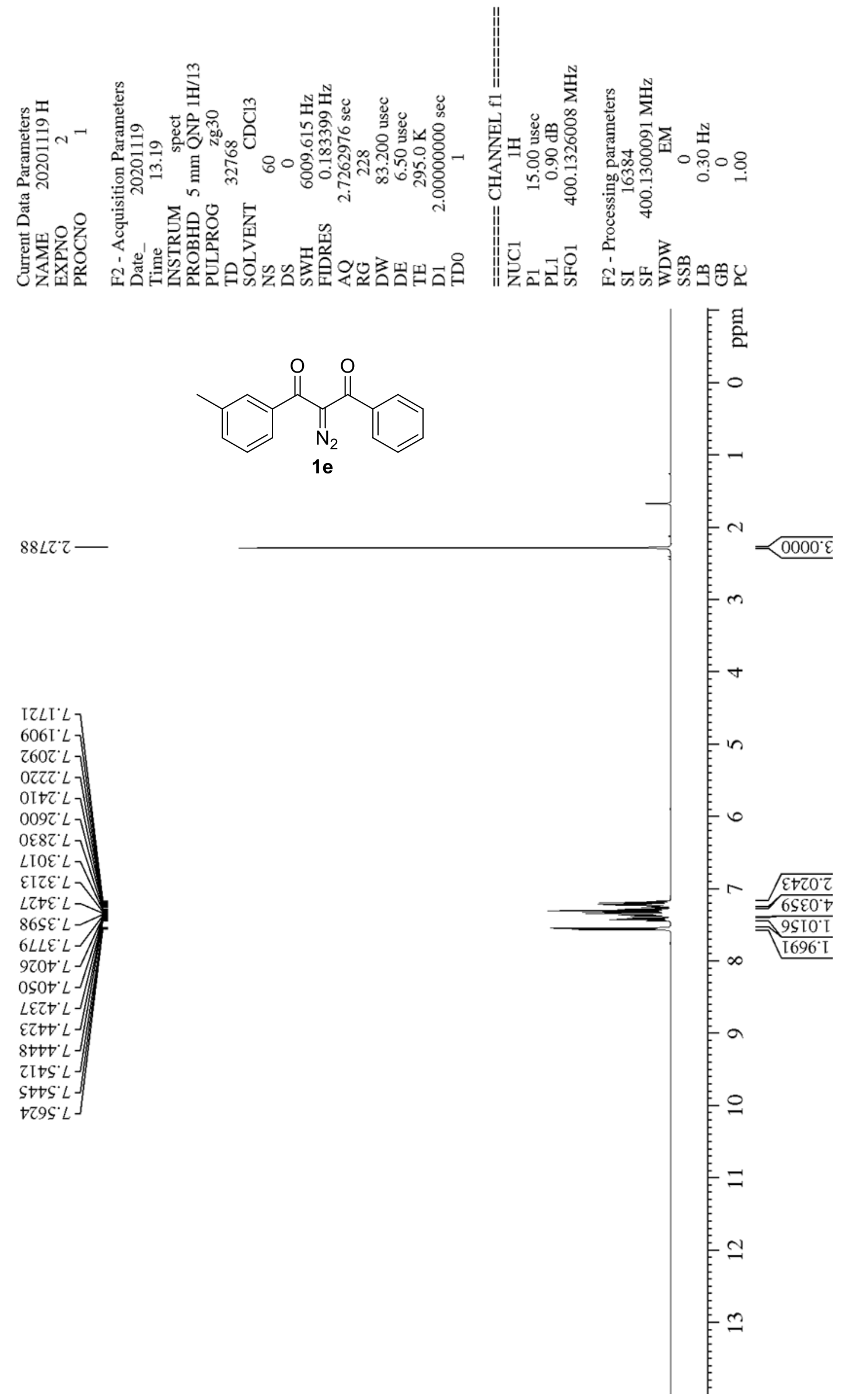




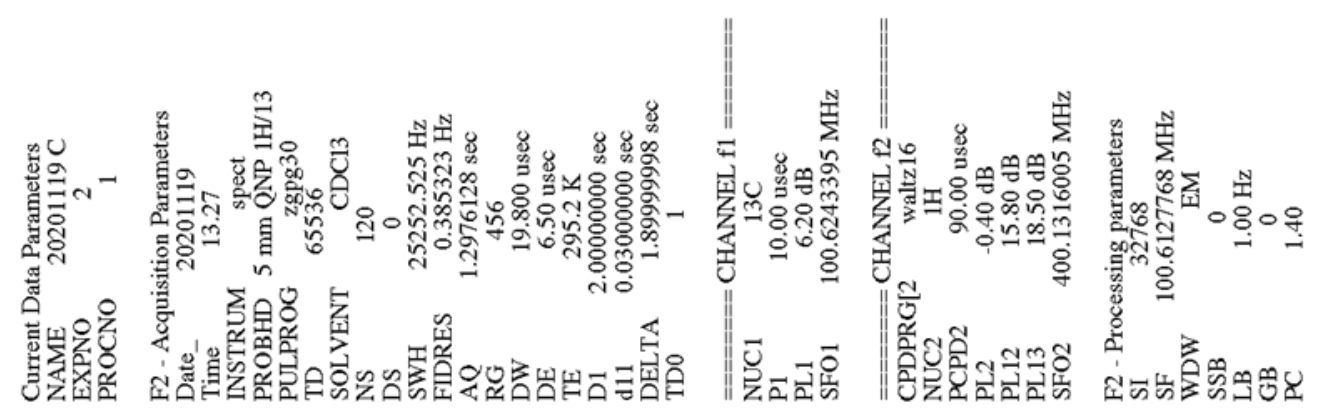

ESOI'IZ<smiles>Cc1cccc(C(=O)C(=N)C(=O)c2ccccc2)c1</smiles>

I $289^{\circ} 9 \mathrm{~L}$

$0000^{\circ} \mathrm{LL}$

SLIE'LL

stSC't8

$979 t^{\circ} \subseteq Z I$

$\angle 26 \mathrm{I}^{\circ} 8 \mathrm{II}$

\&I $\angle Z$ ' $8 Z \mathrm{II}$

0SE6 $8 Z \mathrm{ZI}$

ZSLT'ZEI

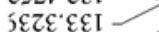

ZLE $9 \& \mathrm{I}$

OEI $0^{\circ} L E I$

ZZ9I' $8 \varepsilon$ I

$\operatorname{E60S}^{\prime} 98 \mathrm{I} \longrightarrow$

0ะZ9.98I $>$

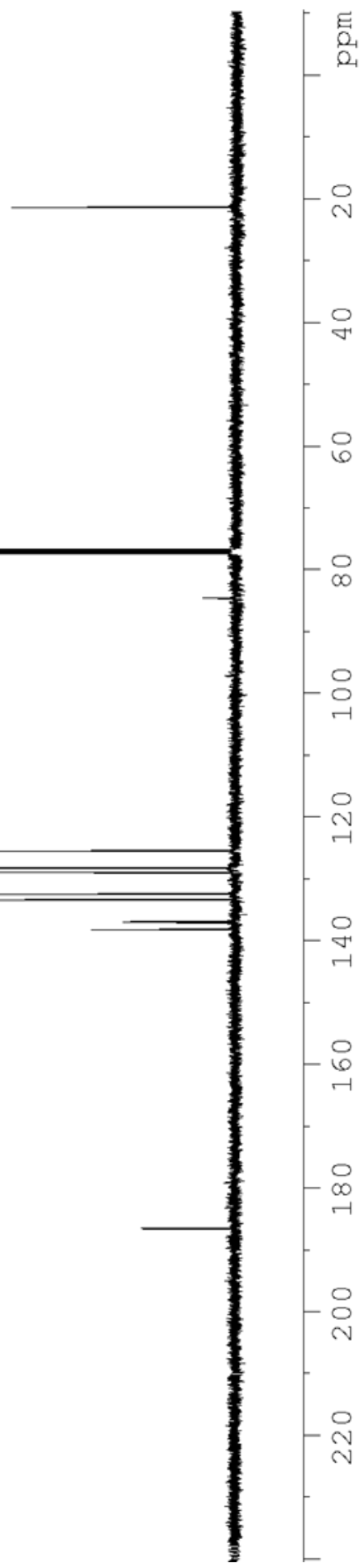



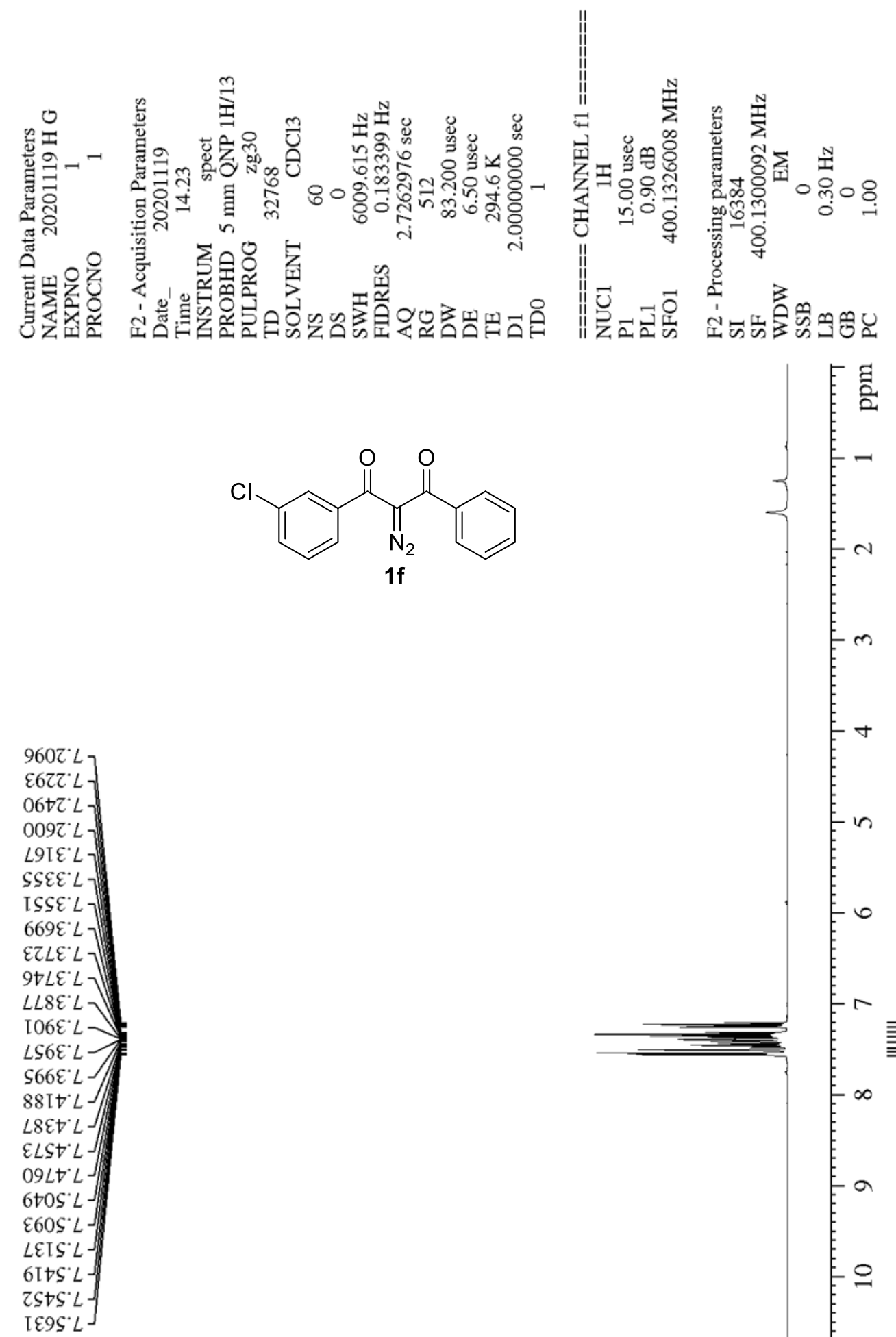<smiles>O=C(C(=[V])C(=O)c1cccc(Cl)c1)c1ccccc1</smiles>

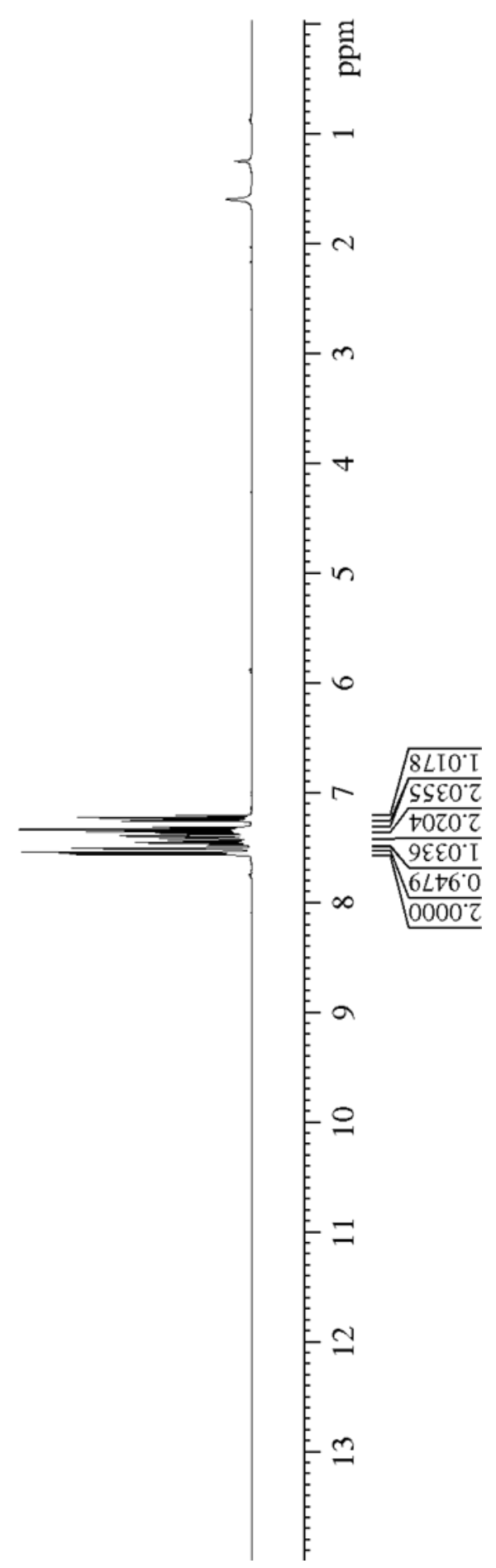



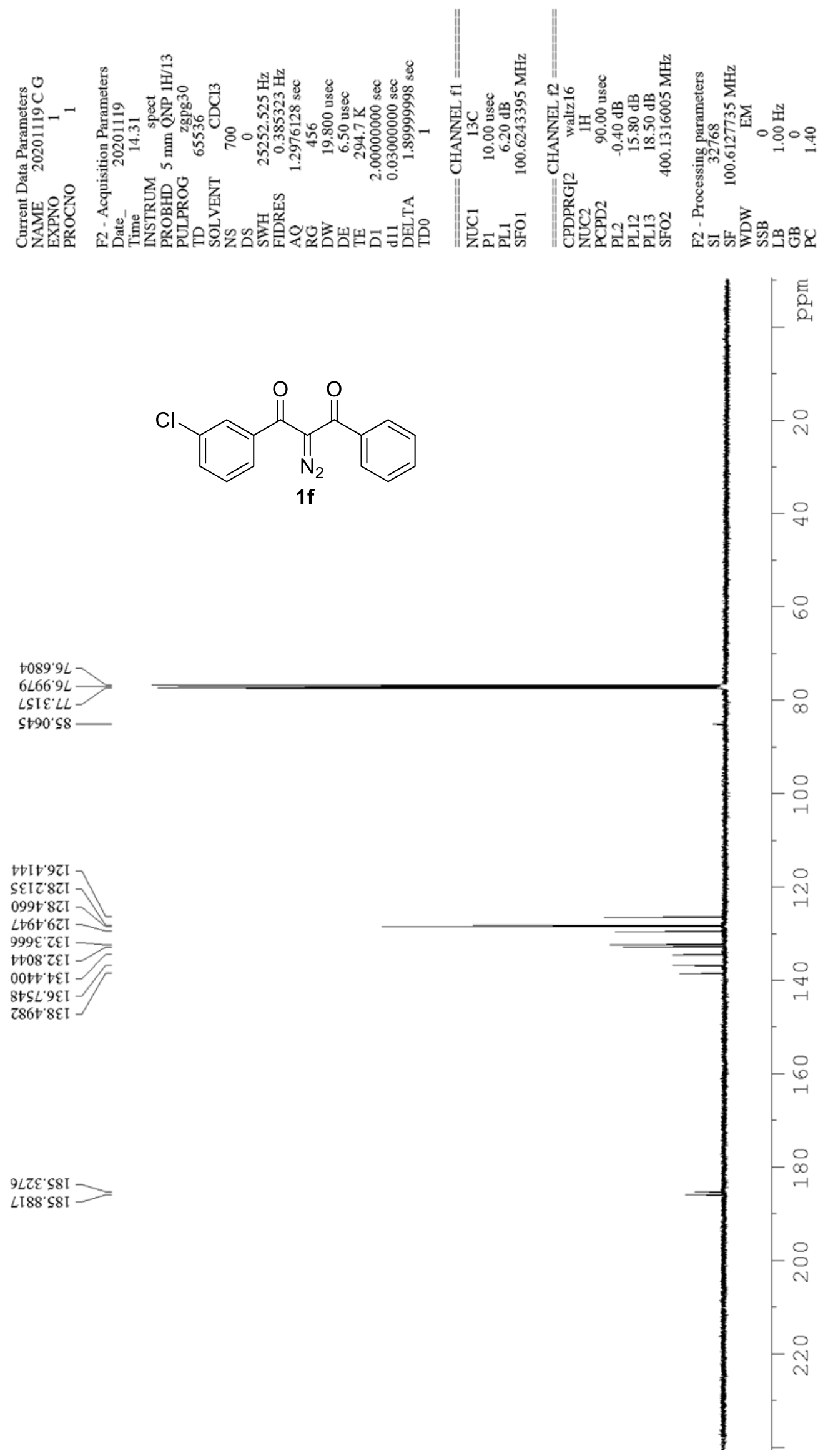

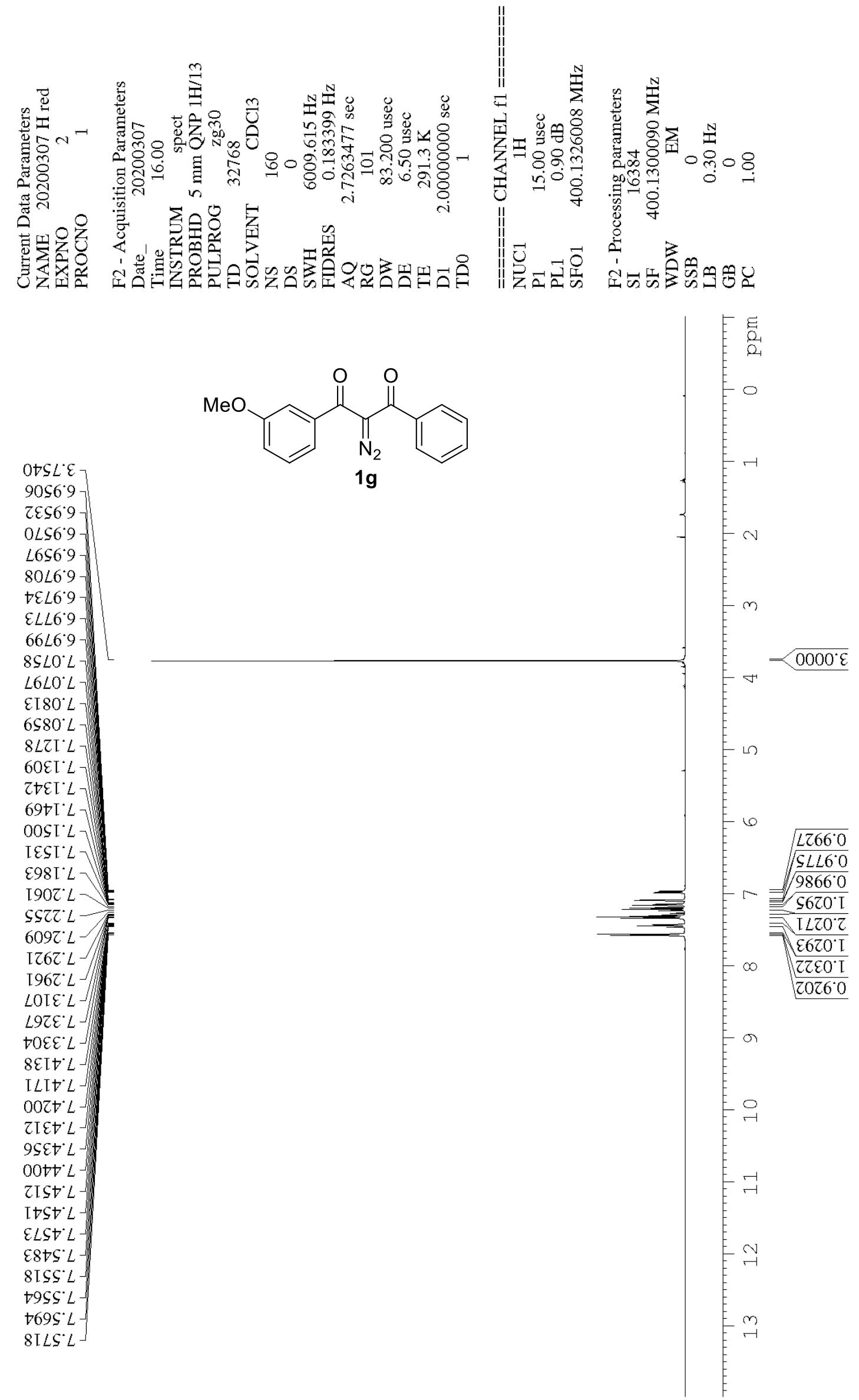


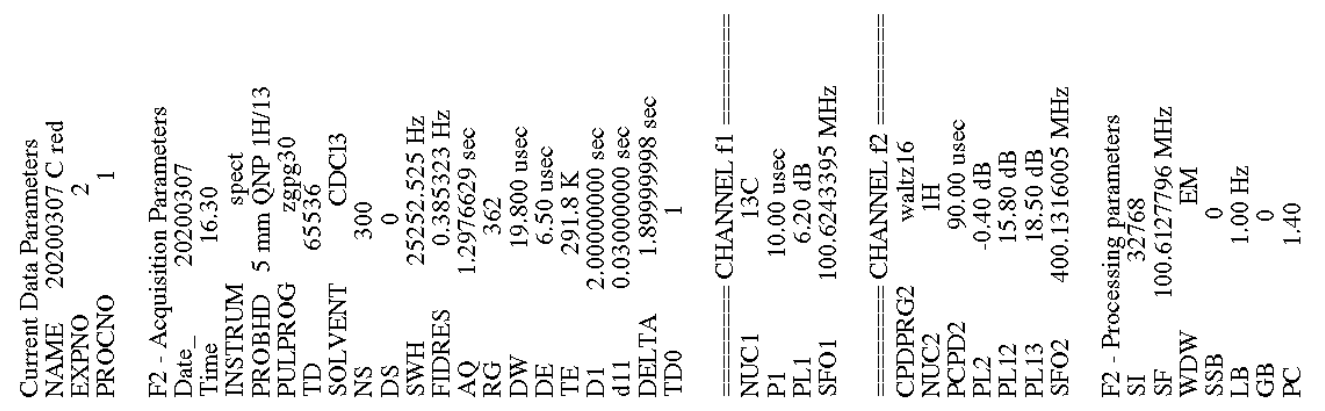

5II $\mathcal{S} \subseteq$<smiles>COc1cccc(C(=O)C(=[N+]=[N-])C(=O)c2ccccc2)c1</smiles>

$\$ \$ 89^{\circ} 9 L$

¿EOO $\mathrm{LL}$

SIZE $L L-$

$89 t t^{\circ}+8$

SLZ8'ZI I

9096 8I I

$09 Z L^{\circ} 0 Z I$

0 LOZ'8ZI

Z†8Z:8ZI

ZLIE'6ZI -

$00 t S^{\prime} Z \varepsilon I-$

OSI6. $9 E \mathrm{E}-$

SL80'8EI -

$998 \mathcal{E}^{\circ} 6 \mathrm{SI}$

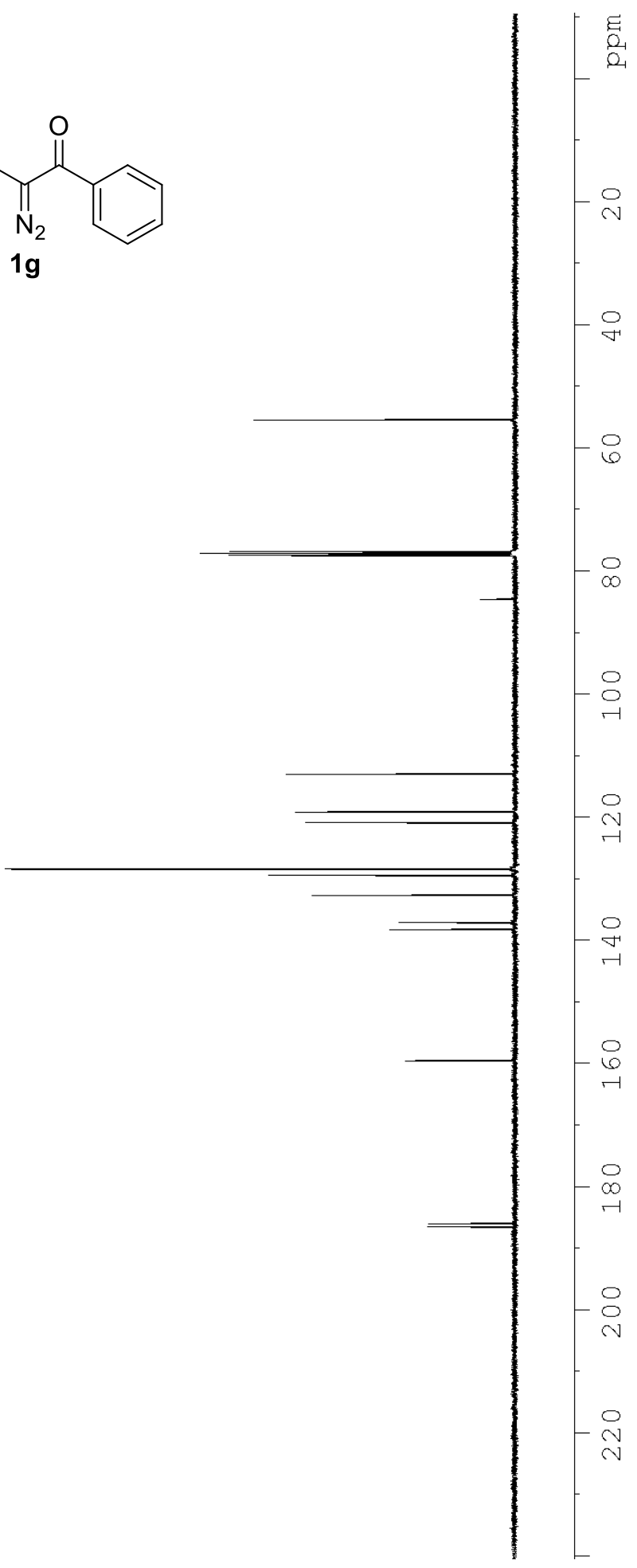

$9820^{\circ} 98 \mathrm{I}-$

LIIS'98I $>$ 

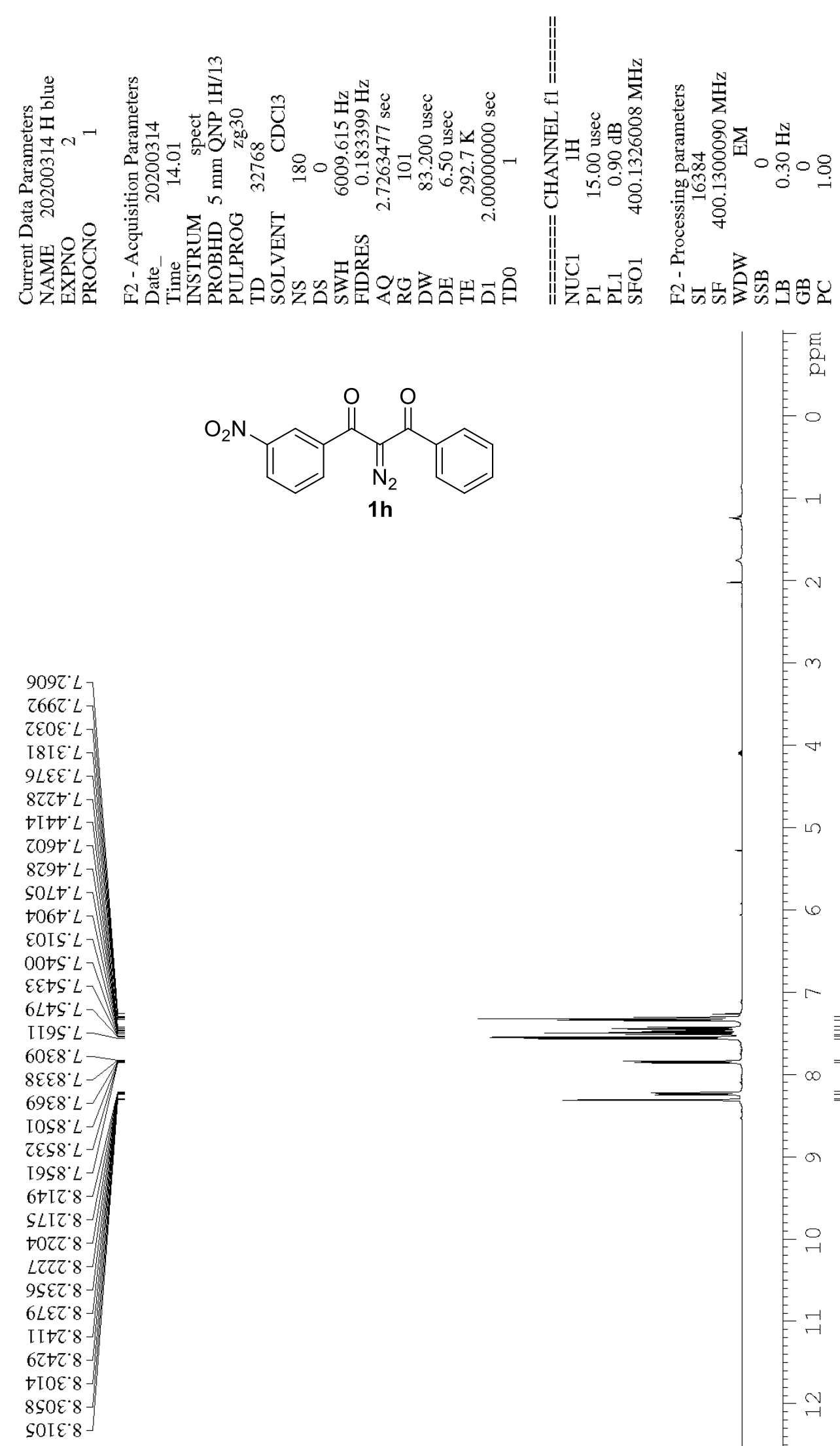<smiles>N=C(C(=O)C(=O)c1ccccc1)C(=O)c1cccc([N+](=O)[O-])c1</smiles>
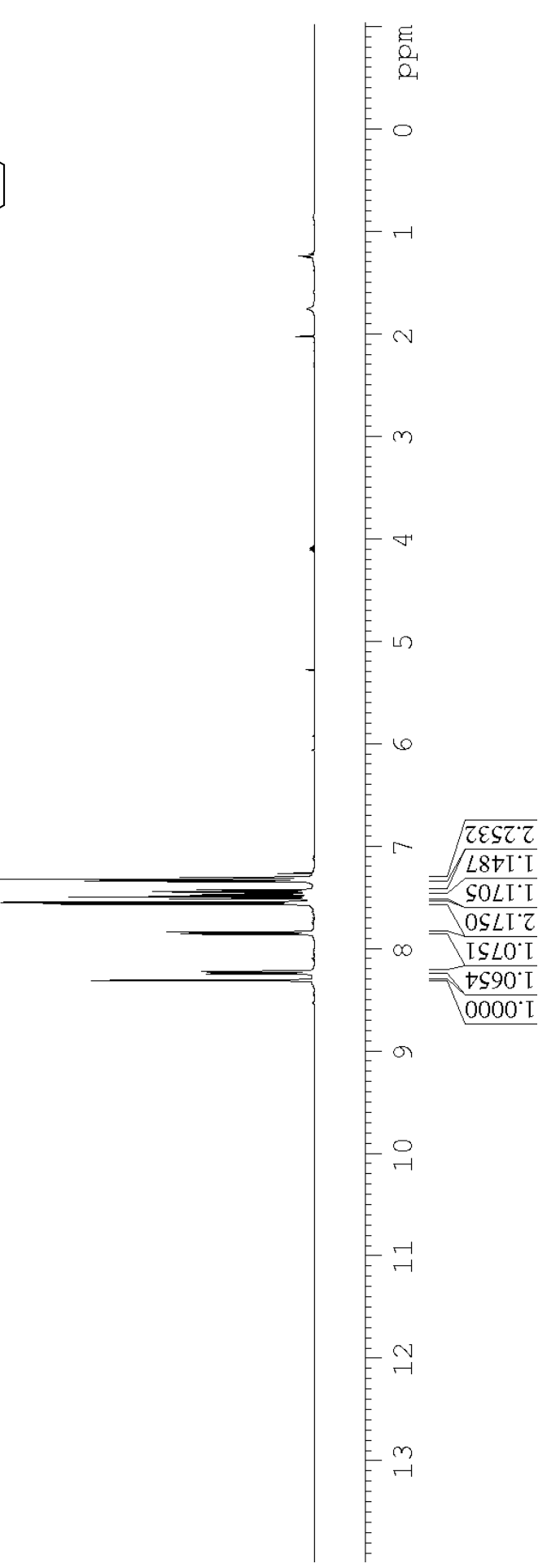

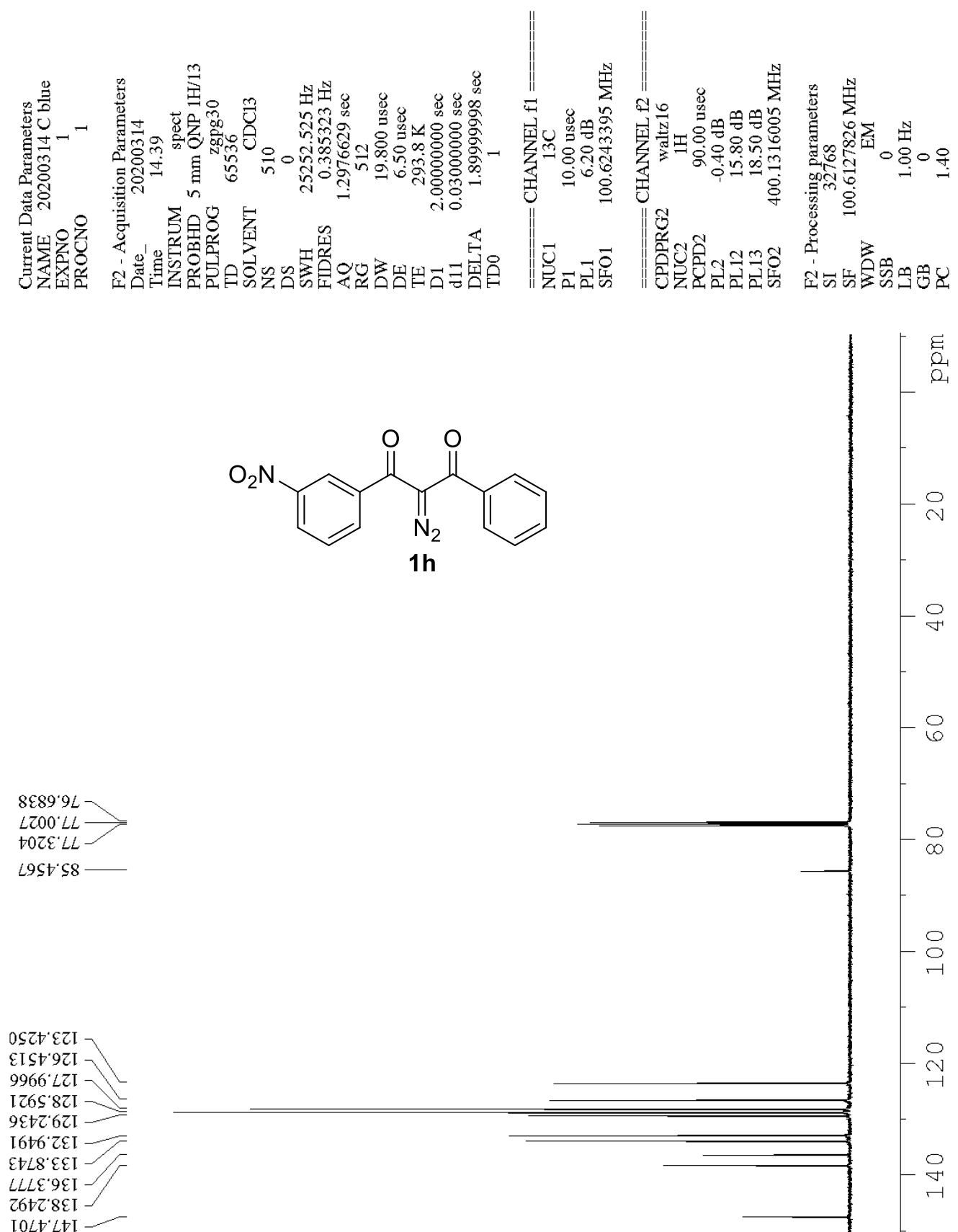<smiles>N=C(C(=O)C(=O)c1ccccc1)C(=O)c1cccc([N+](=O)[O-])c1</smiles>

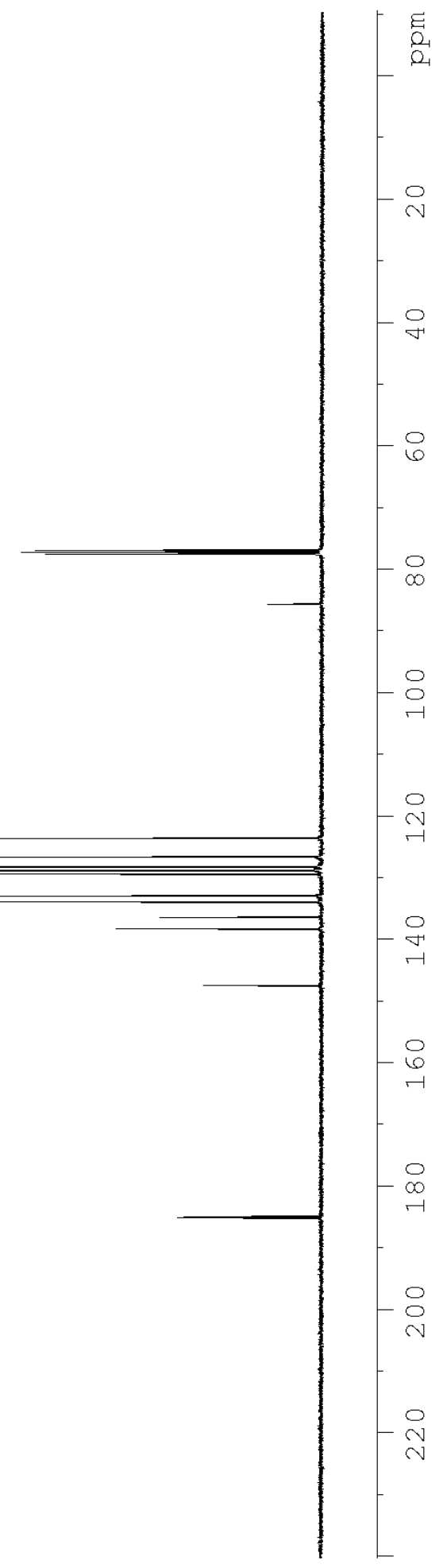

$\angle 08 L^{\circ}+8 \mathrm{I} \longrightarrow$ E8I0 $58 \mathrm{I}>$ 


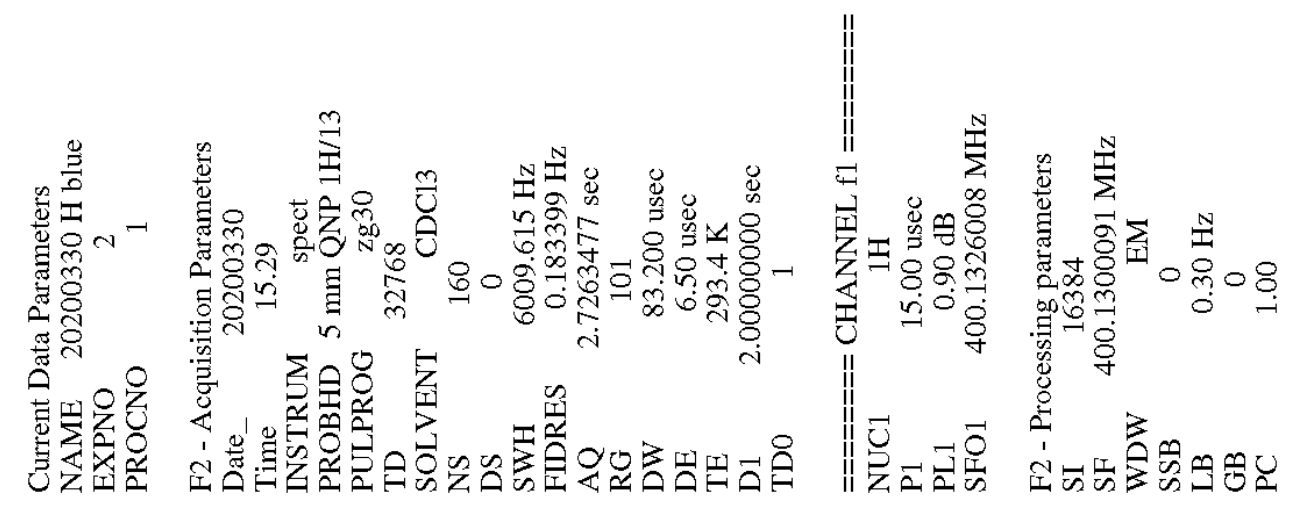<smiles>O=C(C(=O)c1ccccc1)C(=Nc1ccccc1)C(=O)c1ccc(-c2ccccc2)cc1</smiles>

$2092^{\circ} \mathrm{L}$

ZSIE L

I $₫ \varepsilon \varepsilon \cdot L$

$\subseteq E S E L$

OELE:L

$6+8 \varepsilon<-$

ZT6E $L$ -

$\angle 96 \varepsilon^{\circ} L$

[60t' $L$

8LZT L $L$

SOEt $L$

29t5 $L$

ESST $L$

$\angle D 9 t: L-$

I89t: $L$

OELt $L$ -

$\left.S L Z S^{\circ} L\right]$

$\Delta+t S \cdot L$

I $8+S^{\circ} L$

$079 S^{\circ} L$

ES9S $L$

SI09: L

$8619.2-$

OIS9 $L$

9[ $\angle 9^{\circ} L$

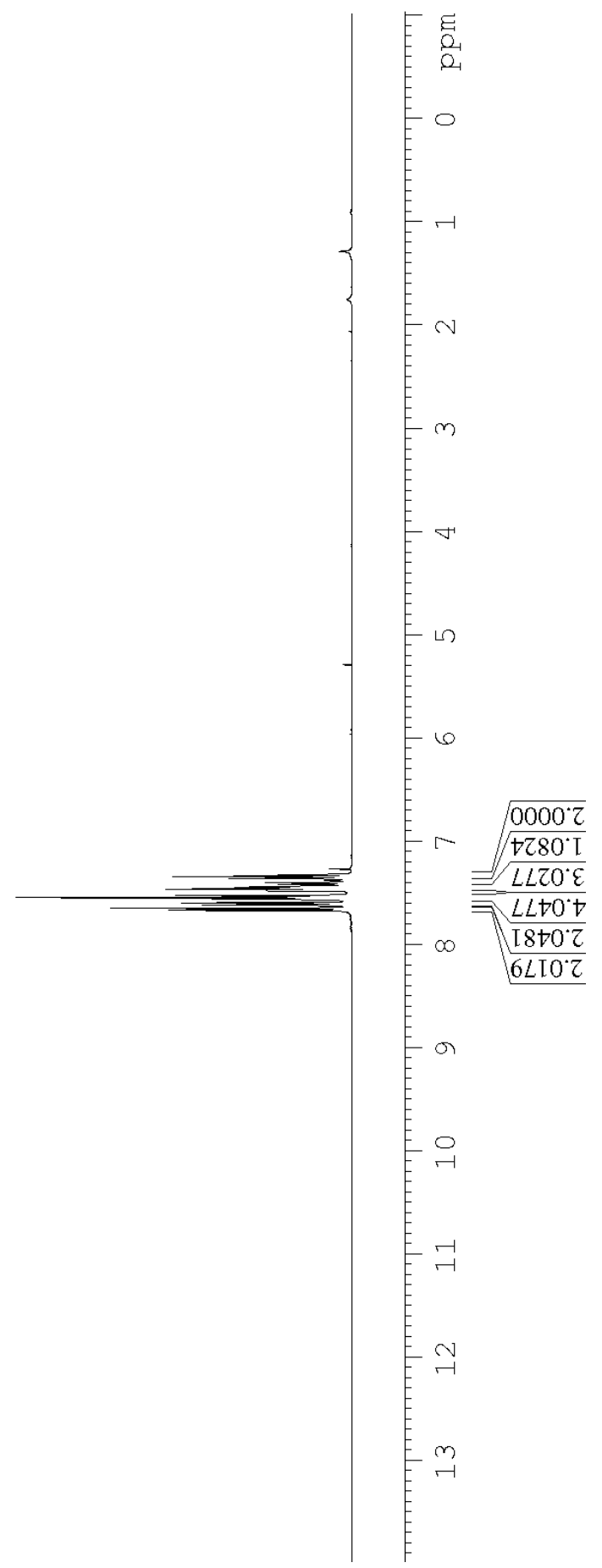




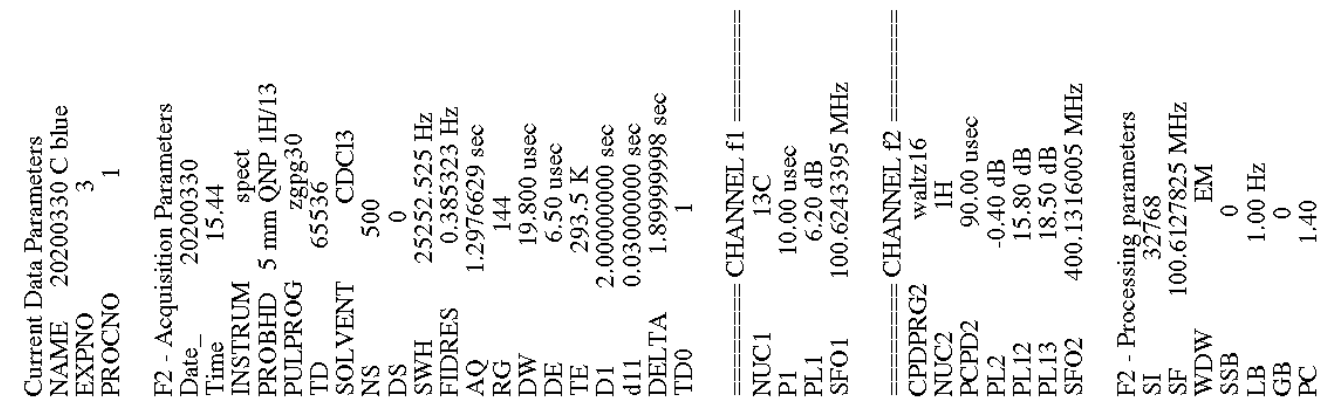

$0 \$ 89^{\circ} 9 L$

$\subseteq \mathcal{Q} 00^{\circ} \mathrm{LL}$

OIZE $L L$

6I0 ' 58<smiles>O=C(C(=O)c1ccccc1)C(=Nc1ccccc1)C(=O)c1ccc(-c2ccccc2)cc1</smiles>

$00 \varepsilon 8.9 Z \mathrm{I}$

SOZI'LZI

LISI'8ZI

998Z'8ZI -

$66 \mathrm{Z}^{\circ} 8 \mathrm{ZI}$

$5 \mathrm{t} 8^{\circ} 8 \mathrm{ZI}$

$0+86^{\circ} 8 \mathrm{ZI}$

†OES'ZEI

$\nabla L O S^{\circ} \subseteq \mathcal{I}$

IS68.9\&I

EZZ9"6EI

$\angle \downarrow 8 Z^{\prime} \varsigma \mathrm{I}$

$89 \mathrm{I} 6^{\circ} \mathrm{S} 8 \mathrm{I}-$

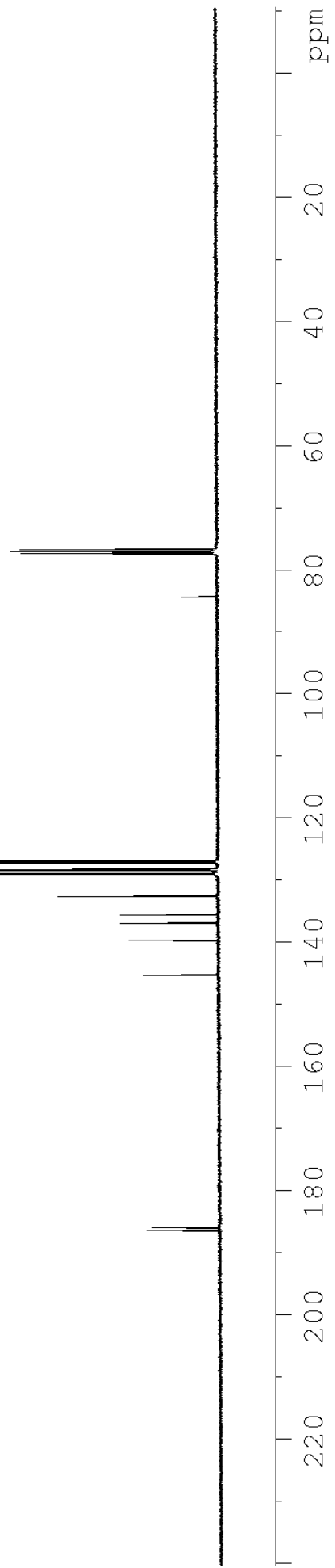




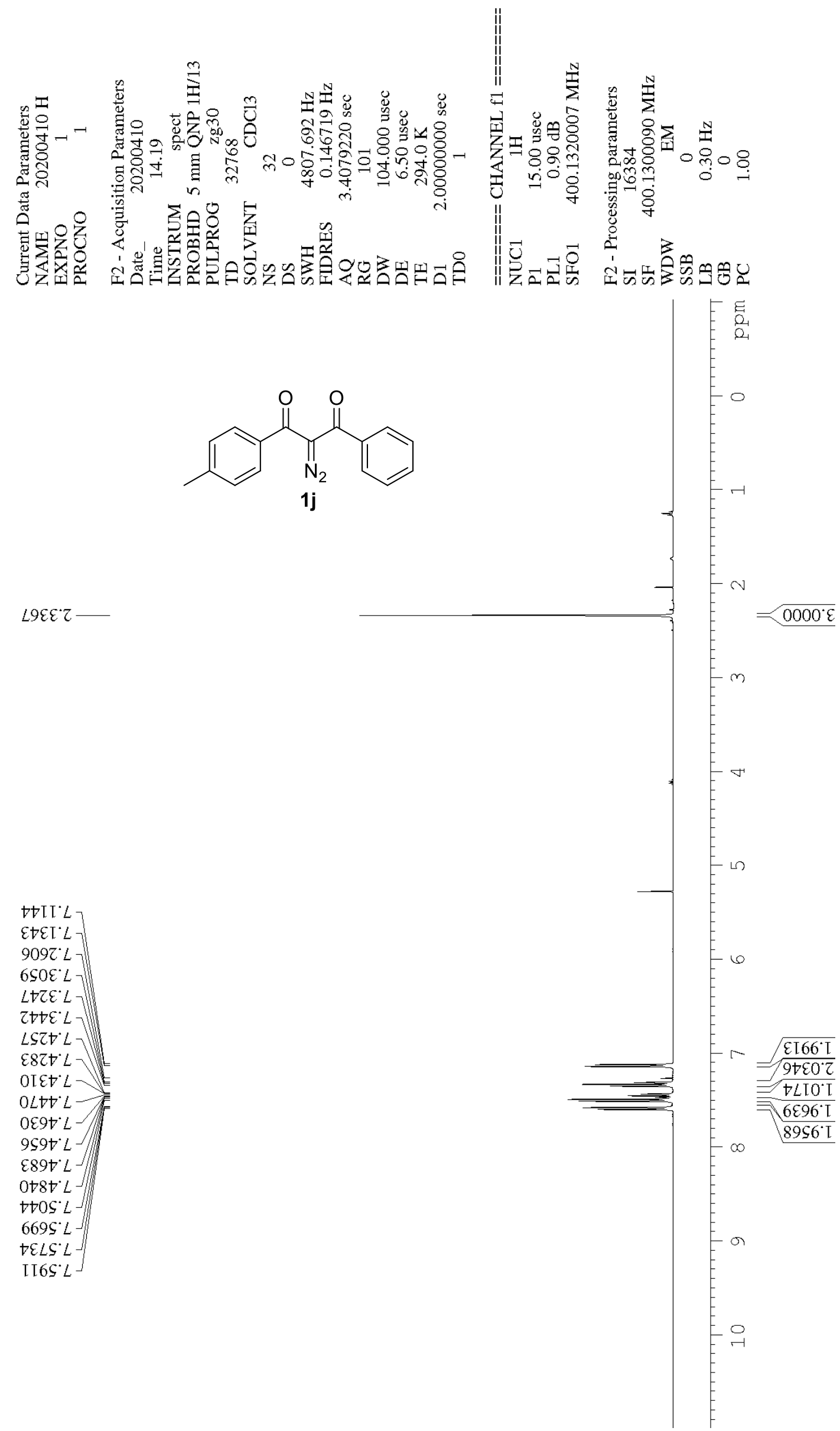




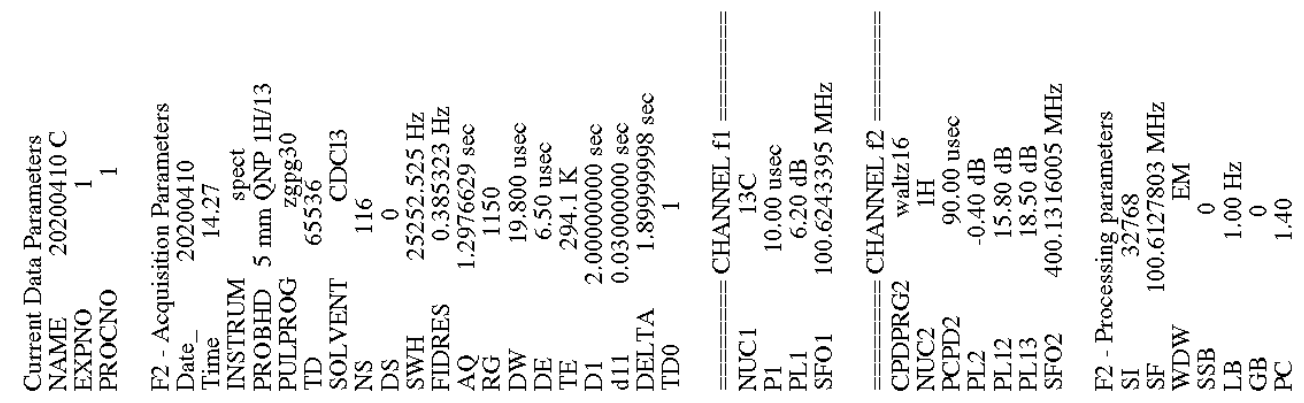

$I Z t s \cdot I Z$<smiles>Cc1ccc(C(=O)C(=N)C(=O)c2ccccc2)cc1</smiles>

1j

I $289^{\circ} 9 \mathrm{~L}$

$2000 . \mathrm{LL} \longrightarrow$

I8I $\mathcal{L} L L$

I069 $\mathcal{~} 8$

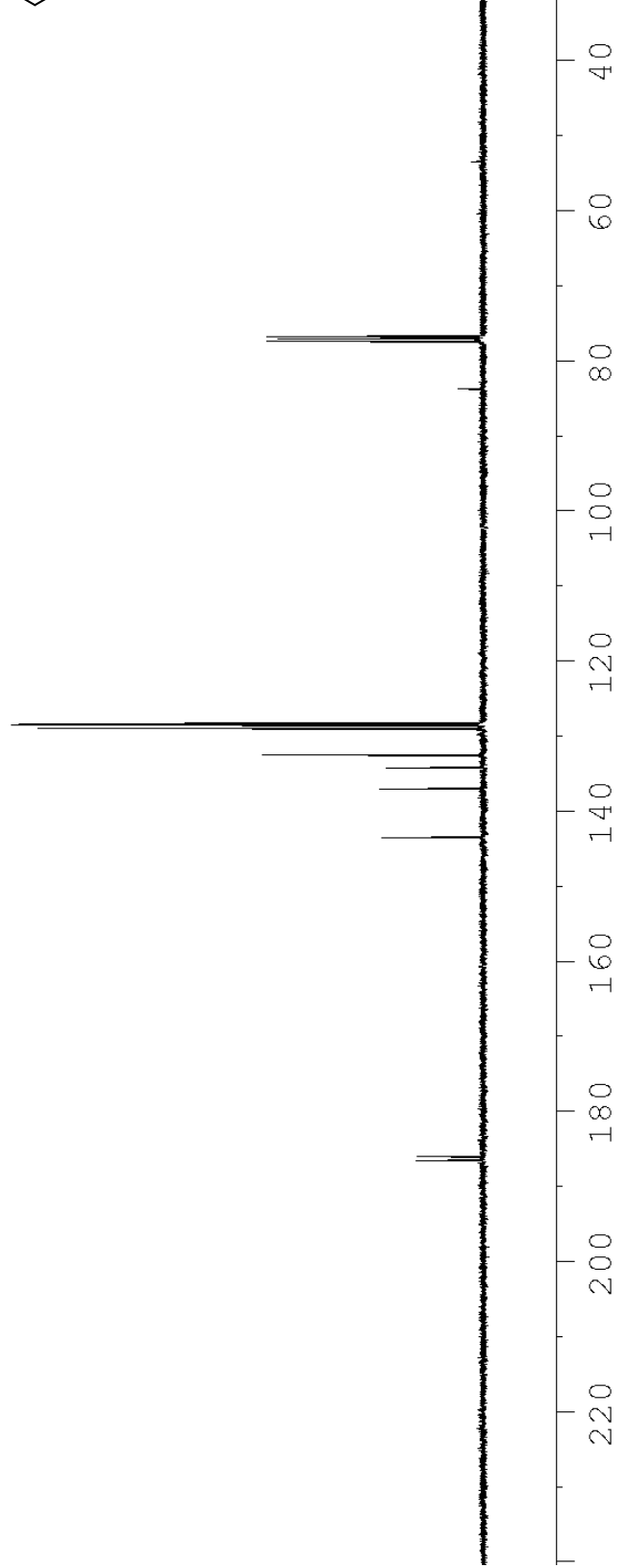

$|-| \begin{aligned} & E \\ & \tilde{a} \\ & -\end{aligned}$

$Z t \varepsilon Z \cdot 8 Z \mathrm{I}$

IE6Z'8ZI

\9Lt'8ZI

$89 \varepsilon 6.82 \mathrm{I}$

I $\angle 9)^{\prime} Z \varepsilon \mathrm{I}$

$\nabla L E I{ }^{\circ} \downarrow \varepsilon \mathrm{I}$

I $\angle t 6^{\circ} 9 \varepsilon \mathrm{I}$

tI $6 t^{\circ} \mathcal{E} t \mathrm{I}$

$2656.581-$

$68 \mathrm{IS} 98 \mathrm{I} \longrightarrow$ 


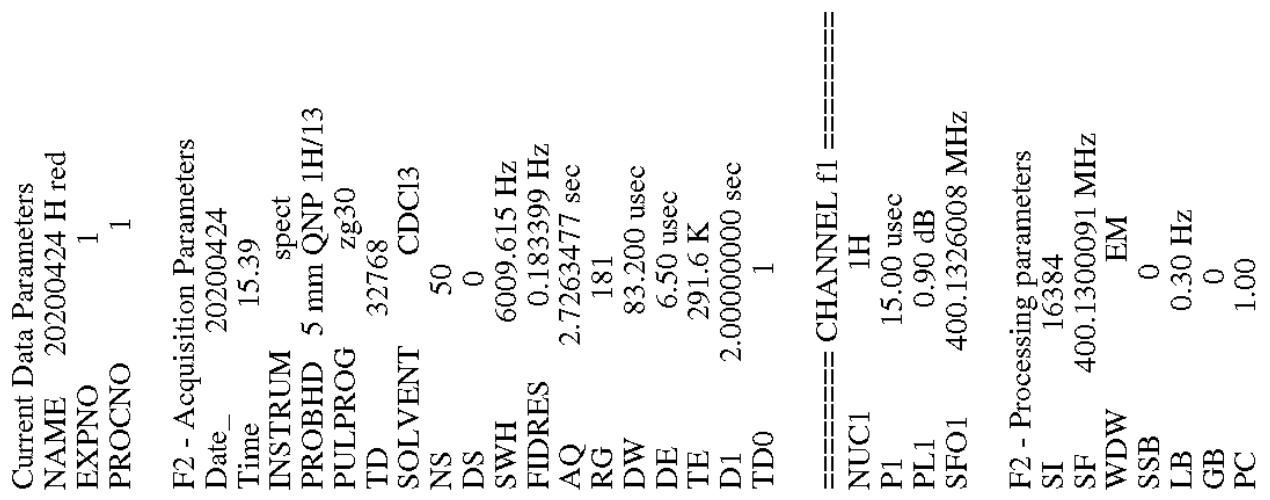<smiles>N=C(C(=O)C(=O)c1ccccc1)C(=O)c1ccc(Cl)cc1</smiles>
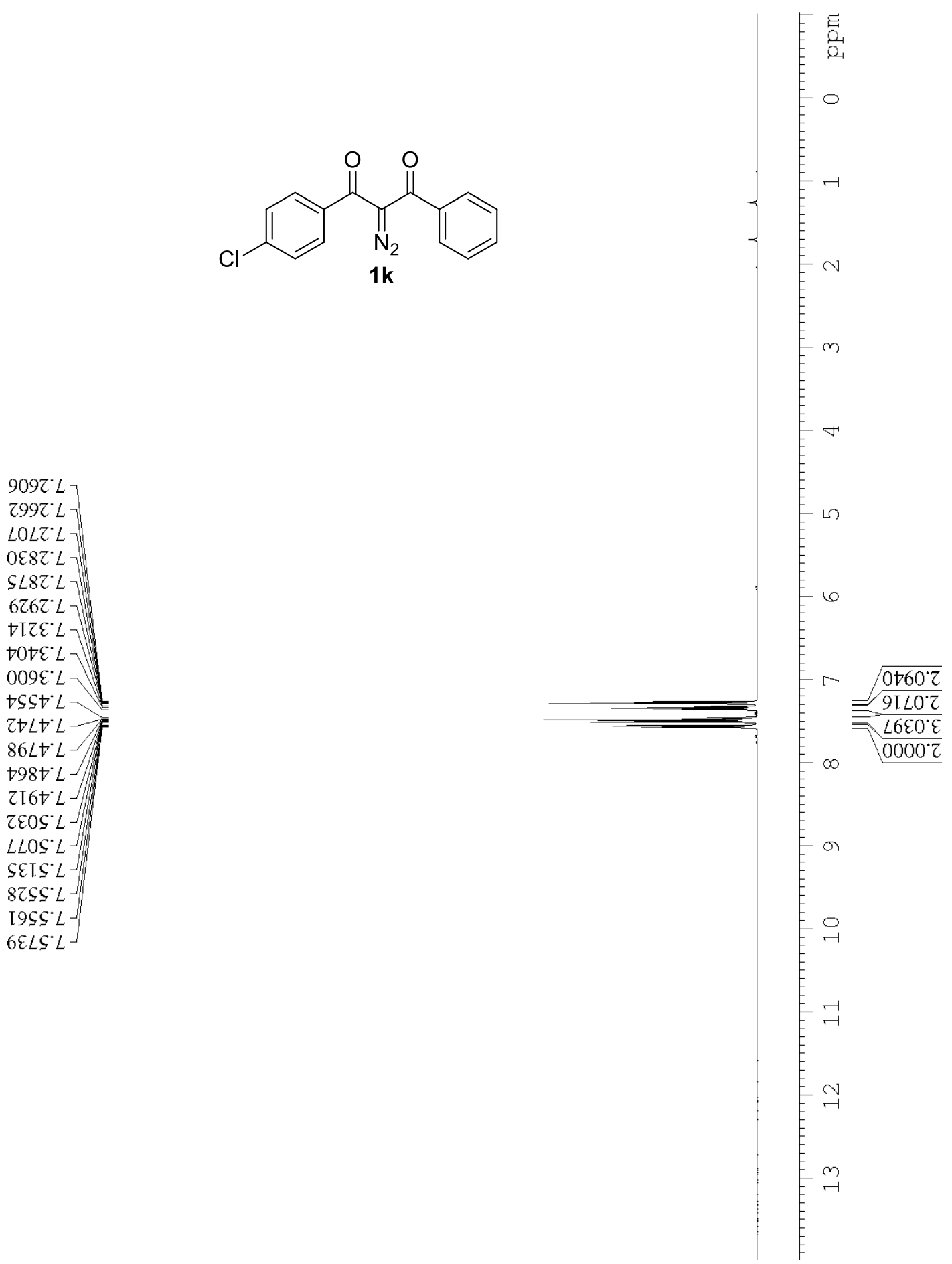


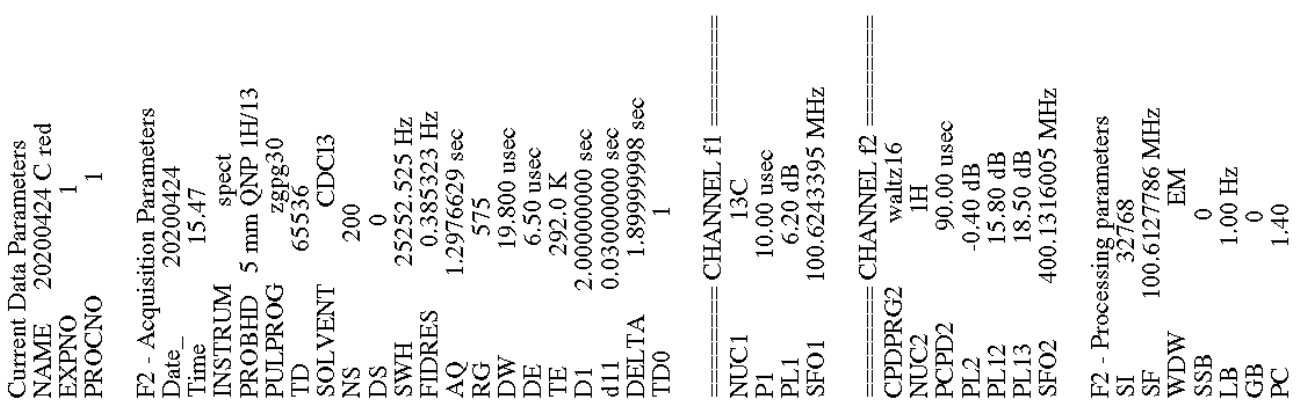

$0689^{\circ} 9 L$

$\varepsilon 900^{\circ} L L$

$8 \nabla Z E^{\prime} L L-$

$06 \angle t+8$

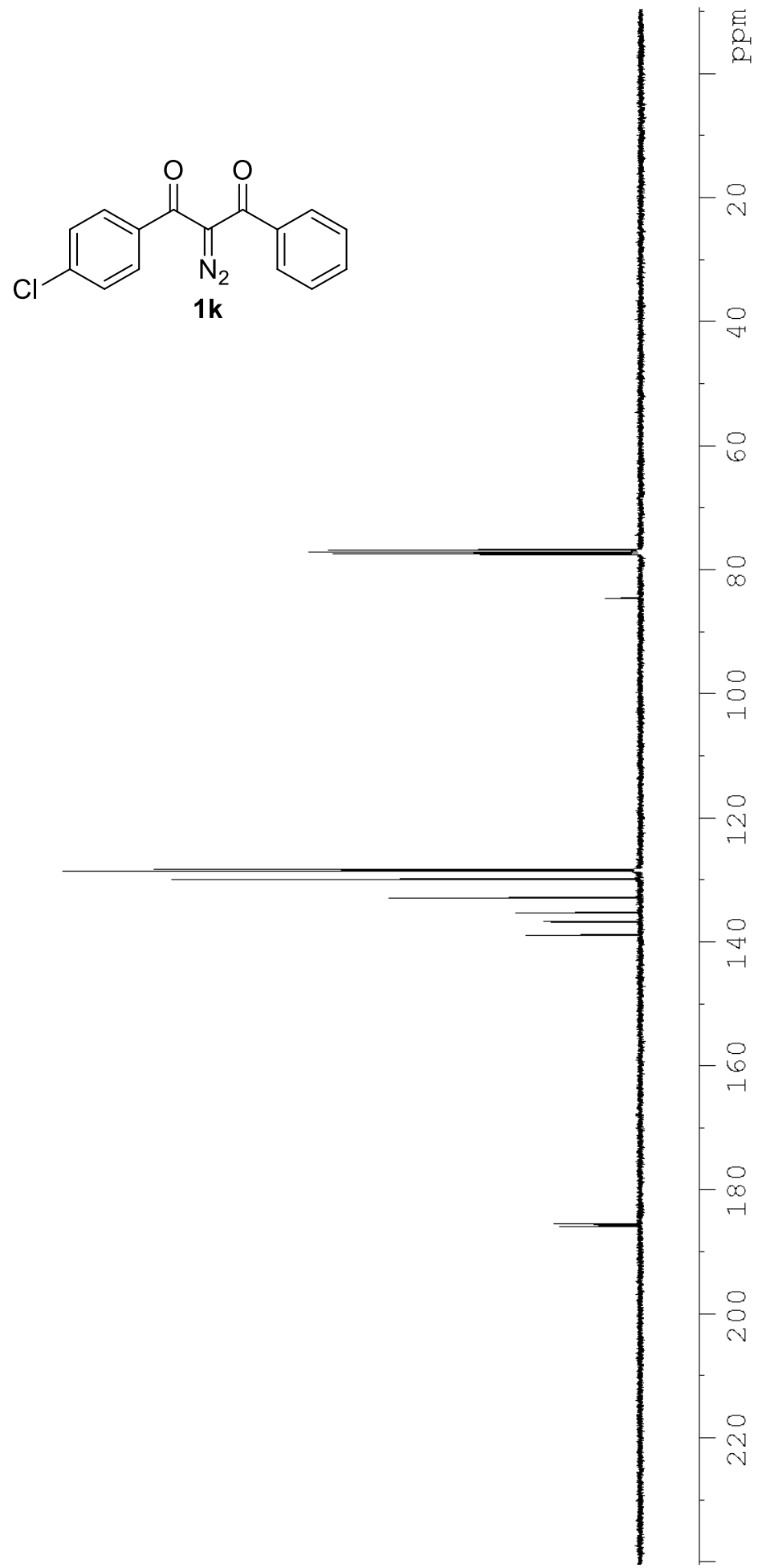

\$6IZ:8ZI

EZ9t.8ZI

$9587^{\circ} 8 \mathrm{ZI}$

$\varepsilon 908^{\circ} 6 \mathrm{Z}$

$9 \angle 08^{\circ} Z E \mathrm{I}$

$\angle \mathrm{IOZ} \subseteq \mathcal{} \subseteq \mathrm{I}$

$\varsigma \mathcal{E} \angle 9^{\circ} 9 \mathcal{E} \mathrm{I}$

$9608^{\circ} 8 \mathcal{E} \mathrm{I}$

sZtt $\$ 8 \mathrm{I}-$

†608. $\mathrm{S} 8 \mathrm{I}$ 

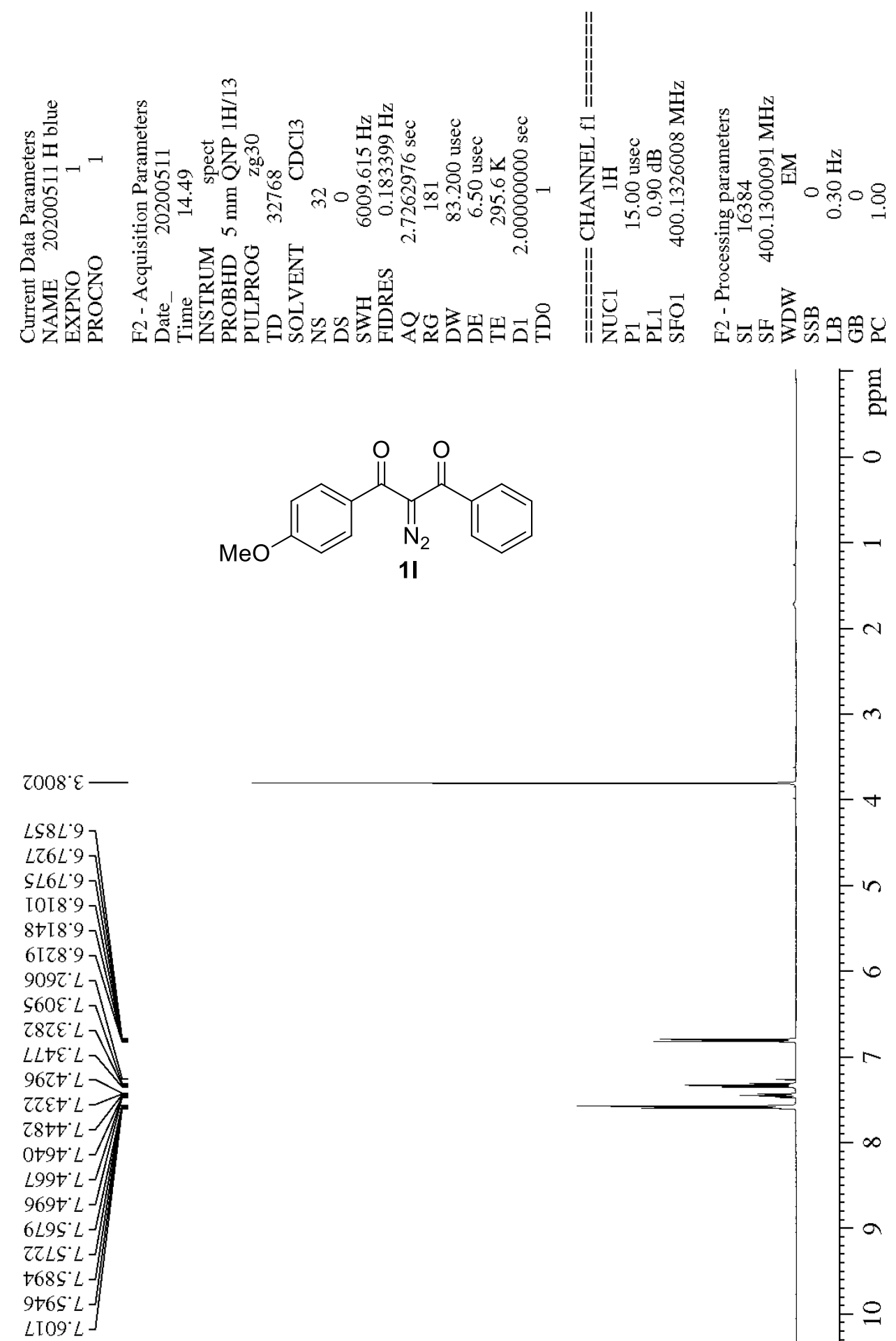<smiles>[CH]=[N+]=C(C(=O)c1ccccc1)C(=O)c1ccc(OC)cc1</smiles>

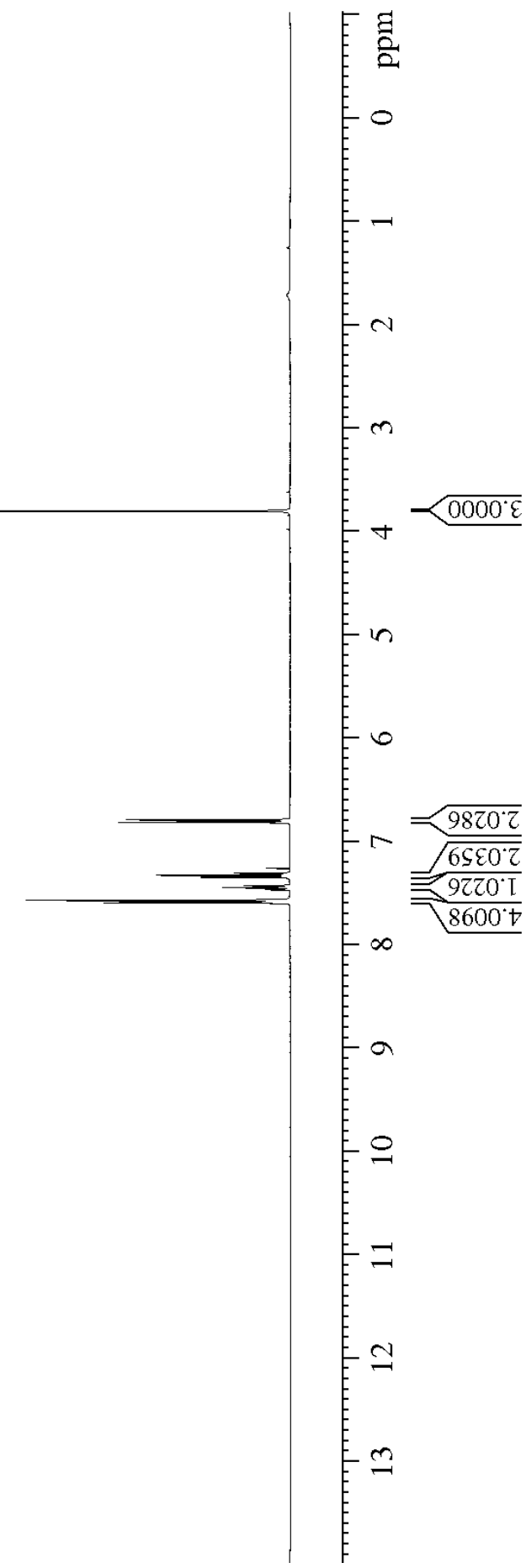




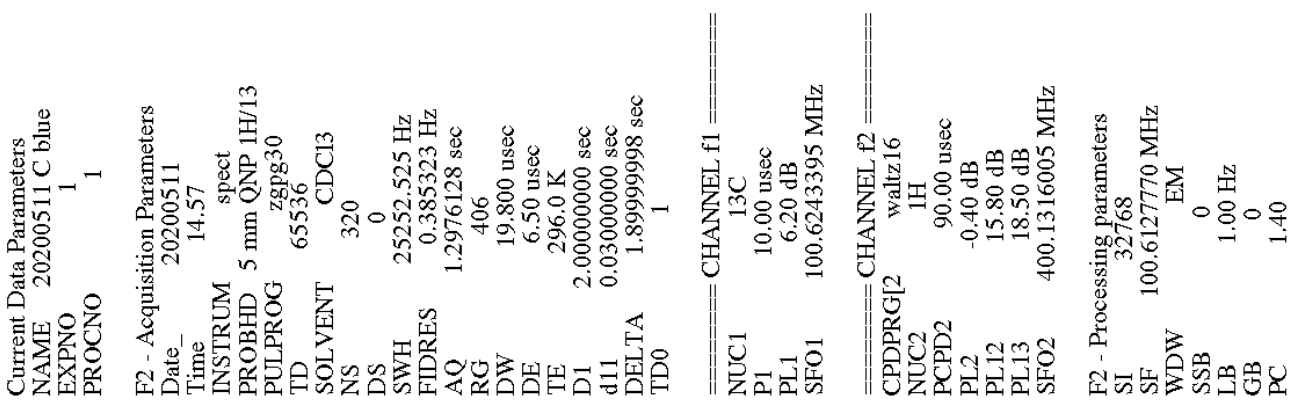<smiles>COc1ccc(C(=O)C(=N[Tl])C(=O)c2ccccc2)cc1</smiles>

$3 s_{00} \mathrm{~S} \varsigma$

$8+89^{\circ} 9 \mathrm{~L}$

$Z \varepsilon 00 \mathrm{LL} \longrightarrow$

SOZE $L L$

OOSt. $\mathcal{L S ~}_{-}$

8It $\mathcal{S}^{\prime} \varepsilon I I-$

$\angle 682.82 \mathrm{I}$

I00E' $8 \mathrm{ZI}$

ऽऽEع $6 Z \mathrm{I}$

68\&8.0 0 I

896t'ZEI

L6EO $L E I-$

$\mathcal{\varepsilon} \mathrm{I} Z^{\prime} \varepsilon 9 \mathrm{I}$

$8688^{\circ}+8 \mathrm{I}-$

$9+79^{\prime} 98 \mathrm{I}-$

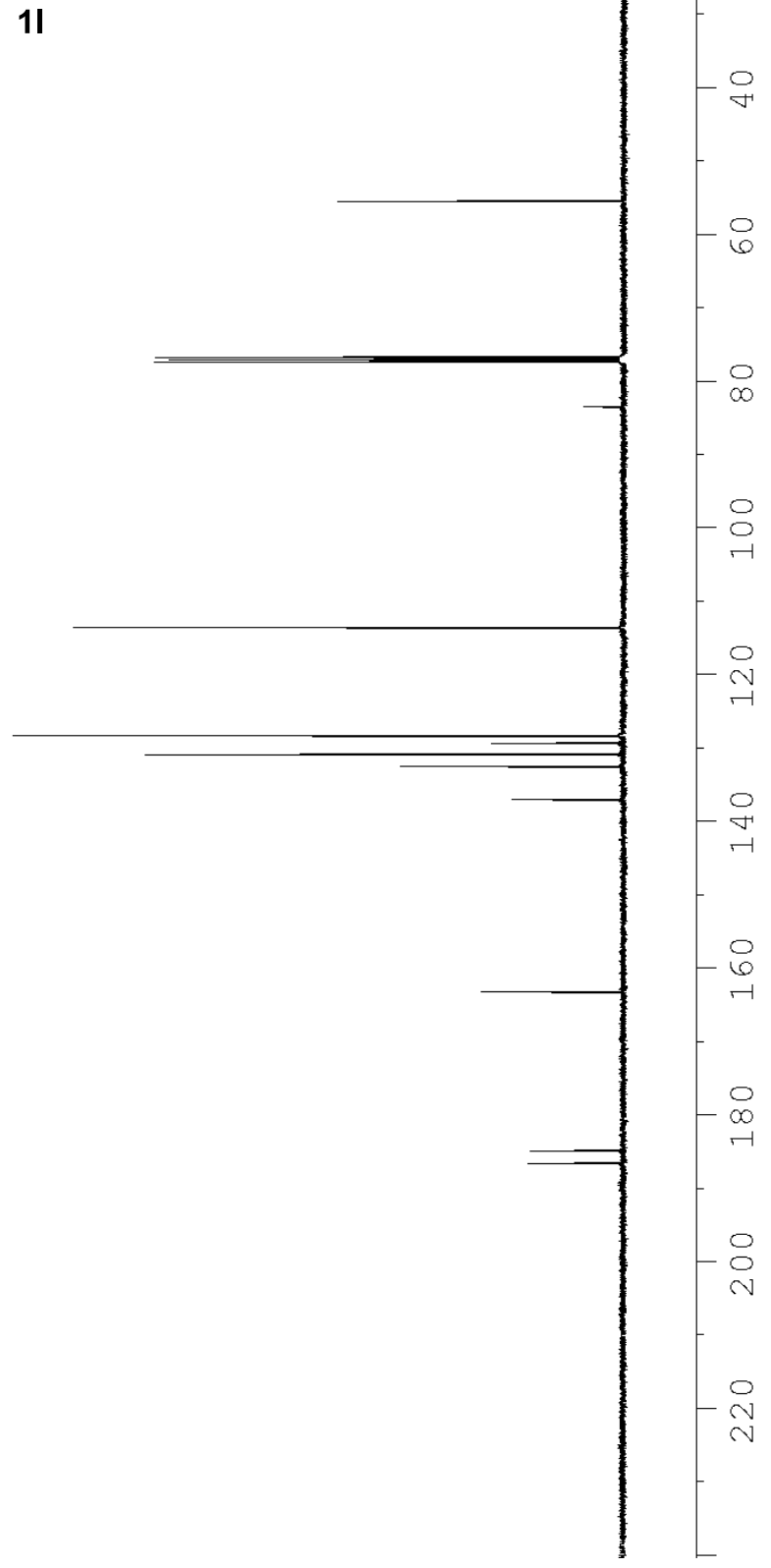



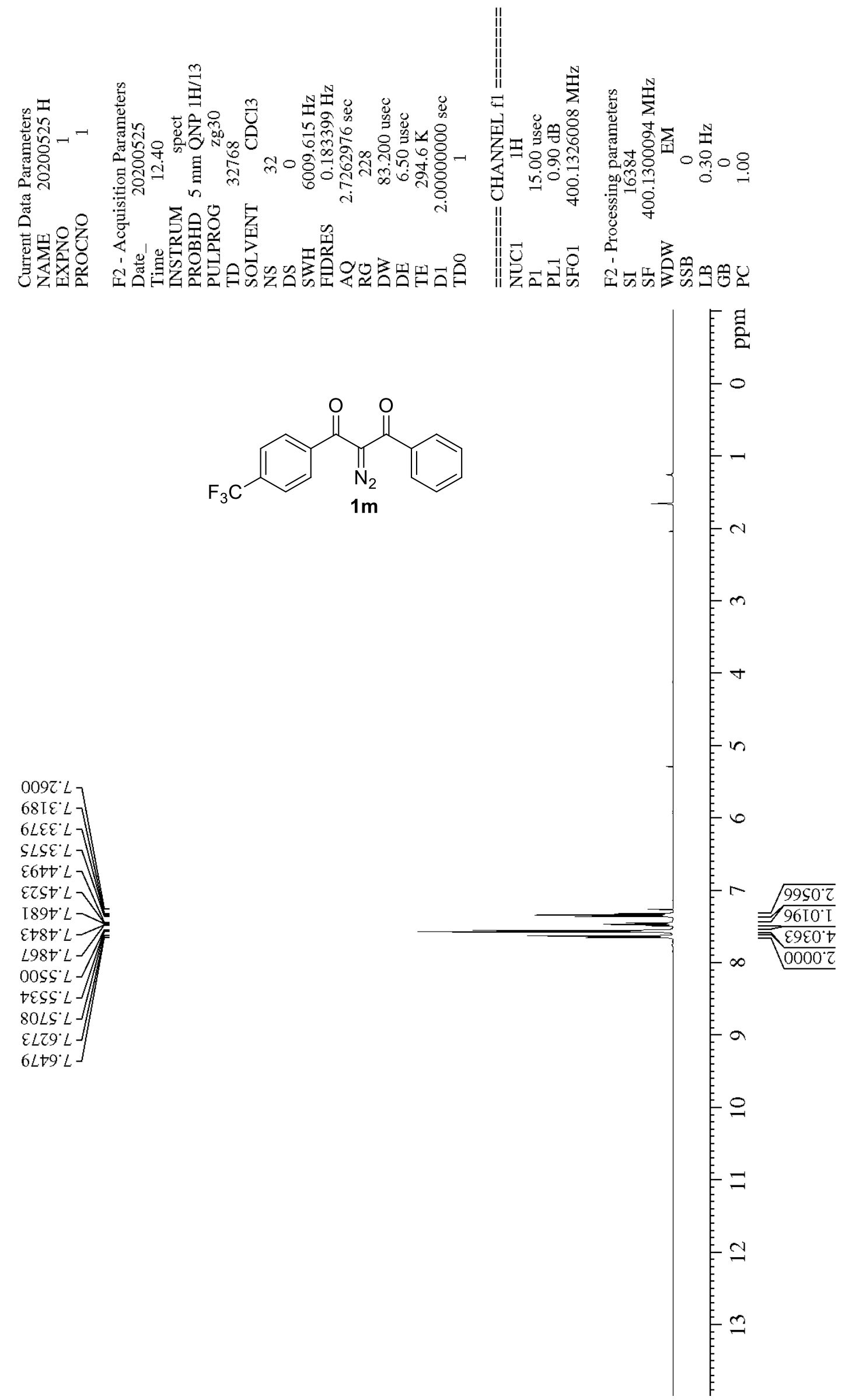<smiles>N=C(C(=O)C(=O)c1ccccc1)C(=O)c1ccc(C(F)(F)F)cc1</smiles> 


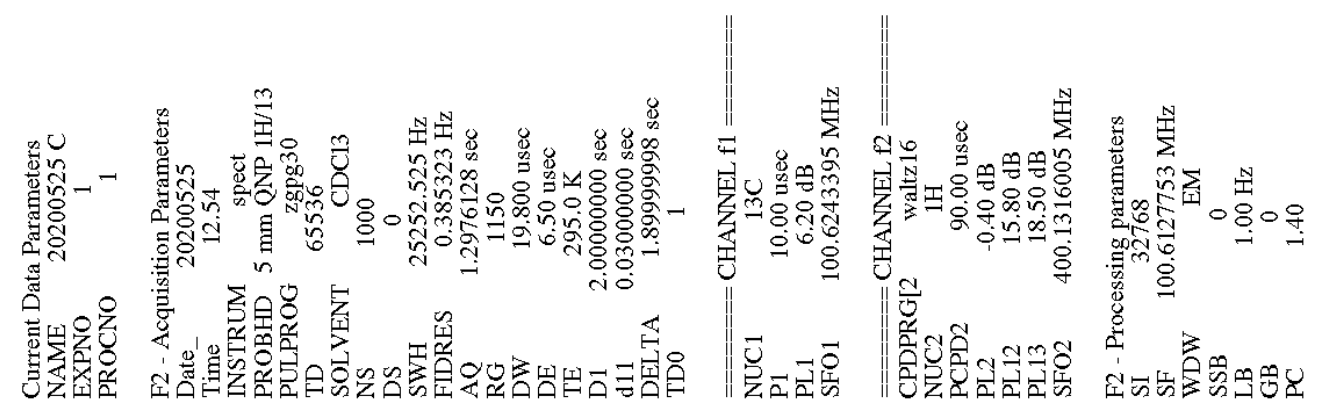

$\$ 289^{\circ} 9 L$

$0000^{\circ} \mathrm{LL}$

I 8 I $E L L$

$\varepsilon 890^{\circ} \varsigma 8$<smiles>N=C(C(=O)c1ccccc1)C(=O)c1ccc(C(F)(F)F)cc1</smiles>

EZoE'6I I

SZIO'ZZI

SZZL'†ZI

EEII'SZI

s6tI sCI

$8 \varepsilon Z Z$ ऽZI -

$\angle I E T^{\circ} L Z I$

ELSI' $8 Z I$

$96+S^{\prime} 8 Z \mathrm{I}-$

I8I9.8ZI

$\varepsilon\llcorner Z 6$ ZEI

LStI'EEI

I $L L t^{\circ} \varepsilon \varepsilon \mathrm{I}-$

I $08^{\circ} \varepsilon \varepsilon \mathrm{I}$

Z9ZI' $\bullet \varepsilon I$

эtSc' $9 \varepsilon \mathrm{I}$

$80 L 0^{\circ} 0 \mathrm{tI}$

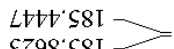

$1 \mathrm{~m}$

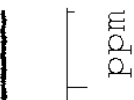

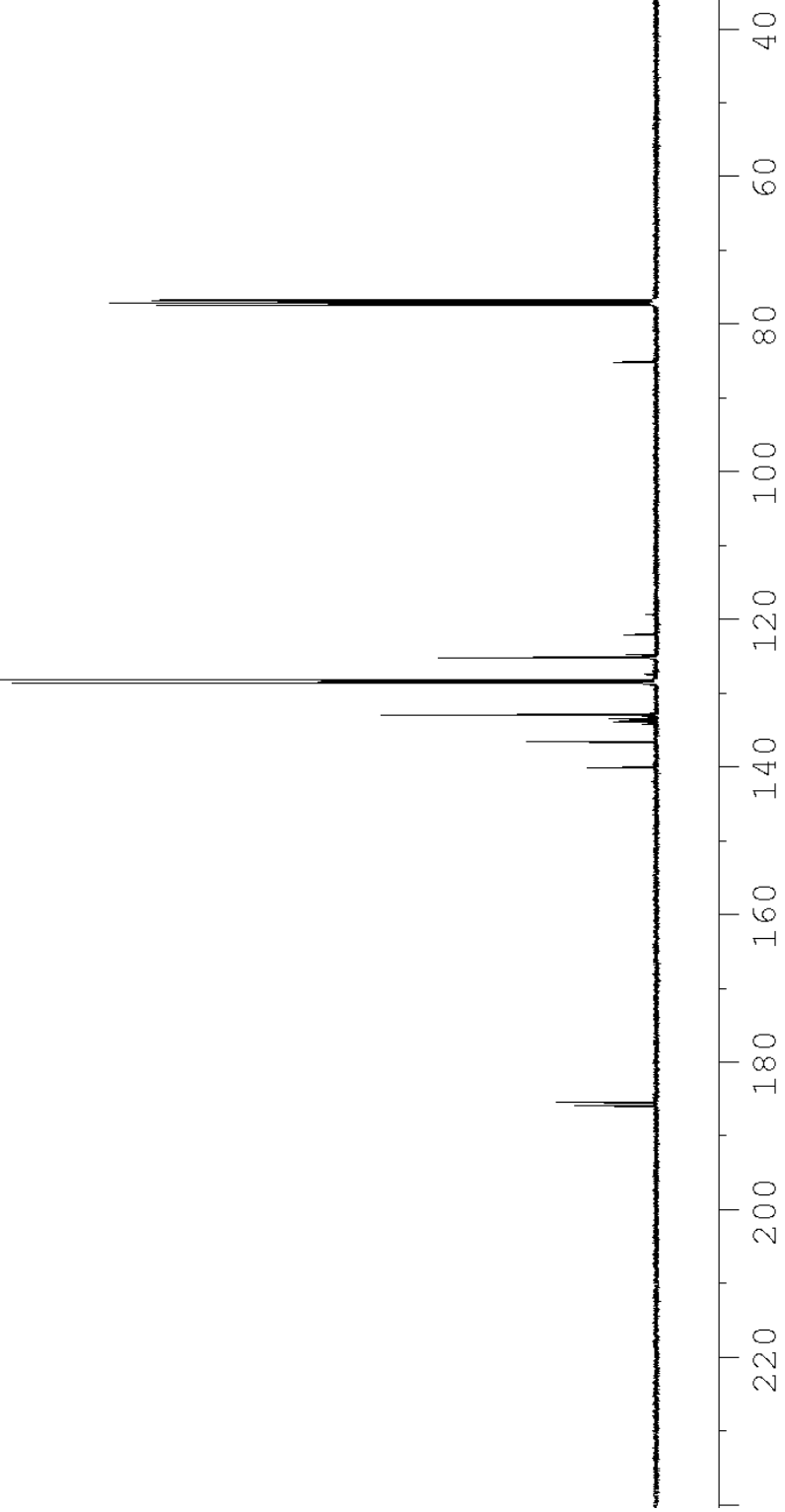




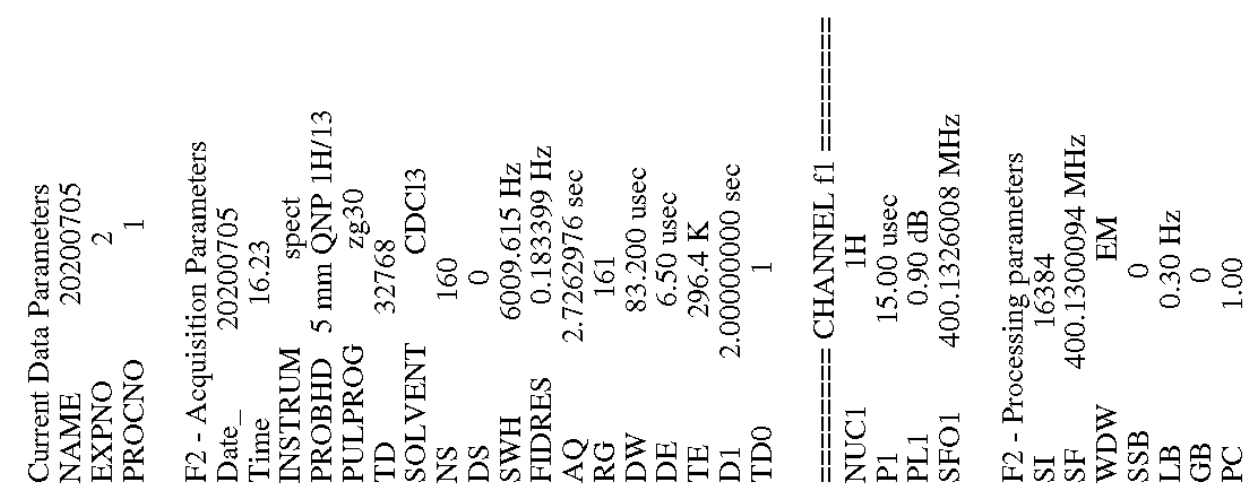<smiles>N=C(C(=O)c1ccccc1)C(=O)c1ccc2c(c1)OCO2</smiles>
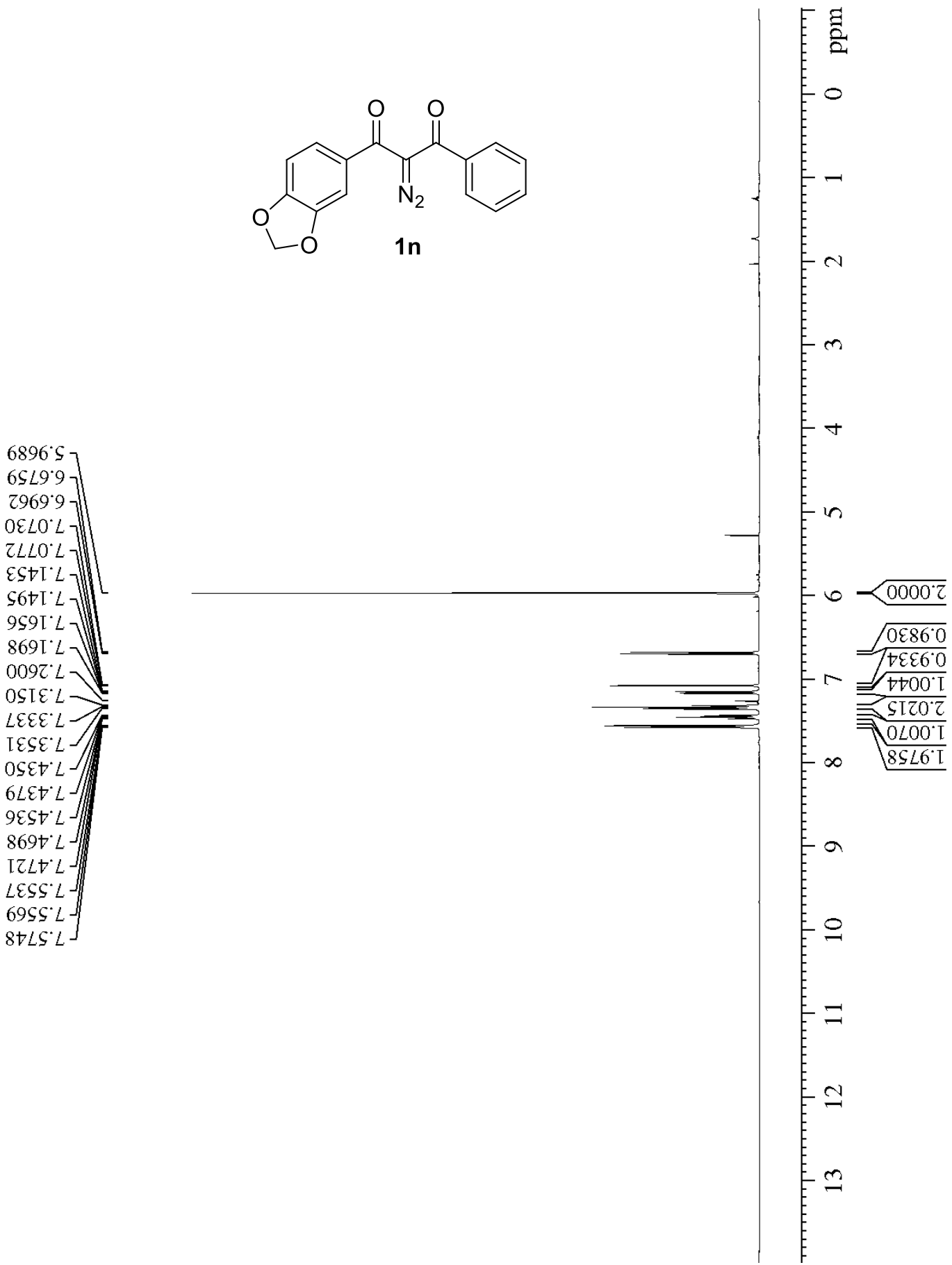

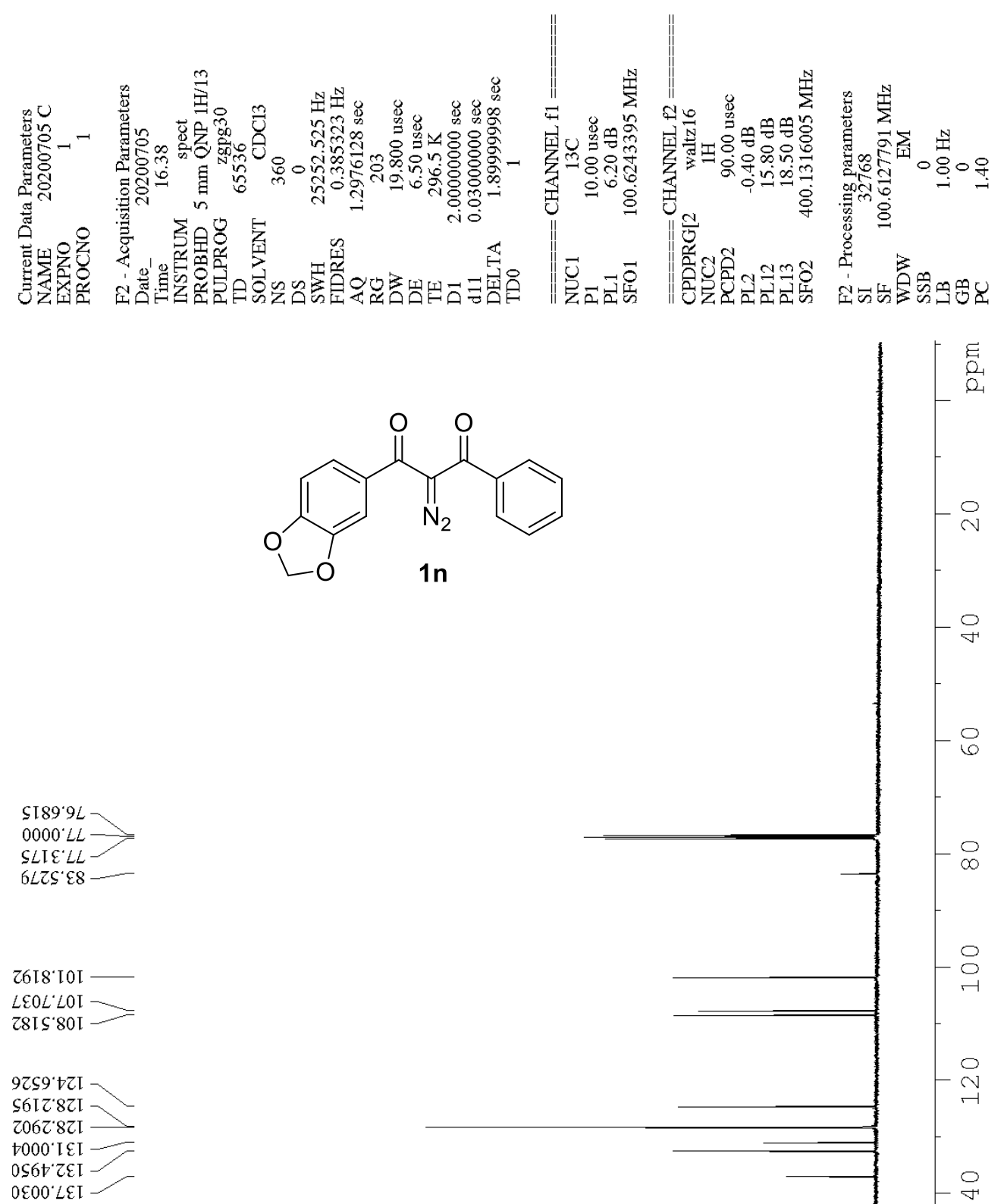<smiles>N=C(C(=O)c1ccccc1)C(=O)c1ccc2c(c1)OCO2</smiles>

$6 \varepsilon L L L D I-$

80St'ISI -

$\varepsilon 29 \varsigma^{\circ} \circ 8 \mathrm{I}-$ 6 6 $^{\circ} 98 \mathrm{I} \longrightarrow$

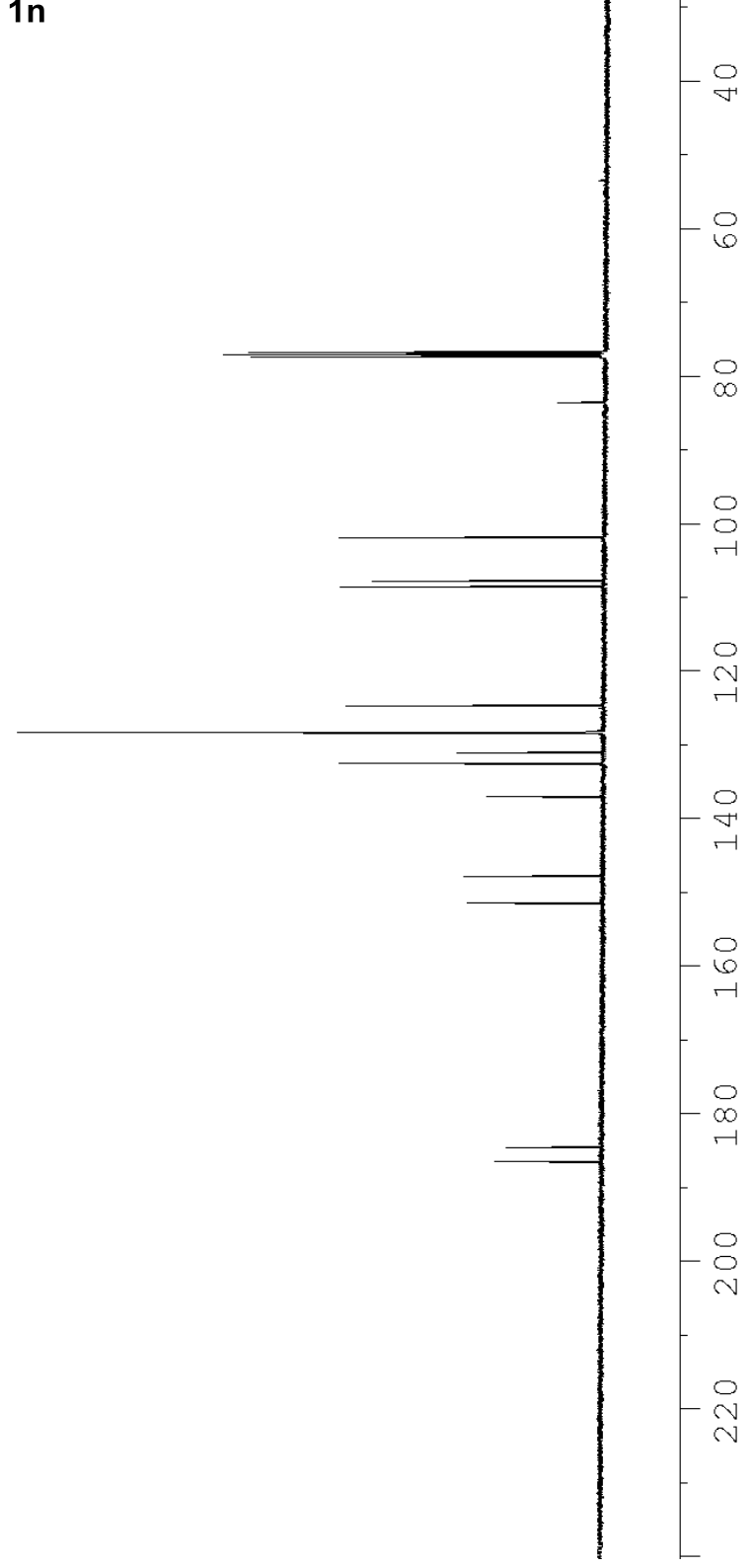




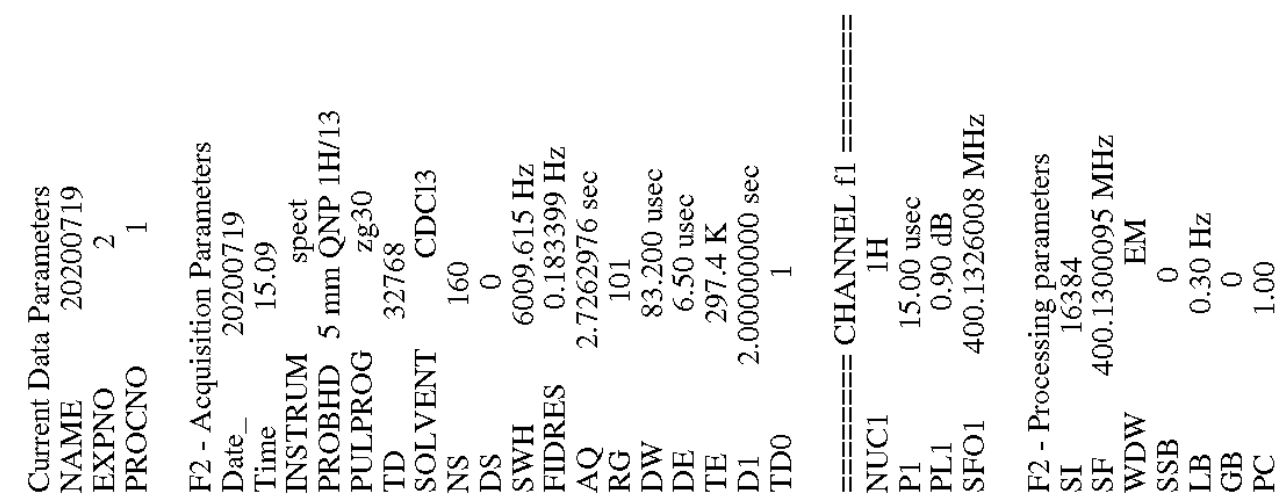<smiles>N=C(C(=O)c1ccccc1)C(=O)c1ccc2ccccc2c1</smiles>
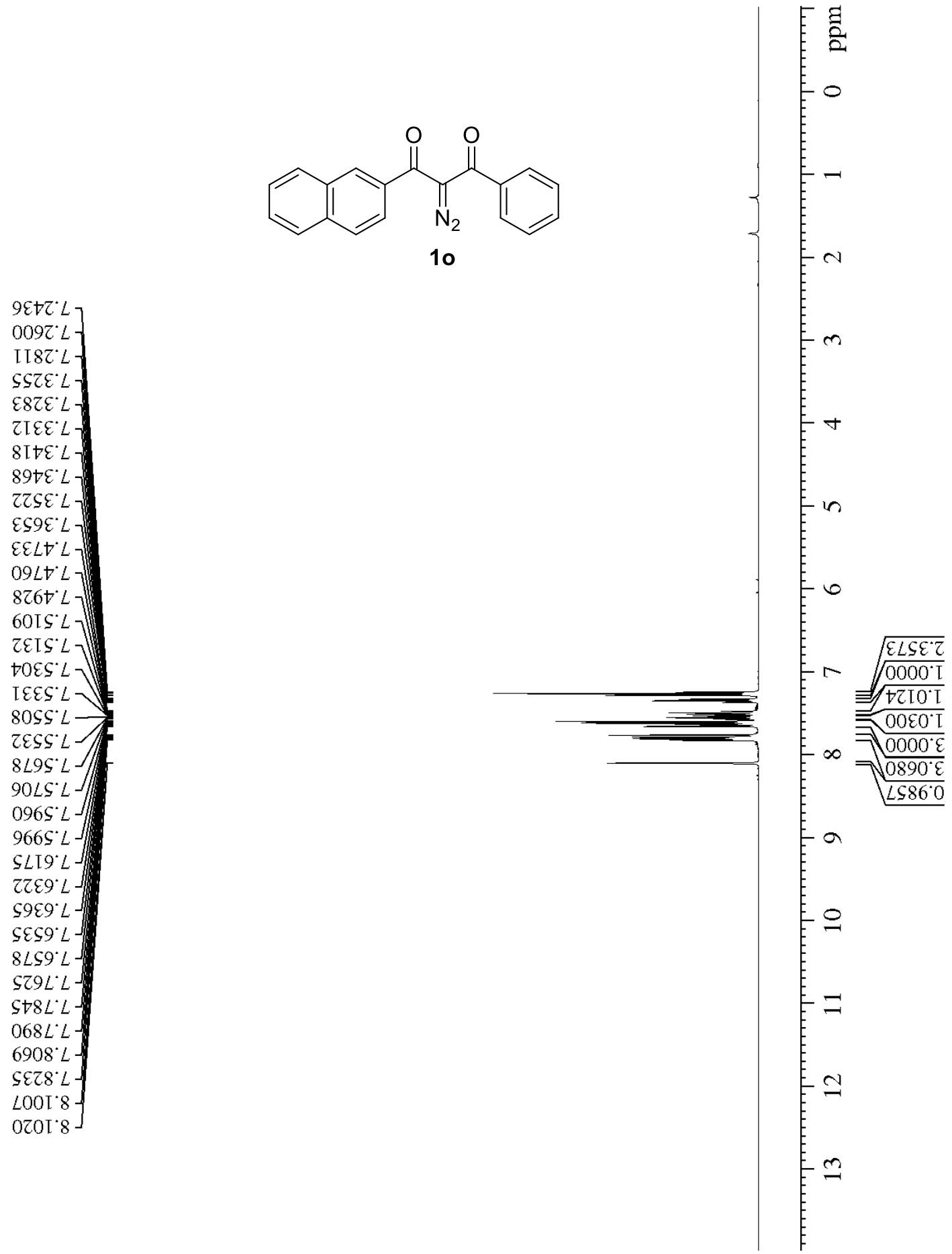


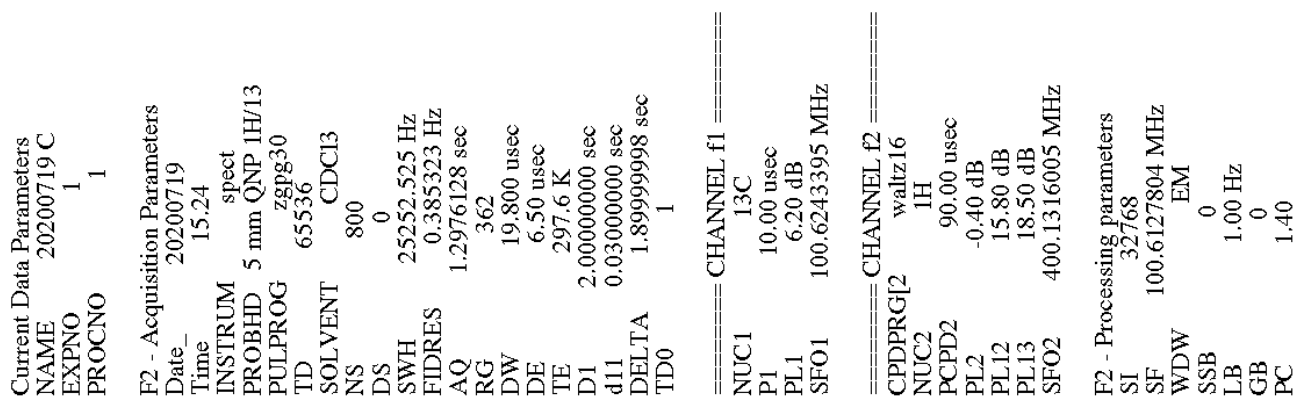

SI89.9L

$0000^{\circ} \mathrm{LL}$

TI0Z'LL

ELIE'LL

$\varepsilon 09 \varepsilon^{\circ} \vdash 8$<smiles>N=C(C(=O)c1ccccc1)C(=O)c1ccc2ccccc2c1</smiles>

10

$\angle 66 \mathrm{I}^{\circ} \mathrm{t} \mathrm{TI}$

I69L' $9 \mathrm{ZI}$

$86 z 28 \mathrm{ZZI}$

$\forall Z t \tau \cdot 8 Z I$

$690 \varepsilon^{\prime} 8 \mathrm{ZI}$

L680'6ZI

I $\varepsilon 08^{\circ} 6 \mathrm{ZI}-$

E066 IEI -

sSSt'ZEI

$\downarrow \downarrow \varepsilon\left[{ }^{\circ} \downarrow \varepsilon[-\right.$

${ }^{26 S 0}{ }^{\circ} \subseteq \mathcal{E} \mathrm{I}$

$9 S \angle 6^{\circ} 9 \mathcal{E L}$

เยIZ.98I

0Ztt'98I $\longrightarrow$

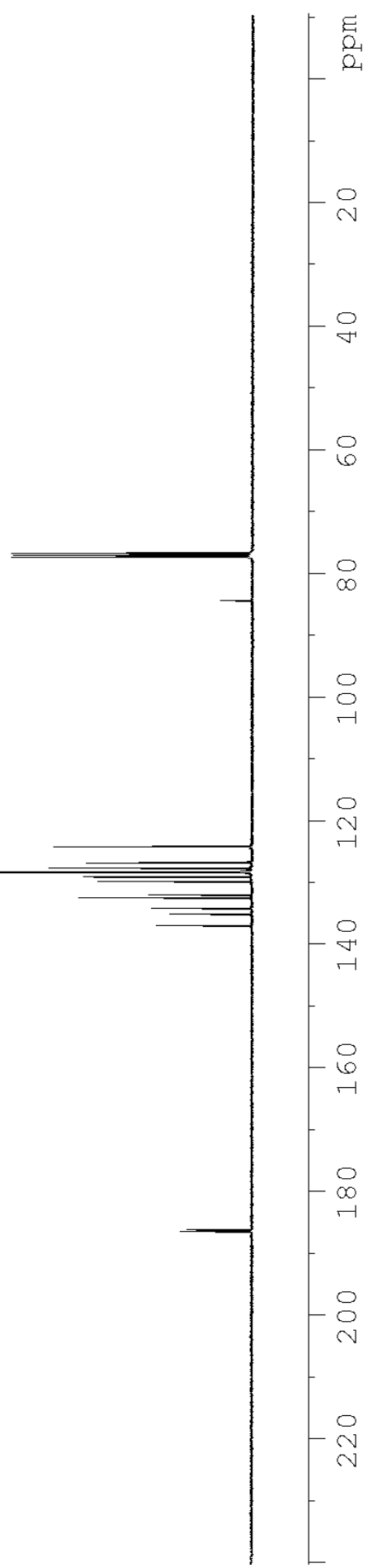



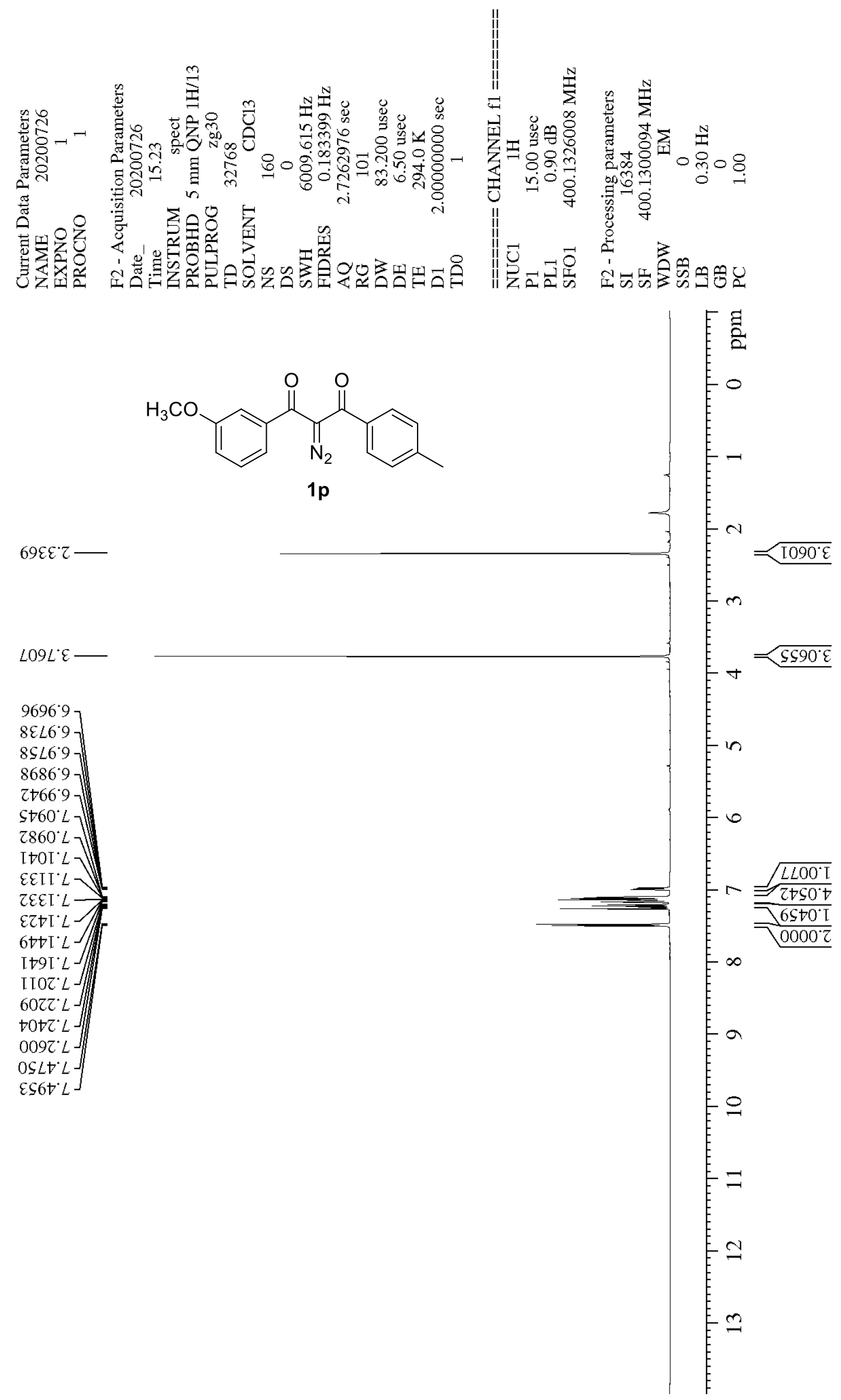


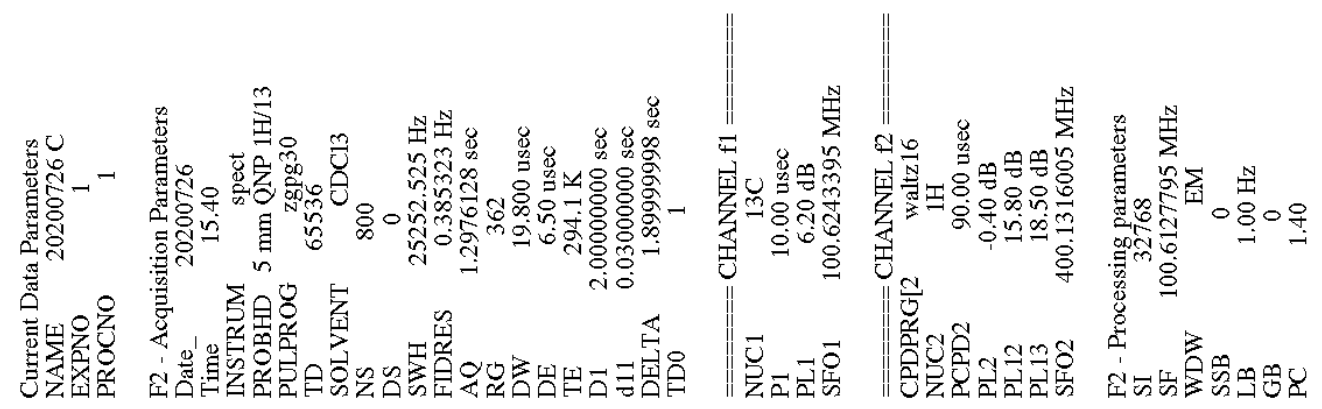

IOSS'IZ<smiles>COc1cccc(C(=O)C(=N)C(=O)c2ccc(C)cc2)c1</smiles>

LZIE'SS

$0789^{\circ} 9 L$

$0000^{\circ} \mathrm{LL}$

ZZOZ:LL

6LIE' $L L$

$9 \mathcal{E} 8 L \mathcal{E} \&$

S0Z8'ZI I

2026.8I

$26 \mathrm{I} L^{\circ} 0 \mathrm{ZI} \longrightarrow$

S89.'8ZI

tS06.8ZI -

$066 \mathrm{Z}: 6 \mathrm{ZI}$

$z 88 \mathrm{I} \cdot t \varepsilon \mathrm{I}$

Z89I' $8 \mathcal{E}$ I

$6687^{\circ} \mathcal{E} \mathrm{I} \mathrm{I}$

$8 S_{0} t^{\circ} 6 S I$

tI60.98I $\longrightarrow$

06SI'98I

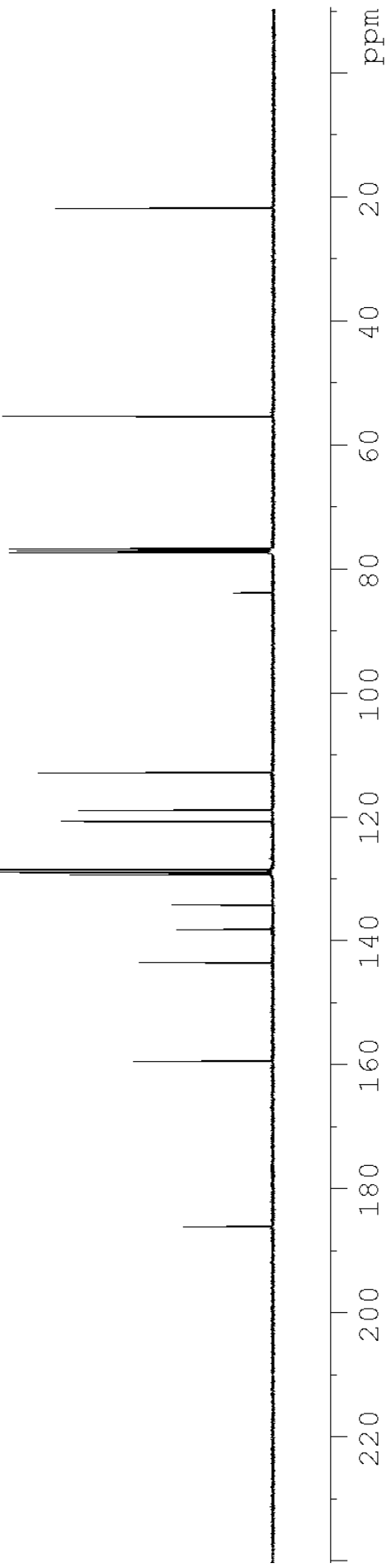




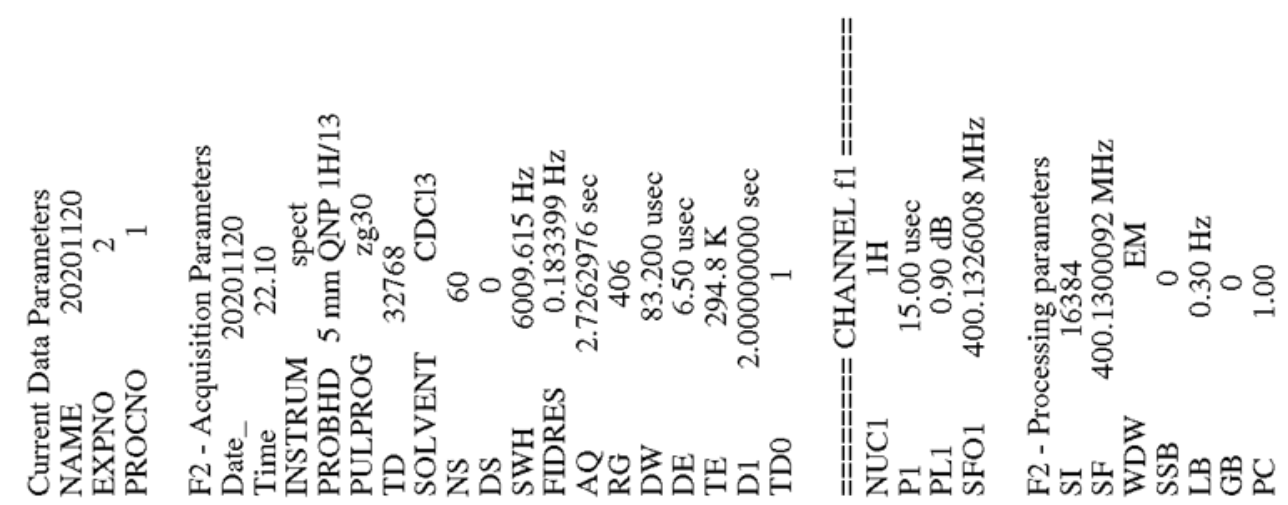

$88+\varepsilon \tau$<smiles>Cc1ccc(C(=O)C(=N)C(=O)c2cccc([N+](=O)[O-])c2)cc1</smiles>

$1 q$

$0 \varepsilon t I^{\circ} L$

629 ' $L$

$0092: L$

$88 \angle T^{\circ} L$

$2660^{\circ} L$

OtOS $L$

$2 t \tau S^{\circ} L$

$[t t S[]$

$\left.\angle 8 S 8^{\circ} \mathrm{L}\right]$

$\left.\checkmark S \angle 8^{\circ} \mathrm{L}\right]$

$08 \angle 8^{\circ} L$

I $188^{\circ} \mathrm{L}$

2292:8

SL9Z: 8

$6692: 8-$

$628 \tau: 8$

IS87:8-

2882:8-

2062:8

$00 t \varepsilon: 8$

SttE: 8

26tย:8

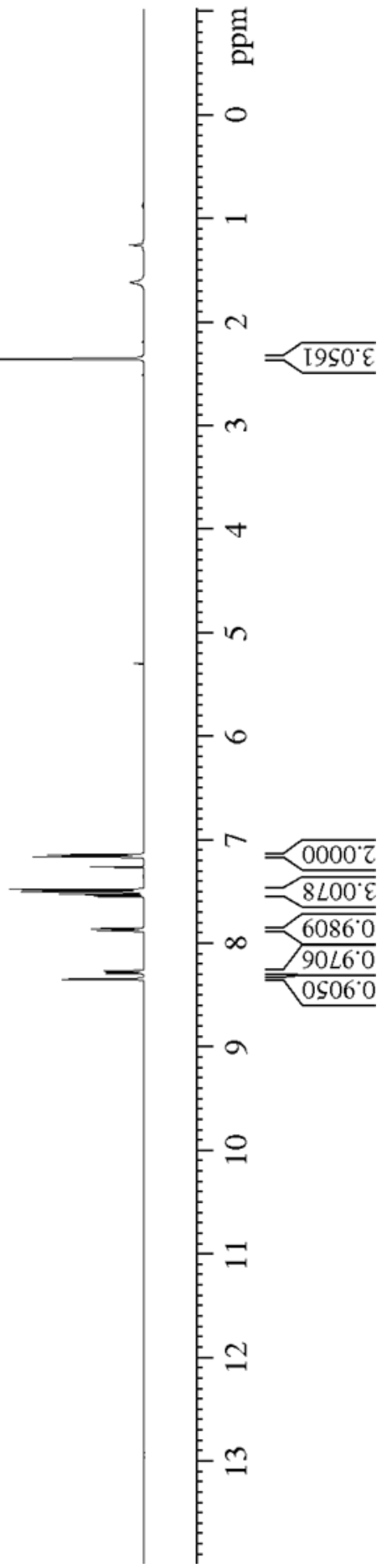




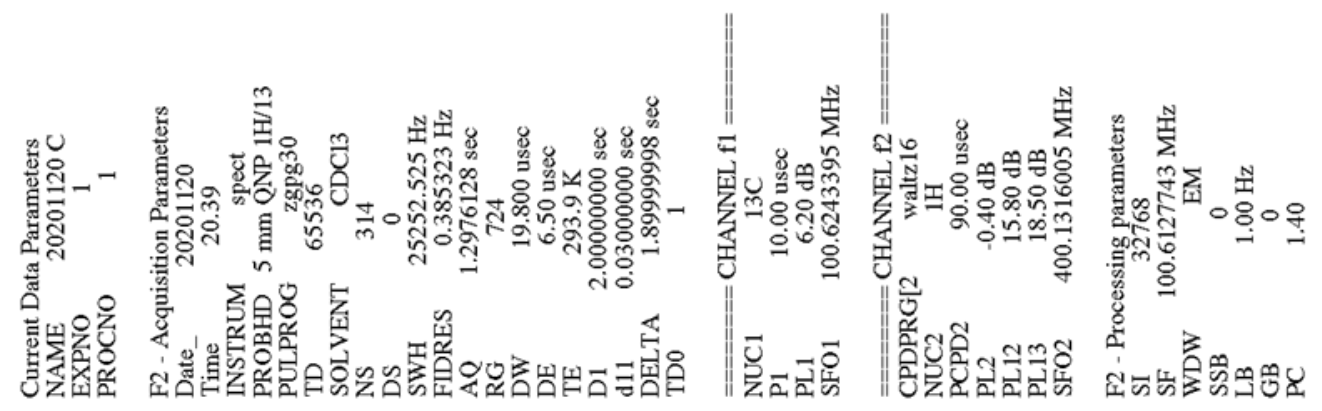

L009'IZ<smiles>Cc1ccc(C(=O)C(=N)C(=O)c2cccc([N+](=O)[O-])c2)cc1</smiles>

$8189^{\circ} 9 L$

I000 $L L$

$0 L I E ' L L$

$566^{\circ} t 8$

L8ES'EZI

ILSt'9ZI

$06 \$ Z$ '8ZI

$\angle \forall \angle Z^{\circ} 6 Z \mathrm{I}$

L $9 \varepsilon^{\circ} 6 Z \mathrm{I} \longrightarrow$

IZZL'EE I

EOS6 $\mathcal{E} \mathcal{~ I ~}$

$9 L \mathrm{tt} 8 \varepsilon \mathrm{I}$

$8 \mathrm{I}^{\circ} \mathrm{I}, \mathrm{VI}$

6I $29^{\circ} \mathrm{LDI}$

$68 \mathrm{I} L ' t 8 \mathrm{I}$

I980. $\mathrm{SI}>$

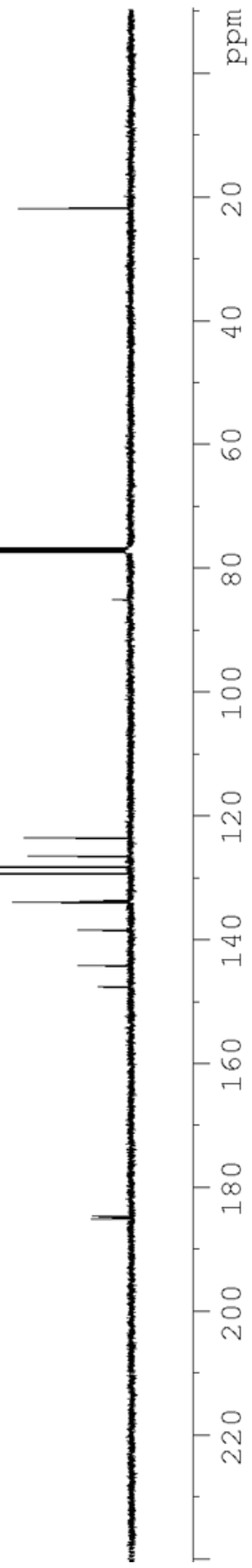



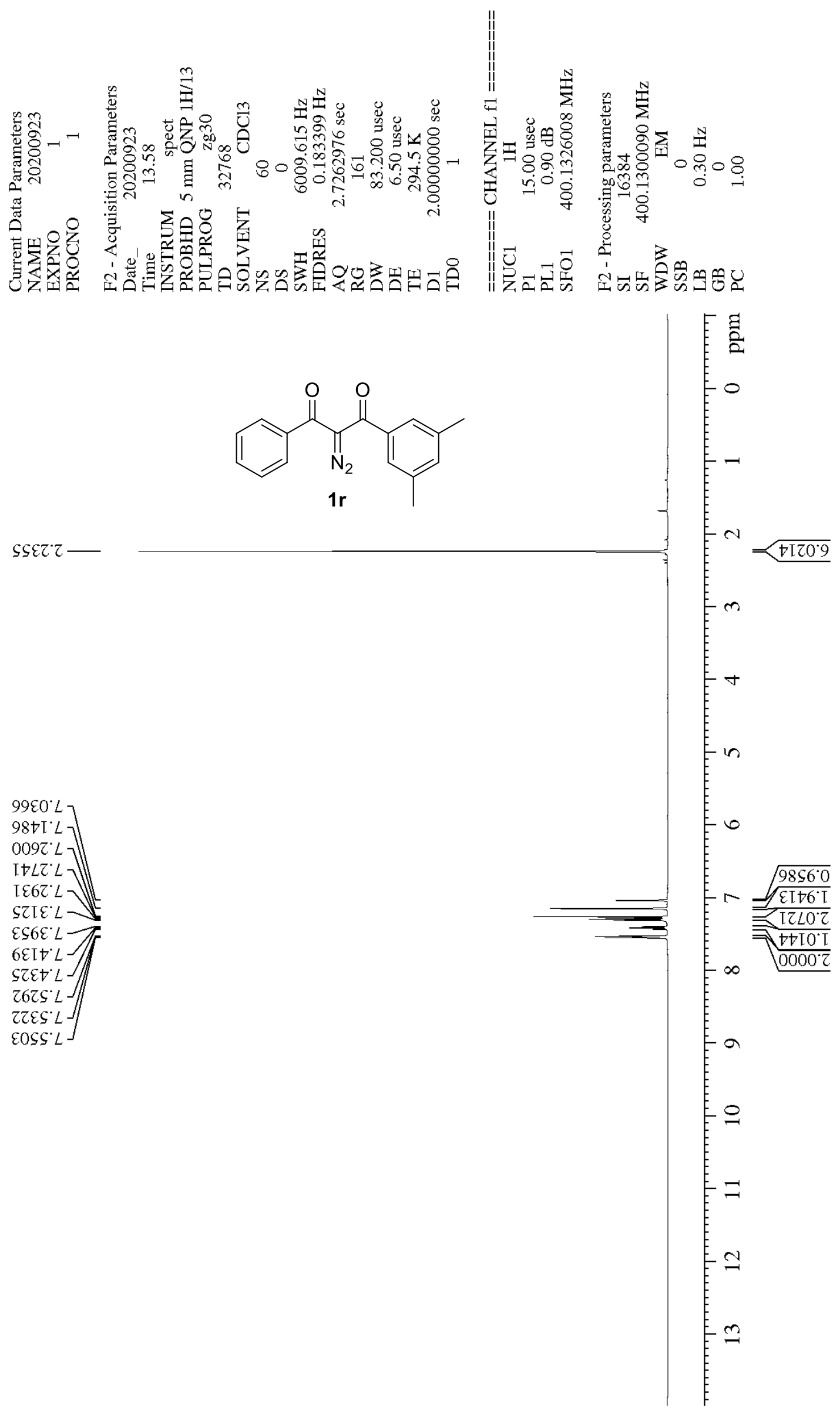


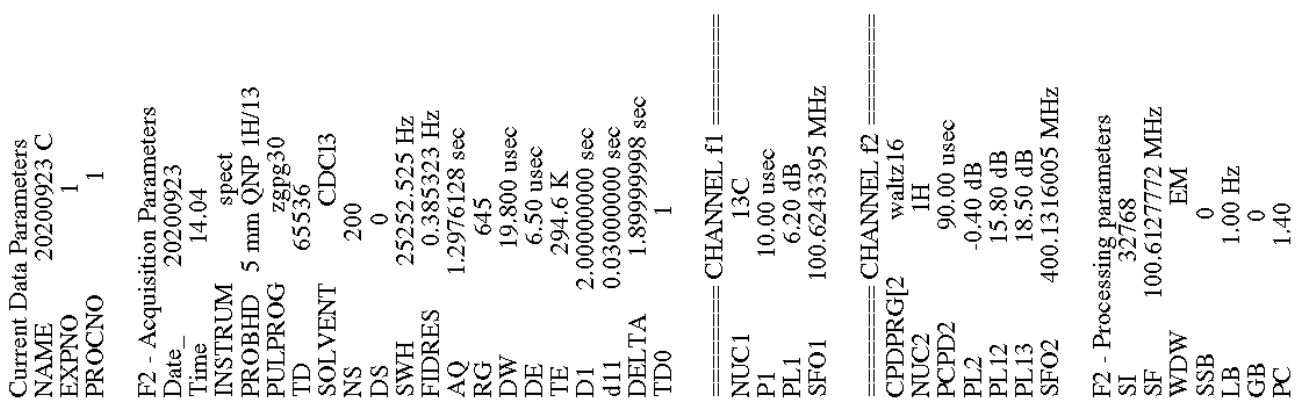

$\varepsilon 6 L 6^{\circ} 02$<smiles>Cc1cc(C)cc(C(=O)C(=[W])C(=O)c2ccccc2)c1</smiles>

$2789^{\circ} 9 L$

Z9LE' $Z E I$

$\left\llcorner\vdash 8 \mathrm{I}^{\circ} \in \textrm{I} \longrightarrow\right.$

$086 L^{\circ} 9 \varepsilon \mathrm{I}-$

69II'LEI -

LLOO'BEI

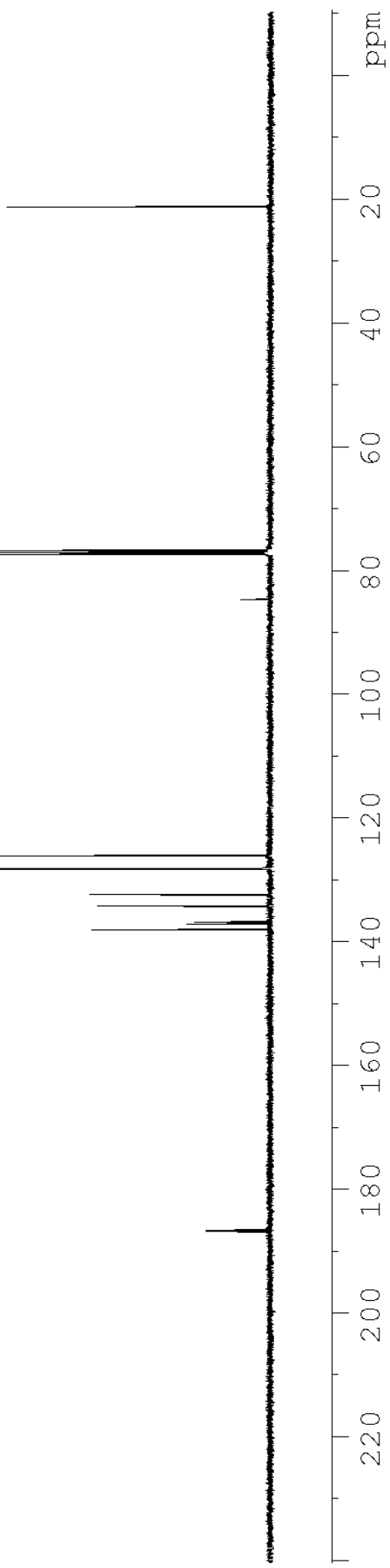




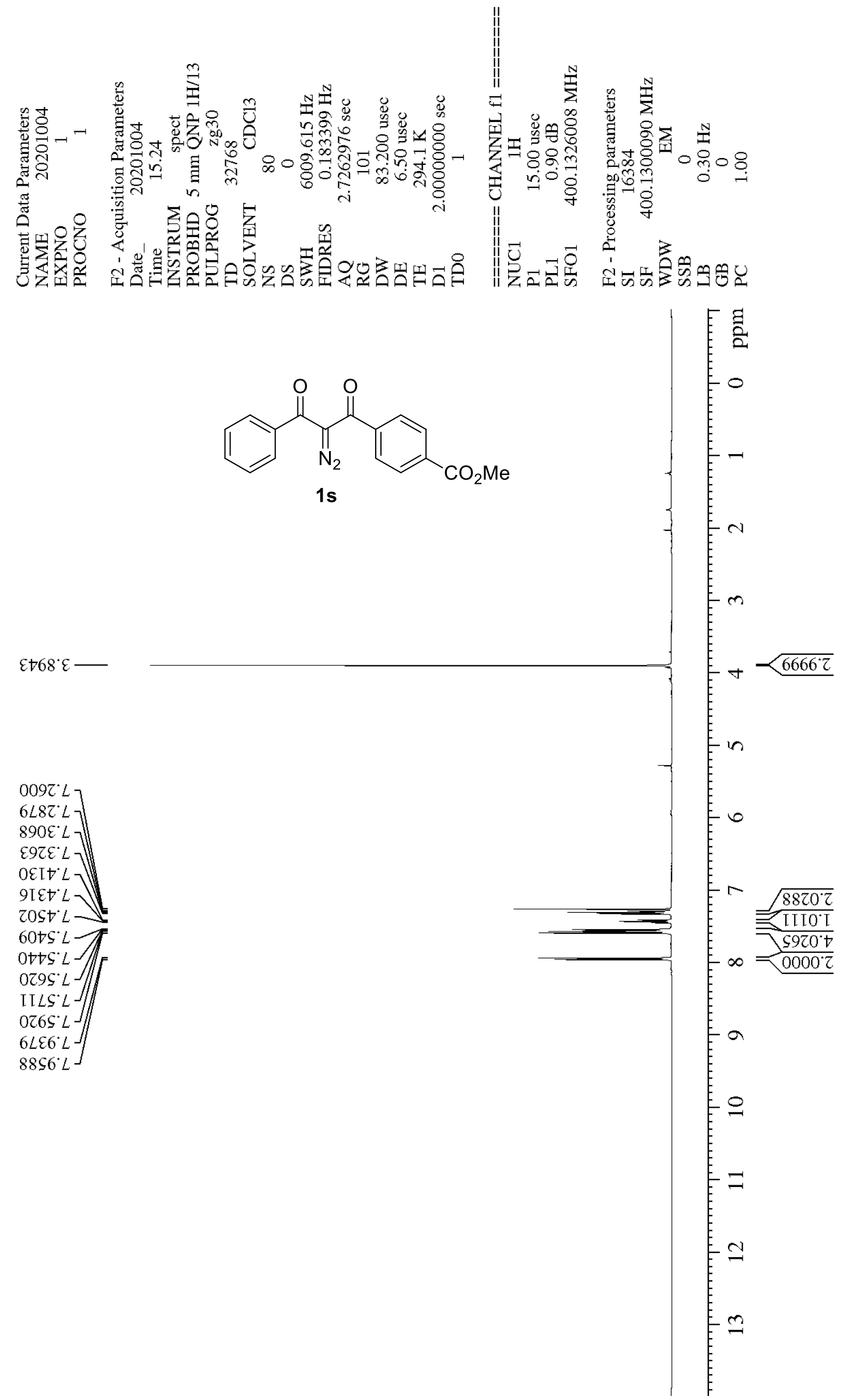




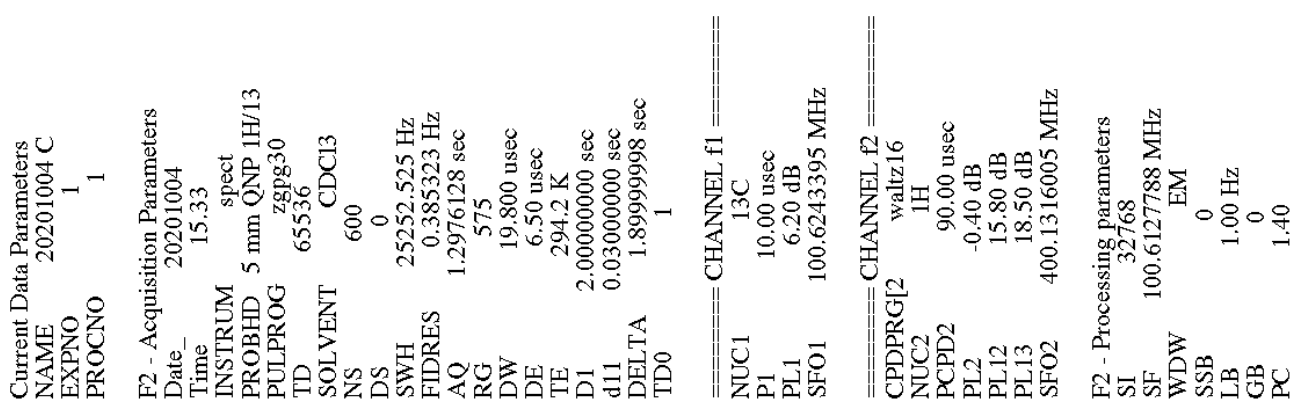

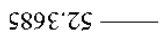

9โ89.9L

$0000 \mathrm{LL} \longrightarrow$

$9 L I E L L-$

$\varepsilon \angle t 6^{\circ}+8$<smiles>CCOC(=O)c1ccc(C(=O)C(=N)C(=O)c2ccccc2)cc1</smiles>

$1 \mathrm{~s}$

6SIZ'8ZI

Z๐Zt'8ZI -

$60+\varepsilon \cdot 62 \mathrm{I} \perp$

9Sz8. $z$

ZSZI' $\mathcal{E} I$

$8 † Z 9^{\circ} 9 \varepsilon 1$

OIS9.0tI

\668 $\ 9 \mathrm{I}$

$\angle t t 9 . \$ 8 \mathrm{I}$

$6+\angle 0^{\circ} 98 \mathrm{I} \longrightarrow$

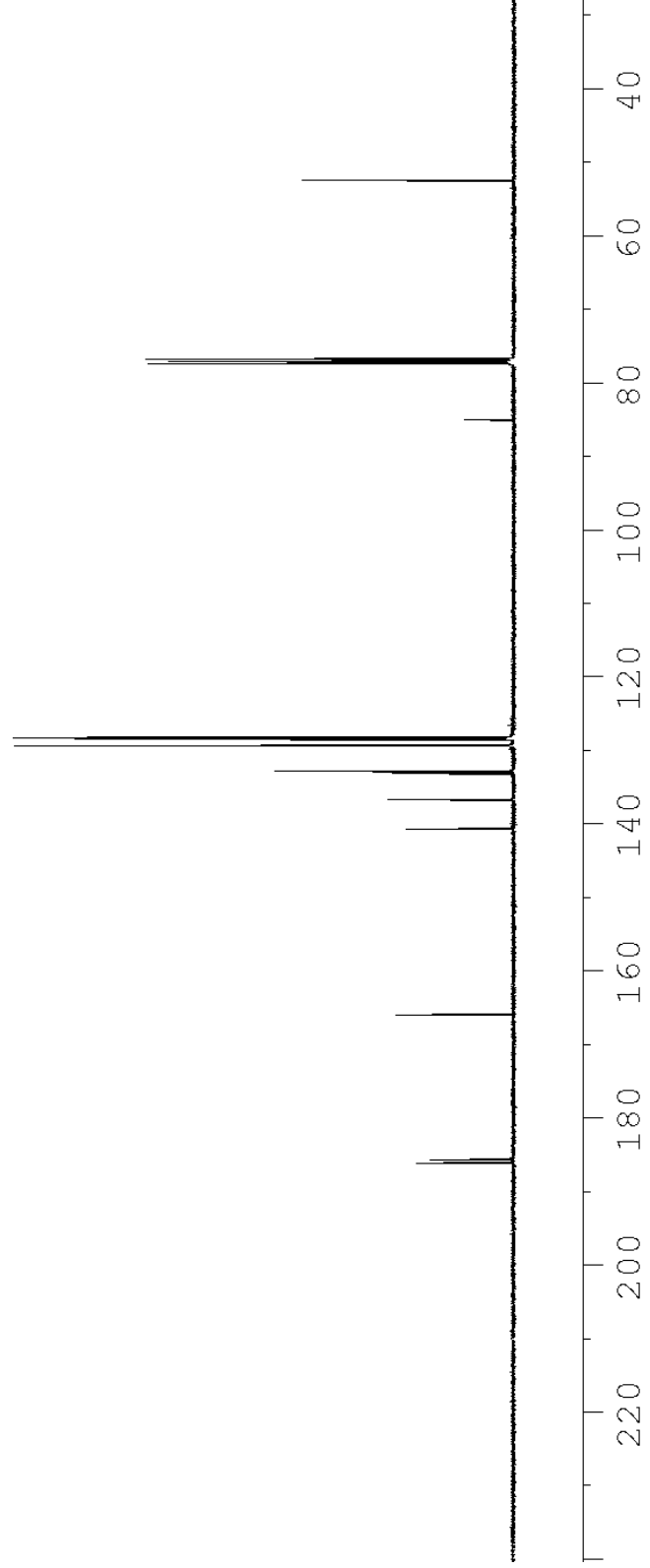



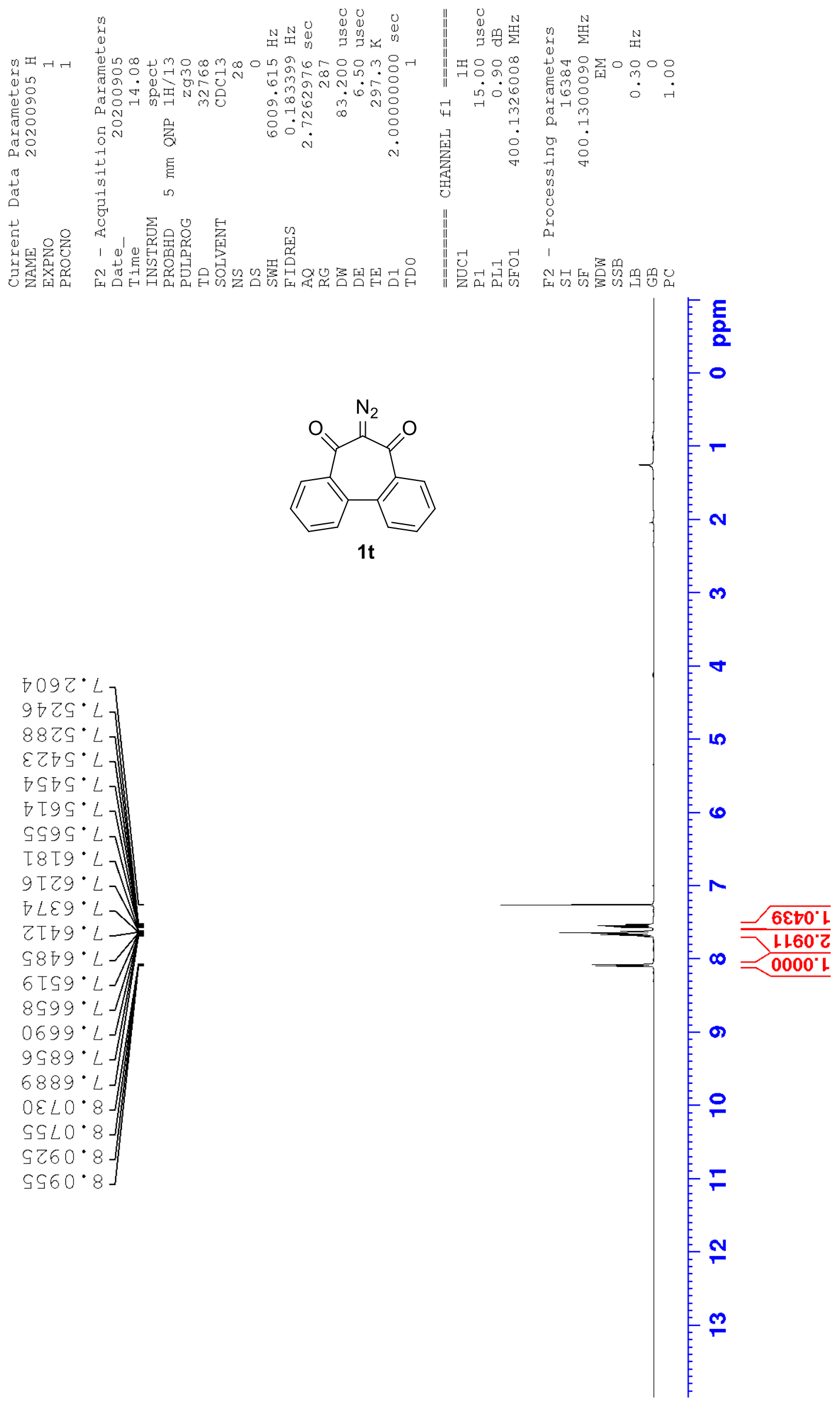

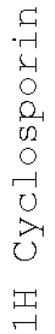



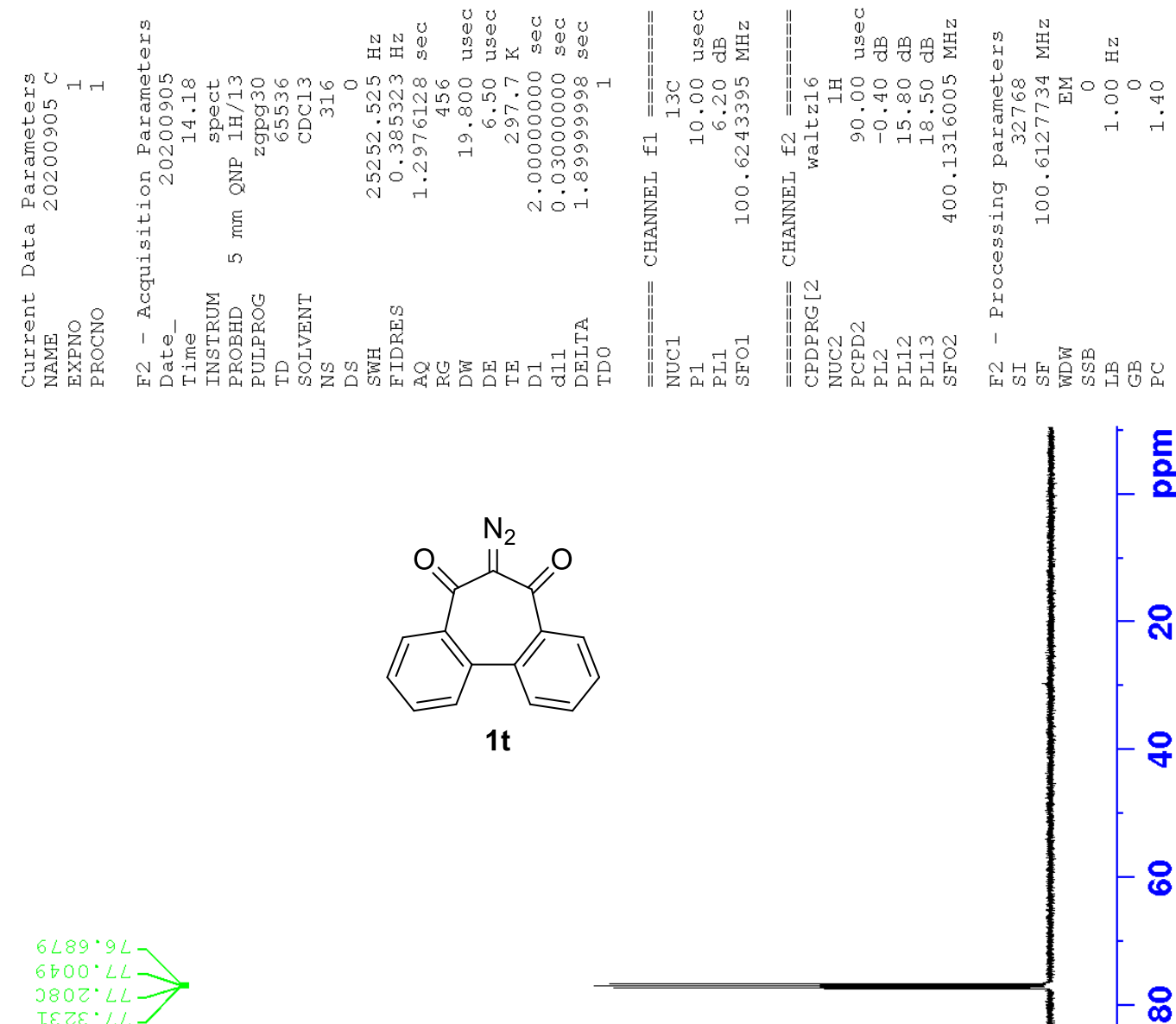

乙๐โ ${ }^{*} \varepsilon 6$
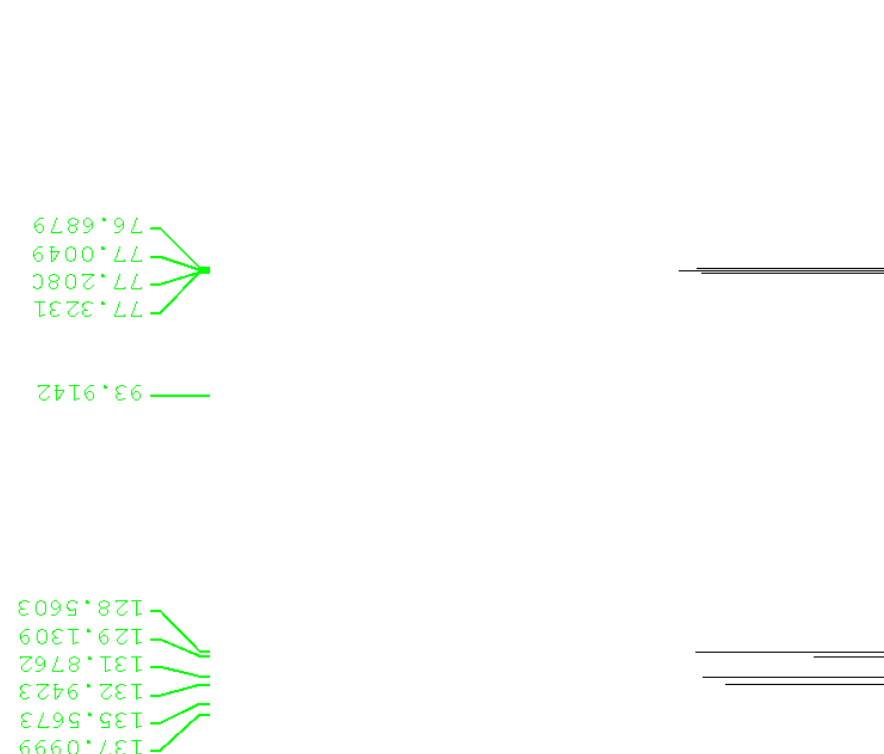

8

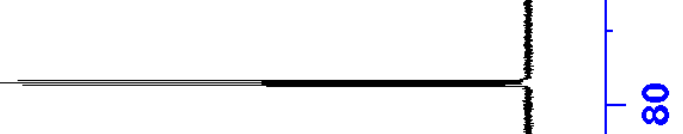

ZGSI* Z8I

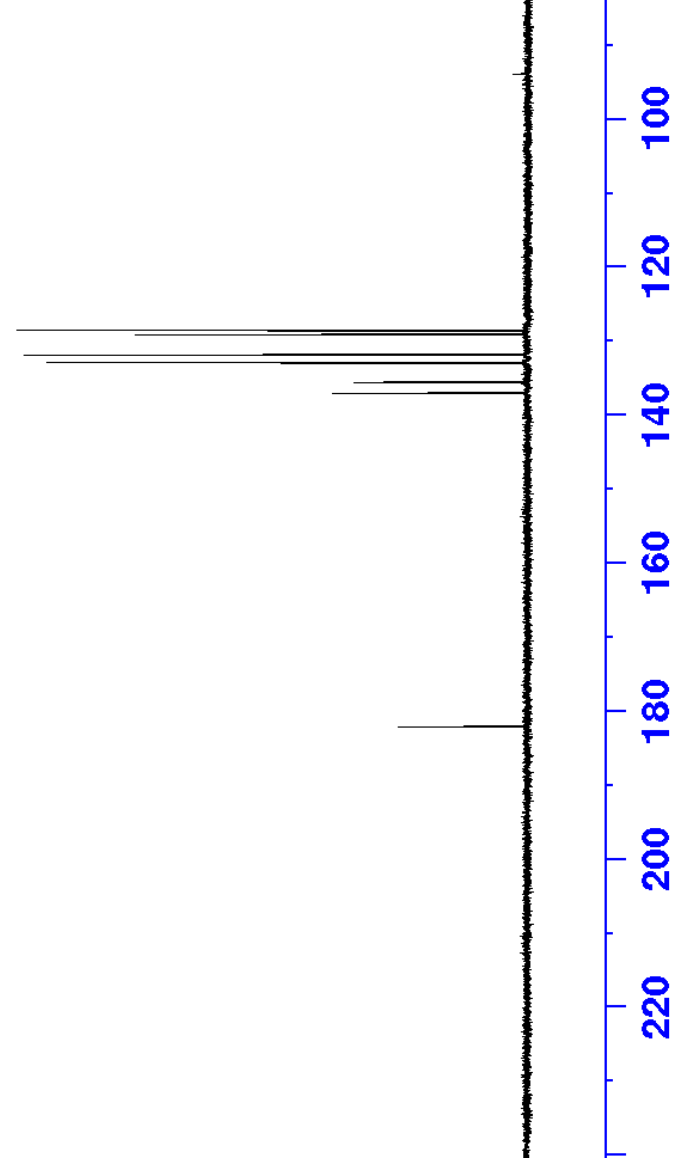



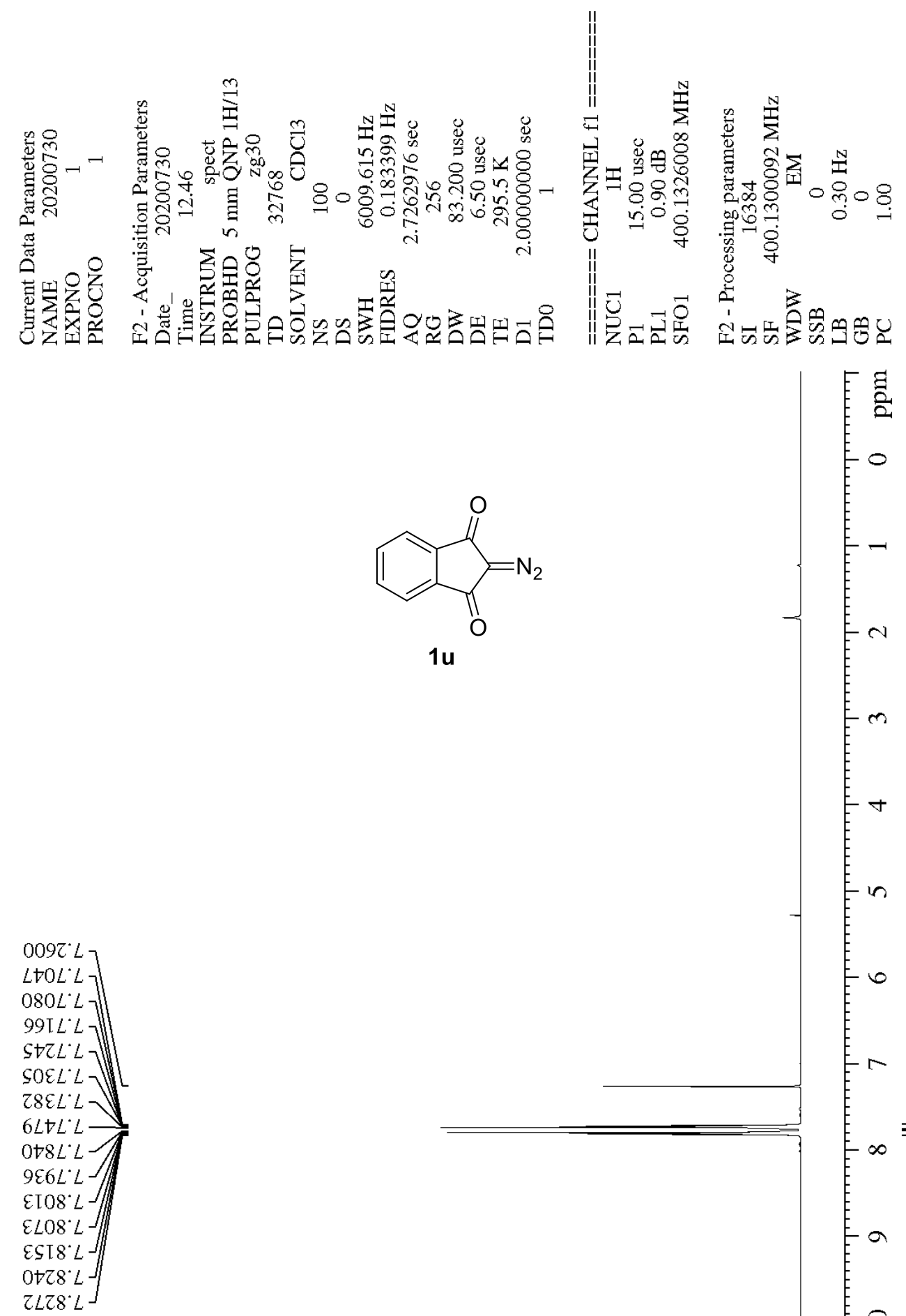<smiles></smiles>

1u

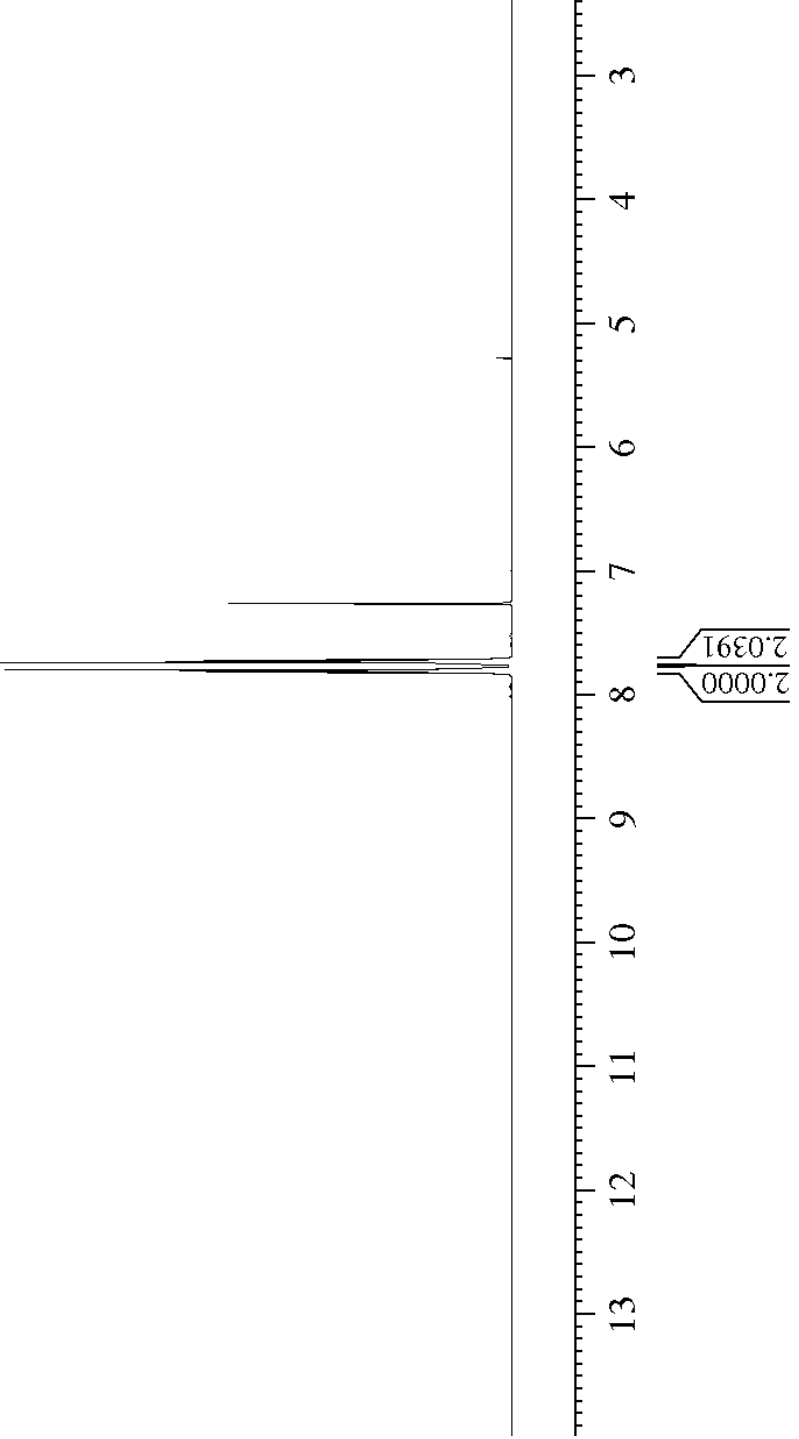



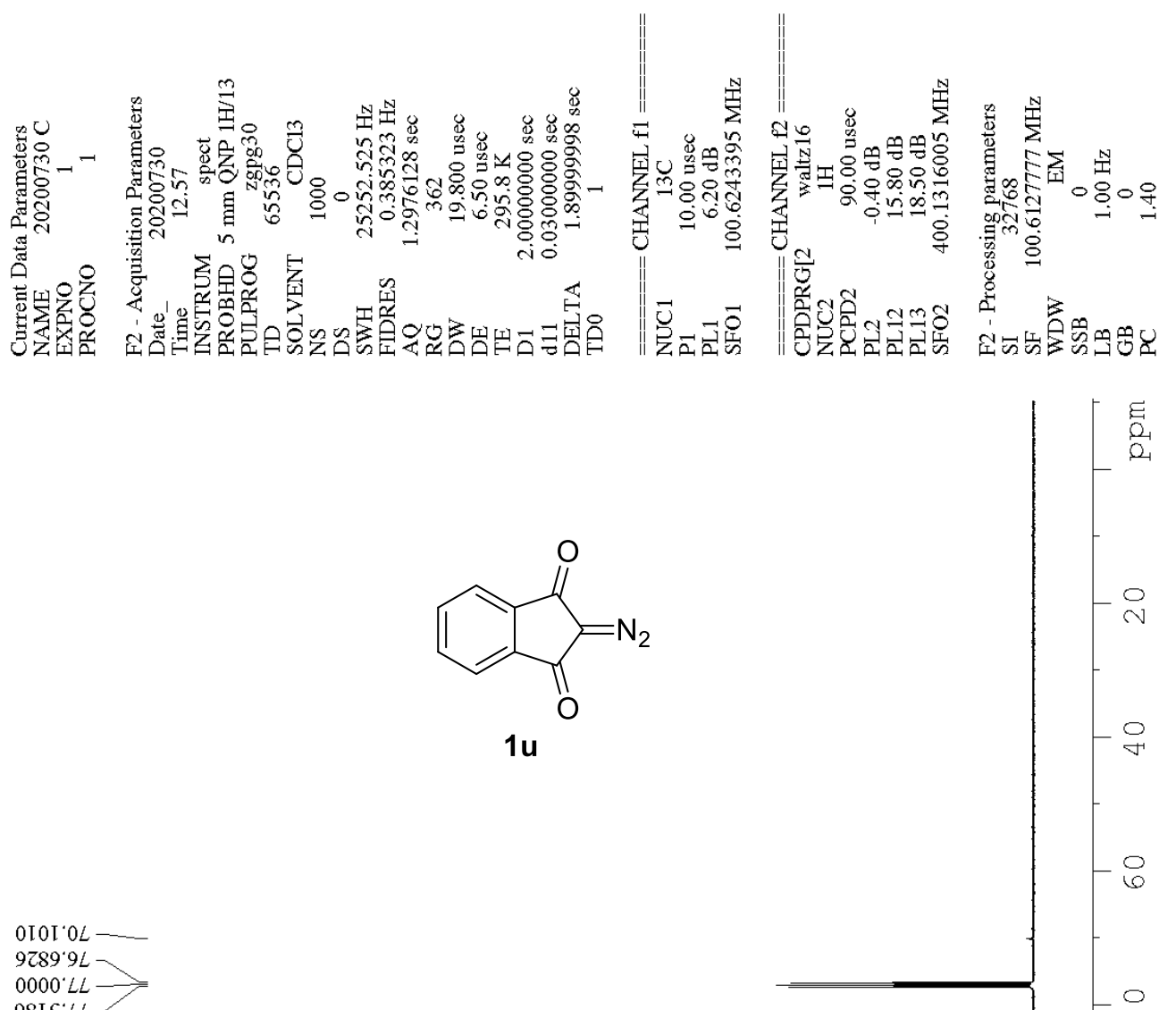

1u

\$929:Z2I -

ZSZL'tEI tOI $0^{\circ} L E I-$

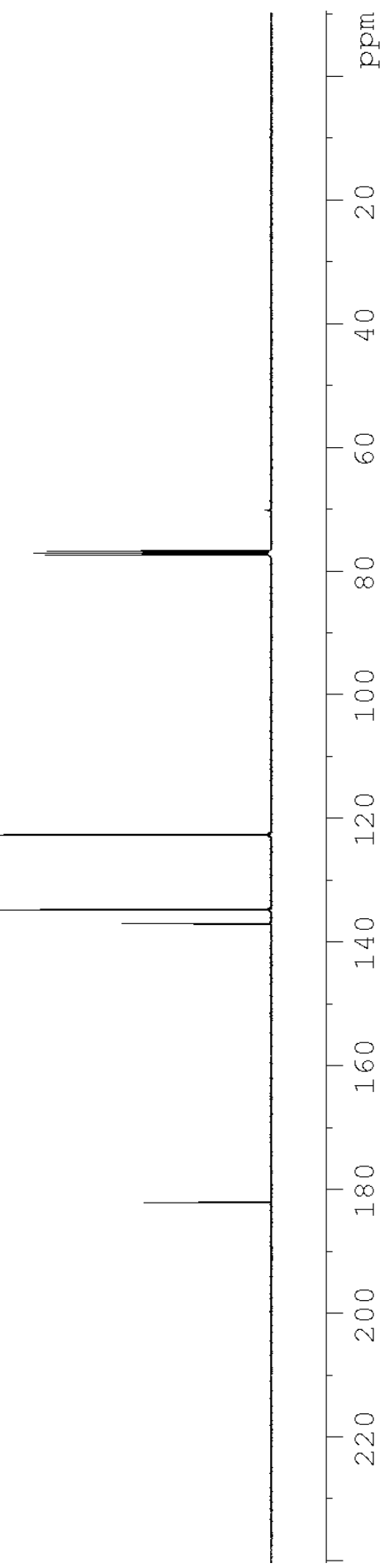



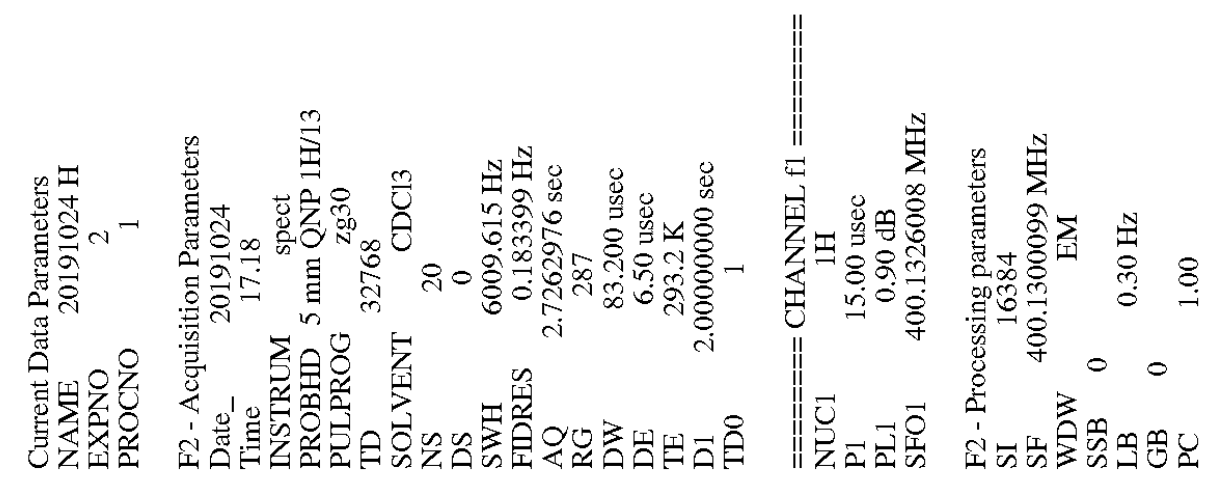<smiles>O=C(C(=O)c1ccccc1-c1ccccc1)c1ccccc1</smiles>

3a
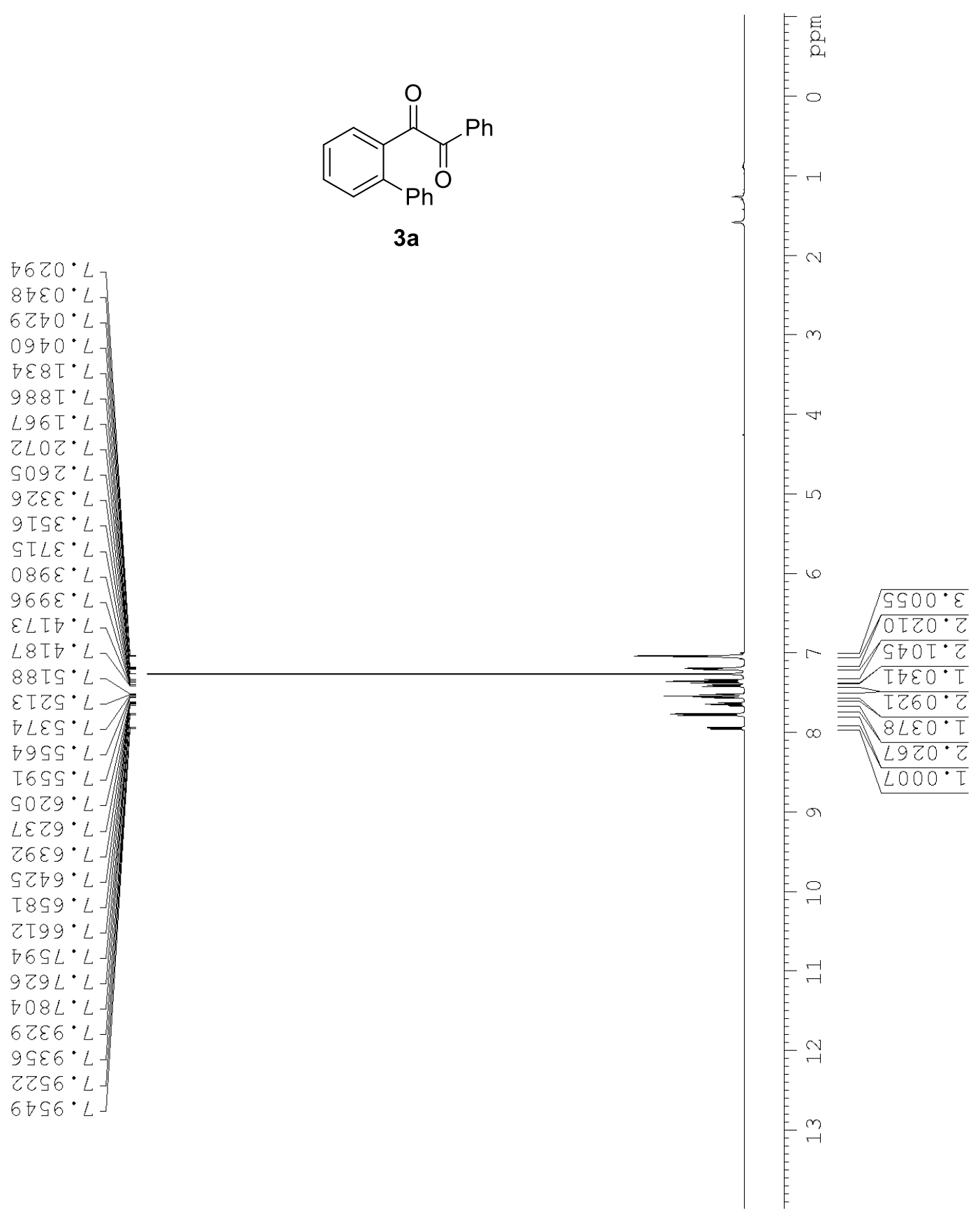


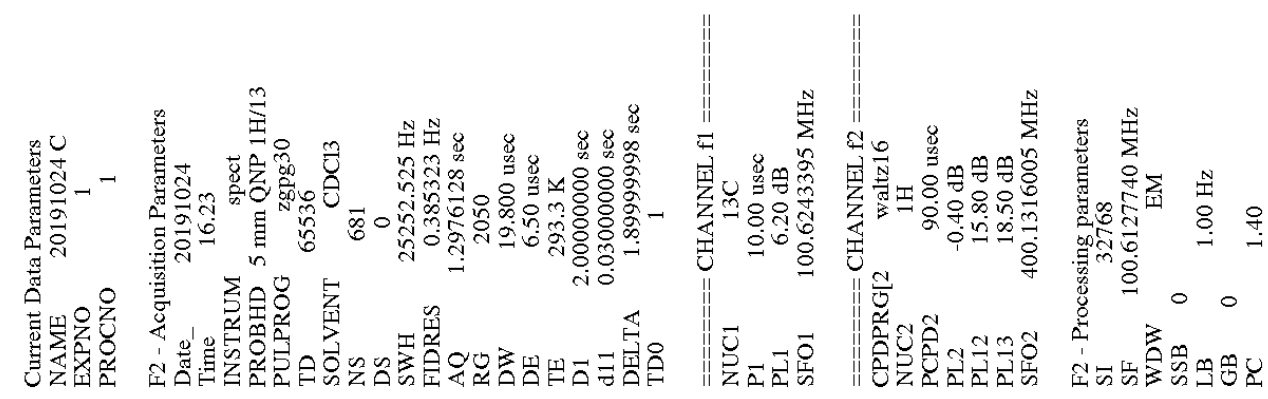<smiles>O=C(C(=O)c1ccccc1-c1ccccc1)c1ccccc1</smiles>

$3 a$

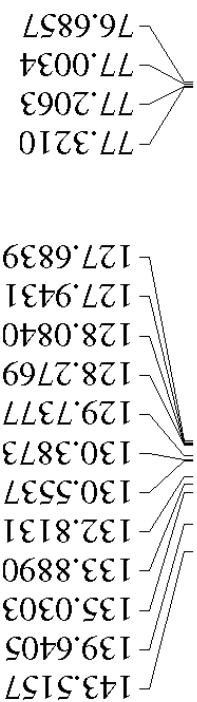

$\downarrow \$ 82^{\circ} \mathrm{I6I}-$

$\varepsilon+8 \varsigma^{\circ} \subseteq 6 \mathrm{I}-$

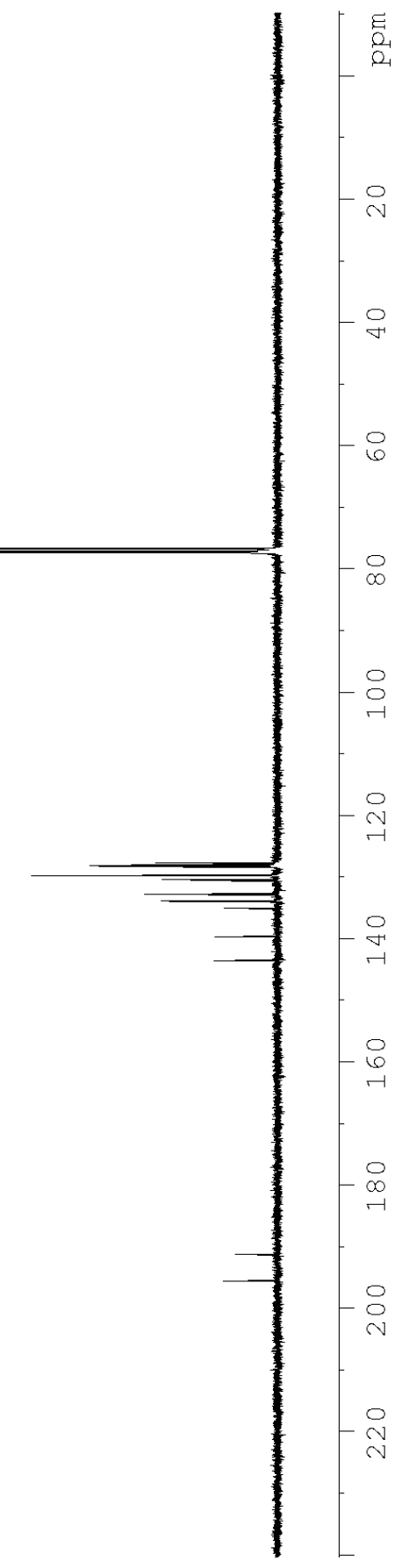




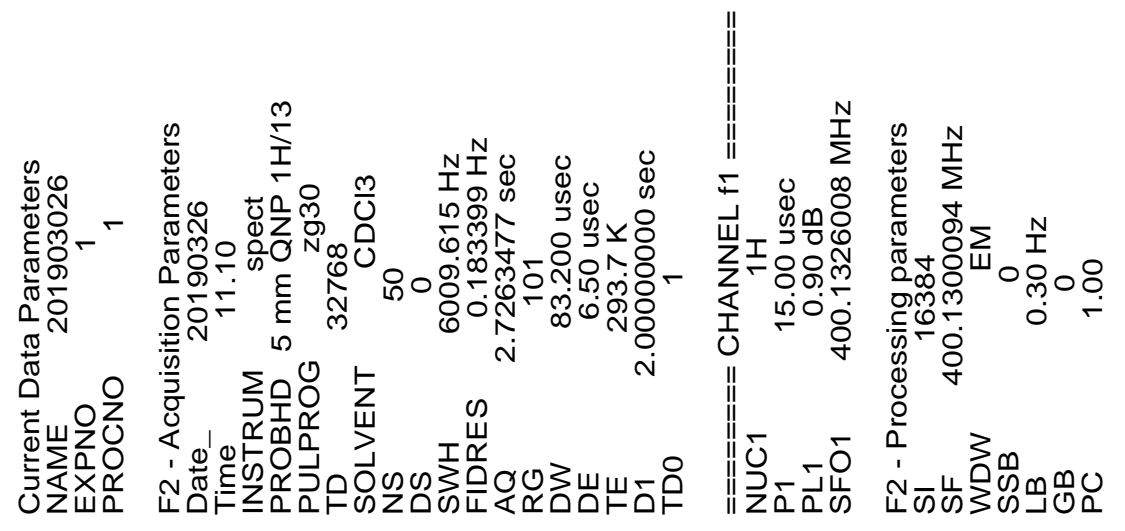<smiles>O=C(C(=O)c1ccccc1)c1ccccc1</smiles>

1092' $\angle$

$8060^{\circ}$

$8 E I S^{\circ} L$

टEES $L$

9ट†9 ${ }^{\circ} \mathrm{L}$

$6099^{\circ} L$

$86 \angle 9^{\circ} \mathrm{L}-$

$89966^{\circ} \mathrm{L}$

$8986^{\circ} \mathrm{L}$

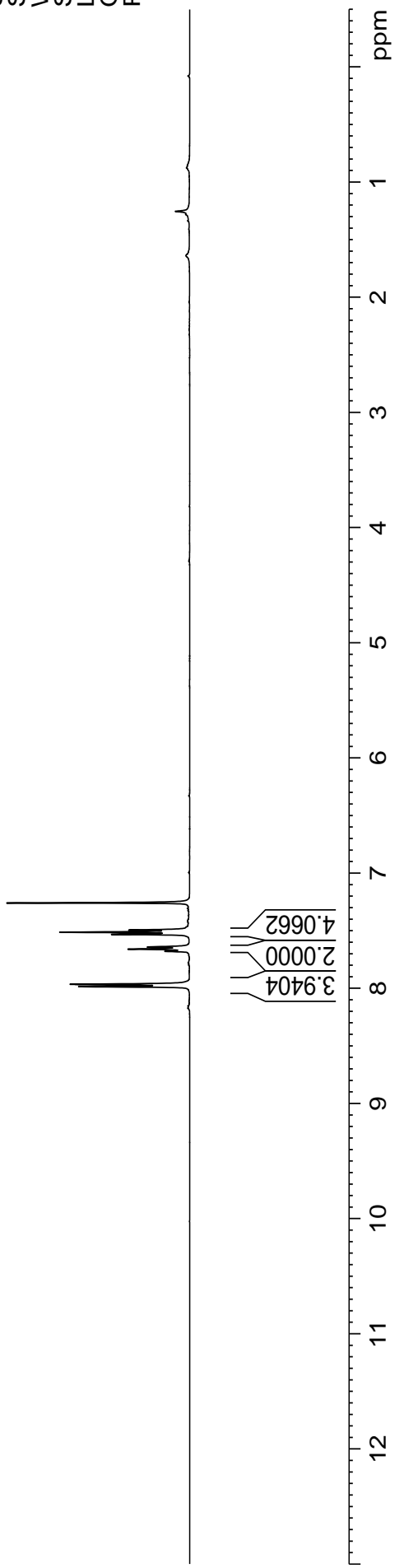



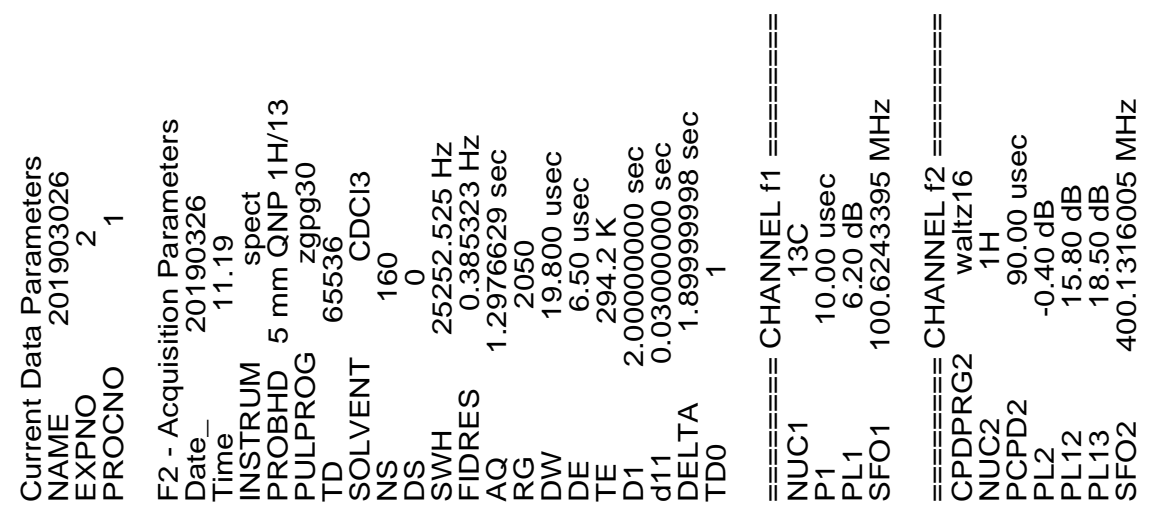

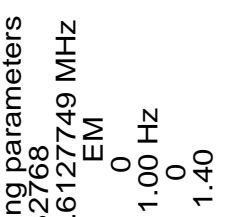

ज्रलें

응

3

300<smiles>O=C(C(=O)c1ccccc1)c1ccccc1</smiles>

$3 b$

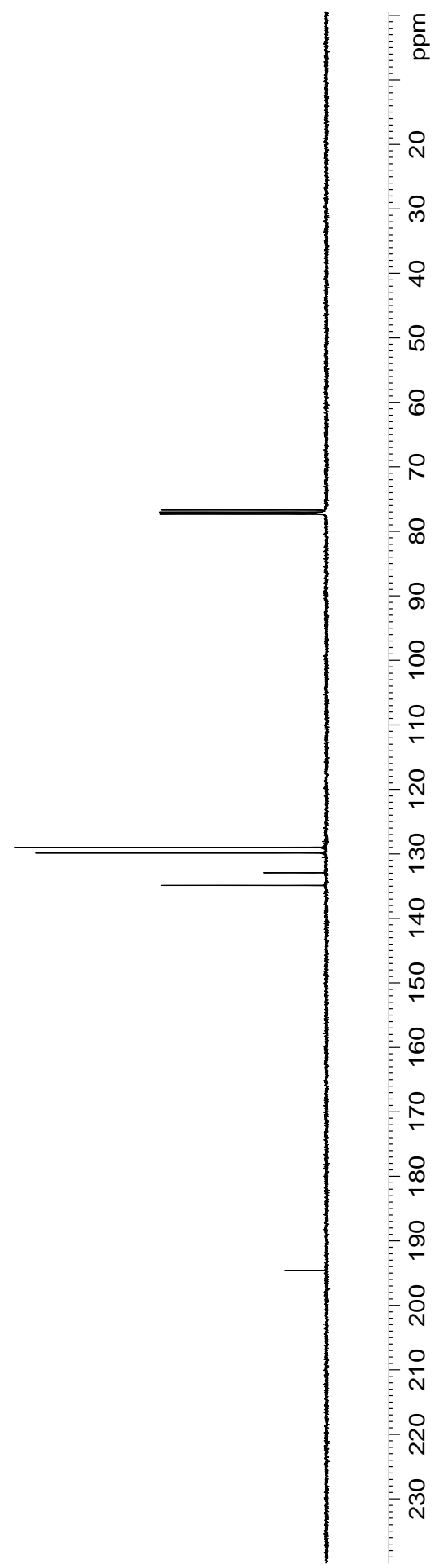

889.92

ट80ट L $>$

ธEZE: LL

9866.82

$8 \varepsilon \angle 8^{\circ} 62 \mathrm{~L}$

\&8เ6 ¿हเ

$0 \angle 88^{\circ} \nabla \varepsilon\llcorner$

6ง9ง'จ6เ- 


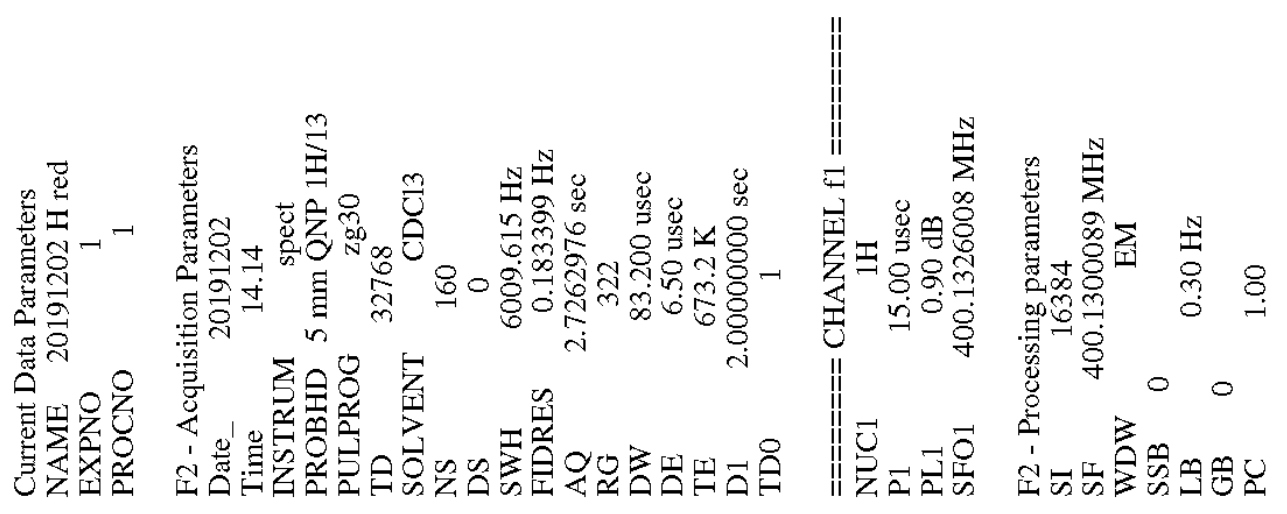<smiles>Cc1ccccc1C(=O)C(=O)c1ccccc1</smiles>

$\varepsilon I L L Z$

$9092^{\circ} L$

$\varepsilon 68 \tau^{\circ} L$

SOtE $L$

$S 6 S E L$

I LLF' $L$

I $6 L t^{\circ} L$

$686 t^{\circ} \mathrm{L}$

$\operatorname{IIZS}^{\circ} L$

$60 t S^{\circ} L$

EZE9. $L$

$9279^{\circ} \mathrm{L}$

OZS9 $9^{\circ} L$

I I $99^{\circ} \mathrm{L}$

$\angle 6 \angle 9^{\circ} \mathrm{L}$

$0 S 96^{\circ} \mathrm{L}$

I896 L

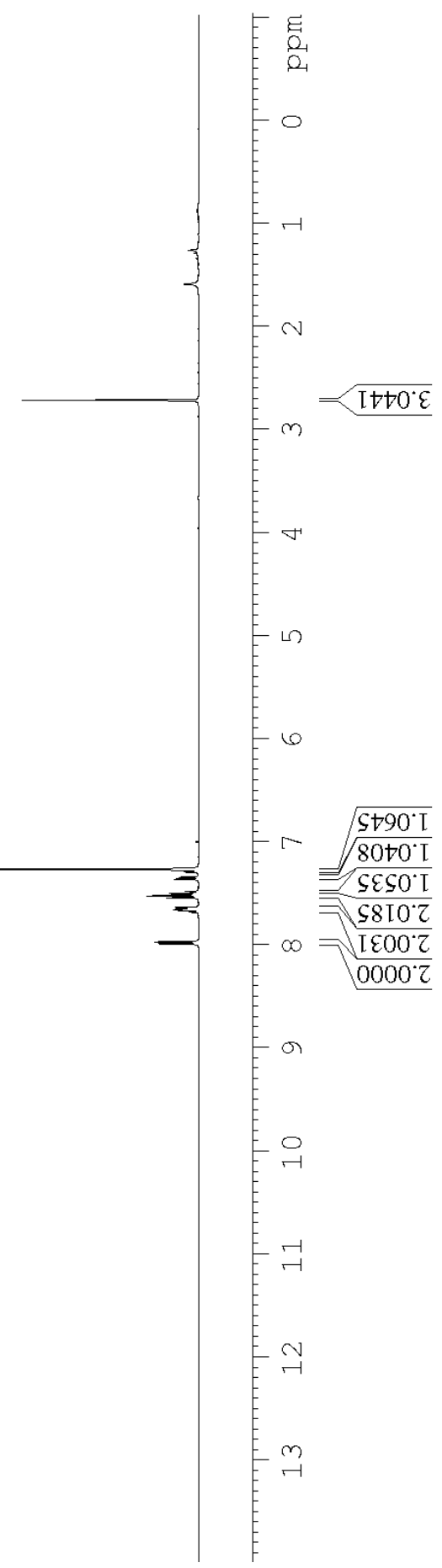




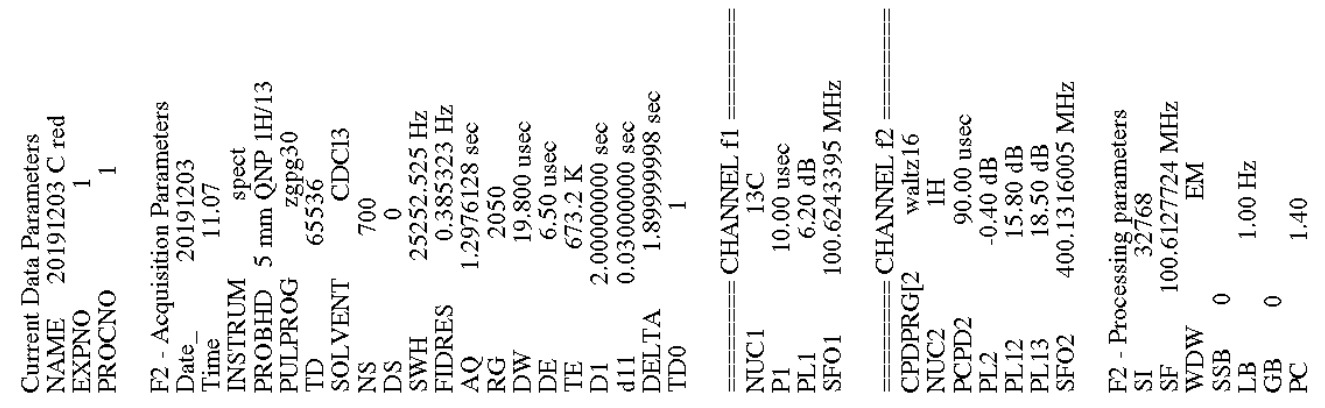

$\cos 6.12$<smiles>Cc1ccccc1C(=O)C(=O)c1ccccc1</smiles>

ZI69.9L

$9800^{\circ} \mathrm{LL}$

IZI $\angle L L$

S9ZE $L L$

L0Z0'9ZI

$\$ 600^{\circ} 6 \mathrm{ZI}$

6ZE6.6ZI

$0 L I L$ IEI

6L9S'ZEI

$6 \multimap 80^{\circ} \mathcal{E} \mathcal{I}$

EI08' $\mathcal{E}$ I

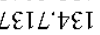

$890 t^{\prime}$ ItI -

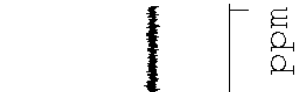

St8L'96I -

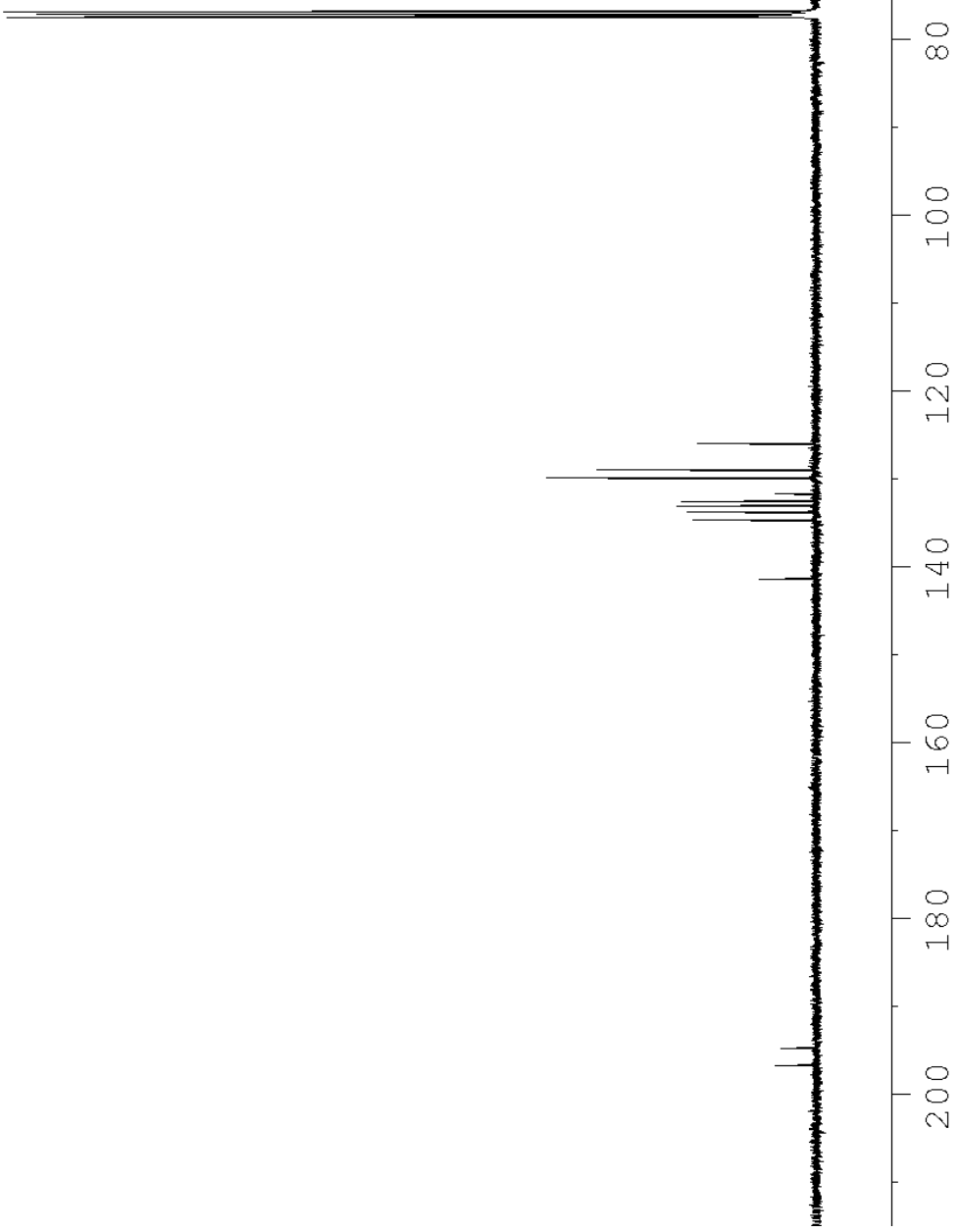



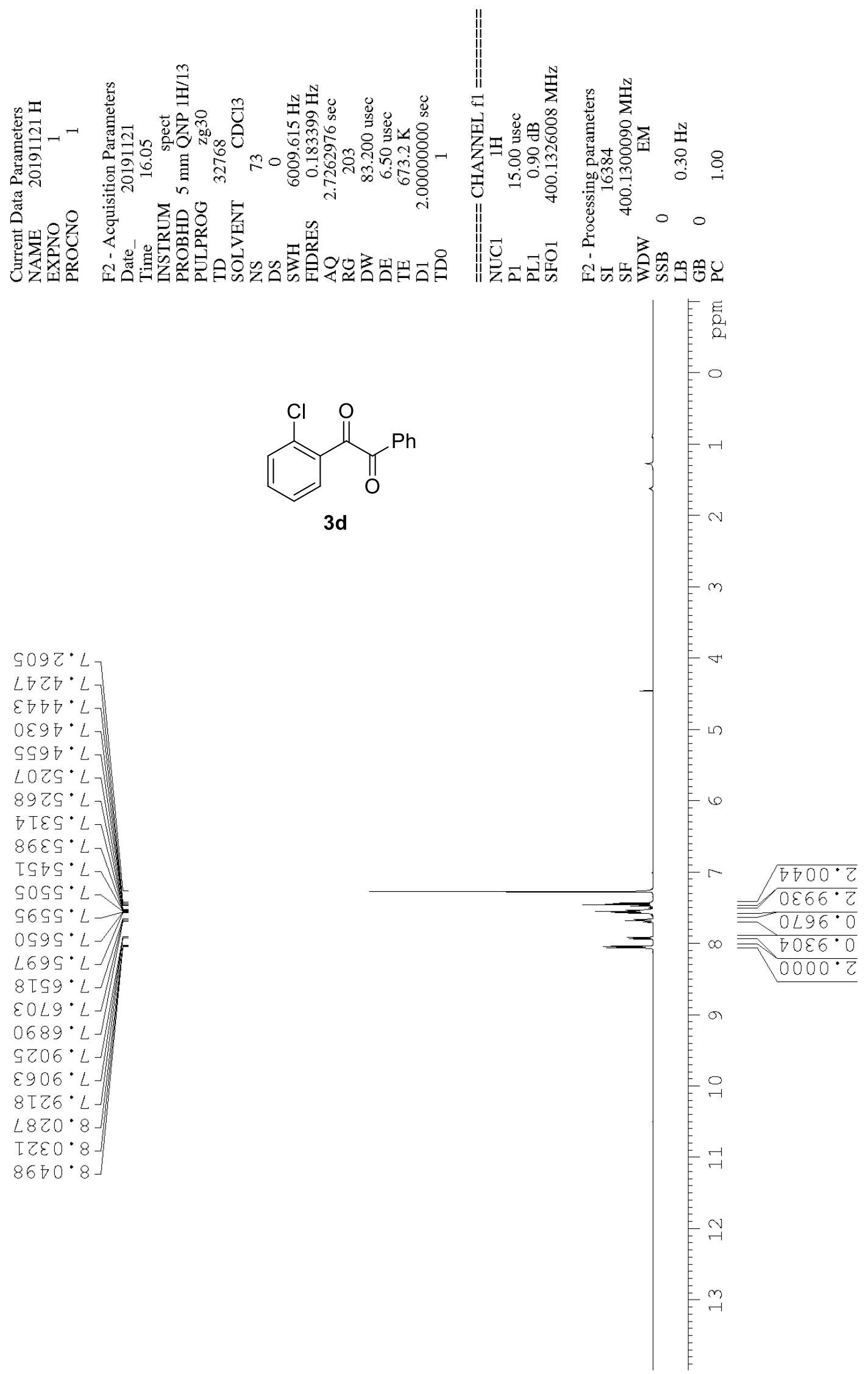<smiles>O=C(C(=O)c1ccccc1Cl)c1ccccc1</smiles> 

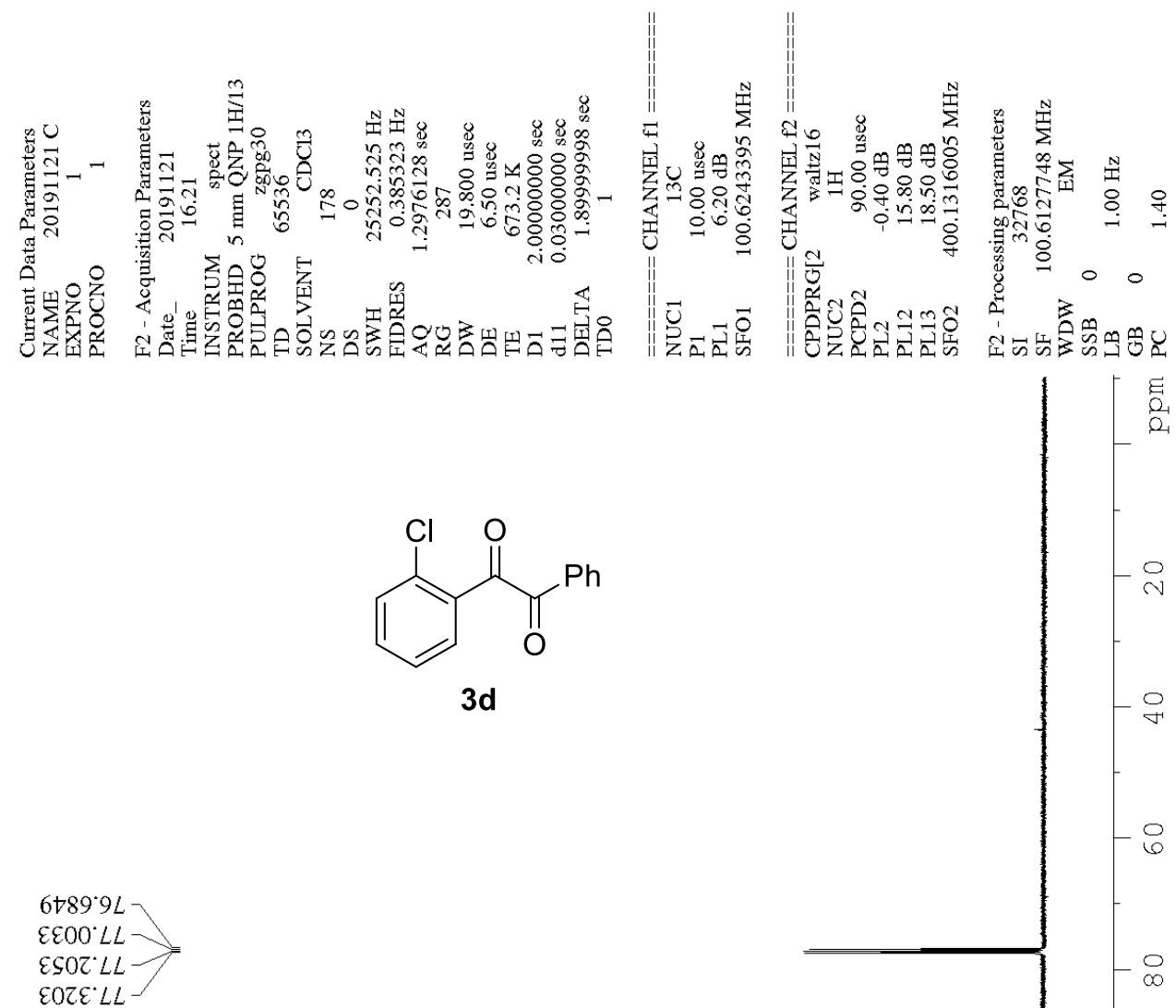<smiles>O=C(C(=O)c1ccccc1Cl)c1ccccc1</smiles>

3d
$68 \subseteq \varepsilon^{\circ} \angle Z I$

E6L8 $8 \mathrm{ZI}$

IOOZ $0 \varepsilon \mathrm{I}$

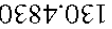

†00I $\tau \varepsilon \mathrm{I}$

ح99\& $\tau \varepsilon I$

$\varepsilon 908^{\circ} \varepsilon \varepsilon \mathrm{I}$

ऽ $896^{\circ} \varepsilon \varepsilon \mathrm{I}$

ऽ ¿ऽ $\nabla \varepsilon \mathrm{I}$

086 $\varsigma^{\circ} \nabla \varepsilon \mathrm{I}$

$\angle E+0{ }^{\circ}$ 26I

$6 \varepsilon \angle 9^{\circ} \varepsilon 6 \mathrm{I}-$

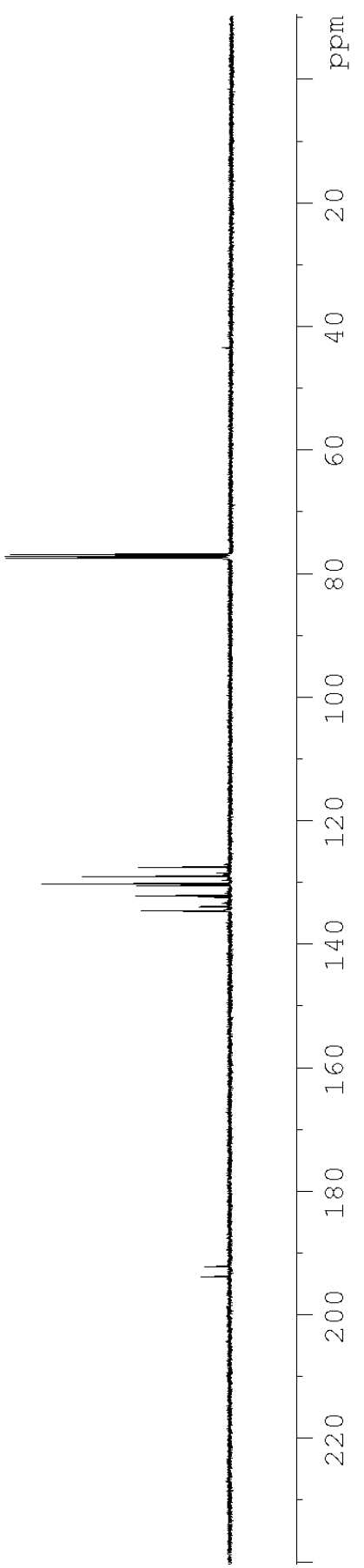




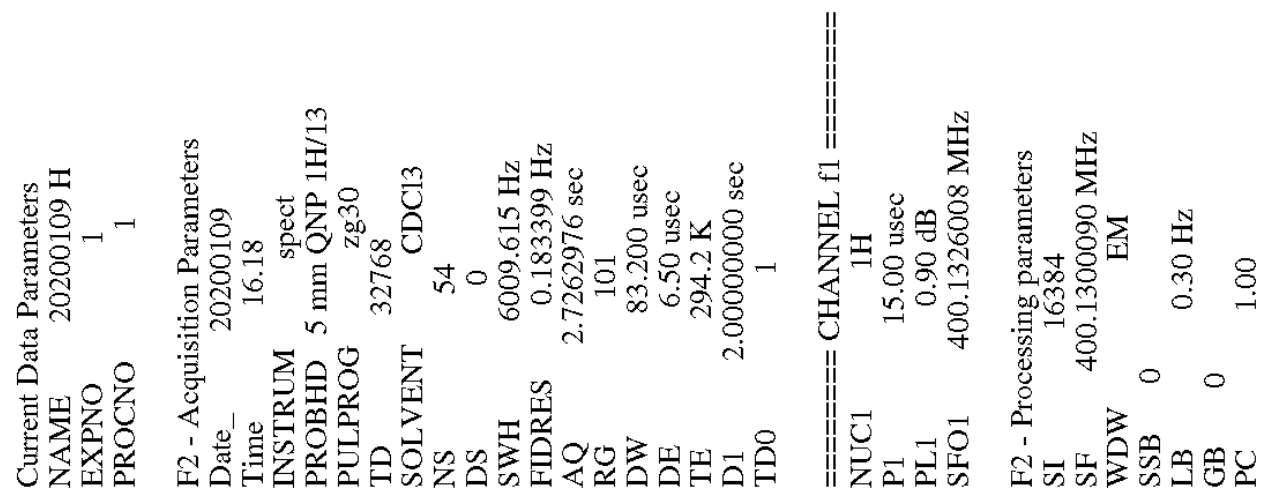

Eoot 2

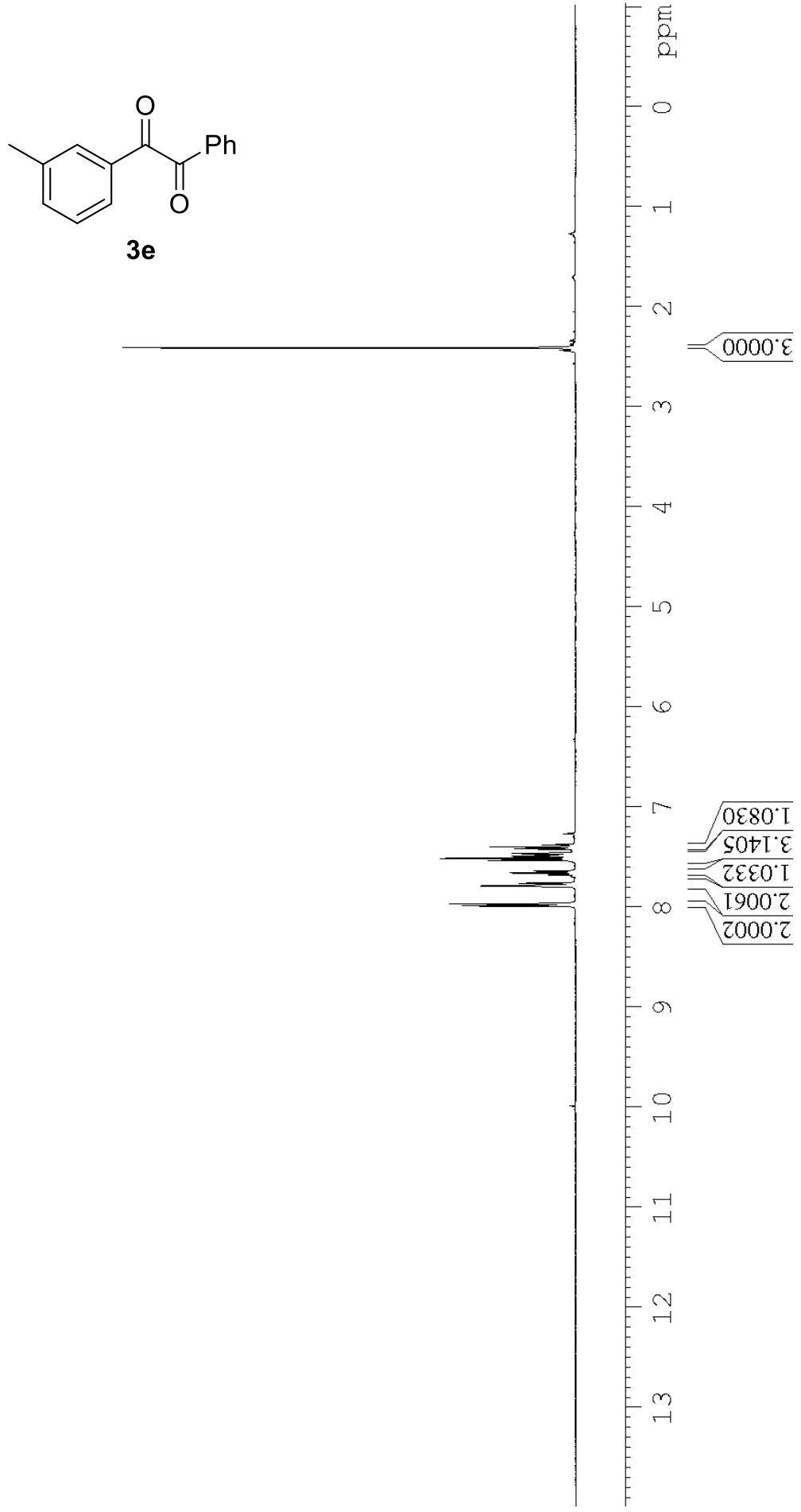

SO9Z: $L$

SZLEL

$\downarrow T 6 \varepsilon L$

toIt $L$

$\nabla L S+\angle$

$S 9 L t \cdot L$

$8 L 8 D^{\circ} L$

OLOS: $L$

$992 S^{\circ} L$

$\varepsilon \nabla \varepsilon 9^{\circ} L$

$8259^{\circ} \mathrm{L}$

tT $\angle 9^{\circ} L$

$\varepsilon 9 S L L$

II $8 L^{\circ} \mathrm{L}$

$\tau \varepsilon 8 L L$

I6S6 $\mathrm{L}$

$2796^{\circ} \mathrm{L}$

$2086^{\circ} \mathrm{L}$ 


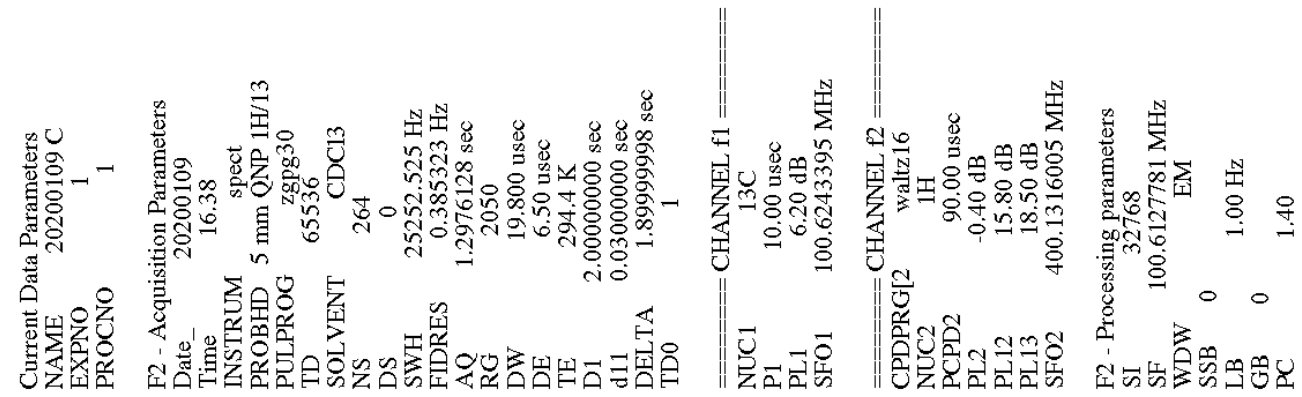

$860 Z \cdot I Z$

9L89.9L

I $\$ 00^{\circ} \mathrm{LL}$

EEZE $L L$<smiles>Cc1cccc(C(=O)C(=O)c2ccccc2)c1</smiles>

$3 e$
t8SI' $\angle Z$

L898.8ZI

ऽ\$\$6 $8 \mathrm{ZI}$

ttE8'6ZI

$60 t I^{\circ} 0 \varepsilon \mathrm{I}$

6IE6 ZEI

68ZL'SEI

Z8t6 8 EI

$\varepsilon 9 \angle 9^{\circ}+6 \mathrm{I}-$

$9028^{\circ}+6 \mathrm{I}>$

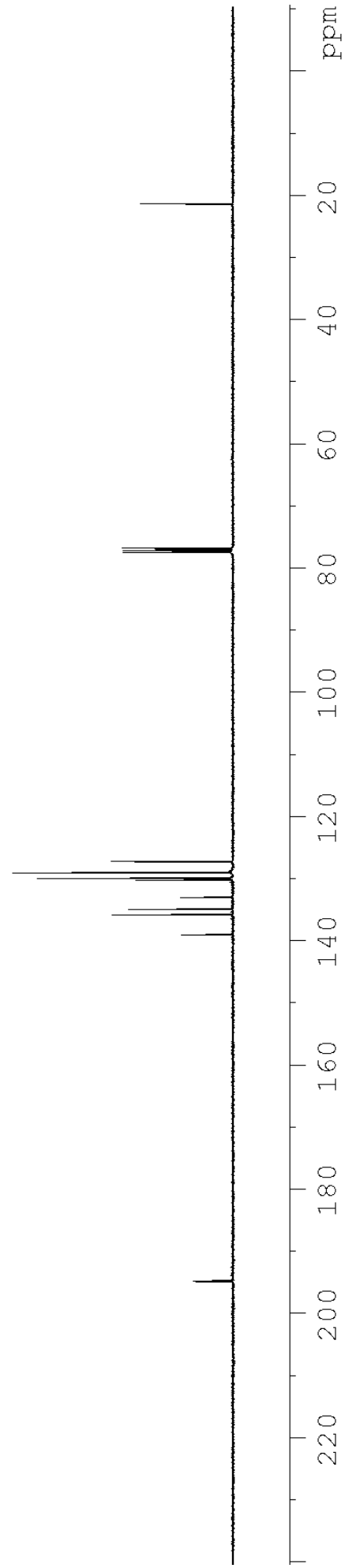



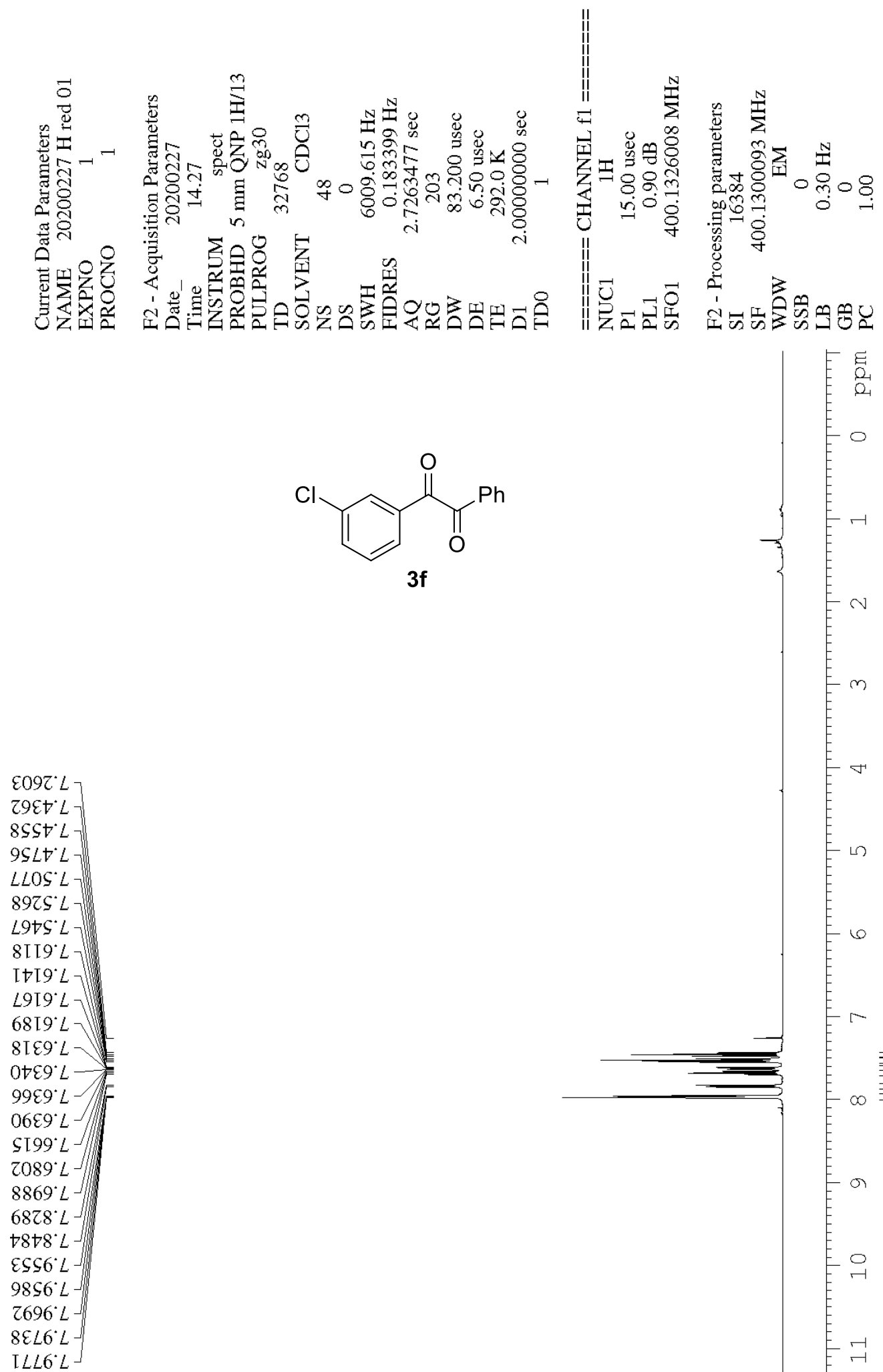<smiles>O=C(C(=O)c1cccc(Cl)c1)c1ccccc1</smiles>

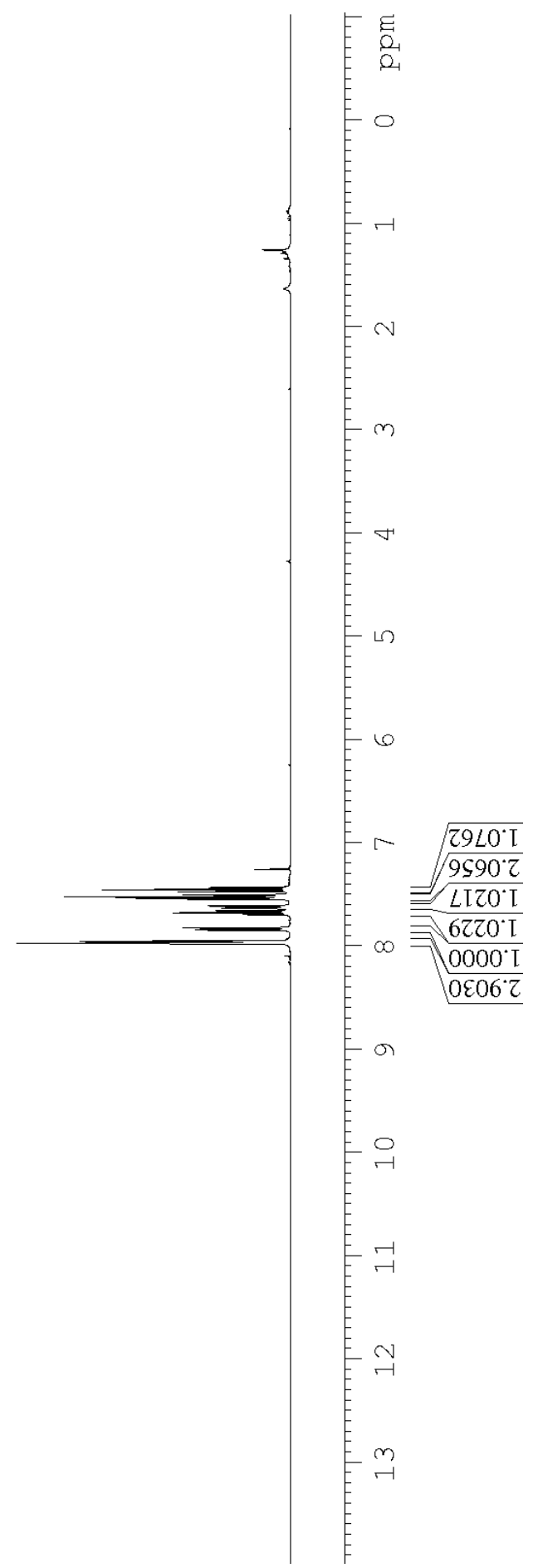



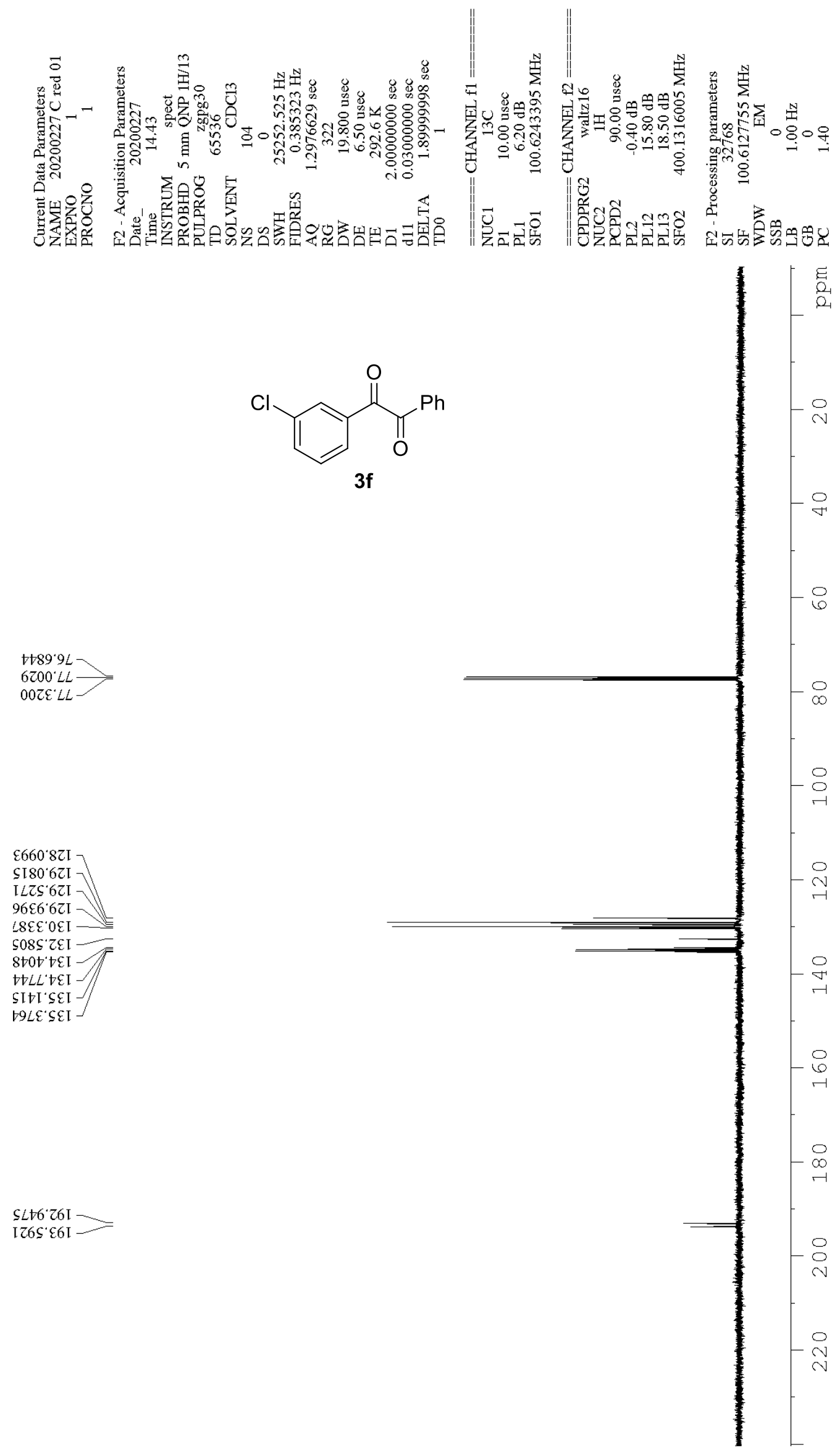


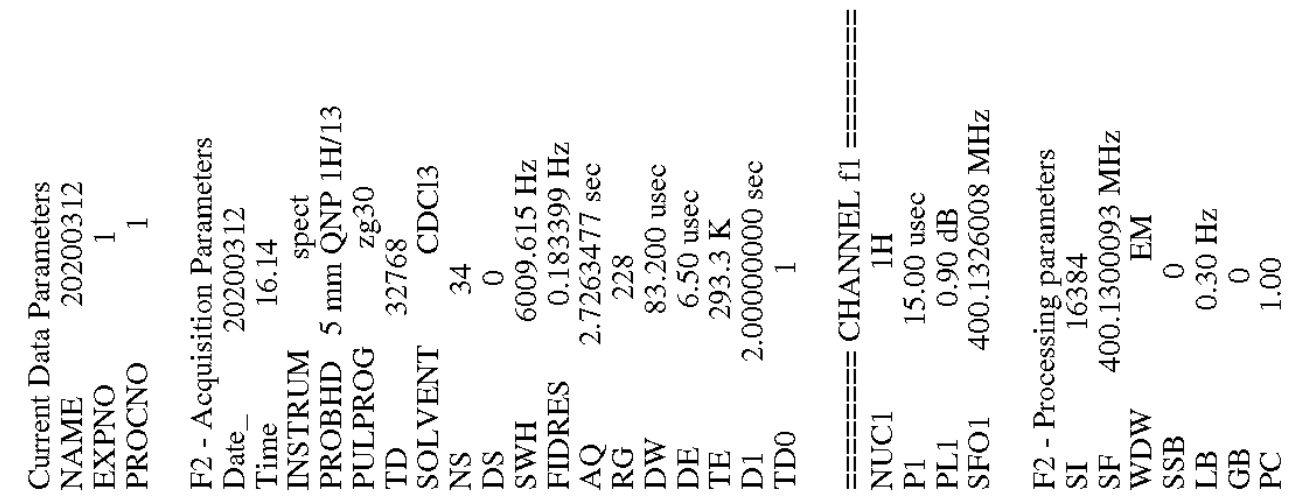<smiles>COc1cccc(C(=O)C(=O)c2ccccc2)c1</smiles>

$3 g$
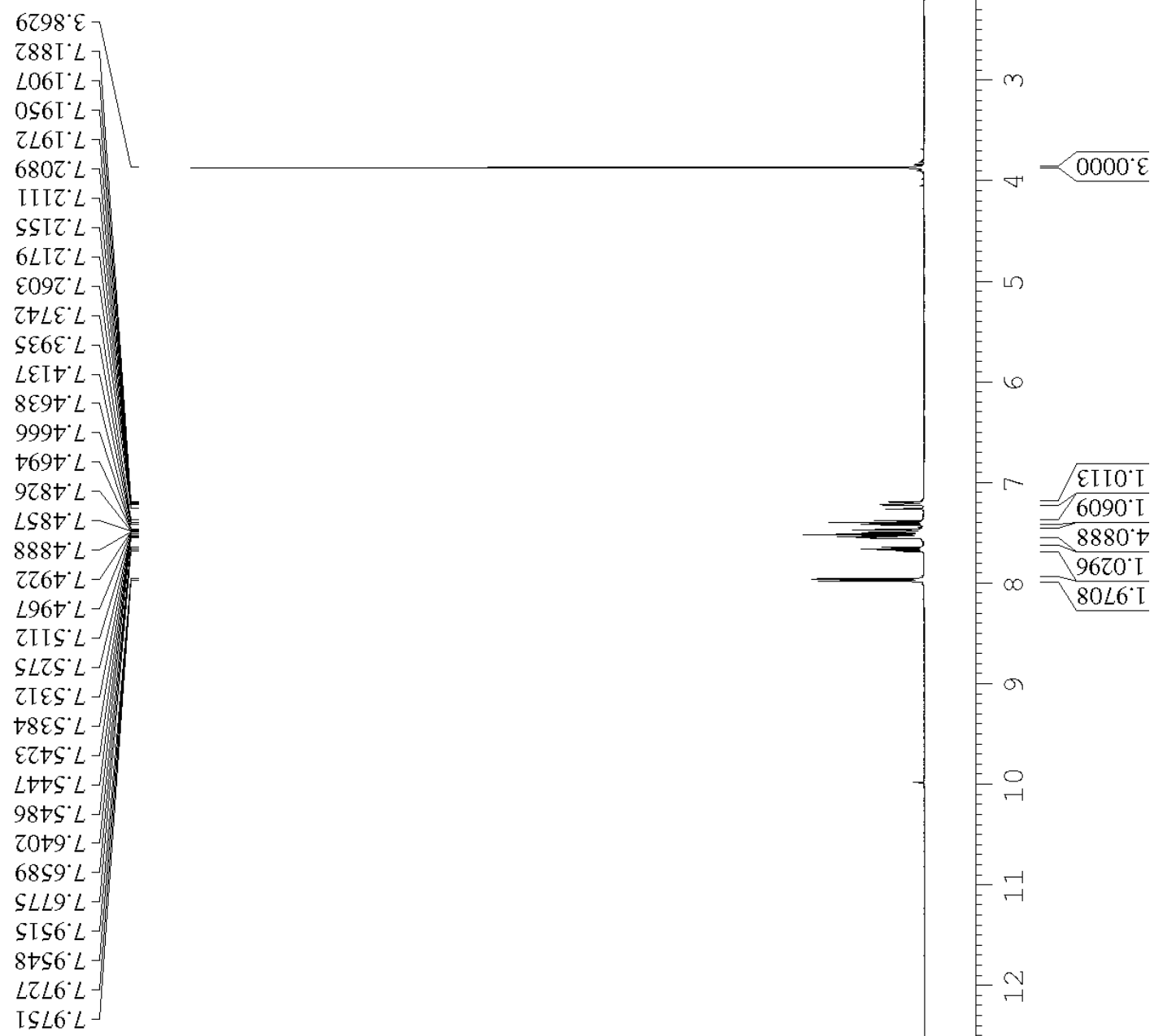


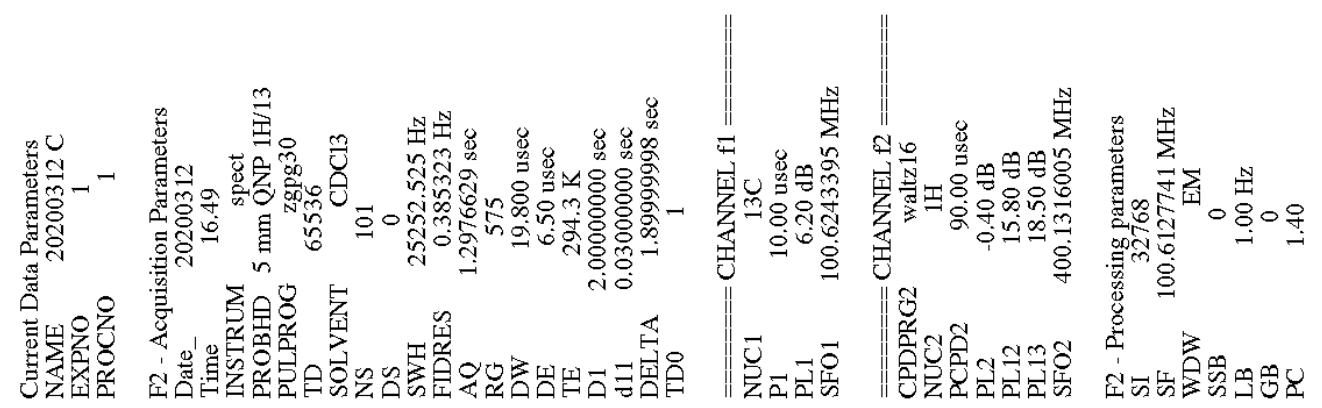<smiles>COc1cccc(C(=O)C(=O)c2ccccc2)c1</smiles>

3g

'SIS'SS

$0689^{\circ} 9 \mathrm{~L}$

$0900^{\circ}$

$\varepsilon+Z \varepsilon \cdot L L$

OESL'ZII -

ऽZ06.IZI

$80 Z Z \cdot E Z I$

$\angle 266{ }^{\circ} 8 \mathrm{II}$

SOL8.6ZI

$8620^{\circ} 0 \mathrm{EI}-$

S†Z6'ZEI

806I' $\downarrow \mathcal{E} \mathrm{I}$

$\varepsilon 6 \angle 8^{\prime} \downarrow \varepsilon 1-$

I0t0.09I

$0 \mathrm{~L} L \mathrm{t}^{\circ} t 6 \mathrm{I} \longrightarrow$

$0+6 t^{\circ}+6 \mathrm{I} \longrightarrow$

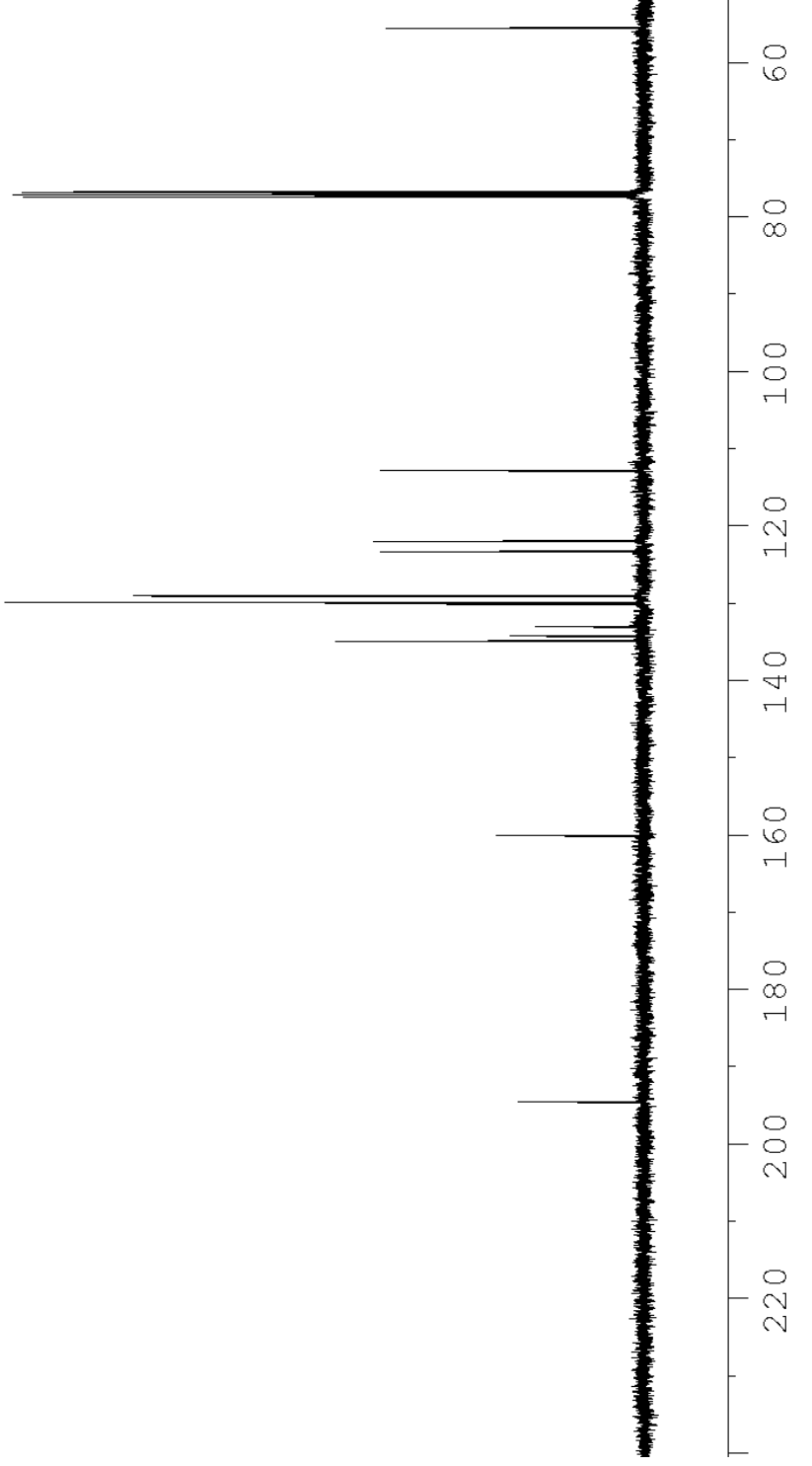




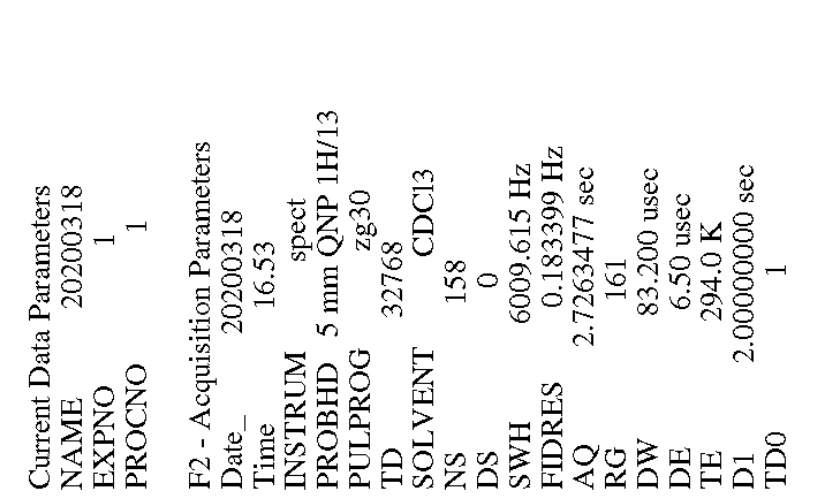

$\begin{array}{ll}\| & \\ \|\end{array}$

$2092 L$

$8 E Z S^{\circ} L$

IEtS $L$

$629 S^{\circ} L$

9L $89^{\circ} \mathrm{L}-$

$\varepsilon+89^{\circ} \mathrm{L}$

$\nabla \angle 89^{\circ} L$

IEO $L L^{\circ} L$

$602 L^{\circ} L$

$S 0 t L L$

$S 09 L^{\circ} L$

$\angle 086^{\circ} \mathrm{L}-$

$0+866^{\circ} \mathrm{L}$

$0200{ }^{\circ}$

$8 S 6 Z^{\circ} 8$

$8862^{\circ} 8$

8IOE: 8

ISIE: 8

Z8I $\varepsilon^{\circ} 8$

IIZE:8

Sโ $87^{\circ} 8$

$8 \varepsilon 8 t^{\circ} 8$

$0487^{\circ} 8$

七68 $\mathrm{t}^{\circ} 8$

6LOS: 8

七tOS 8

$\neg \operatorname{LOS}^{\circ} 8$

$8605^{\circ} 8$

I66 L 8 -

$9 \varepsilon 08^{\circ} 8-$<smiles>O=C(C(=O)c1cccc([N+](=O)[O-])c1)c1ccccc1</smiles>

3h

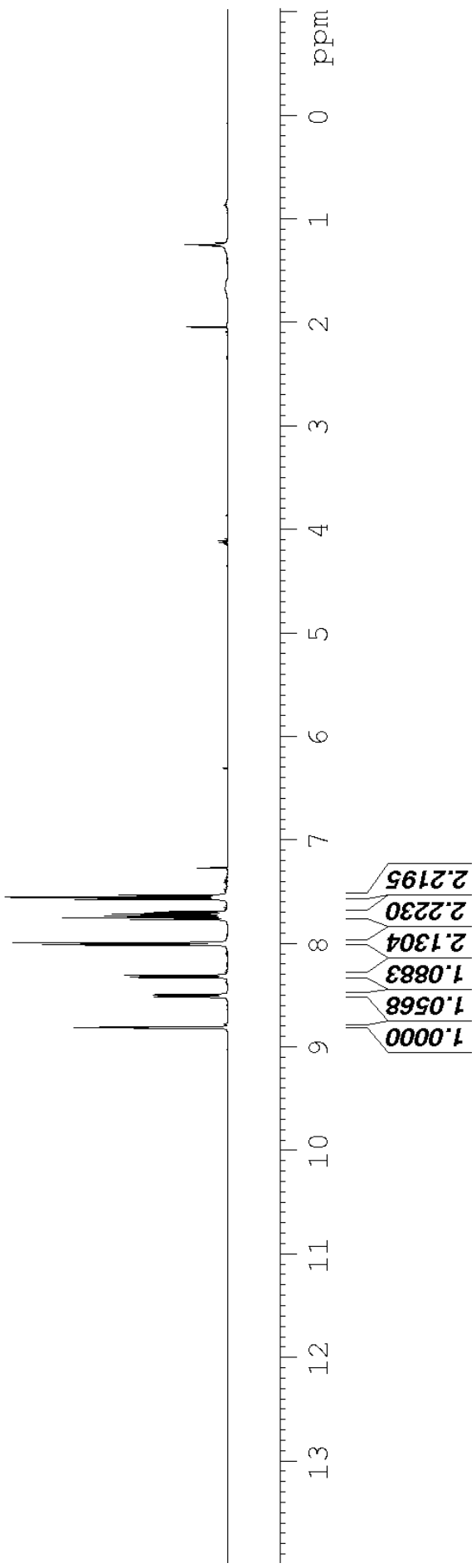



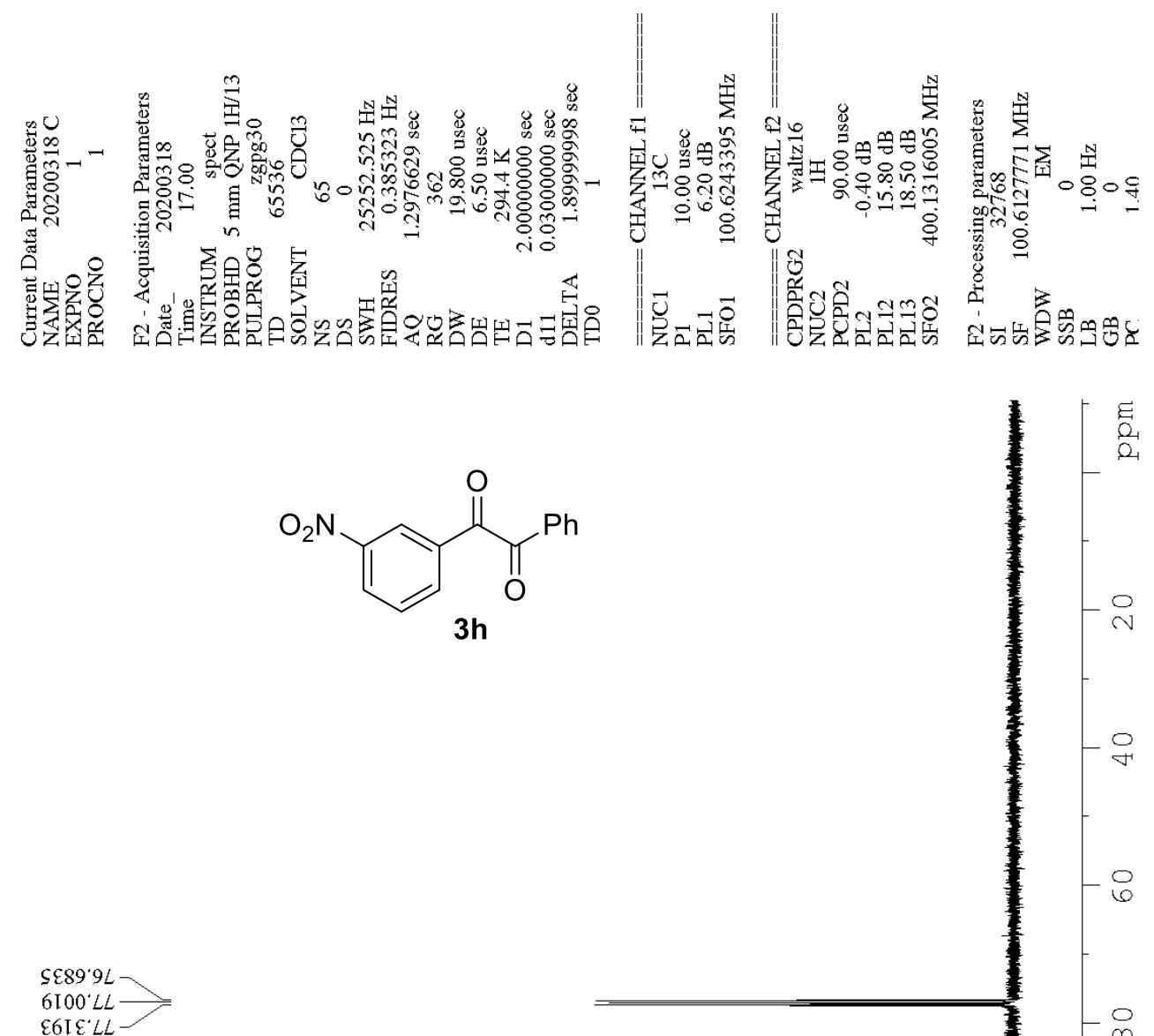<smiles>O=C(C(=O)c1cccc([N+](=O)[O-])c1)c1ccccc1</smiles>

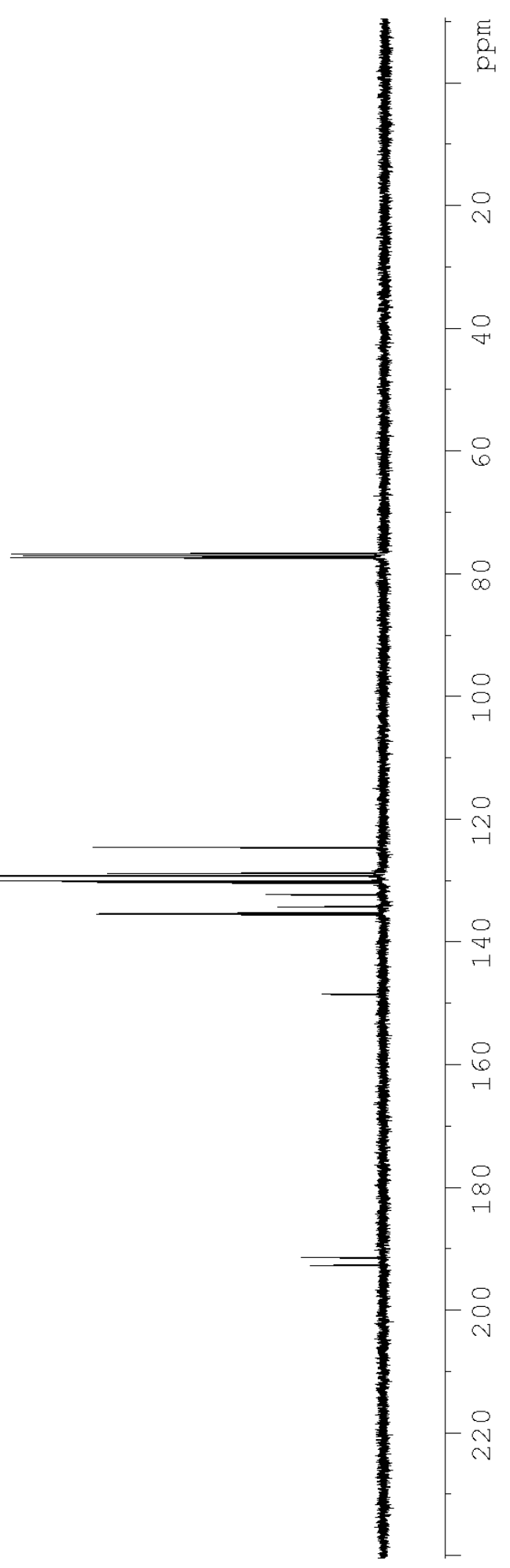

0L6E' I6I -

6ย I9. 26I - 


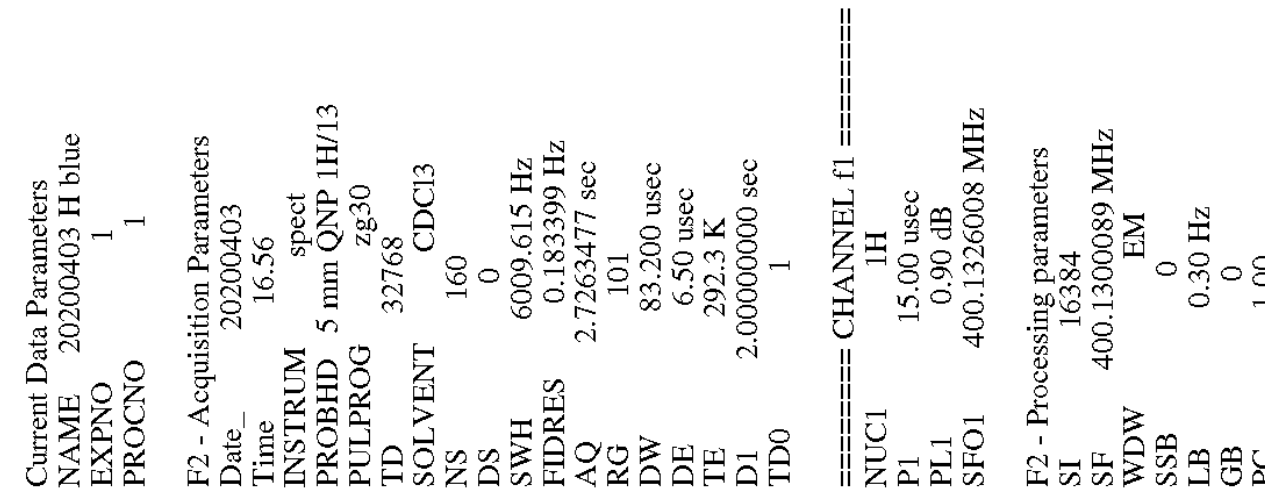<smiles>O=C(C(=O)c1ccc(-c2ccccc2)cc1)c1ccccc1</smiles>

$3 \mathbf{i}$

S092

$\$ 90 t^{\circ} L$

$8+C t{ }^{\circ} L$

$L Z t t^{\circ} L$

ZL9t L

$6 \varepsilon 0 S L$

$S E I S^{\circ} L-$

$8 Z \varepsilon S^{\circ} L$

ZZSS $L$

$\angle 929^{\circ} \mathrm{L}$

$8+79^{\circ} \mathrm{L}$

I9S9 $\mathrm{L}$

$6+\angle 9^{\circ} \mathrm{L}$

$\nabla \varepsilon 69^{\circ} \mathrm{L}-$

$\varepsilon 62 L L$

IOSLL

ESL0 8

$\angle \varepsilon \varepsilon 0{ }^{\circ} 8$

2950 8 -

[ $\angle L 0^{\circ} 8$

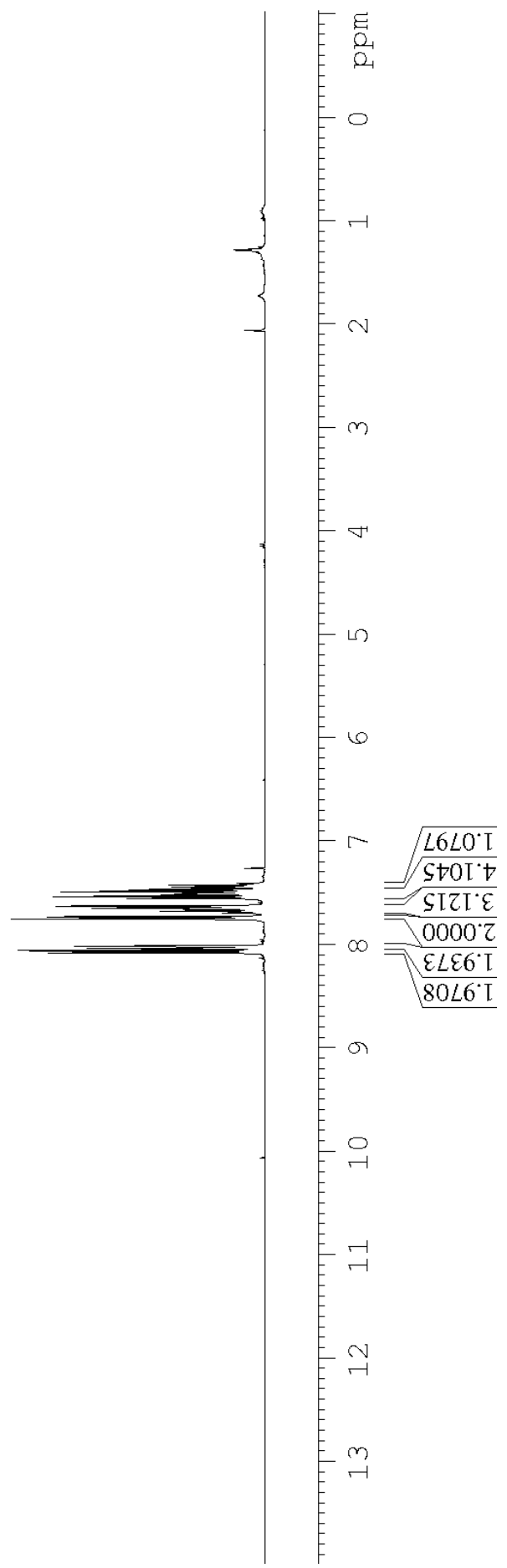




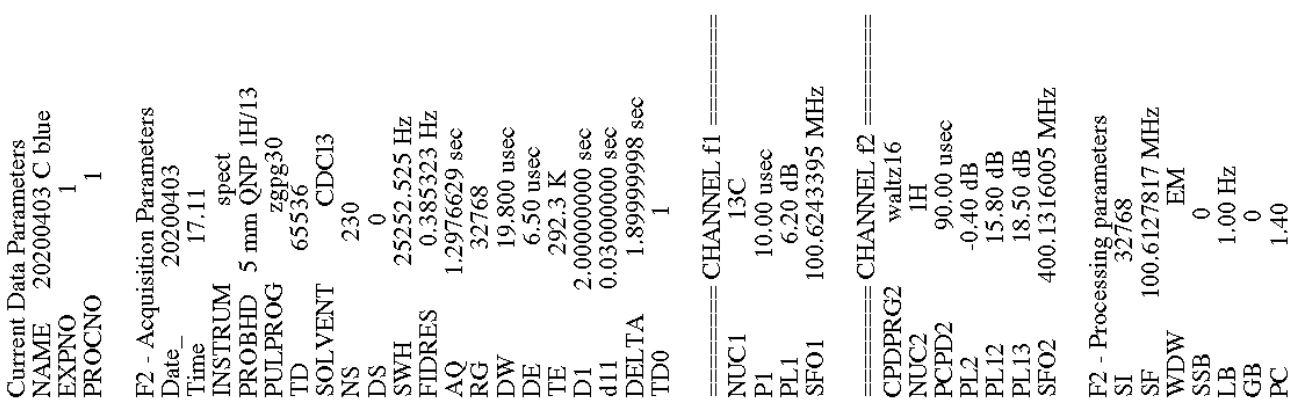

$8+89^{\circ} 9 \mathrm{~L}$

$\triangle \varepsilon 00^{\circ} \mathrm{LL}$

LOZE LL -<smiles>O=C(C(=O)c1ccc(-c2ccccc2)cc1)c1ccccc1</smiles>

$3 \mathbf{i}$

8I8Z: LZI

$508 \mathrm{~S}^{\circ} \mathrm{LZI}$

I685'8ZI -

86L6 $8 \mathrm{ZI}$

†8L8.6ZI

$\angle 977^{\circ} 0 E \mathrm{I}$

Z99S'IEI

SL06'ZEI

I I98. $\downarrow$ EI

$5 \mathcal{E S E} 6 \mathcal{E L}$

96IS $L D I$

$9860^{\circ} \mathrm{t} 6 \mathrm{I}-$

80 IS' $^{\circ} 6 \mathrm{I} \longrightarrow$

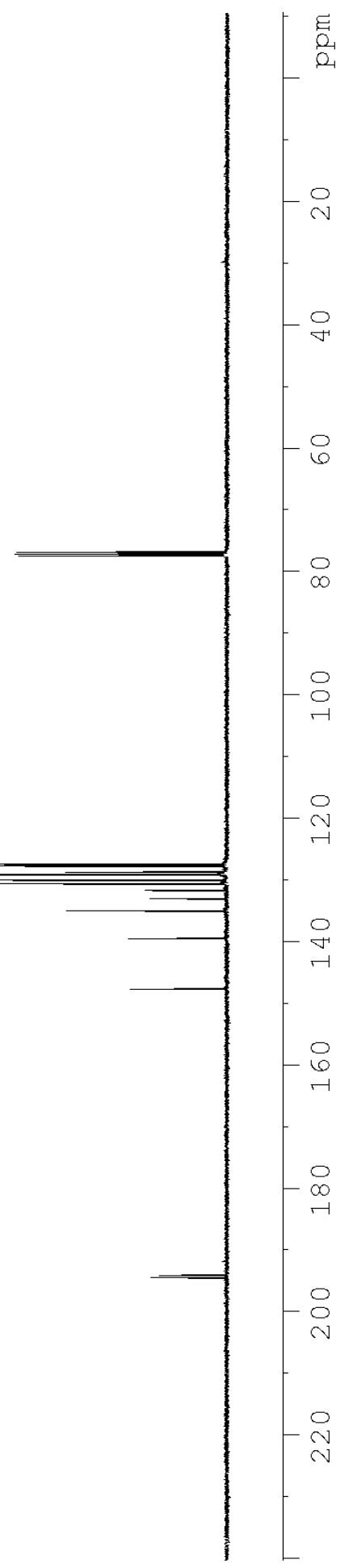




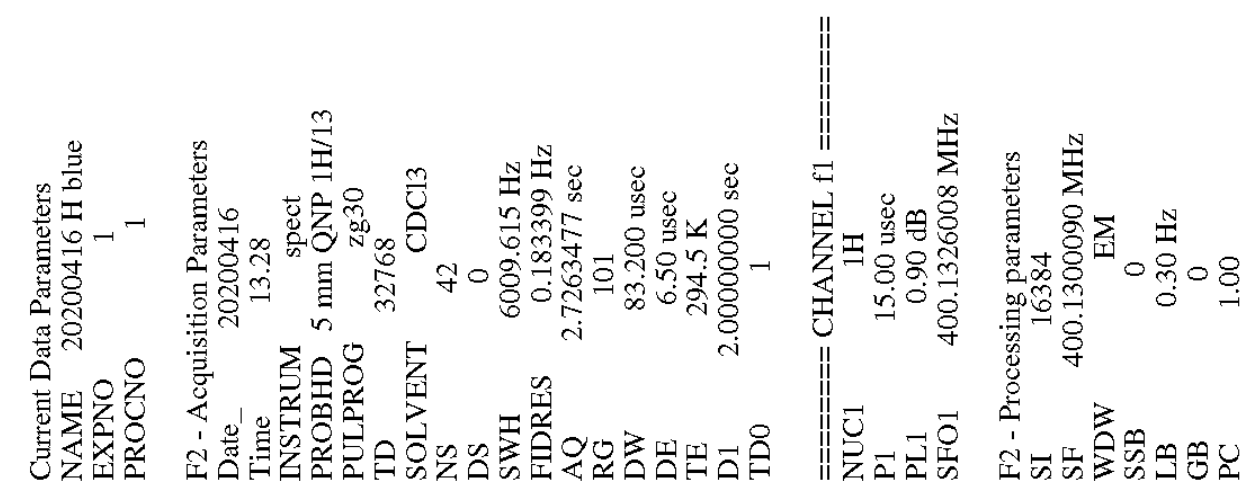<smiles>Cc1ccc(C(=O)C(=O)c2ccccc2)cc1</smiles>

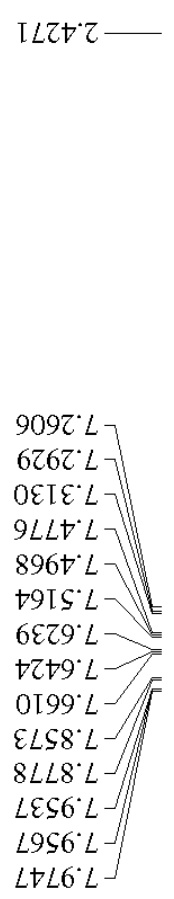

3j

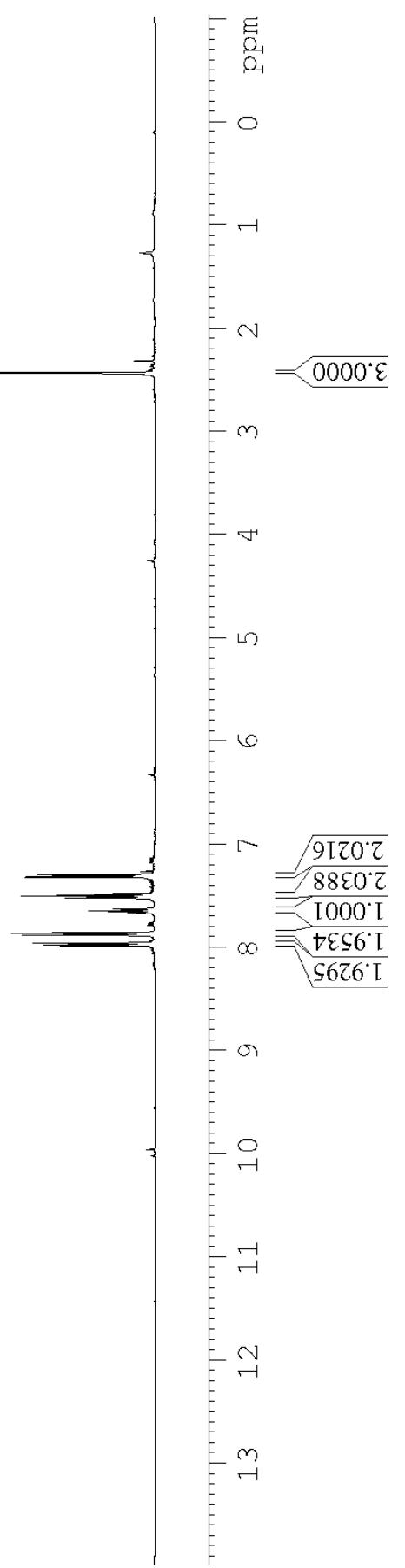




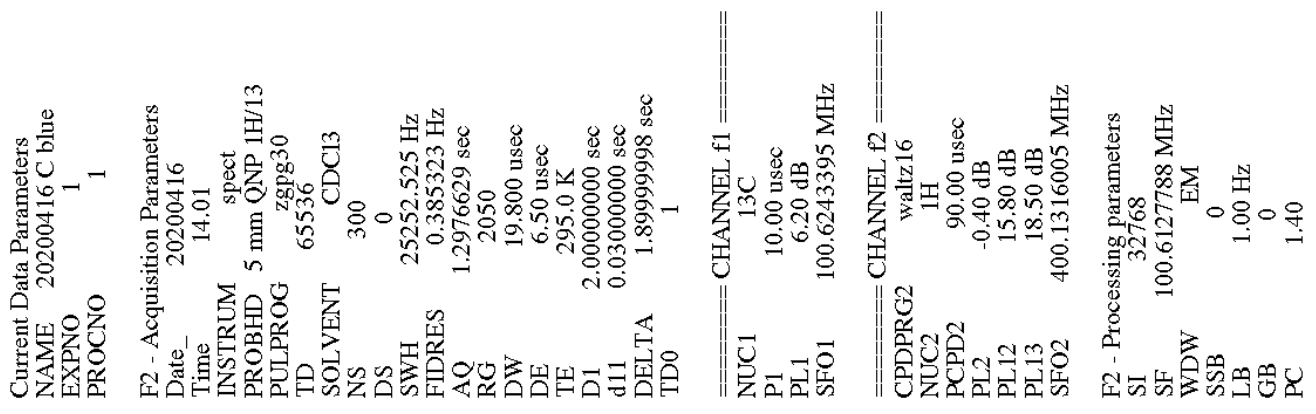

$6298^{\circ} I Z$<smiles>Cc1ccc(C(=O)C(=O)c2ccccc2)cc1</smiles>

3j

$6989^{\circ} 9 \mathrm{~L}$

$\varepsilon 700^{\circ} \mathrm{LL}$

$\angle Z Z E ' L L$

E6I6.8ZI

ZL89.6ZI

$\rightarrow \angle 08^{\circ} 6 \mathrm{ZI}$

sSt6.6ZI

z885 0 \&I

$9686^{\circ} \mathrm{ZEI}$

$\varsigma \subset t L ' t \varepsilon I$

8 S $8 I^{\circ} 9 t \mathrm{I}$

$0+9 Z \div 6 \mathrm{I} \longrightarrow$
$+6 Z L+6 \mathrm{I}$

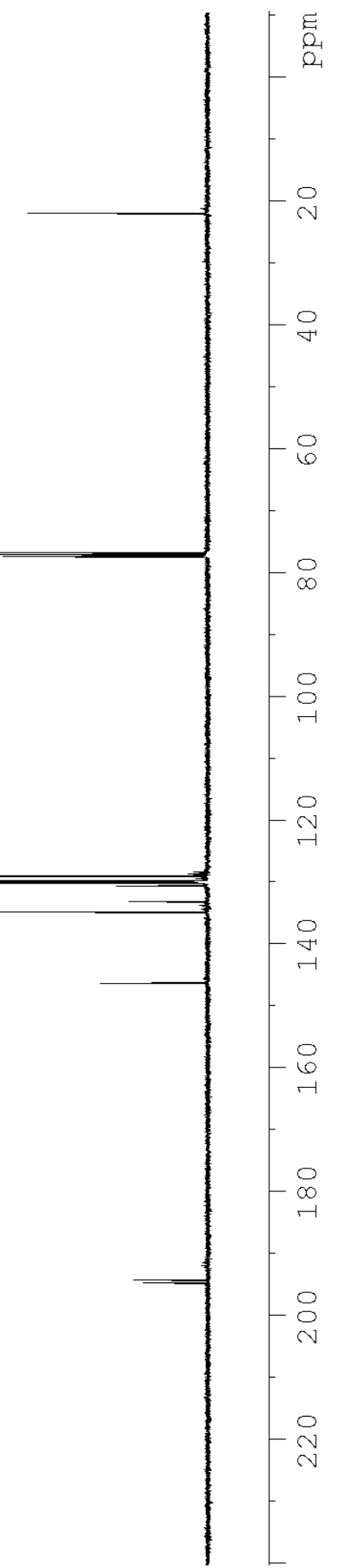




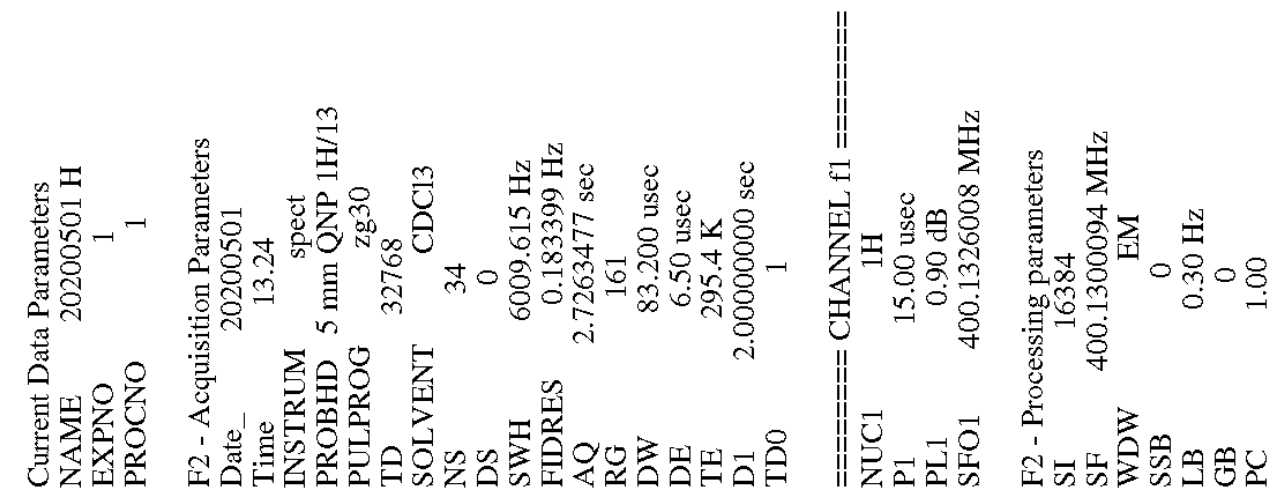

$86 S T^{\circ} L$

$0 \angle 9 D^{\circ} L$

$\nabla Z \angle D^{\circ} L$

$\nabla 9 L t^{\circ} L$

$6 \varepsilon 6 \nabla^{\circ} L$

EEIS $L$

6ZES $L$

$0979^{\circ} \mathrm{L}$

$9799^{\circ} \mathrm{L}$

IE89. L

ZE06 L T

$\angle 806^{\circ} \mathrm{L}-$

OEI $6^{\circ} \mathrm{L}-$

$8 S 26^{\circ} \mathrm{L}$

$20866^{\circ} \mathrm{L}$

$9 S E 6^{\circ} \mathrm{L}-$

$98+66^{\circ} \mathrm{L}$

$\angle I S 6^{\circ} L^{-}$

$8696^{\circ} \mathrm{L}$<smiles>O=C(C(=O)c1ccc(Cl)cc1)c1ccccc1</smiles>

3k 

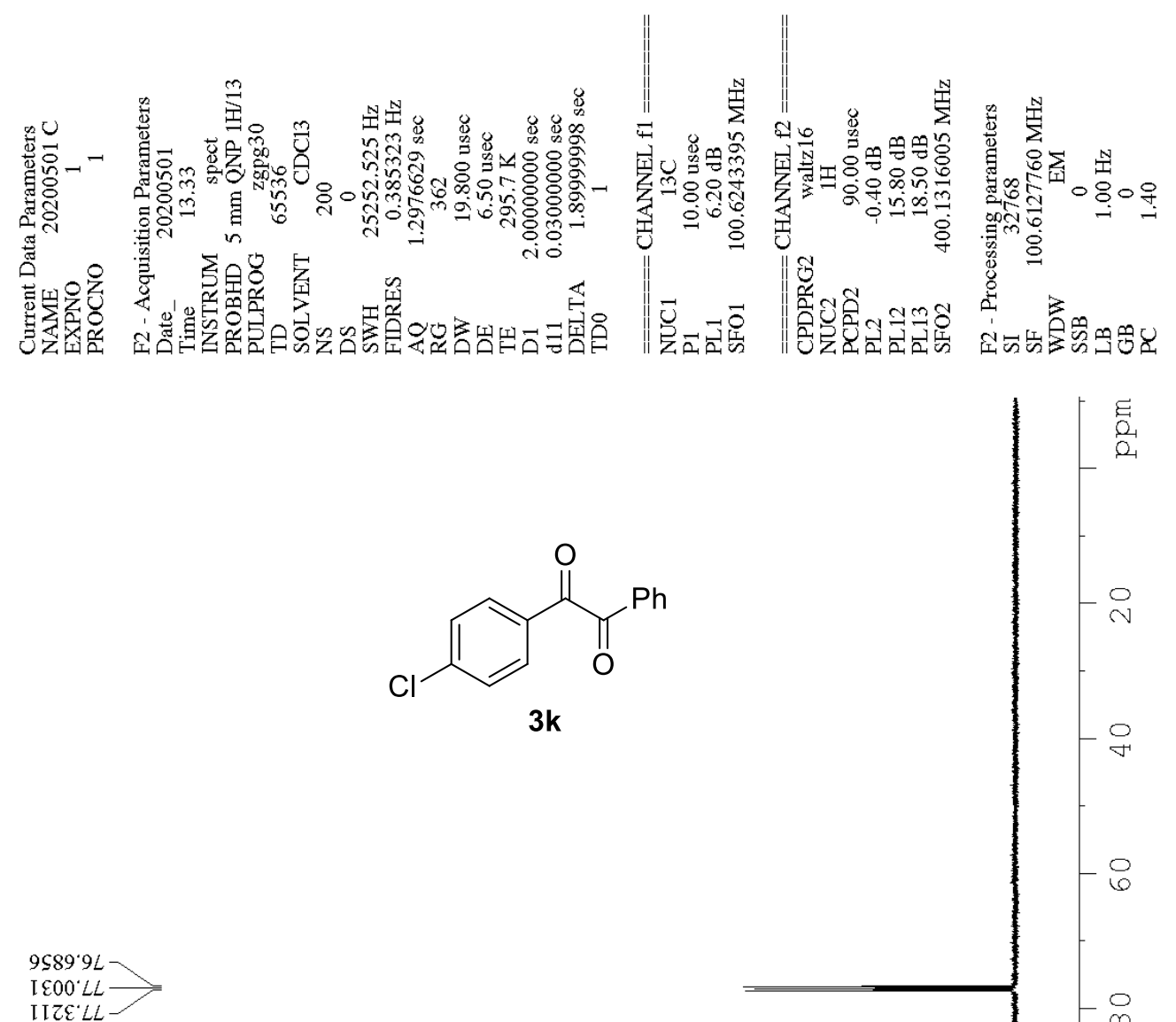<smiles>O=C(C(=O)c1ccc(Cl)cc1)c1ccccc1</smiles>

I $\angle E 0^{\circ} 6 \mathrm{ZI}$ $096 \varepsilon 62 \mathrm{I}$

8S68.6ZI

S9LI'IEI

6†LZ'IEI

Z†OL'ZEI

¿†EO $\subseteq \mathcal{}$ I

9LtS'ItI

$7920^{\circ} \varepsilon 6 \mathrm{I}-$

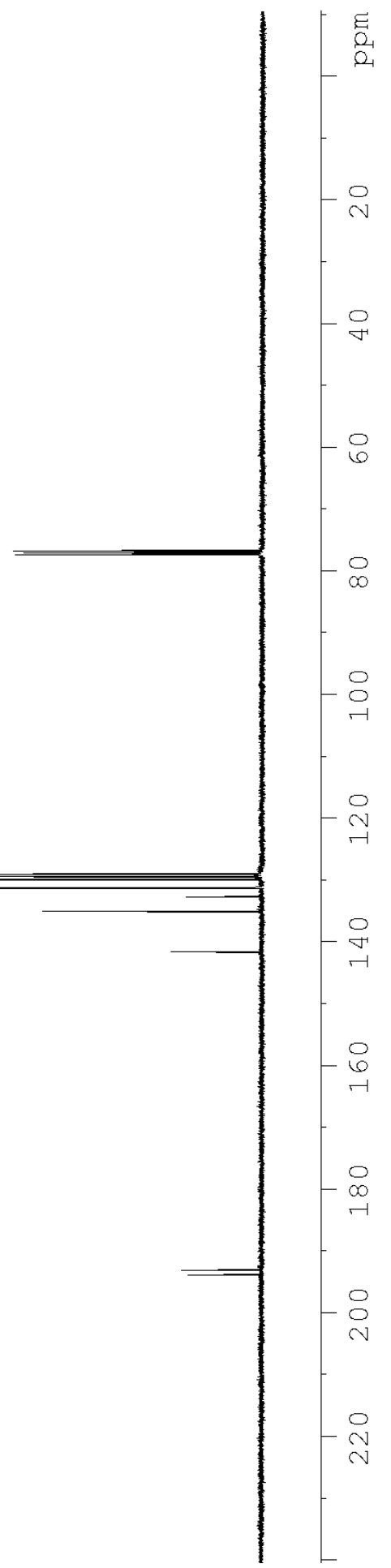




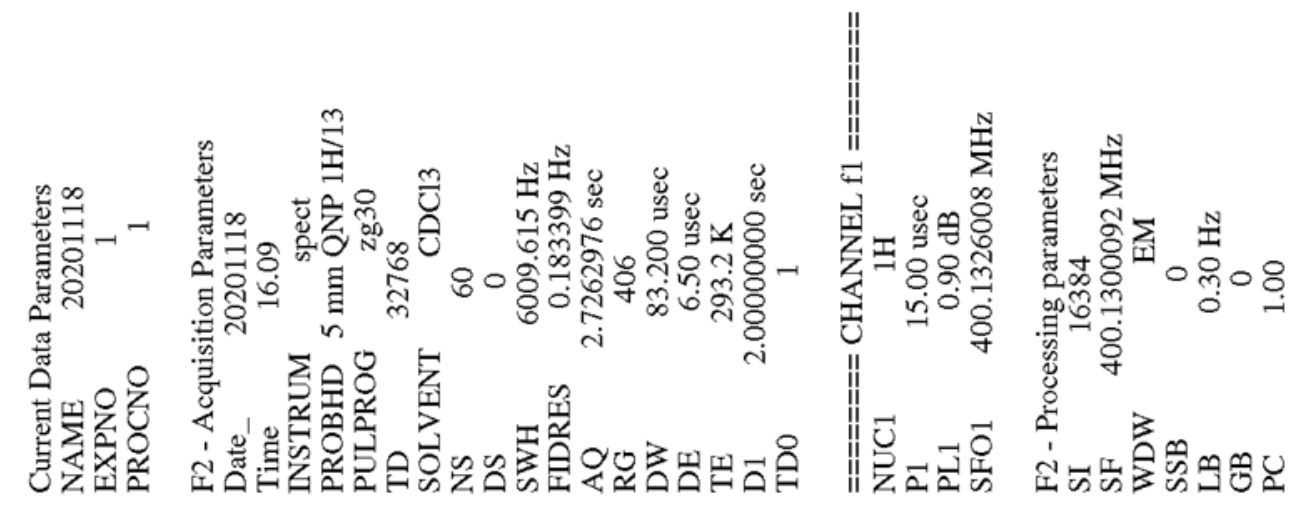<smiles>COc1ccc(C(=O)C(=O)c2ccccc2)cc1</smiles>
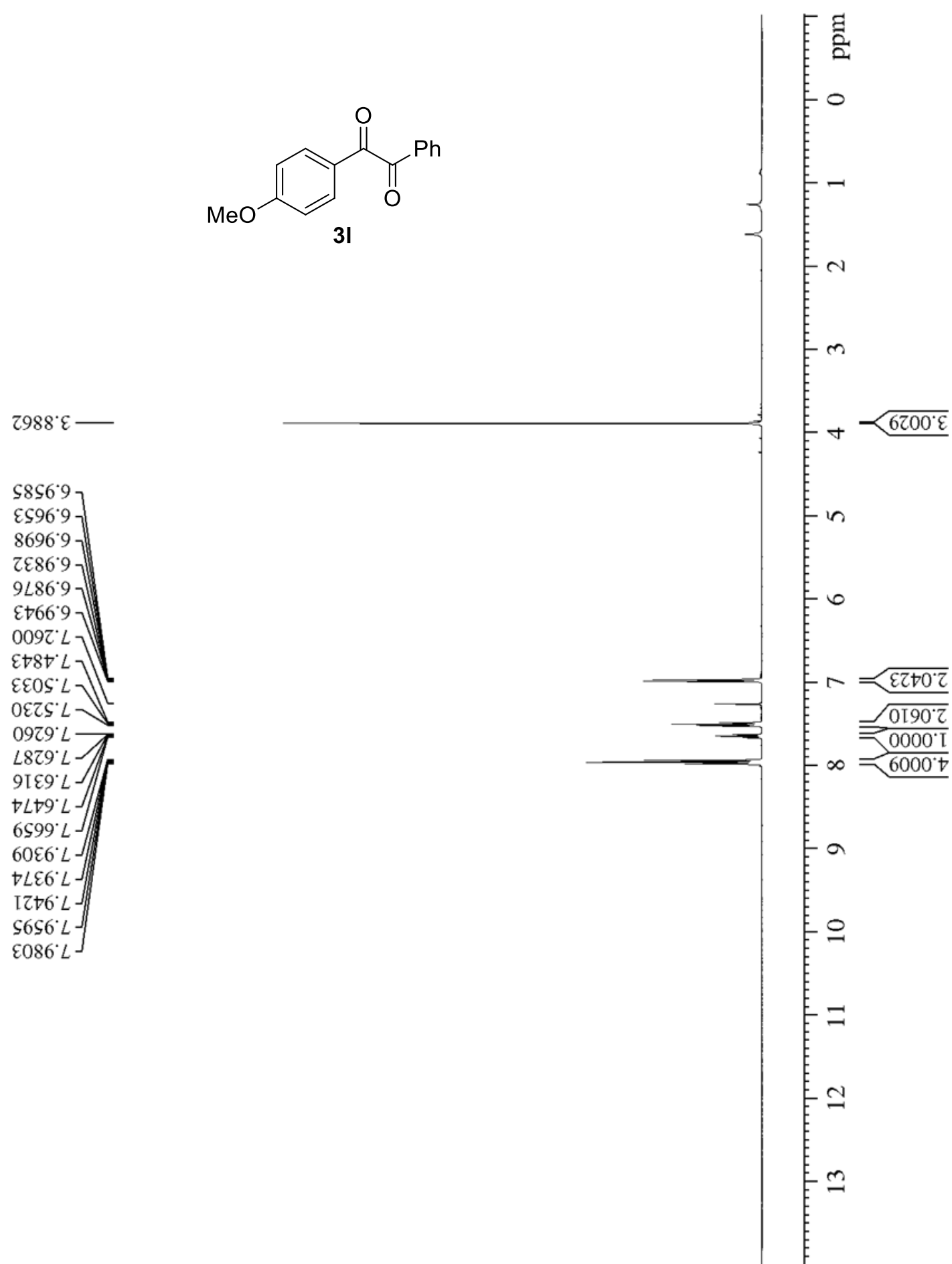


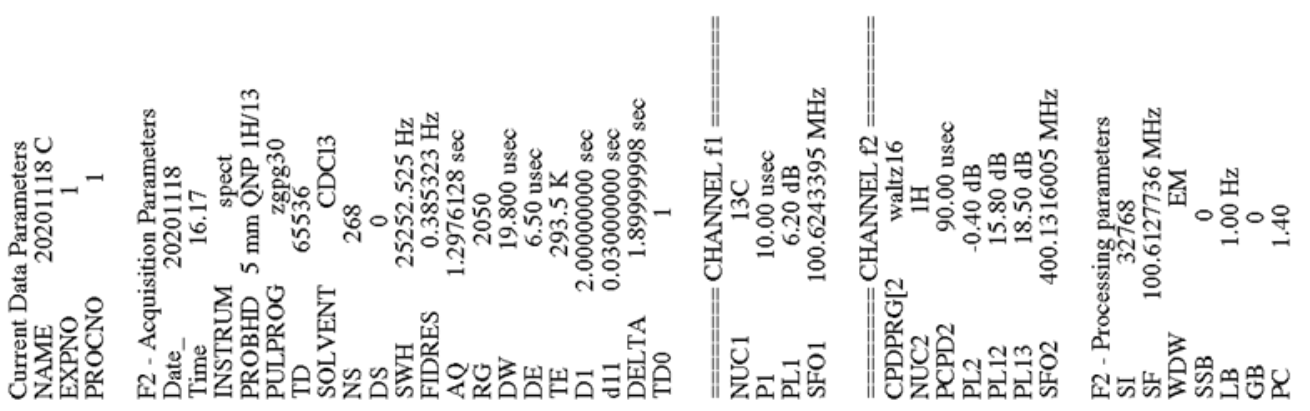

§6ع9 \$S<smiles>COc1ccc(C(=O)C(=O)c2ccccc2)cc1</smiles>

$8189^{\circ} 9 L$

$0000^{\circ} L L$

$0 L I \mathcal{E} L L$

3I

$88 \varepsilon \varepsilon^{\prime} \downarrow I \mathrm{II}$

ऽ\&્0 $9 Z \mathrm{I}$

z\&\&6 $8 \mathrm{ZI}$

9t88.6ZI

¿\&LE' $z \varepsilon$

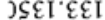

¿SIL'十EI

$9296^{\circ}$ t9I

$\angle 9 S I^{\circ} \varepsilon 6 \mathrm{I}-$

$8058^{\circ}+6 \mathrm{I}=$

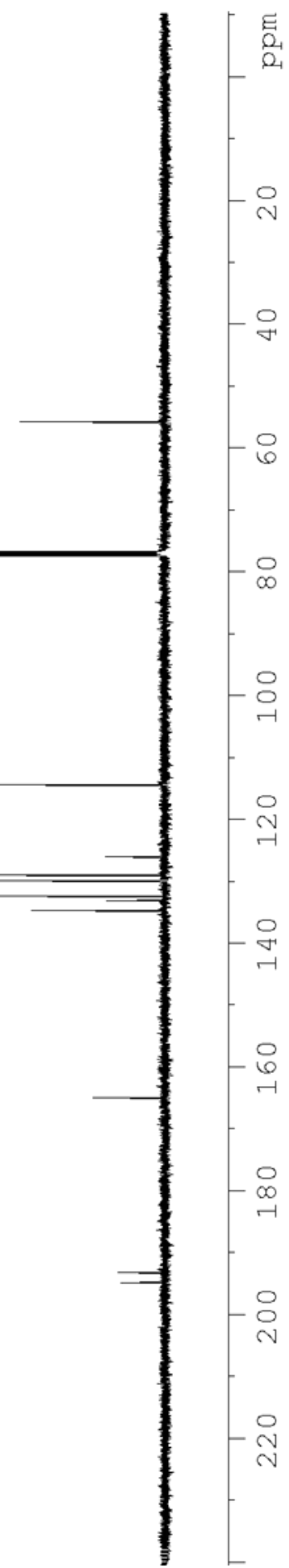




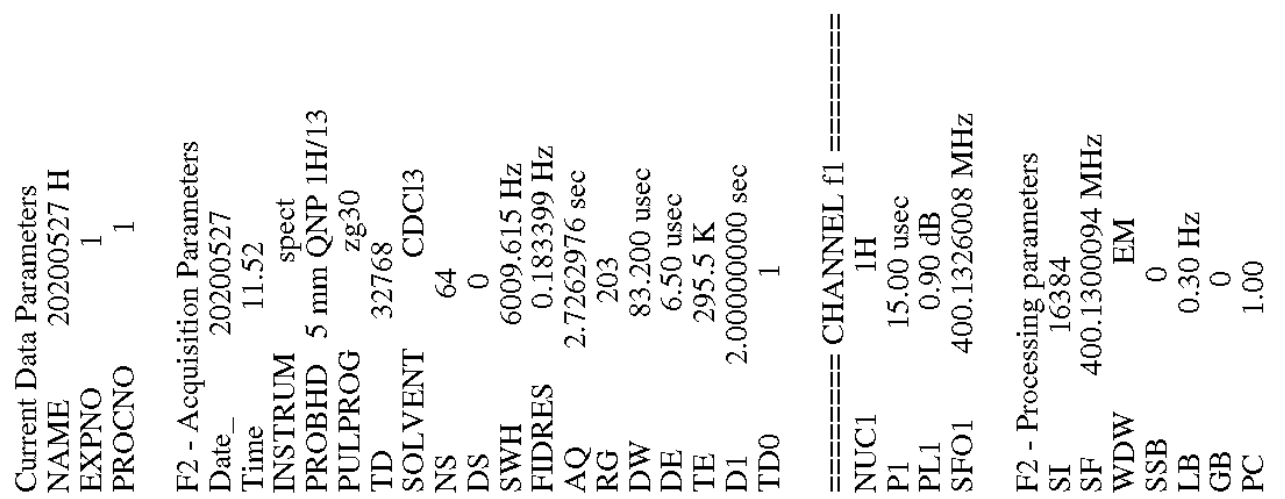

$0092: L$

ZSIS $L$

$S+E S L$

$\tau \nabla S S^{\circ} L$

$8 \angle 99^{\circ} L$

$\angle 0 \angle 9^{\circ} L$

$\varepsilon 689^{\circ} \mathrm{L}$

ZSOLL L

$8 \angle 0 L L$

$90 L L L$

ZI6LL

ZL96 L

$S 0 \angle 6^{\circ} L$

$7886^{\circ} \mathrm{L}$

$\mathrm{S} 066^{\circ} \mathrm{L}$

IS60 8<smiles>O=C(C(=O)c1ccc(C(F)(F)F)cc1)c1ccccc1</smiles>

SSI I. 8

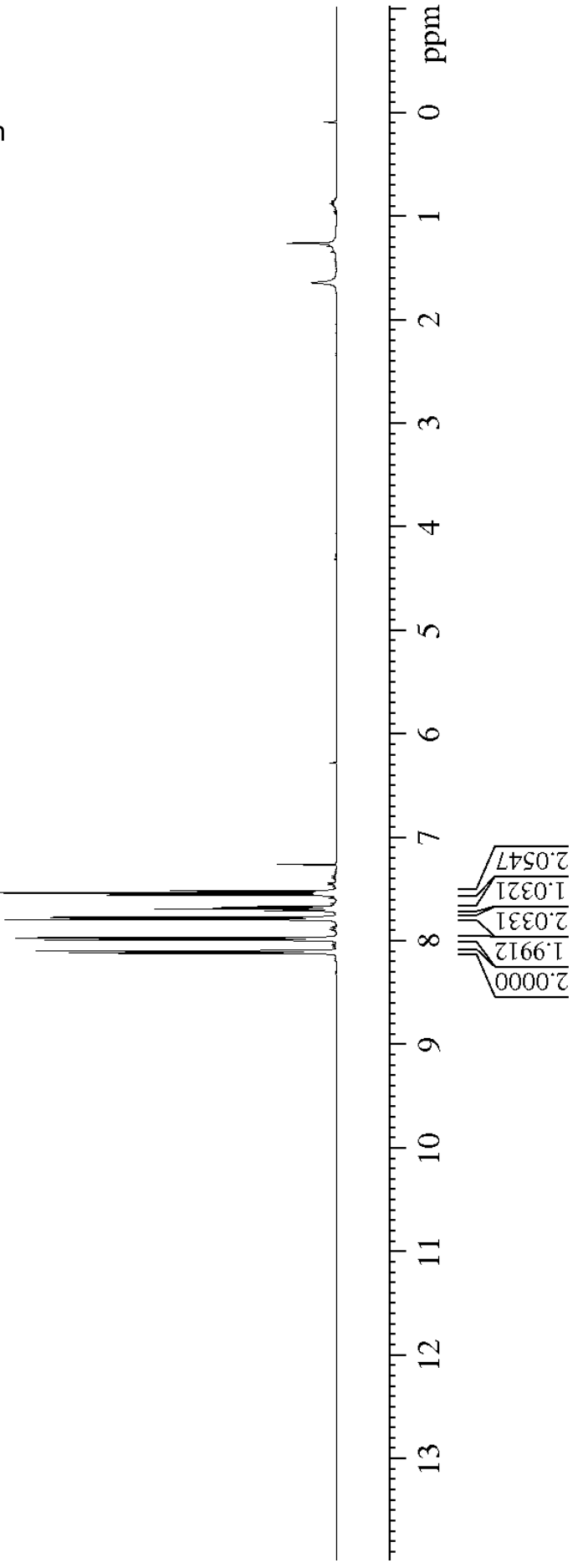




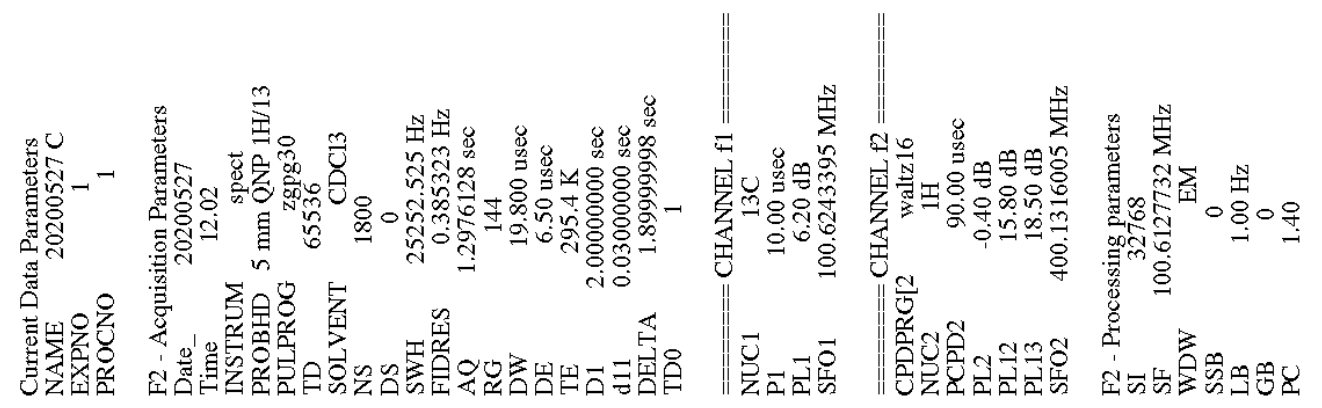

$\angle 289^{\circ} 9 L$

$0000^{\circ} \mathrm{LL}$

Z8IE $L L$<smiles>O=C(C(=O)c1ccc(C(F)(F)F)cc1)c1ccccc1</smiles>

미
0
0

stzZ:6I I

$\angle Z t 6^{\circ}$ IZI

I $\$ \$ 9^{\circ} \nabla Z I$

IZL6 SZI

zst0.9ZI

L080.9ZI I

$\neg Z 9 \varepsilon^{\circ} L Z \mathrm{I}$

ZSEI' 6 ZI

$2796^{\circ} 6 \mathrm{ZI}-$

$\checkmark L O Z$ O $\mathrm{EI}$

э0SS'ZEI

9I $\nabla Z^{\prime} \subseteq \mathcal{} \mathrm{I}-$

I $\mathcal{E} \subseteq \mathcal{\subseteq} \mathrm{I}$

399 '`E I

9 I99 $\mathcal{} \subseteq$ I

$0686^{\circ} \mathrm{SEI}$

E60E' $9 \mathcal{E}[$

$00200^{\circ} \varepsilon 6 \mathrm{I}-$

$8097^{\circ} \mathrm{E} 6 \mathrm{I} \longrightarrow$

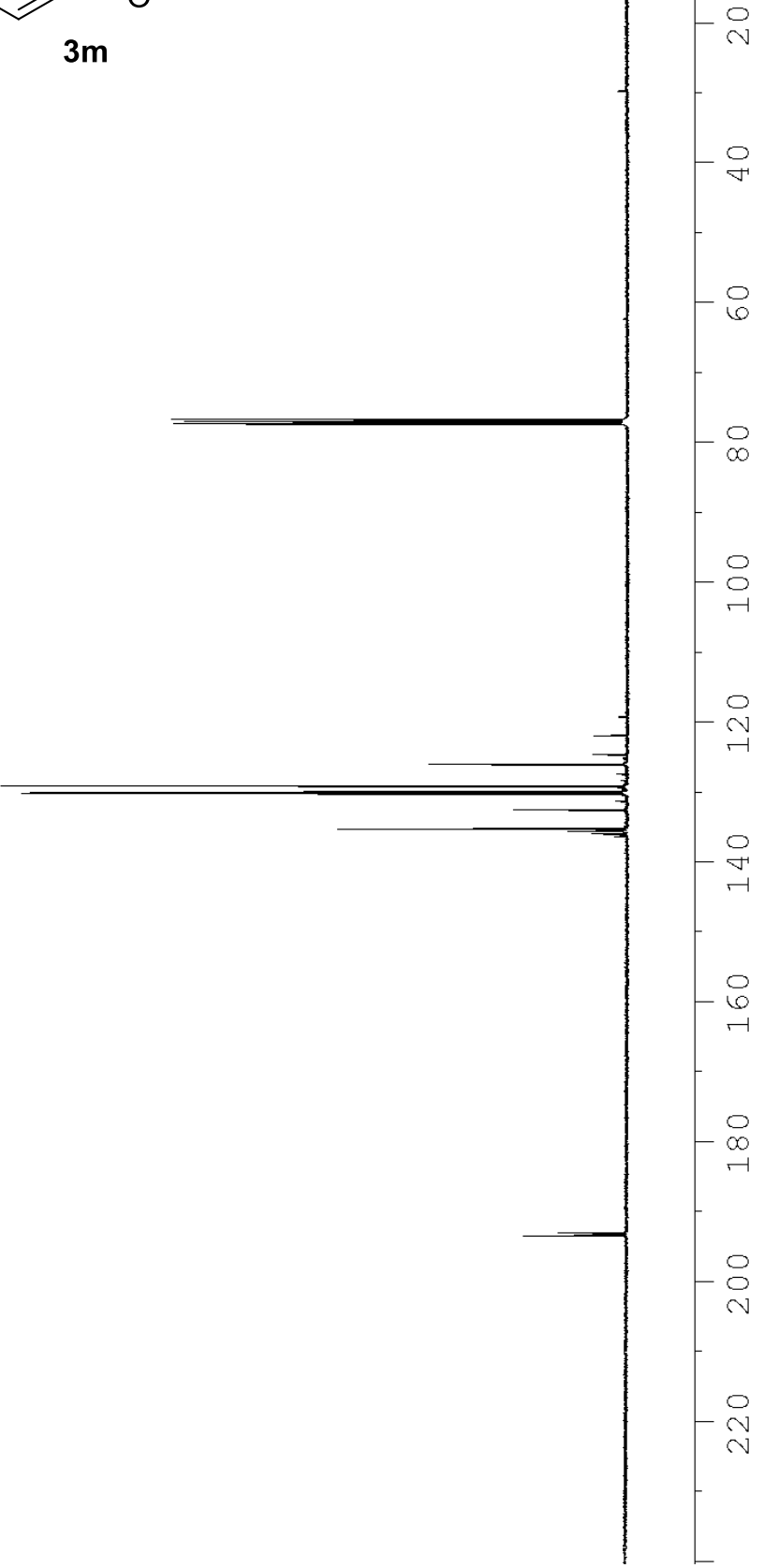




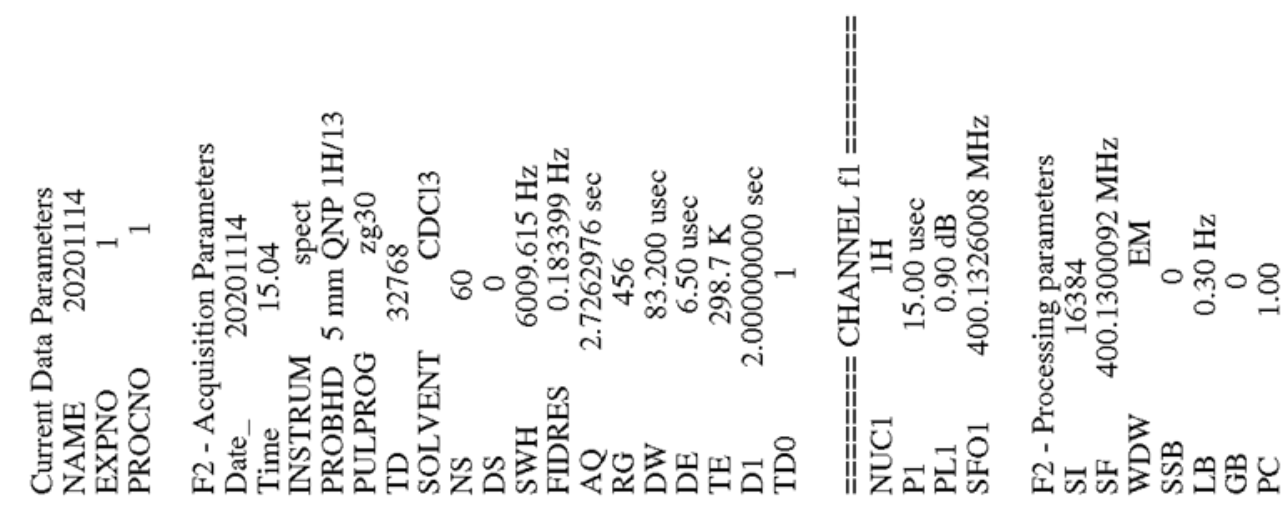

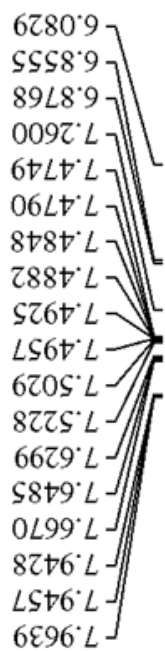<smiles>O=C(C(=O)c1ccc2c(c1)OCO2)c1ccccc1</smiles>

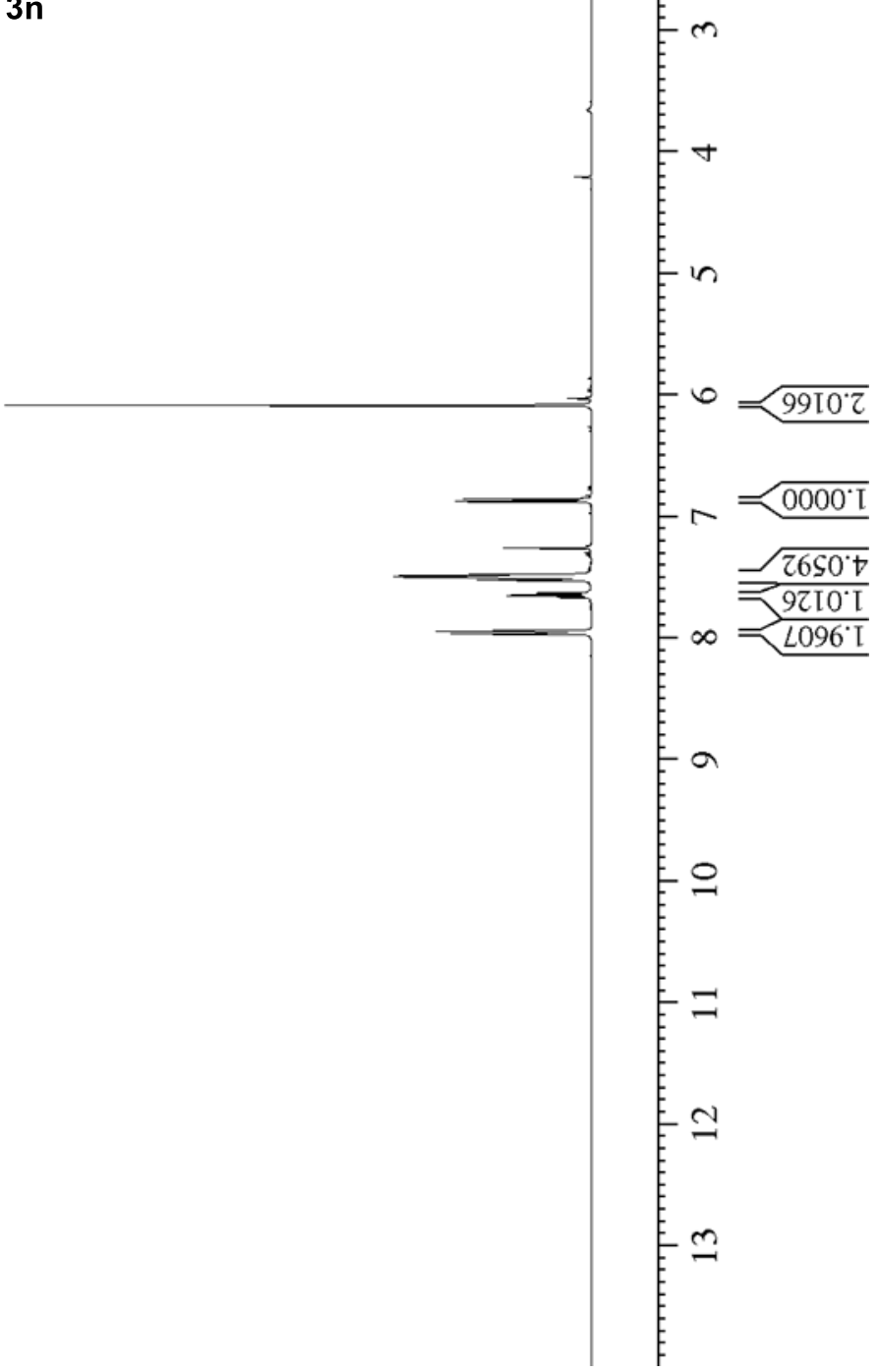




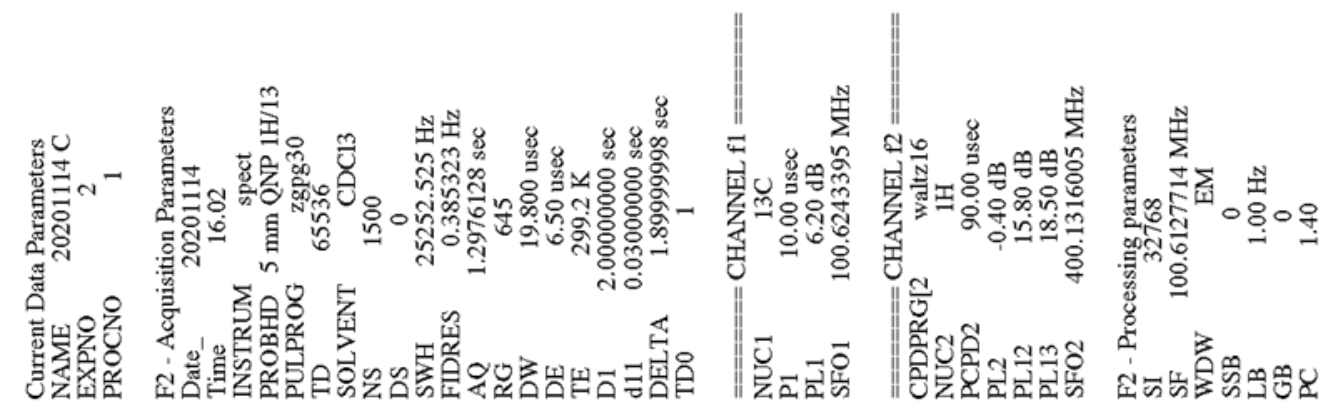

$9789^{\circ} 9 L$

$0000^{\circ} L L$

SLIE'LL

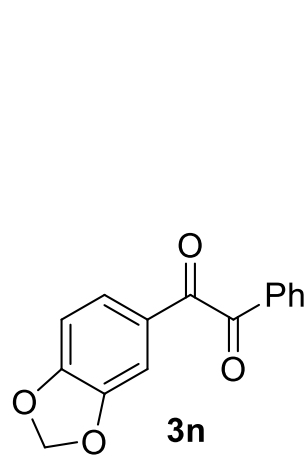

6SIZ'Z0I

S0tE'80I

$\angle \mathrm{t} 0 \mathrm{t}^{\circ} \mathrm{80I}>$

ZE98. $\angle Z \mathrm{I}$

St8 $88^{\circ} \angle Z I$

$5 S \$ 6^{\circ} 8 Z \mathrm{I}$

6I88.6ZI

Z90I ' $\varepsilon \varepsilon I$

$8 L 9 L{ }^{\circ} \mathrm{DEI}-$

9Lt9.8tI

sZLt' $\& S I$

Eع8L'Z6I

LSZS't6I -

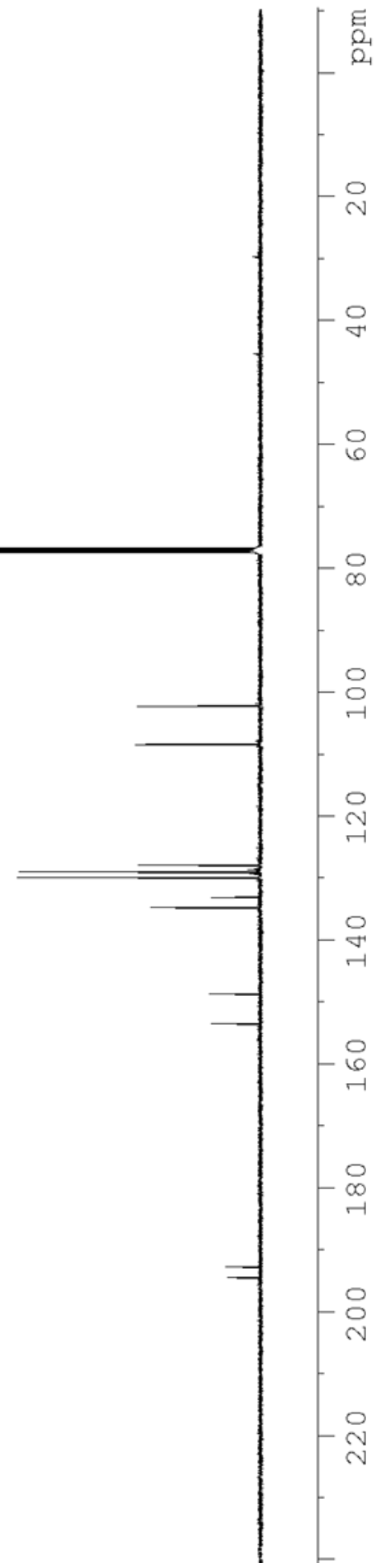




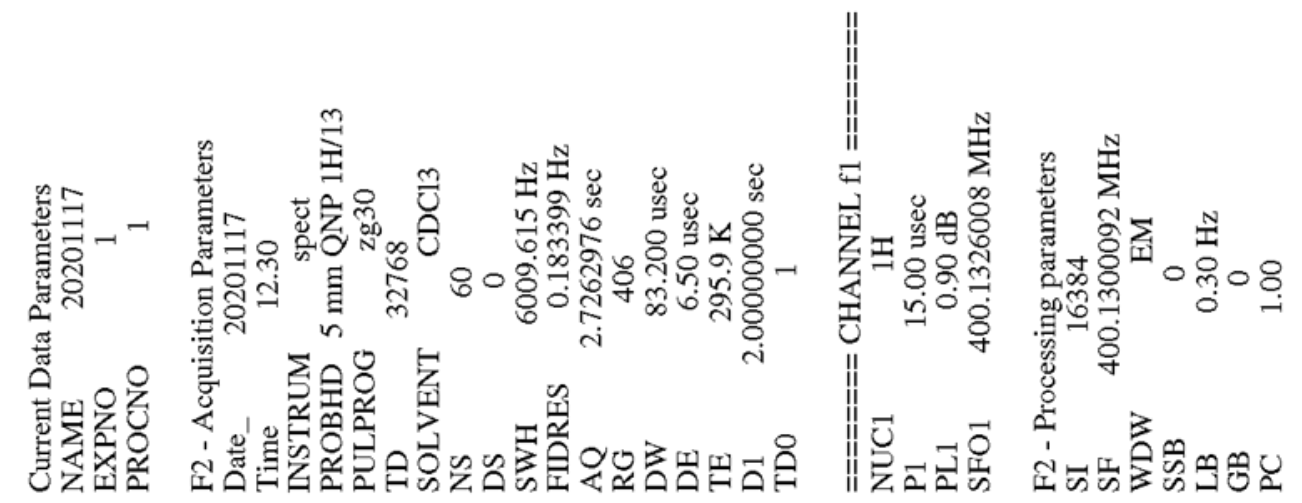

$009 \tau^{\circ} L$

OEIS $L$ -

ZZES $L$

8ISS $L$

ZLSS $L$

$86 S^{\circ} \mathrm{L}$

$86 L S^{\circ} L$

$\angle 629^{\circ} L$

$00 S 9^{\circ} L$

$\left.9259^{\circ} \mathrm{L}\right]$

$90 \angle 9^{\circ} \mathrm{L}$

$O S \angle 9^{\circ} L$

$9 \varepsilon 69^{\circ} \mathrm{L}$

$6 \mathrm{I} 68^{\circ} \mathrm{L}$

SII6 $L$

IIE6 $\mathrm{L}]$

$8\left[96^{\circ} \mathrm{L}\right]$

$\downarrow \varepsilon 86^{\circ} \mathrm{L}$

$0 \varepsilon 20.8$

$\angle S Z 00^{\circ}$

$0 t 50.8$

$9 \varepsilon 60 \%$

$\checkmark \angle 60{ }^{\circ} 8$

ZSII' 8

$68 \mathrm{II}^{\circ} 8$<smiles>O=C(C(=O)c1ccc2ccccc2c1)c1ccccc1</smiles>

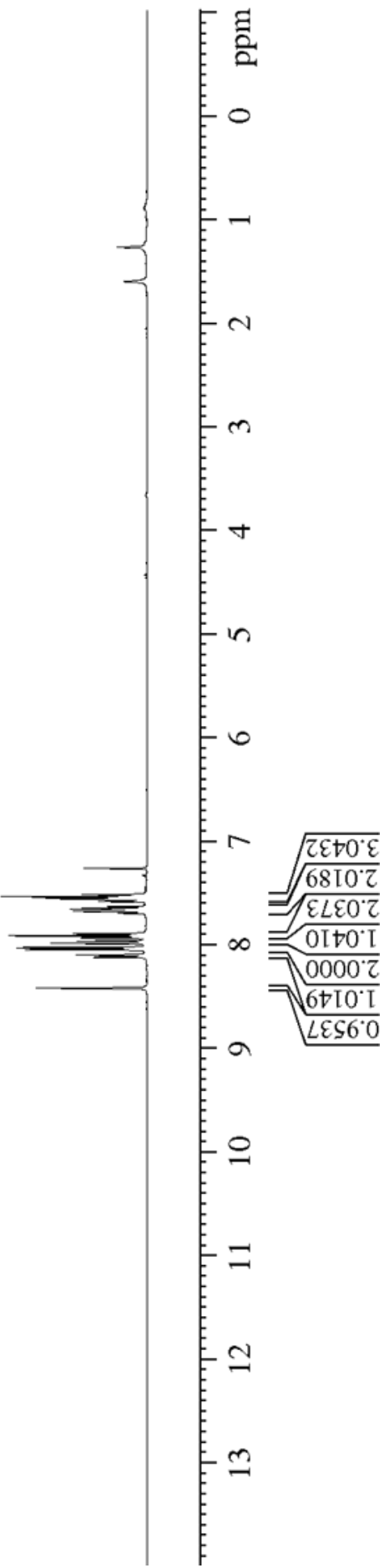




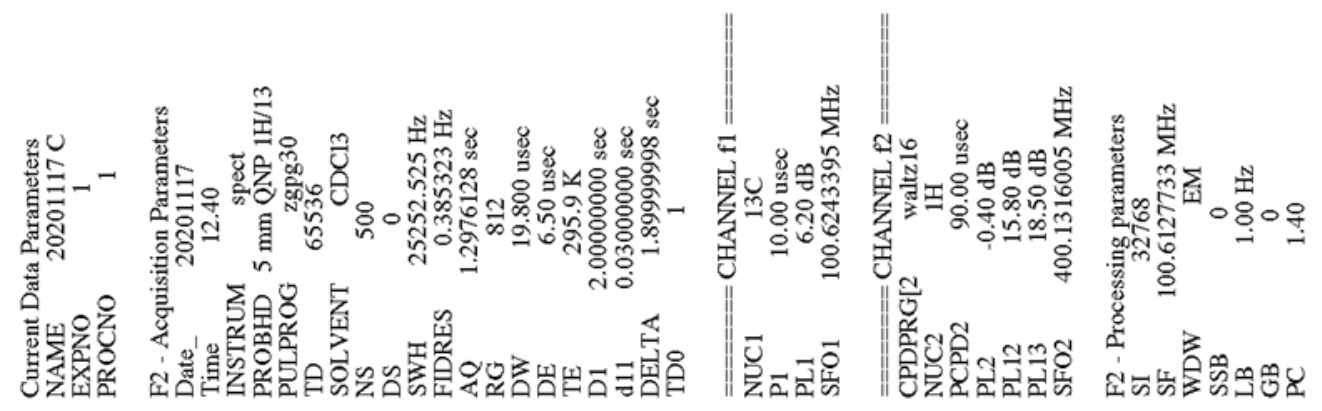

$9789^{\circ} 9 \mathrm{~L}$

$0000^{\circ} \mathrm{LL}$

6LIE'LL<smiles>O=C(C(=O)c1ccc2ccccc2c1)c1ccccc1</smiles>

30

I $\angle \varepsilon 9^{\circ} \varepsilon Z \mathrm{I}$

$769 \mathrm{I}^{\circ} \mathrm{LZI}$

$6 \varepsilon^{\circ} \angle Z I$

Zع\&0.6ZI

$089 \mathrm{I}$ '6ZI

69ع '6ZI

$\angle \mathrm{VZ6} 6 \mathrm{CI}$

2986'6ZI

ZZ6Z'0EI

88IE'حEI

$600 I^{\circ} \mathcal{E} \mathcal{E}$

$\forall L t S^{\prime} \mathcal{E} \mathcal{E I}$

$\angle 968^{\circ} \sqcup \mathcal{E} I$

[SLE'9EI]

$\varepsilon 6 \varepsilon 9^{\circ} \circ 6 \mathrm{I}$

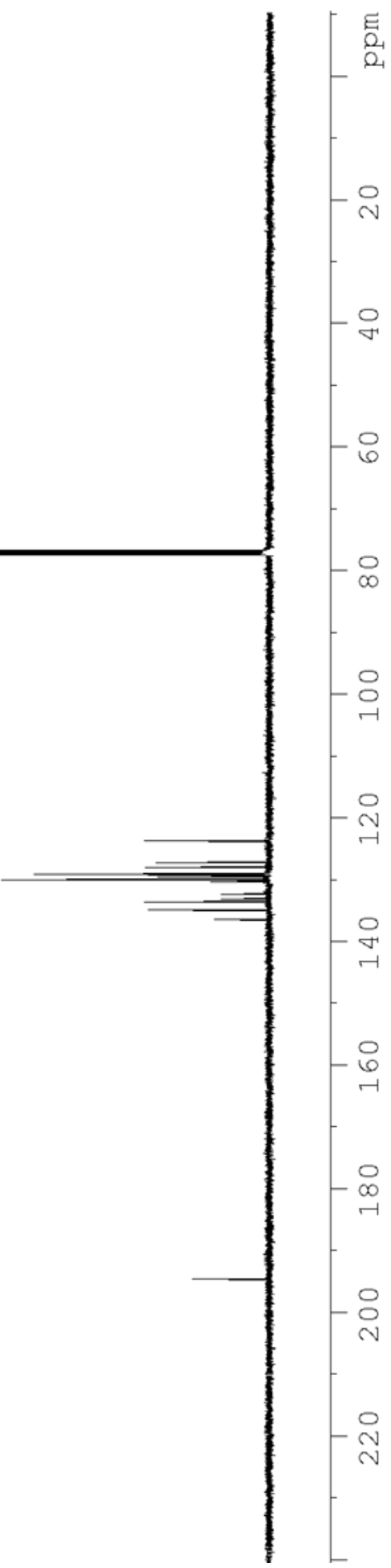



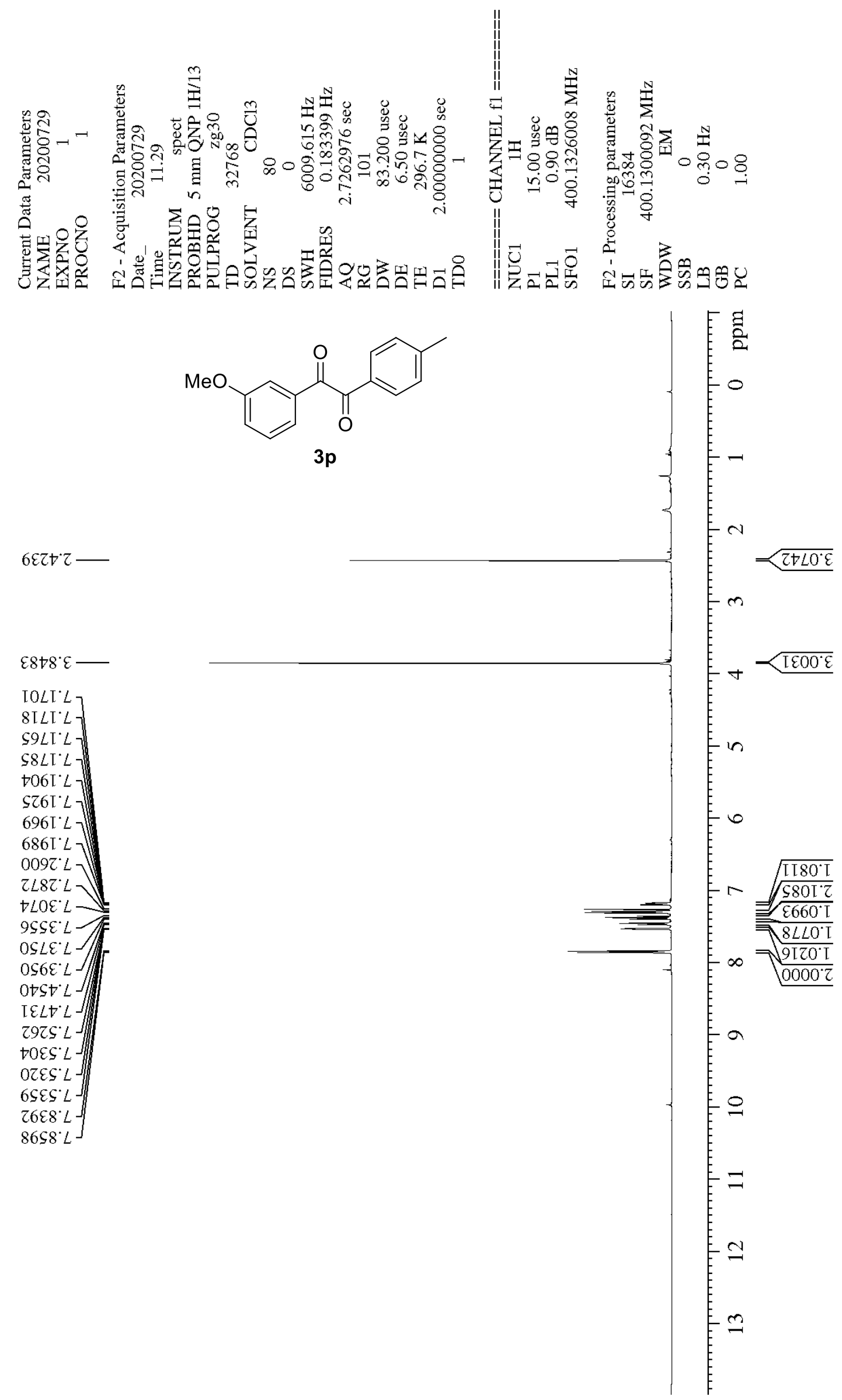

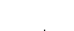




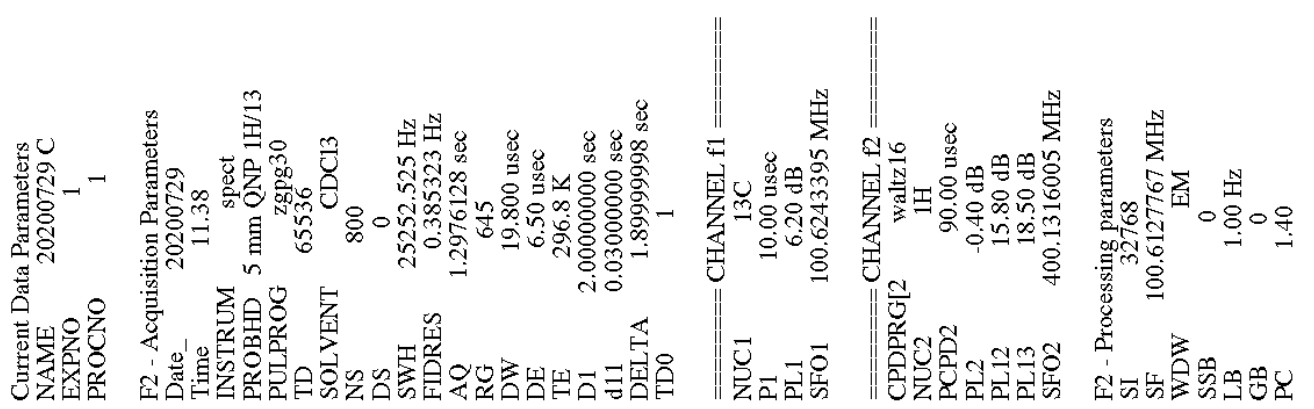

Z098.IZ<smiles>COc1cccc(C(=O)C(=O)c2ccc(C)cc2)c1</smiles>

38St'SS

$2089^{\circ} 9 L$

$\angle 866^{\circ} 9 \mathrm{~L}-$

$\varepsilon 00 Z: L$

09IE'LL-

999L'ZI L

06ZL'IZI

S9tI' $\mathrm{EZI}$

\$289.6ZI

$\$ 806.6 \mathrm{ZI}-$

9 ZOS OEI

$86 L Z^{\circ} \sqcup E I$

29LI'9tI

$6886^{\circ} 6 \mathrm{SI}$

$t 7 L I^{\circ} t 6 \mathrm{I}$
$9 L 59^{\circ}+6 \mathrm{I}$

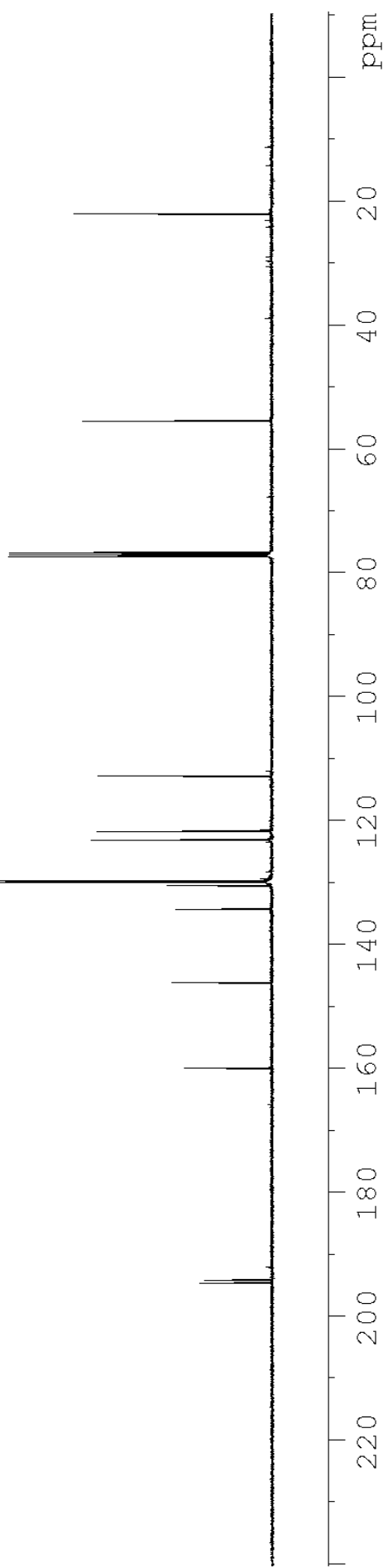




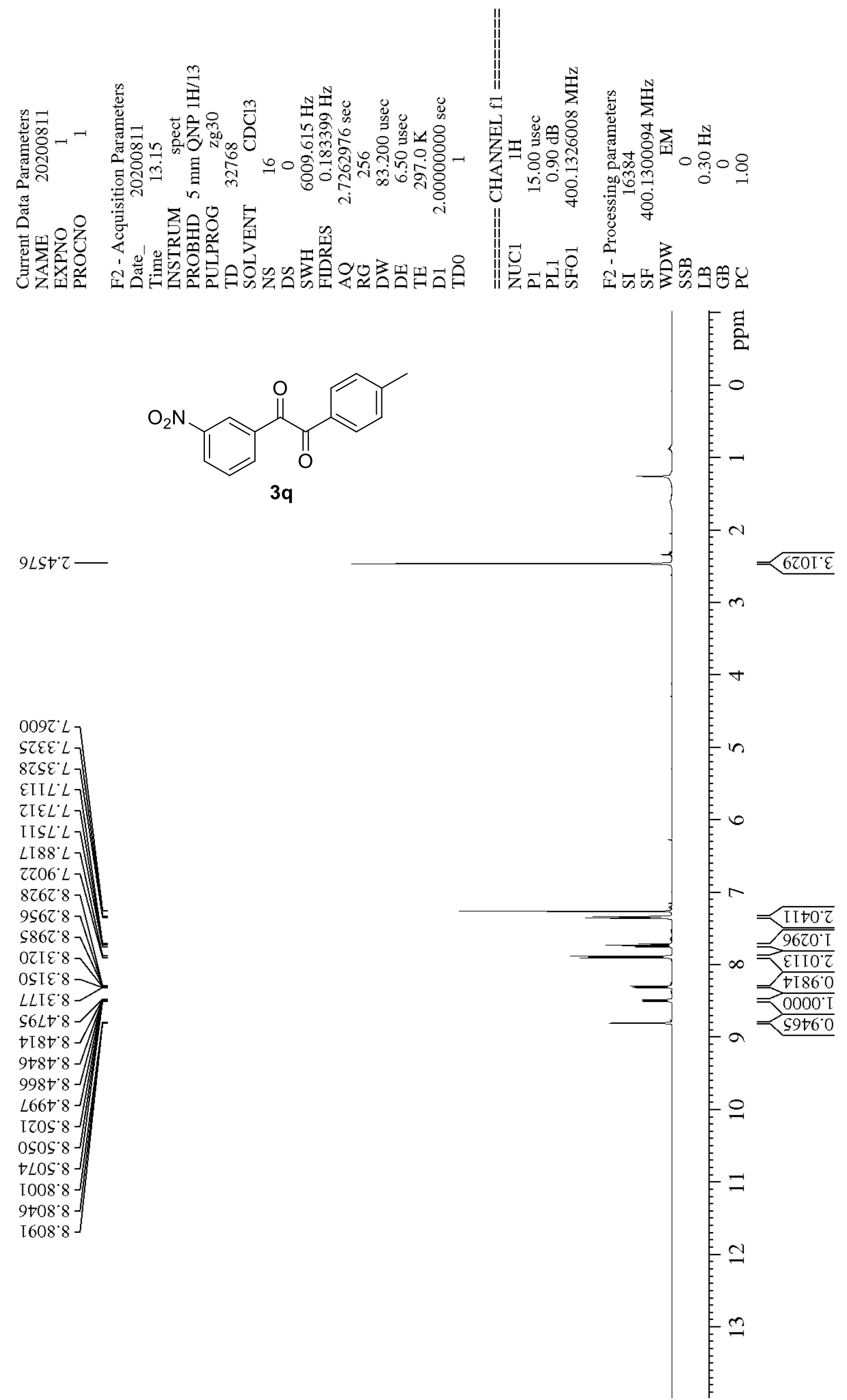




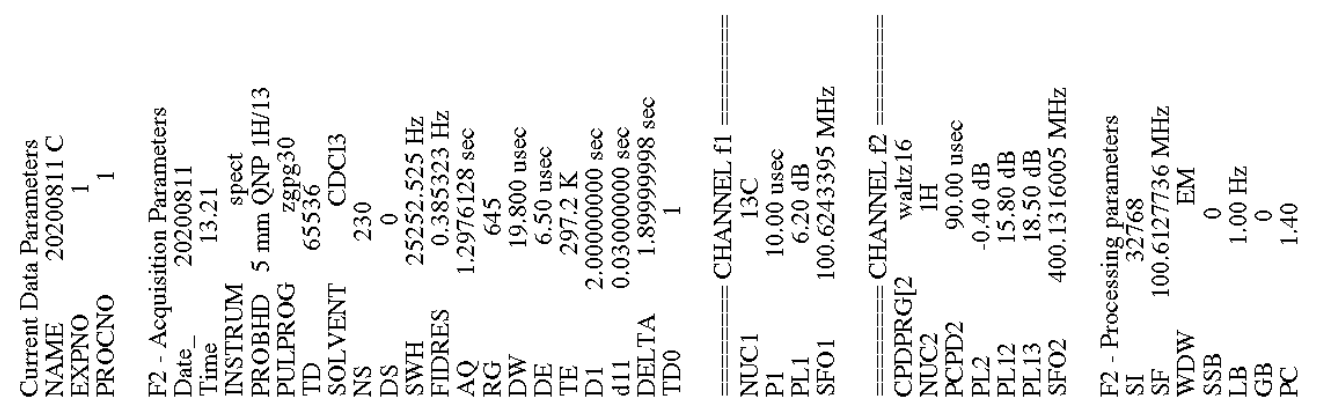

t9L6.IZ<smiles>Cc1ccc(C(=O)C(=O)c2cccc([N+](=O)[O-])c2)cc1</smiles>

$\angle 189^{\circ} 9 L$

$1000^{\circ} \mathrm{LL}$

OLIE LL

$3 q$

$\downarrow 6 \varsigma \varsigma^{\circ} \triangleright Z \mathrm{I}$

$0 \angle 69^{\circ} 8 Z \mathrm{I}$

IZZ6.6ZI

I96I 'OE I

ऽ†9Z.0E I

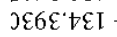

$\ni Z \subseteq Z \subseteq \mathcal{} \subseteq I$

$\angle 9 \$ 6^{\circ} 9 \mathrm{tI}$

$98 \varepsilon \varsigma^{\circ} 8 \mathrm{tI}-$

$\varepsilon 019 \cdot 16 \mathrm{I} \longrightarrow$
$8+\varepsilon \varepsilon^{\prime} \mathrm{Z} 6 \mathrm{I}$

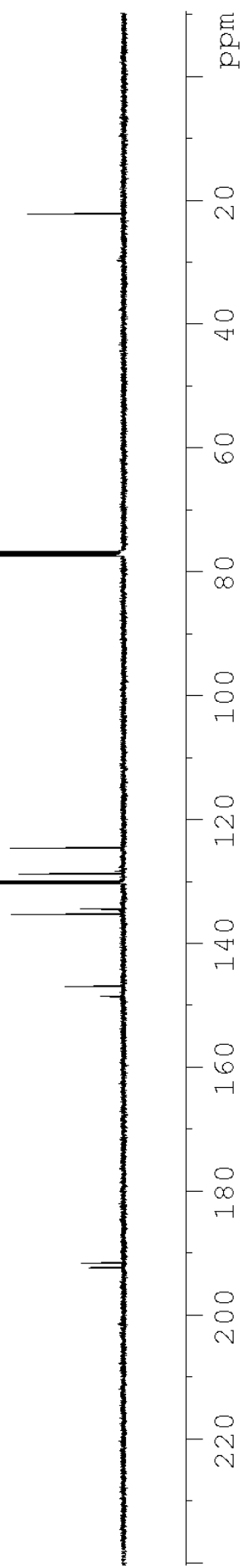




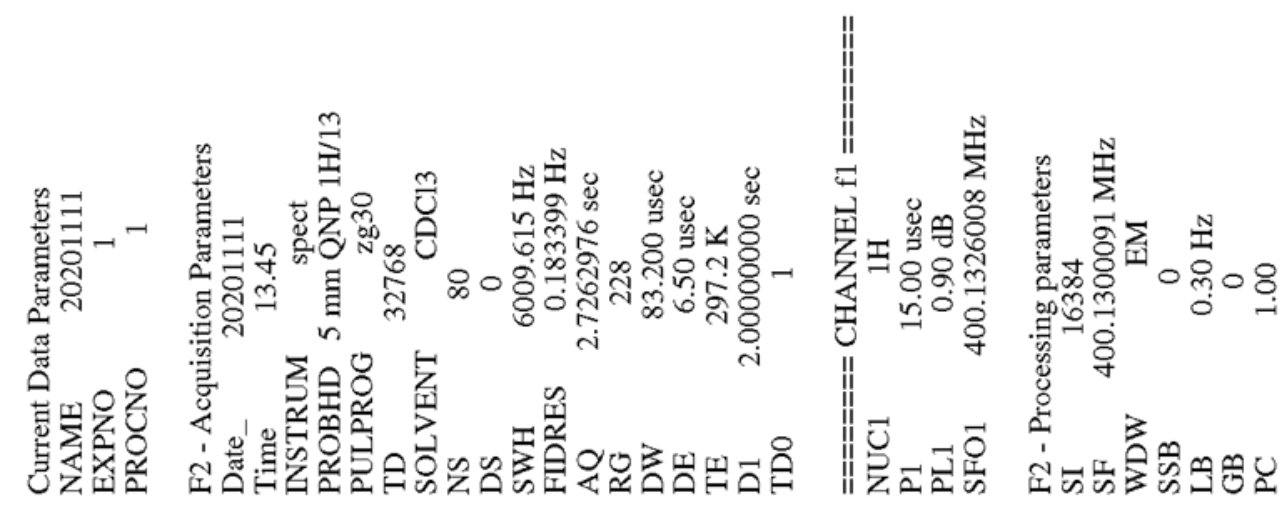<smiles>Cc1cc(C)cc(C(=O)C(=O)c2ccccc2)c1</smiles>

E09\& $\tau-$
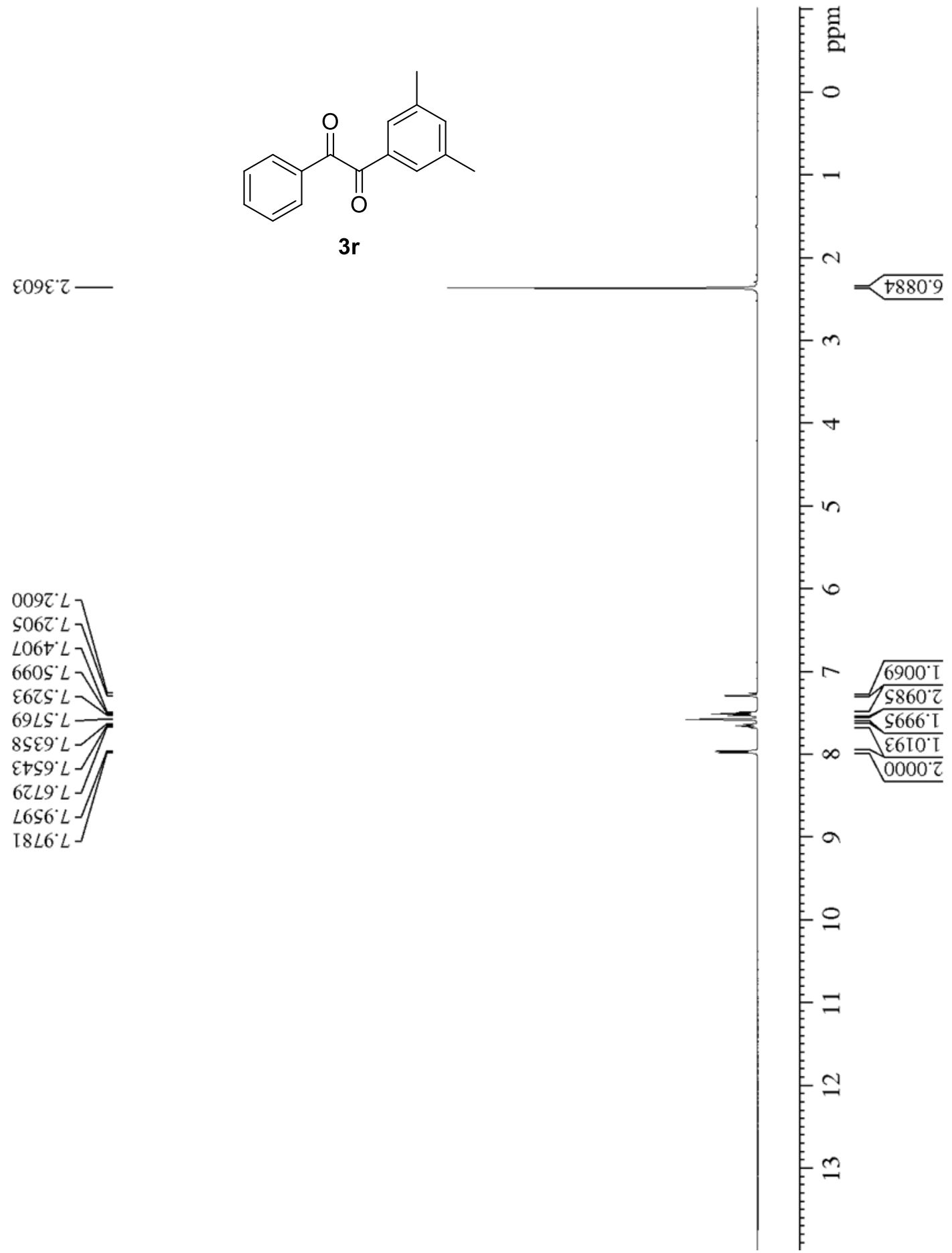


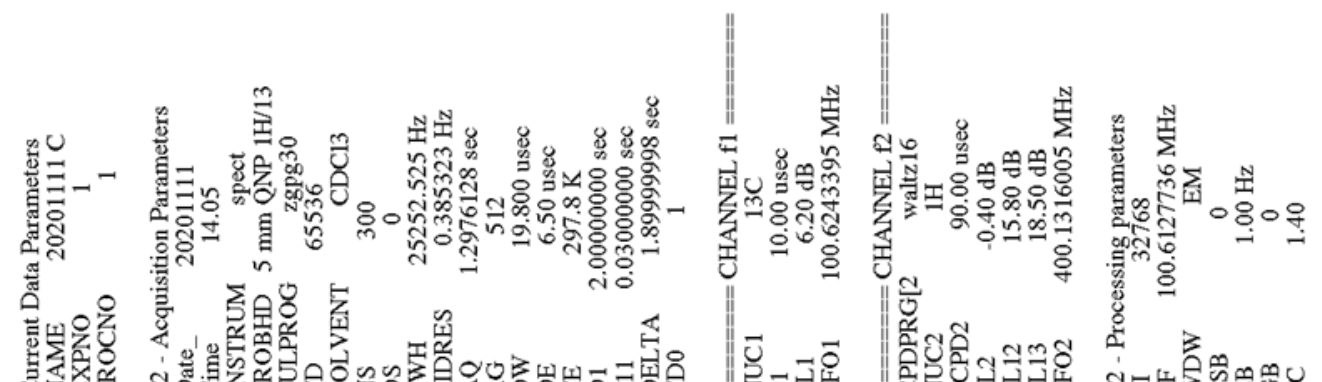

6LOI'I

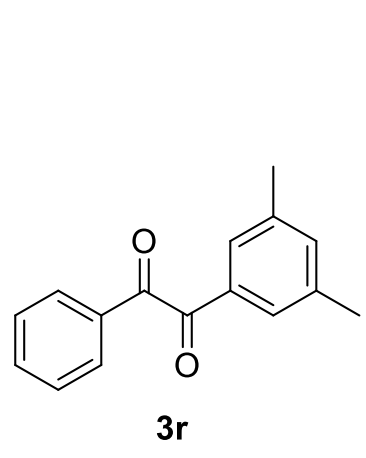

$\angle I 89^{\circ} 9 \mathrm{~L}$

O000 $L L$

9LSS'LZI

99\$6.8ZI

OSL8'6ZI

6I00 $\mathcal{E} \mathcal{\circ I}$

E6to $\mathcal{E} E \mathrm{I}$

ZI $9 L^{\circ} \downarrow \varepsilon I$

Z00L' $9 \varepsilon \mathrm{I}$

$8 \supset Z 8^{\circ} 8 \varepsilon 1$

$\varsigma 008^{\circ}+6 \mathrm{I}$

$89 \angle 0^{\circ} \mathrm{S} 6 \mathrm{I}>$

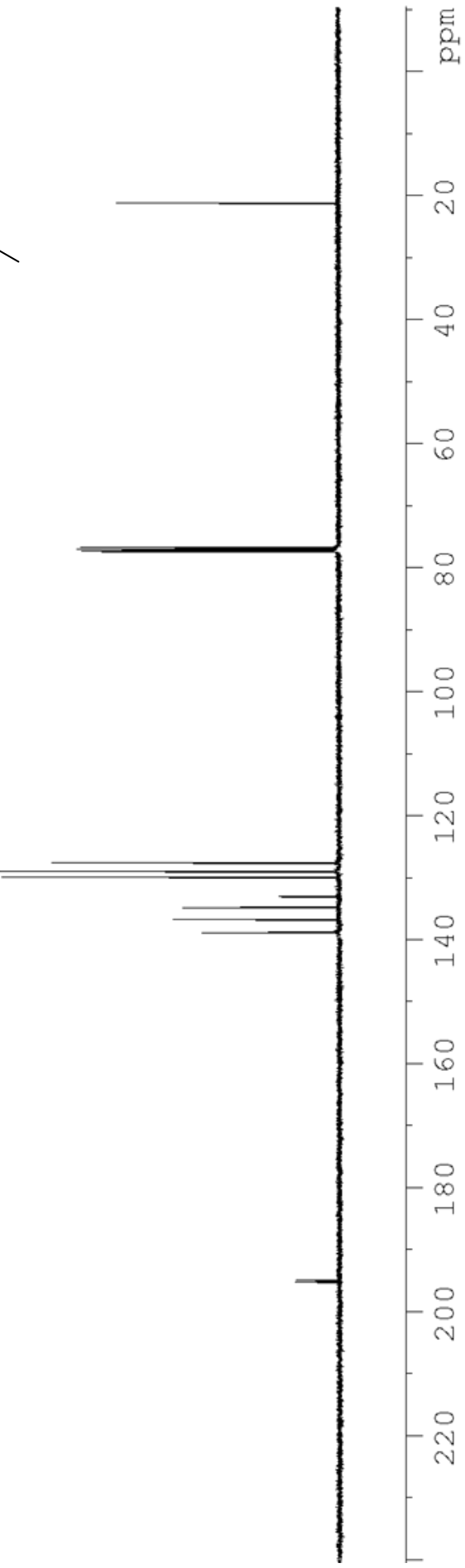




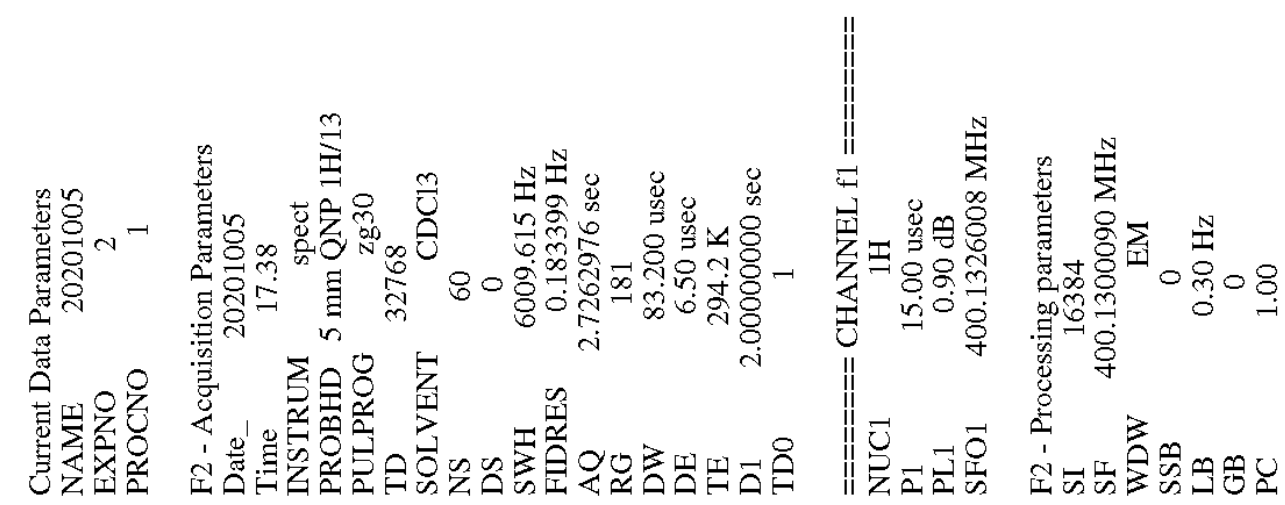<smiles>CC(=O)c1ccc(C(=O)C(=O)c2ccccc2)cc1</smiles>

3s

$\angle 9+6{ }^{\circ} \varepsilon-$

$0092: L$

$600 S^{\circ} L$

$002 S^{\circ} L$

$86 S^{2} 2$

$8959^{\circ} L$

$92 \angle 9^{\circ} L-V$

$8889^{\circ} \mathrm{L}$

ZI69 $\mathrm{L}$

ZLS6 $\angle$

†096 L

E8L6L L

$62208^{\circ}$

$0+t 0.8$

6ttI:8

$0991^{\circ} 8$

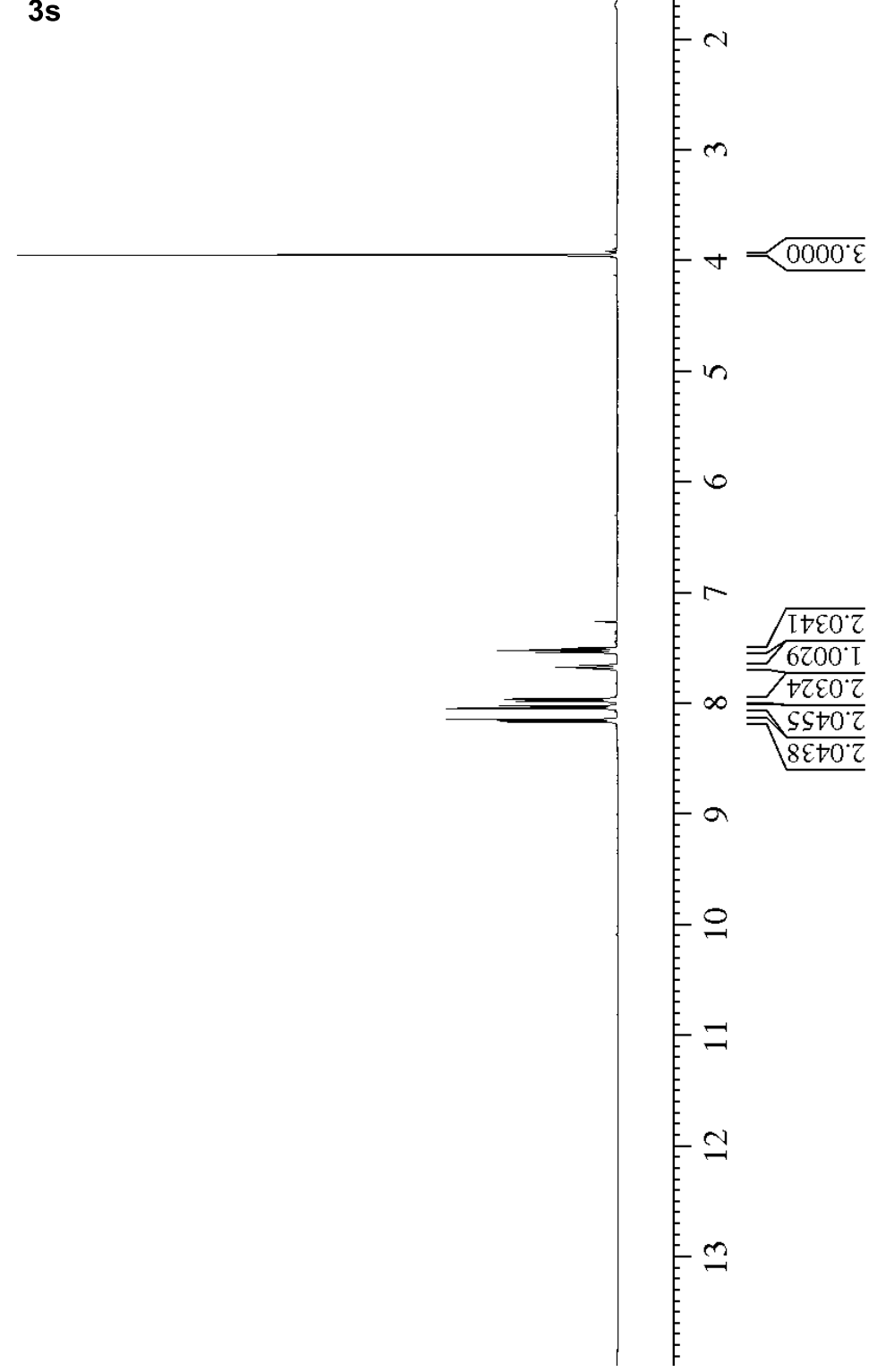




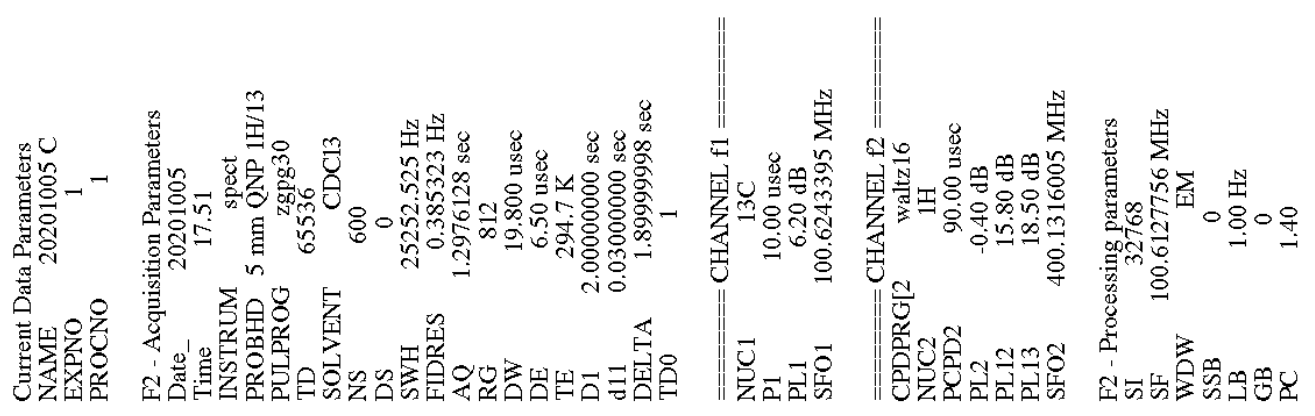<smiles>CC(=O)c1ccc(C(=O)C(=O)c2ccccc2)cc1</smiles>

3s

zE6S'zS

$\varepsilon 289^{\circ} 9 L$

$0000^{\circ} L L$

$6 L I E L L$

SLEL $6 Z I$

S†Z6.6ZI

6St0.0EI

†SI9.7\&I

SLII'SEI

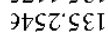

0L96 ${ }^{\circ}$ E I -

$0+28 . \varsigma 9 \mathrm{I}$

98I9. $E 6 \mathrm{I}$

$\varsigma 9+L^{\circ} \mathcal{E} 6 \mathrm{I} \longrightarrow$

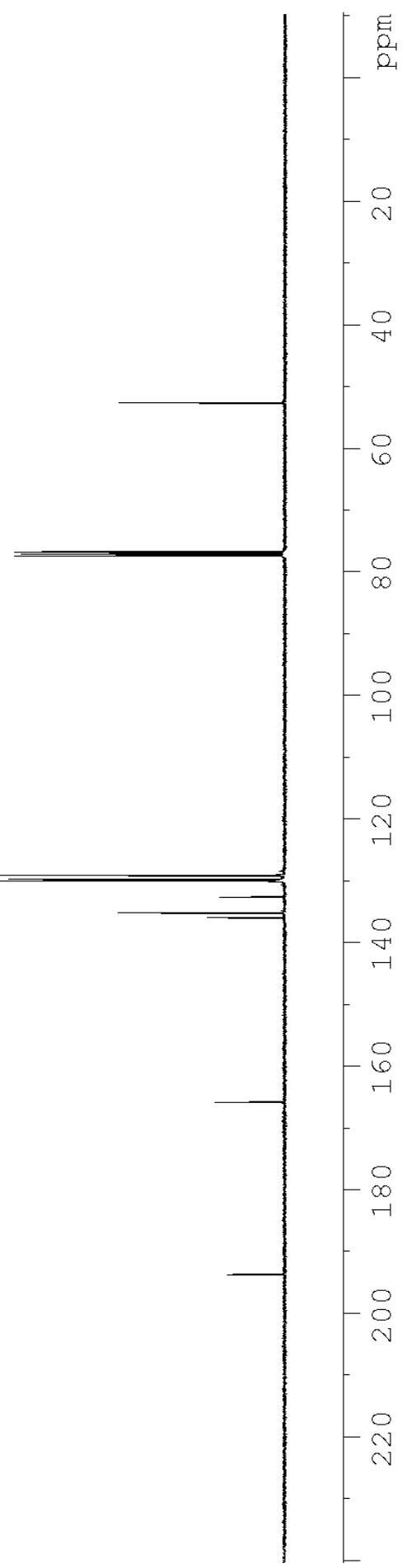



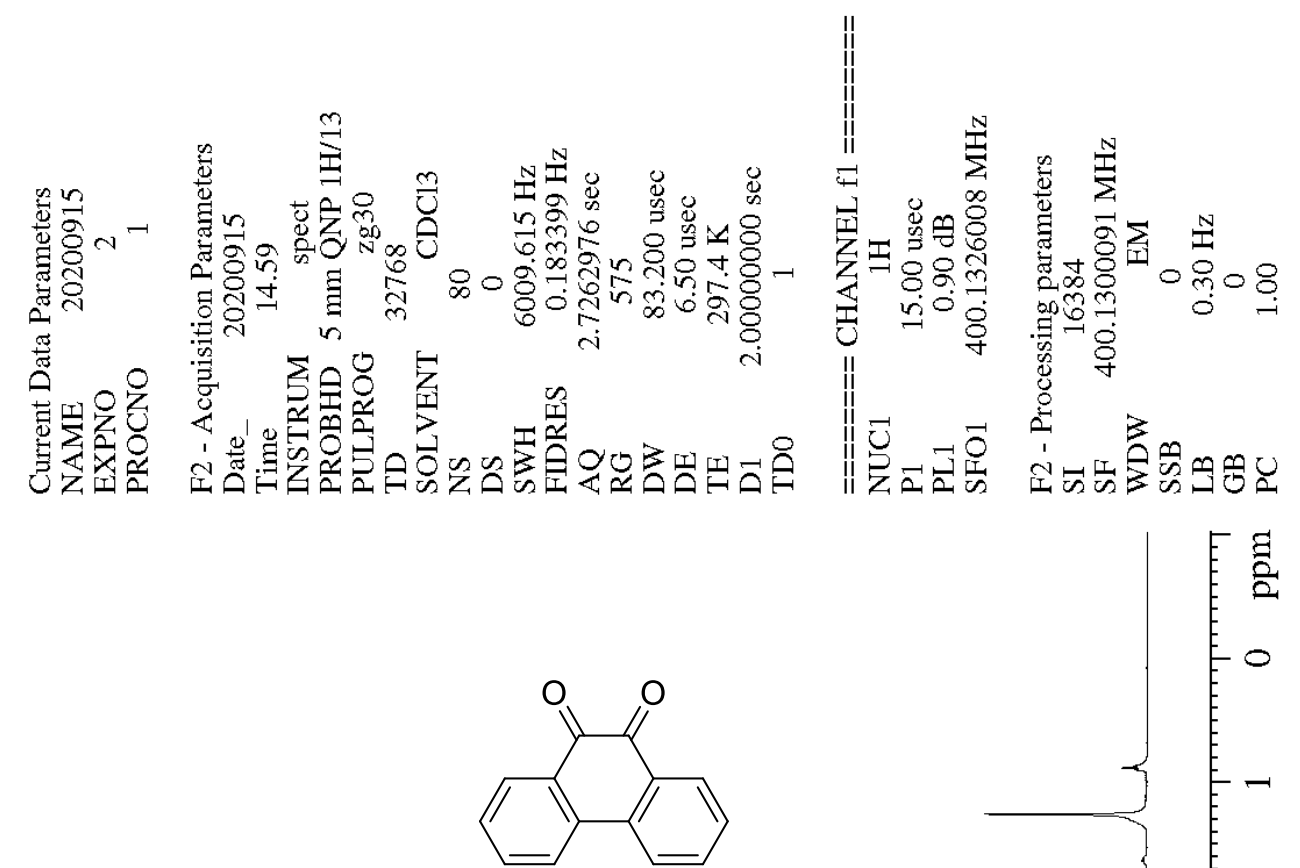

$3 t$

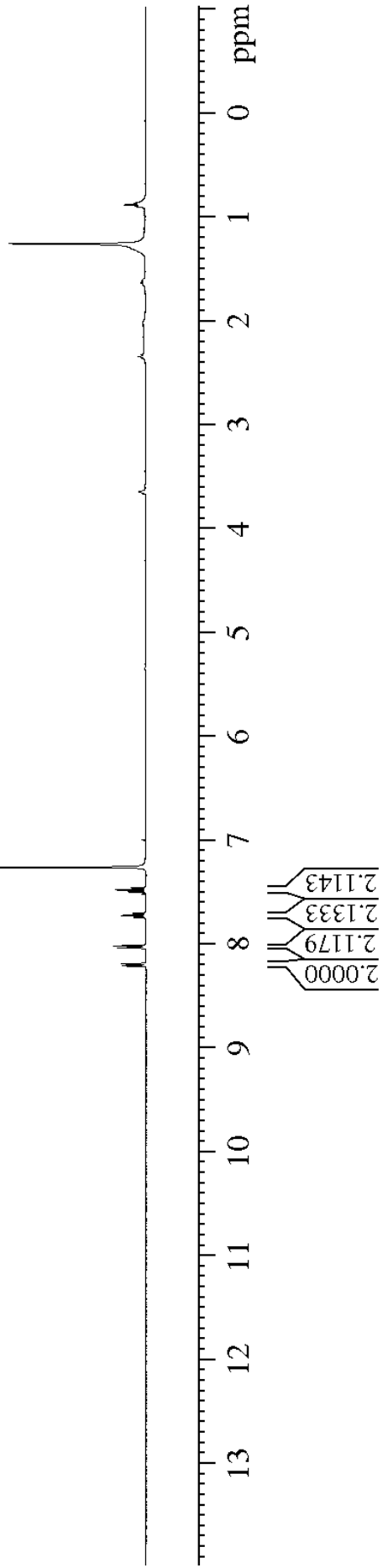

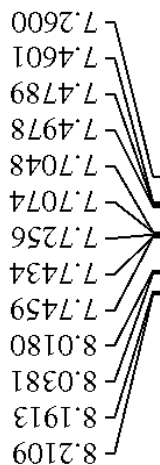



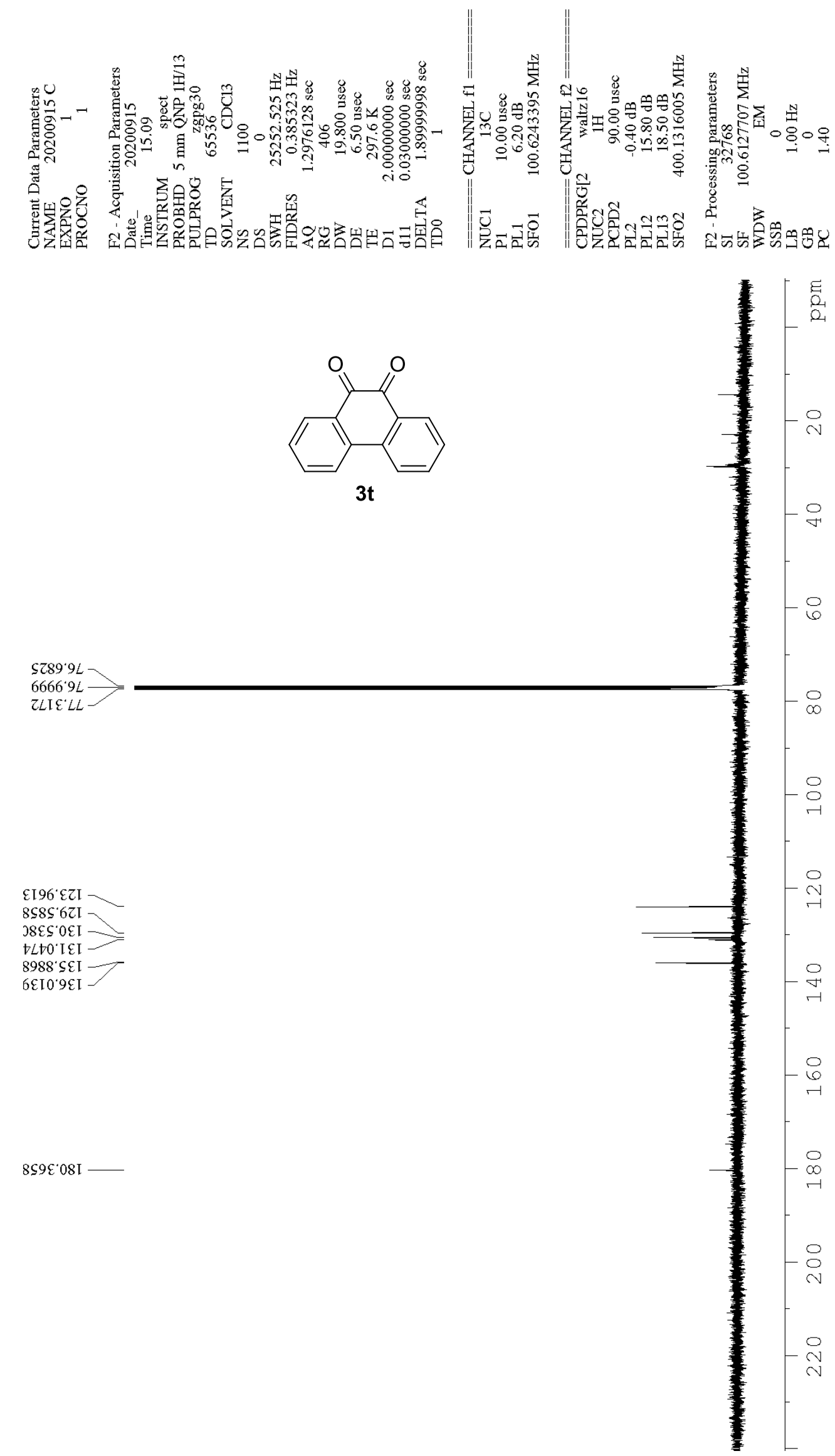

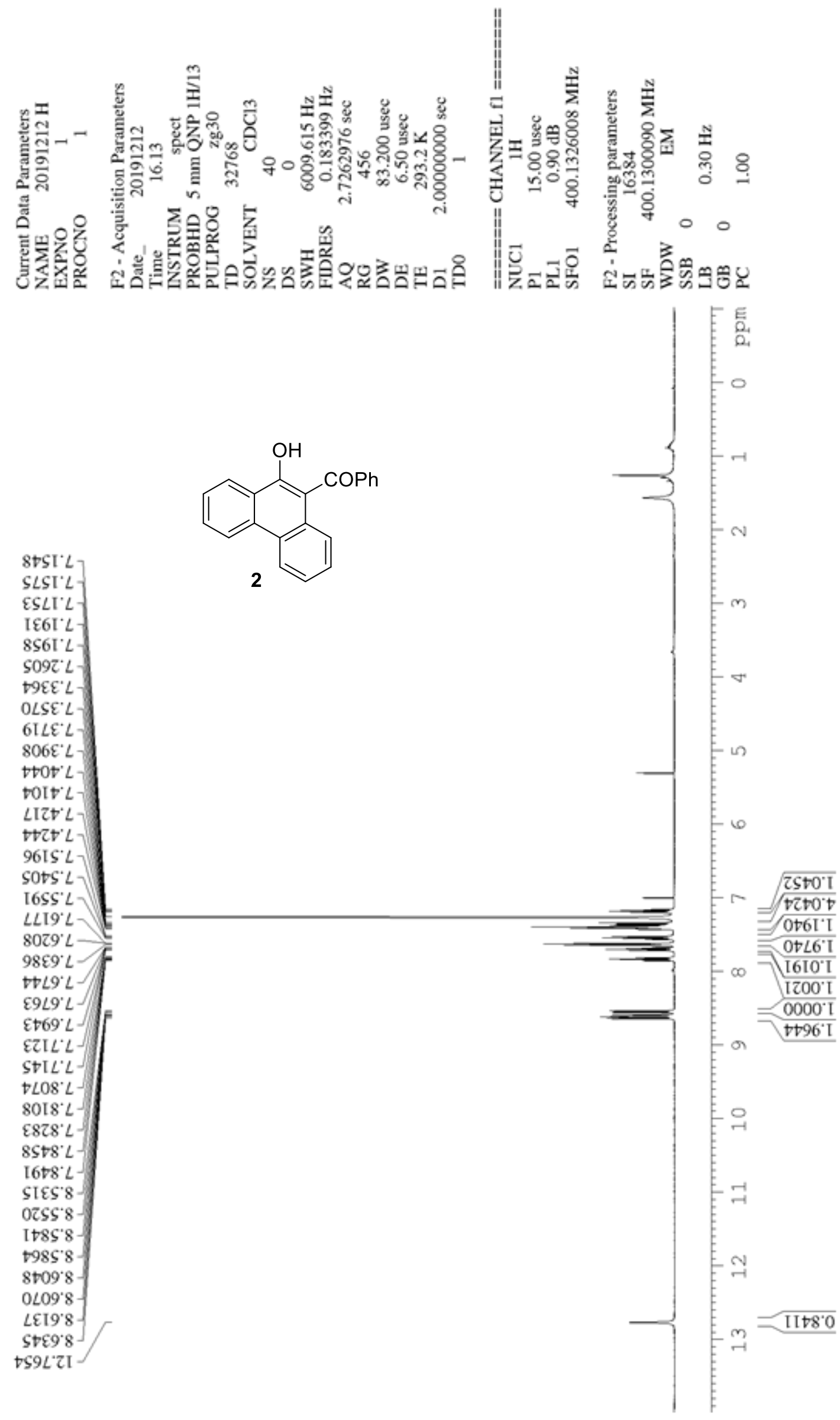

SLSI'L

ESLI'L

IE6I $L$

$8 S 6 \mathrm{I}^{\circ} \mathrm{L}$.

S09R $L$



$O L S E^{\prime} L$

6I $\angle E: L$

$806 \varepsilon^{\circ} L$

ttOt' $L$

toI $V^{*} L$

$t \nabla 2 t^{\prime} L$

96IS $L$

SOtS L $L$

I6SS' $L$

LLI9' $L$

$8029^{\circ}$

$98 \varepsilon 9^{\circ} \mathrm{L}$

$\nabla \angle \angle 9^{\circ} \mathrm{L}$

$\varepsilon+69^{\circ} \mathrm{L}$

$\varepsilon Z I L \cdot L$

$S t I L^{\prime} L$

$\checkmark \angle 08^{\circ} L$

$8018^{\circ} \mathrm{L}$ -

E8Z $8^{\circ} \mathrm{L}$
$8 \mathrm{~S}^{\circ} \mathrm{L}$

I $6788^{\circ} \mathrm{L}$

\IES'8

OzSS 8

It8 $S^{\prime} 8$

$598 \mathrm{~S}^{\prime} 8$

$8+09^{\circ} 8$

$0 \angle 09^{\prime} 8$

LEI9' 8

S๐ $89^{\circ} 8$

$\downarrow S 9 L ' Z I$ 


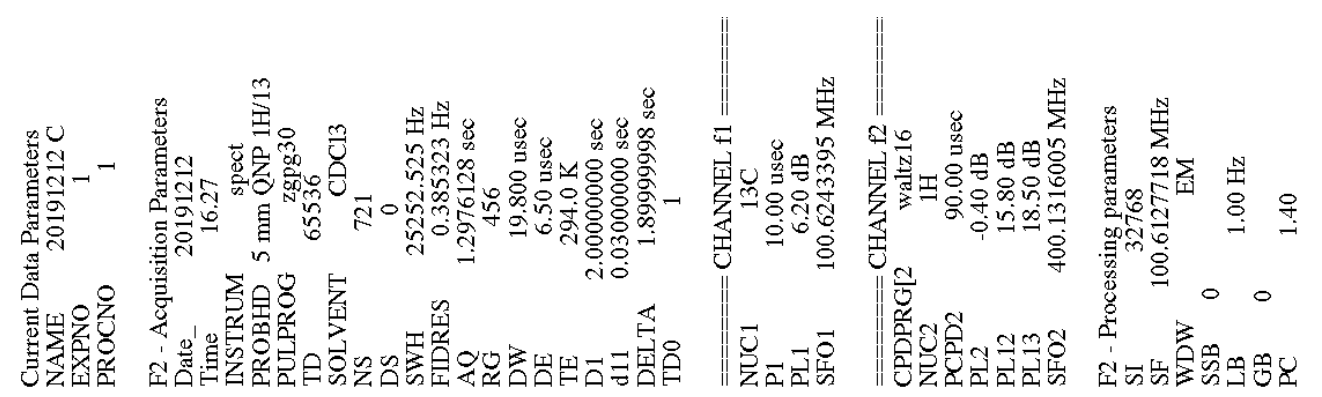<smiles>O=C(c1ccccc1)c1c(O)c2ccccc2c2ccccc12</smiles>

$0 \$ 89^{\circ} 9 L-$

$0 \varepsilon 00^{\circ} \mathrm{LL}$

$8 \subseteq 0 Z L L$

$00 Z E L L-$

\$8Z6.0I I

$\mathcal{E} 009^{\circ} \mathrm{ZZI}$

†6\&8 $\mathrm{ZZI}$

It It $t$ ZI

t6S0'SZI

t68I' $\mathrm{CI}$

$\angle E L 8^{\circ} \subseteq Z I-$

E0tI'9ZI

$\varepsilon L O I^{\prime} L Z I$

ZI $\angle 9^{\circ} \angle Z \mathrm{I}$

969 $8 \mathrm{ZI}$

09Z7 6ZI

8ZEZ $0 \varepsilon$ I

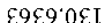

9Zt七 $Z \varepsilon \mathrm{I}$

¿E66 $\mathcal{} \mathcal{E} \mathrm{I}$

E8IE 0 t I

9\$89.09I -

$8 I \varepsilon Z 00 Z$

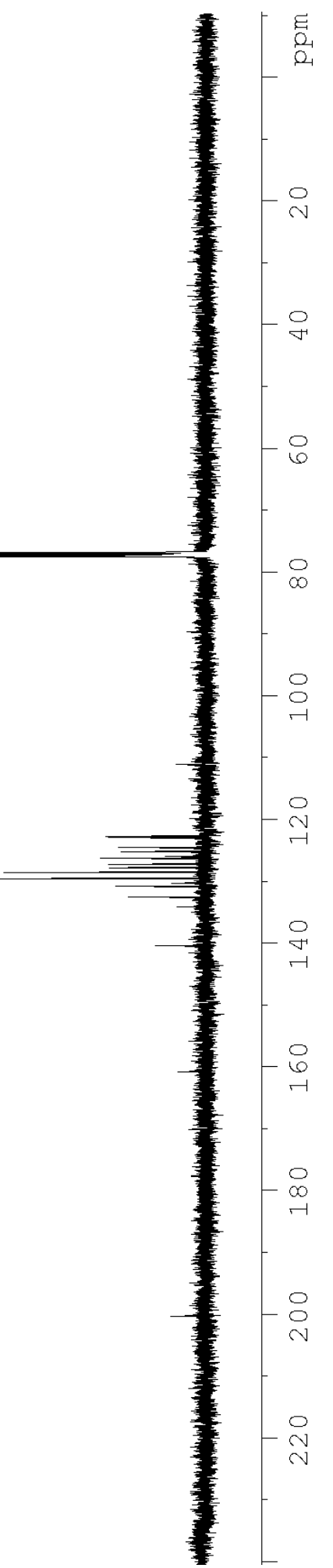




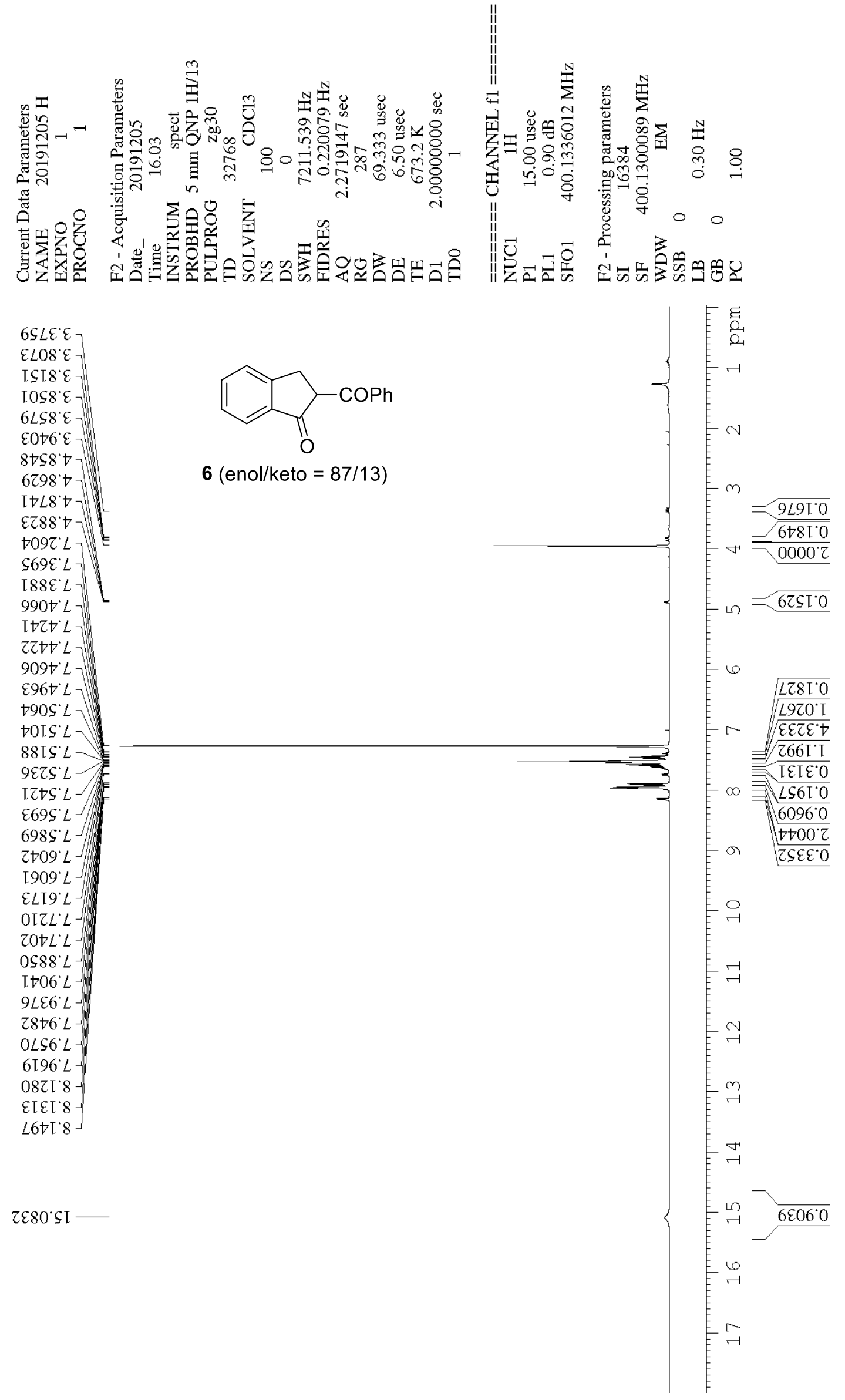



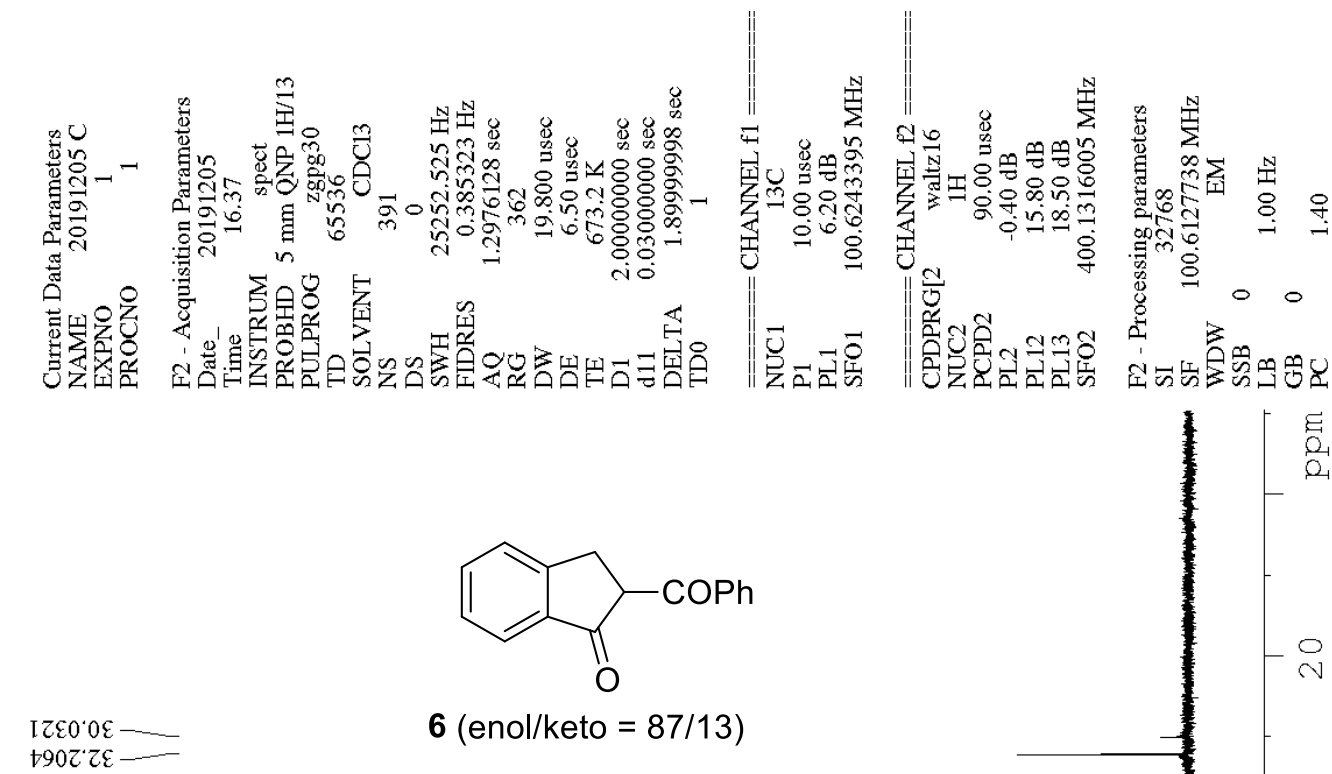

3525.95

$\subseteq \varepsilon 00^{\circ} L L$

$\$ 90 Z^{\prime} L L$

OIZE $L L$

S8Zt $60 \mathrm{I}$

I0Et $\mathcal{E} \mathrm{I}$

$9729^{\circ} \circ Z \mathrm{I}$

St0S'9ZI

$t S t T \cdot L Z I$

$\$ Z 89^{\circ} \mathrm{LZI}$

Z860.8ZI

$9 \varepsilon 6 \mathrm{~S}^{\circ} 8 \mathrm{ZI}$

$86 \mathrm{I8} .6 \mathrm{ZI}-$

бZSZ'IEI

วZE'EEI -

tLES'EEI

бSLL' $\downarrow \mathcal{E} \mathrm{I}$

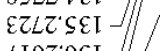

LI9Z'9EI J

LLL8 $L E[-$

StSS' $8 \mathrm{tI}$

ZOEL $L^{\circ} 0 L \mathrm{I}$

$\mathrm{I} \angle \$ 8^{\circ} \mathrm{\varsigma} 6 \mathrm{I}-$

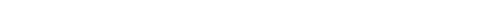

ह1
01
01 

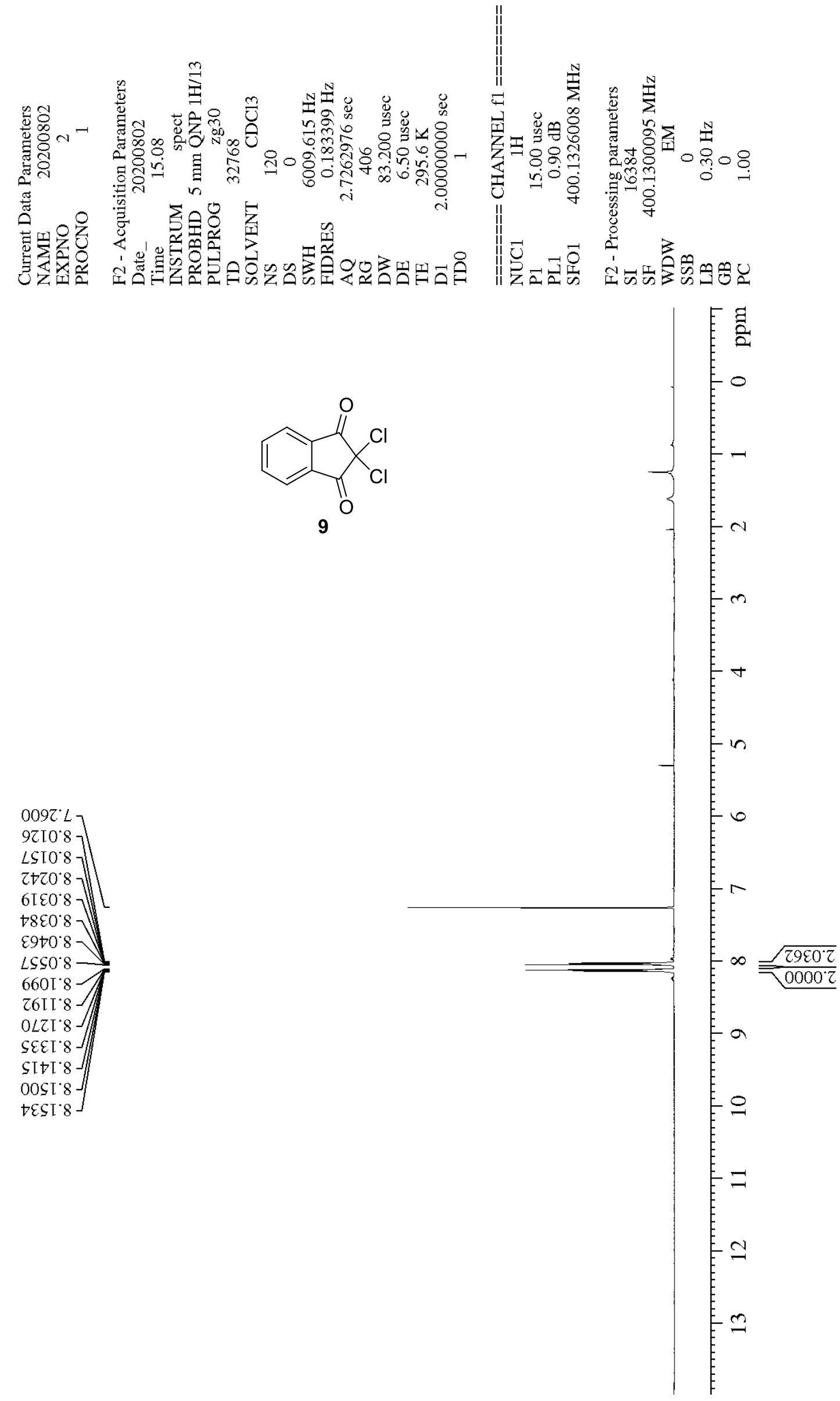


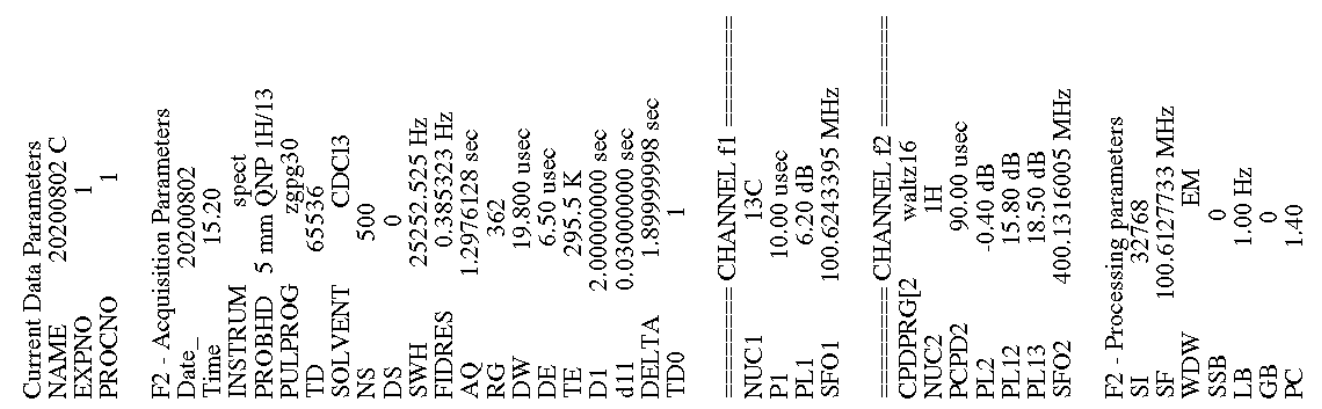

$069 L ' Z L$

$\angle 289^{\circ} 9 L$

$\mathrm{I} 000^{\circ} \mathrm{LL}$

$\mathcal{E Q O Z} L L$

I8I $\mathcal{E} L L$

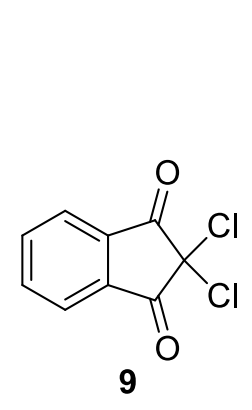

LZSL'SZI

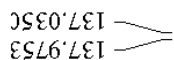

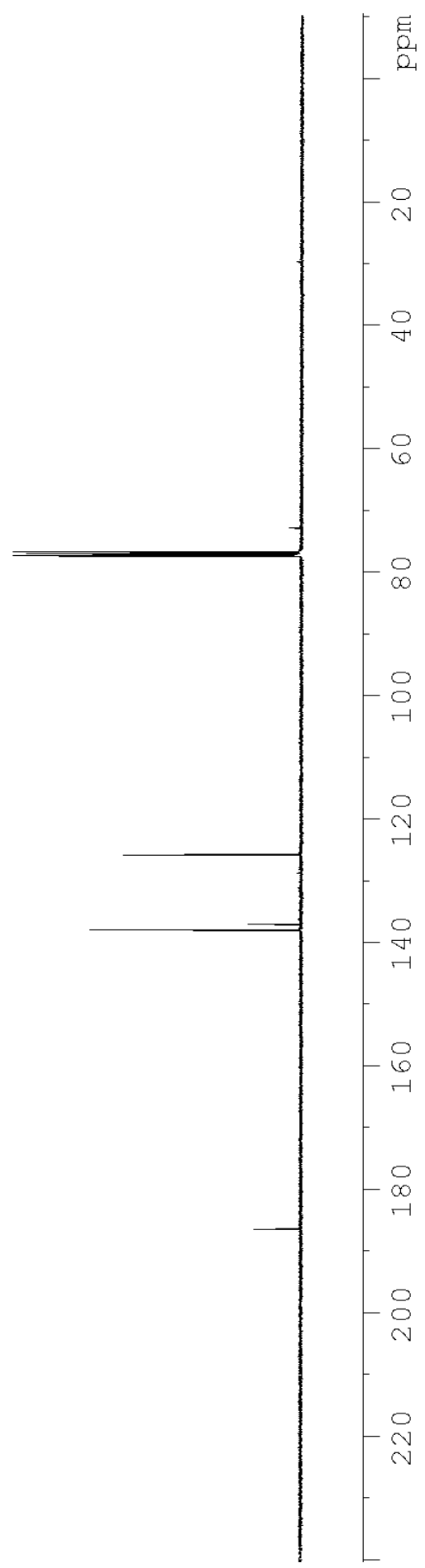

S097.98I 
5) ${ }^{1} \mathrm{H}$ and ${ }^{13} \mathrm{C}-\mathrm{NMR}$ spectra of ${ }^{13} \mathrm{C}-5 \mathrm{~b},{ }^{13} \mathrm{C}-1 \mathrm{~b}$ and ${ }^{13} \mathrm{C}-3 \mathrm{~b}$
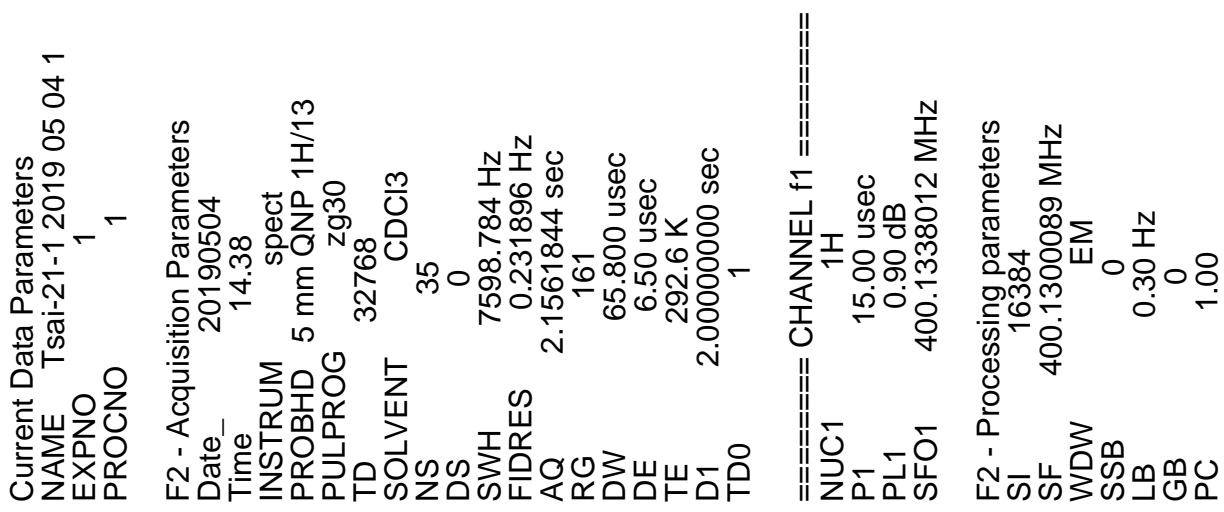

86レナ゙レ

Gเ08'大

$7999: 9$

$\angle \nabla \angle 0^{\circ} \angle$

$6692^{\circ} L$

$0087^{\circ} L$

$\angle L 6 t^{\circ} \angle$

ILIG' $\angle$

$0 \angle \nabla S^{\circ} \angle$

OS9G' $\angle$

$1686^{\circ} \mathrm{L}$

$2266^{\circ} \mathrm{L}$

ट०10.8

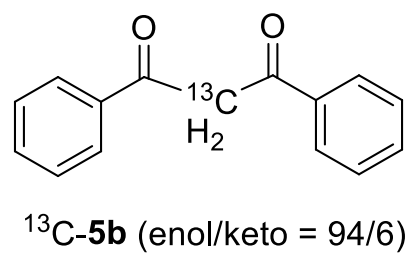

${ }^{13} \mathrm{C}-5 \mathrm{~b}($ enol $/$ keto $=94 / 6)$

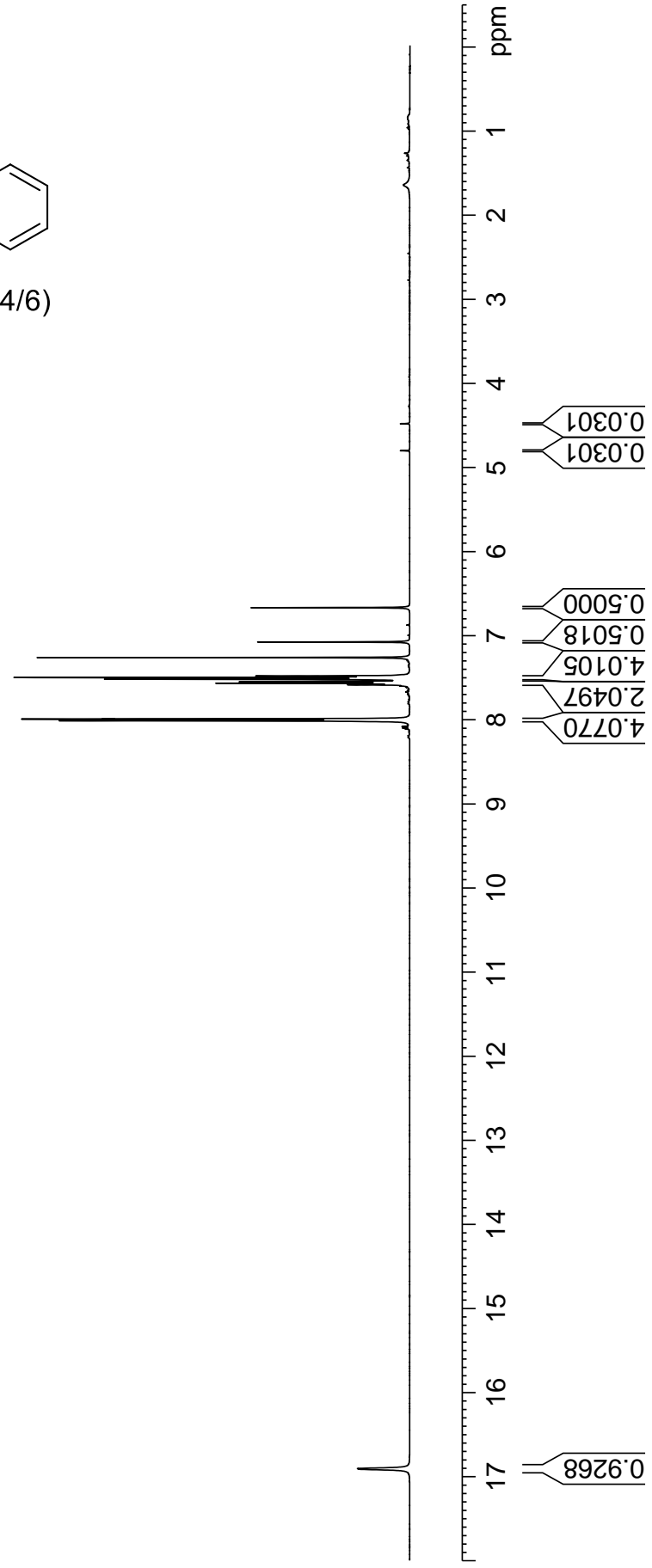



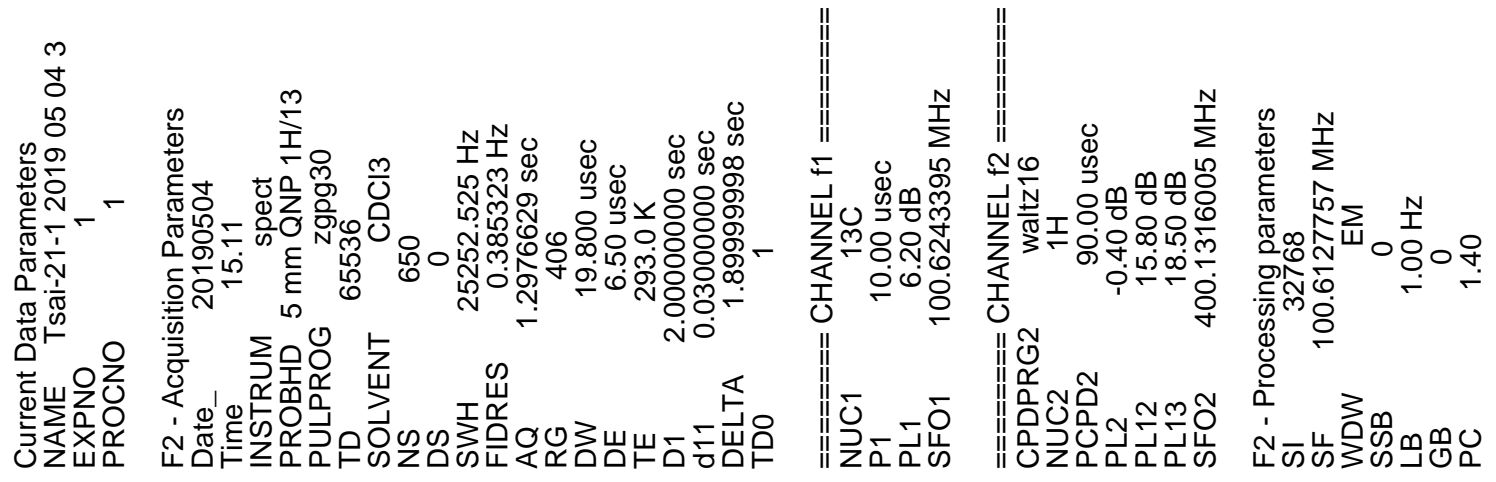

เ乙Lで0૬<smiles>O=C([13CH2]C(=O)c1ccccc1)c1ccccc1</smiles>

${ }^{13} \mathrm{C}-5 b($ enol $/$ keto $=94 / 6)$

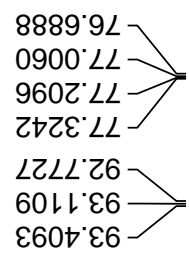

Z9Zト LZト

G己tレ'L己L

E699.8ट เ

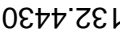

LOE† $9 \varepsilon$

เトLCSEL

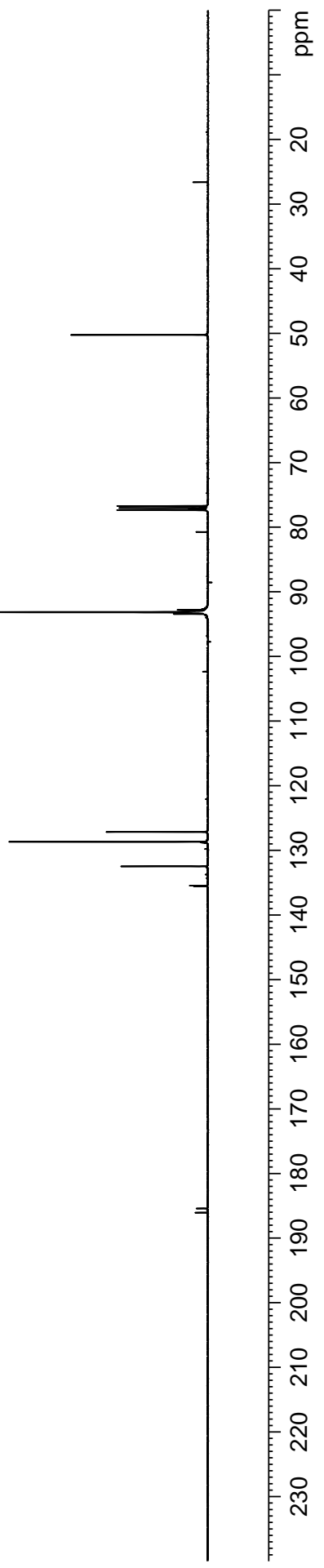




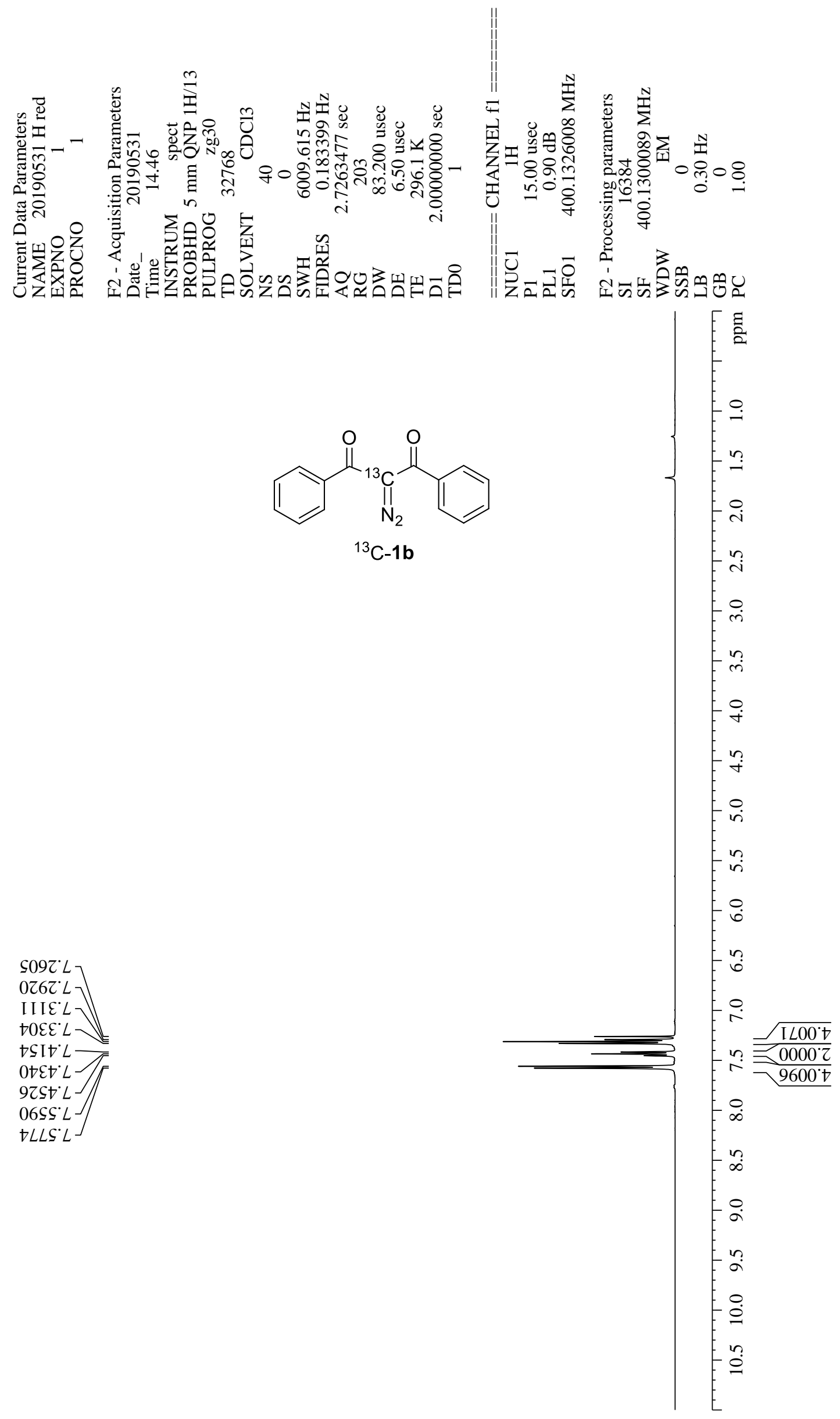



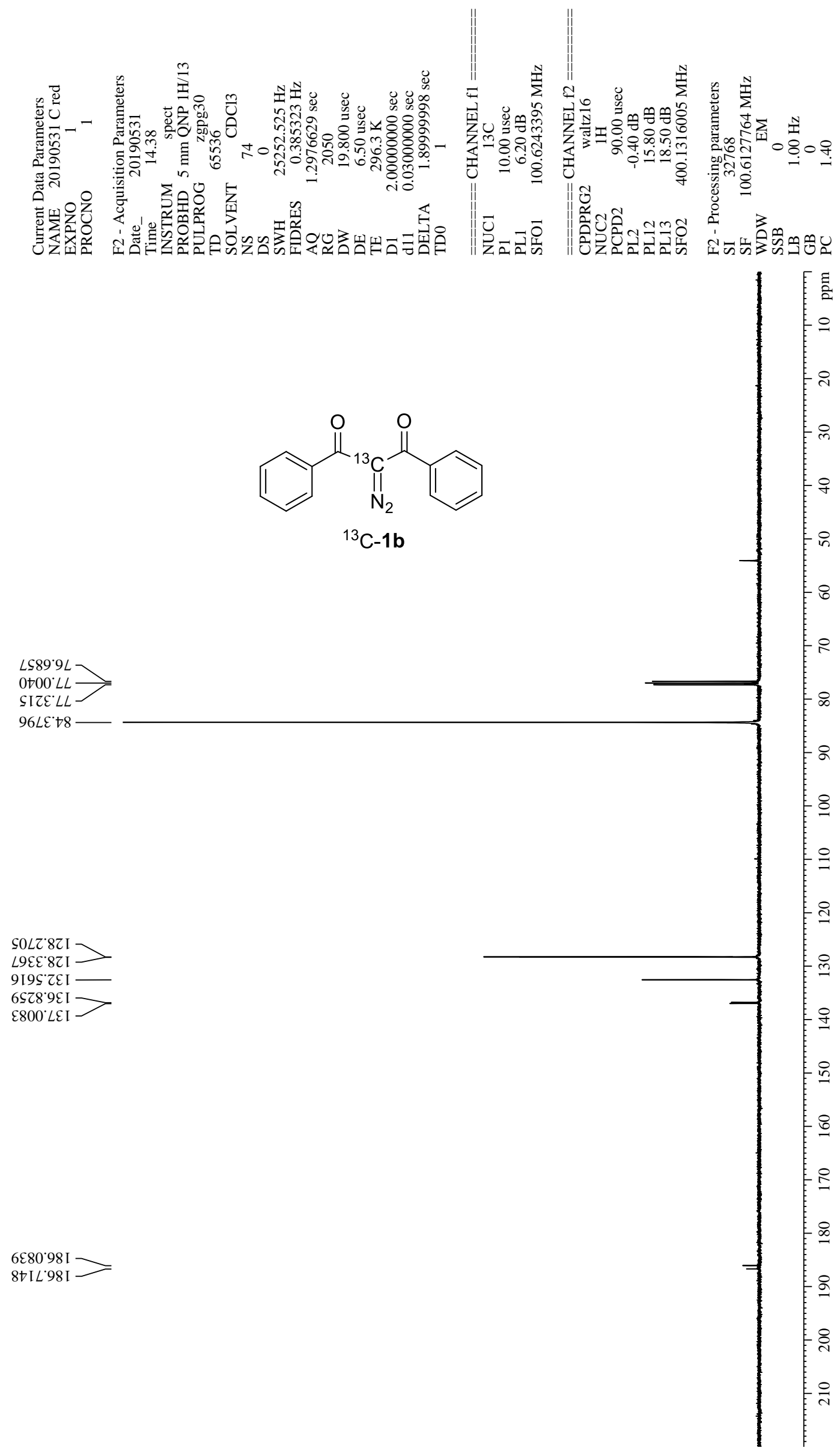


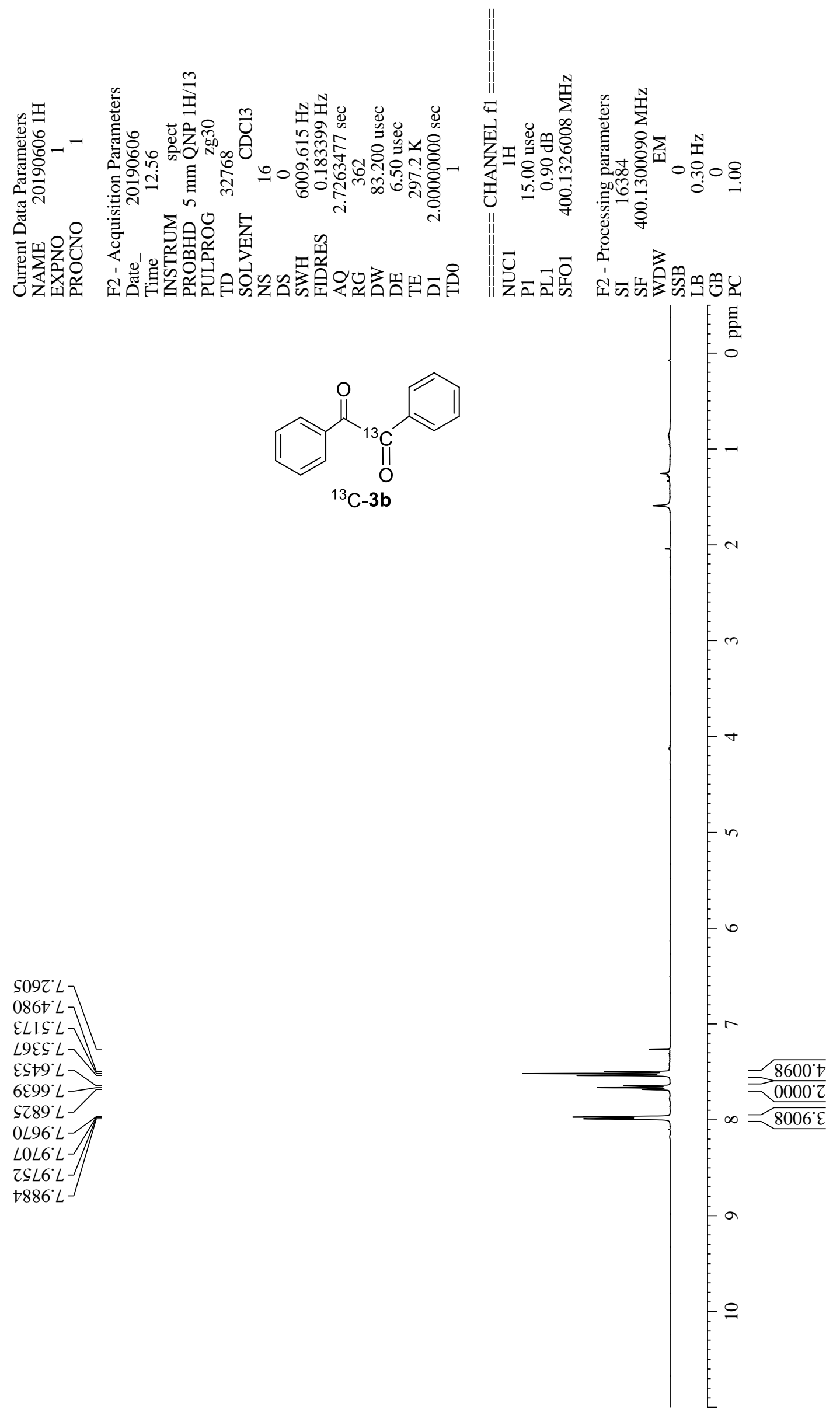




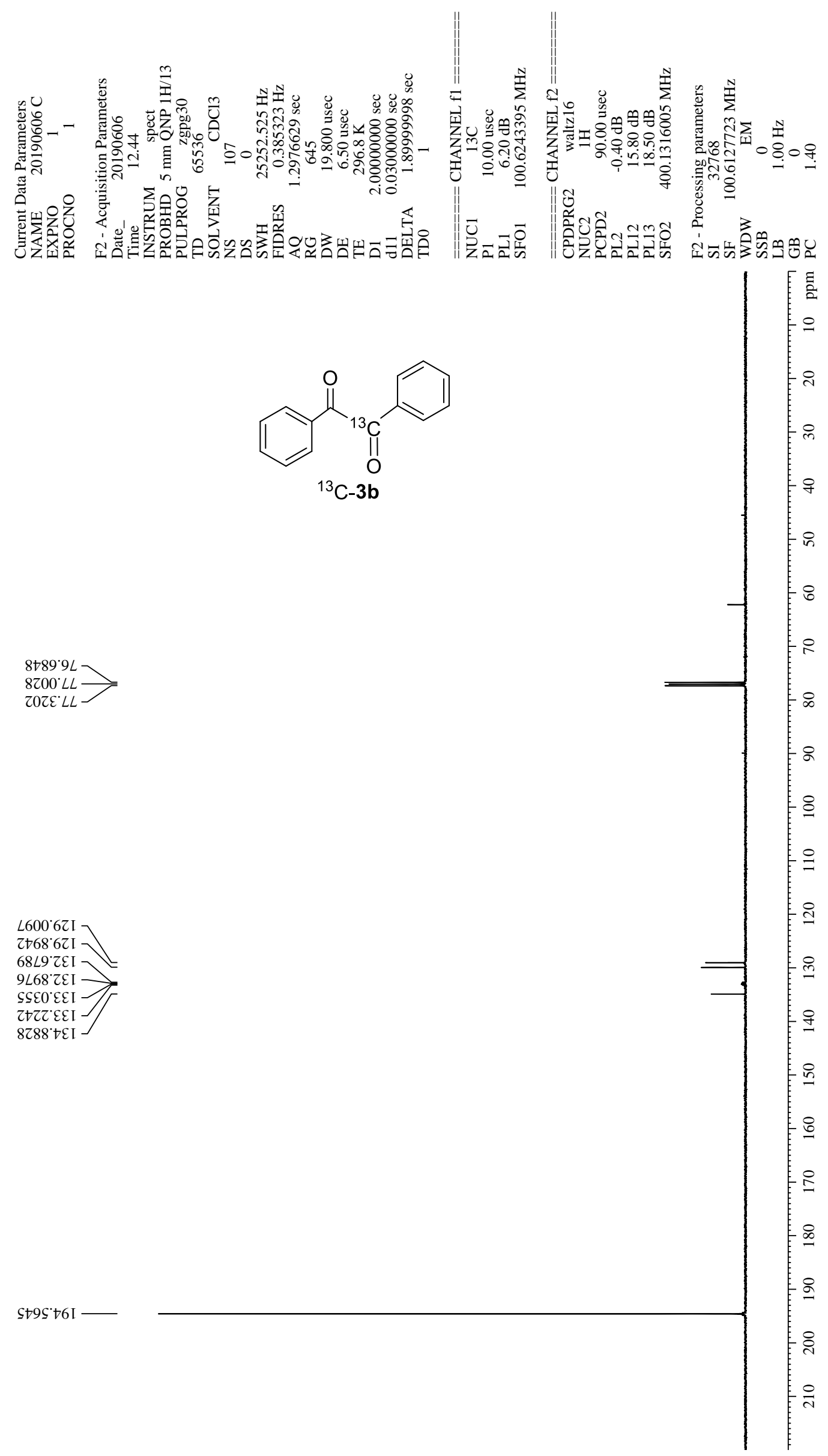




\section{6) Mass spectra of $3 b$ and ${ }^{18} \mathrm{O}-3 \mathrm{~b}$}

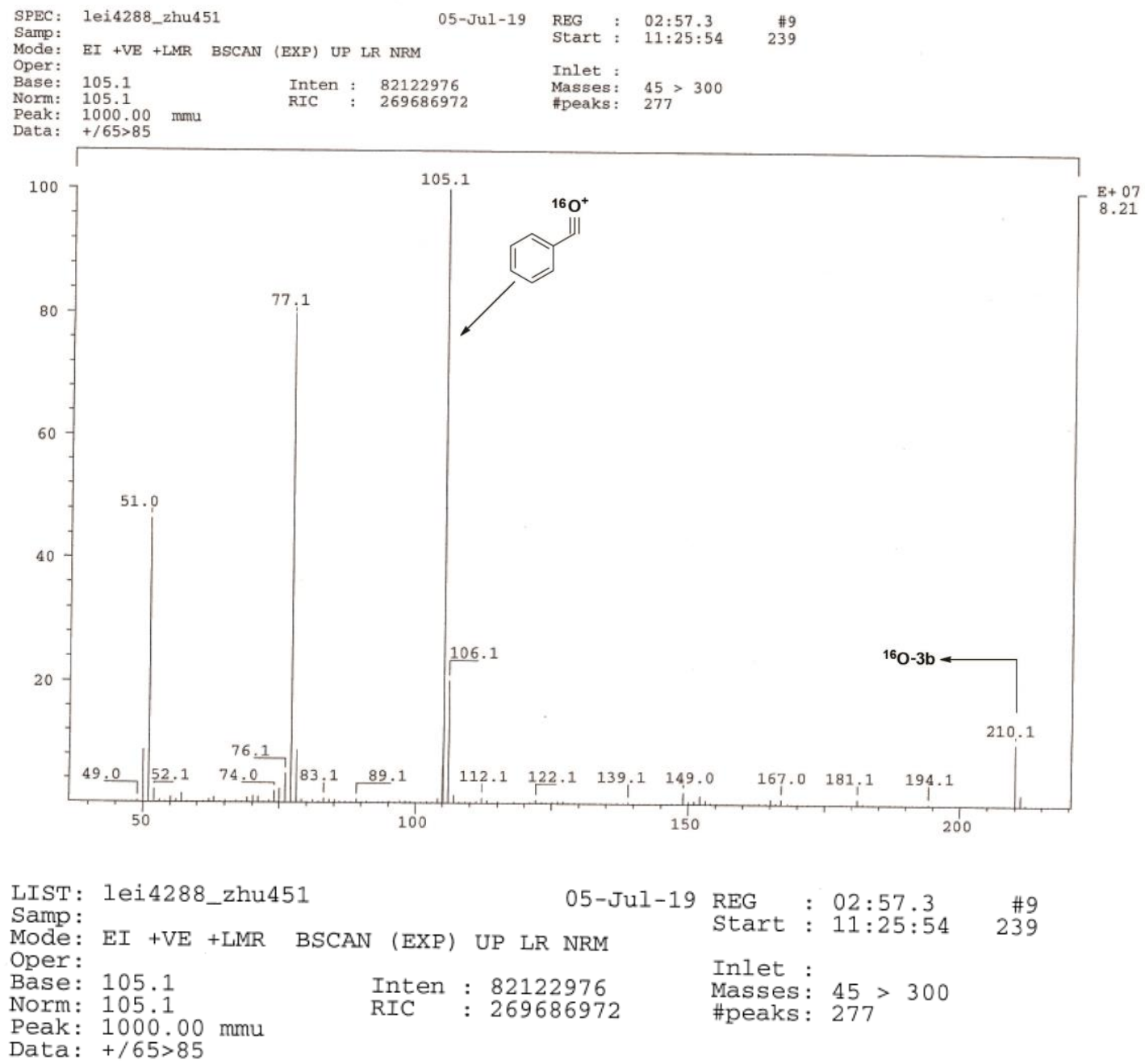

No.

181

182

183

184

185

186

187

188

189

190

191

Mass

Intensity

FRA

\%RIC

Flags

205.1

206.1

20998

0.03

0.01

0.02

16386

208.1

25029

209.1

209.7

210.1

211.1

213.1

214.1

215.1

55893

0.06

0.01

0.02

0.01

0.02

0.00

3.06

8263959
1370369

0.07

0.00

1370369

28041

1.67

0.51

0.06

0.01

6566

0.03

0.01

0.00

20304

0.02

0.01

$\#$
$\#$
$\#$
$\#$
$\#$
$\#$
$\#$
$\#$
$\#$
$\#$
$\#$
$\#$
$\#$
$\#$
$\#$




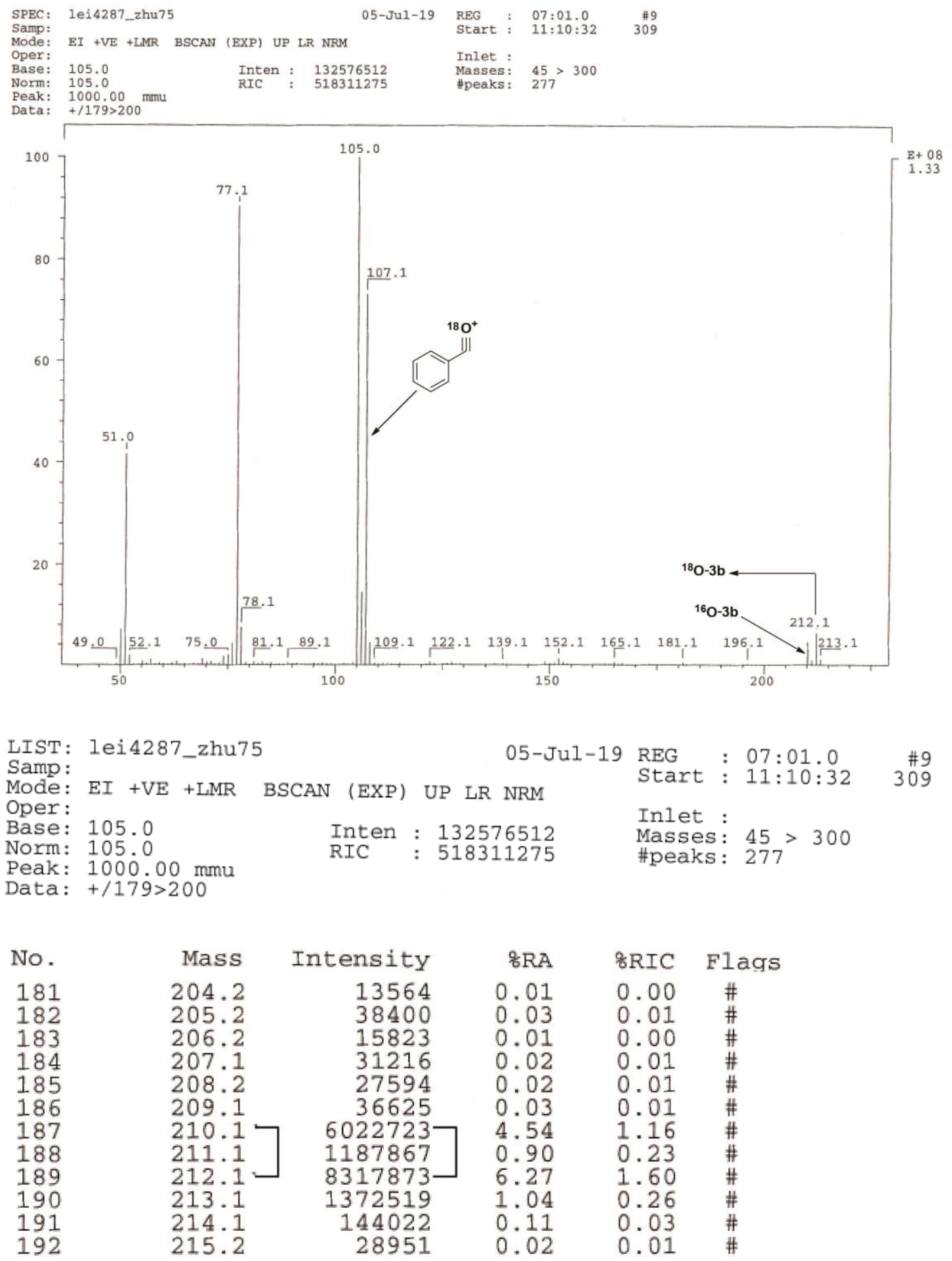


7) The ${ }^{1} \mathrm{H}$ NMR spectra of Ar- and $\mathrm{O}_{2}$-purged $\mathrm{Rh}_{2}(\mathrm{OAc})_{4}$ in $\mathrm{CDCl}_{3}$ (ref. 27)

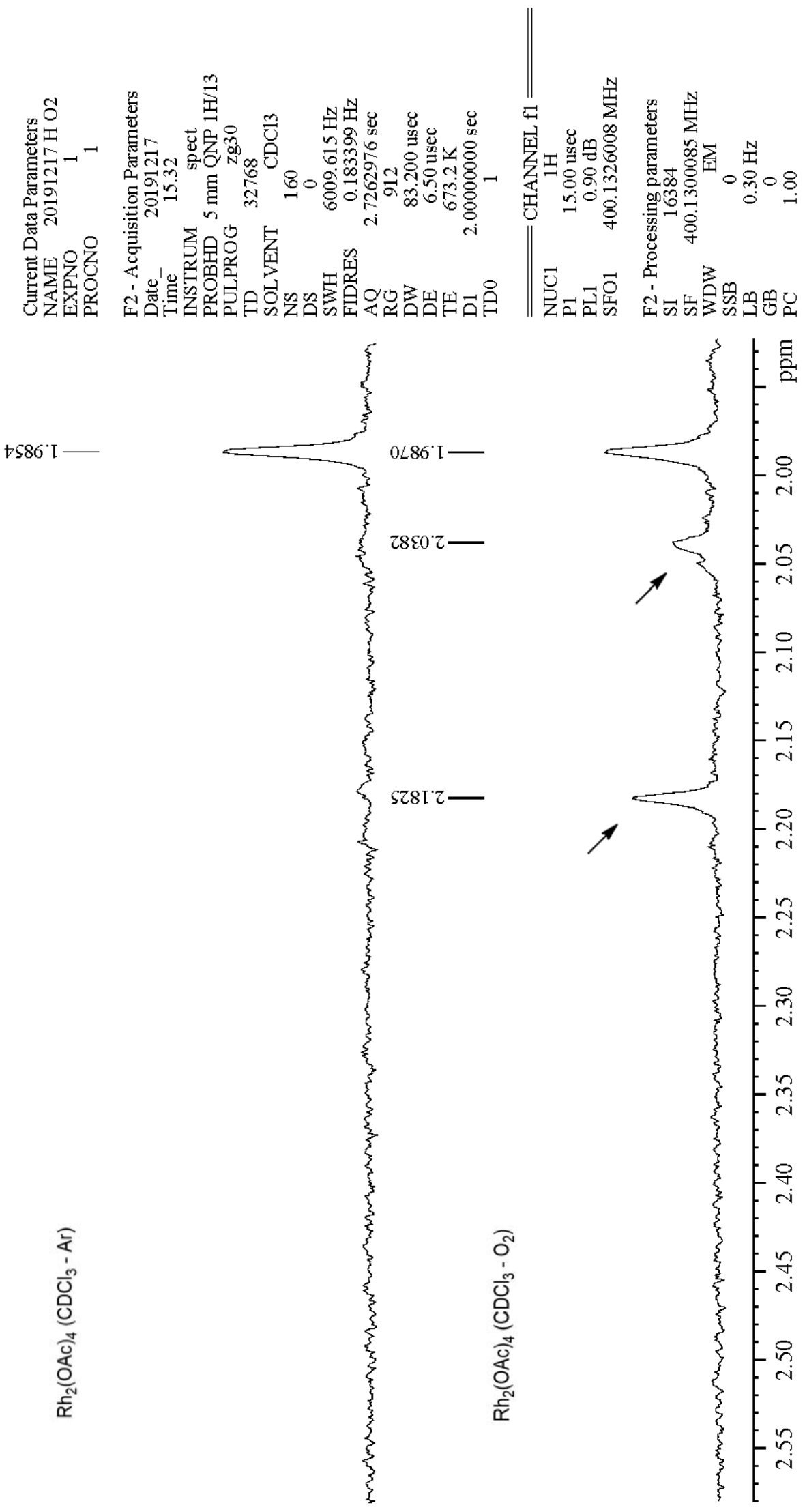




\section{8) ${ }^{1} \mathrm{H}$ and ${ }^{13} \mathrm{C}$-NMR spectra of $4 \mathrm{~b}$}
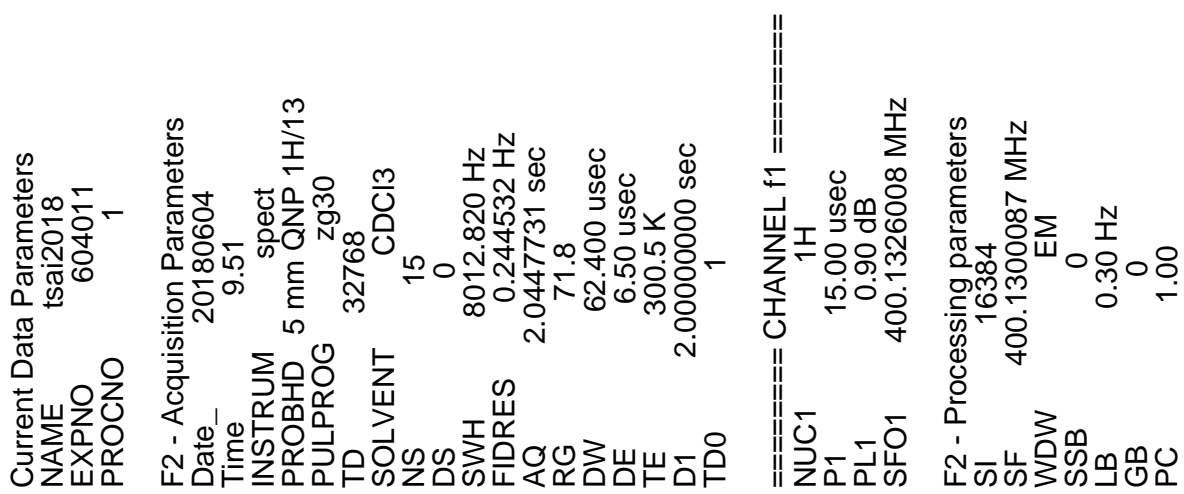<smiles>O=C(Cc1ccccc1)c1ccccc1</smiles>

4b

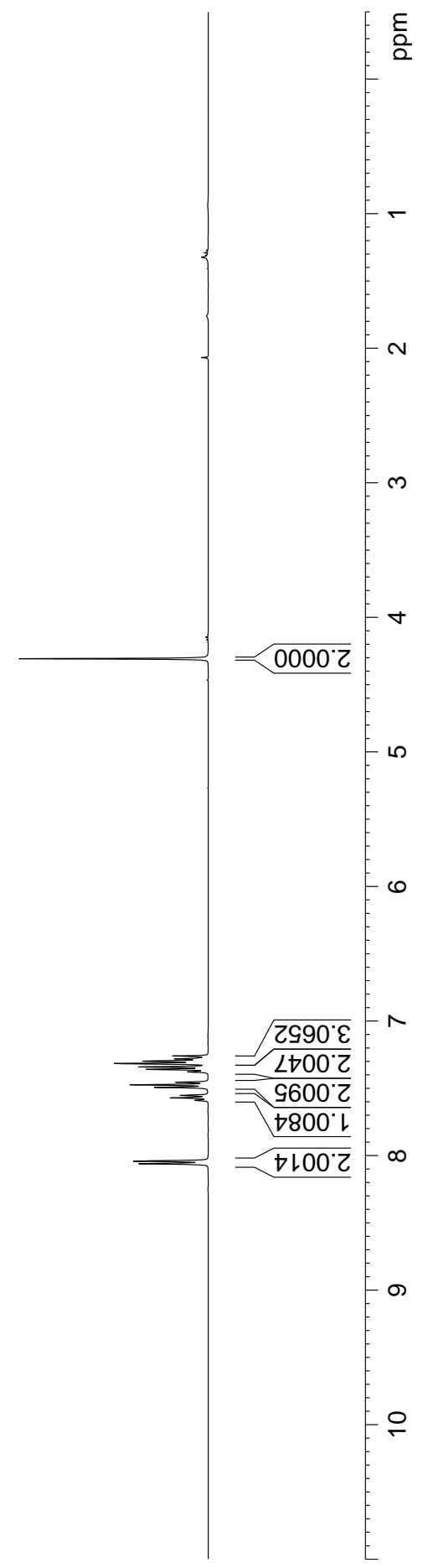




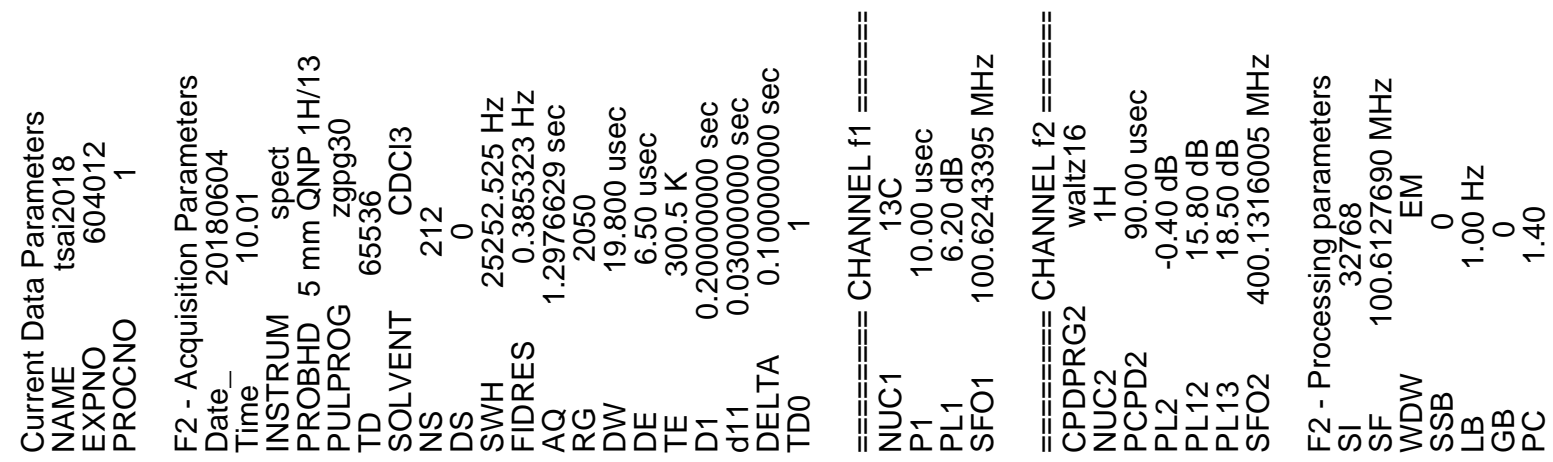
8tยG'งฺ-<smiles>O=C(Cc1ccccc1)c1ccccc1</smiles>

0E98.9L

$\varepsilon+81^{\circ} L L$

8ट8ह ' $\angle L$

266t' $L L$

†७ย6.9己เ

เ $99^{\circ} 8 \mathrm{~L}$

$0 \angle 69^{\circ} 821$

6टZL'8Z1

Stt9.62

ESOZ हEเ

0टャ9" $\bullet \varepsilon เ$

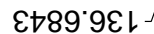

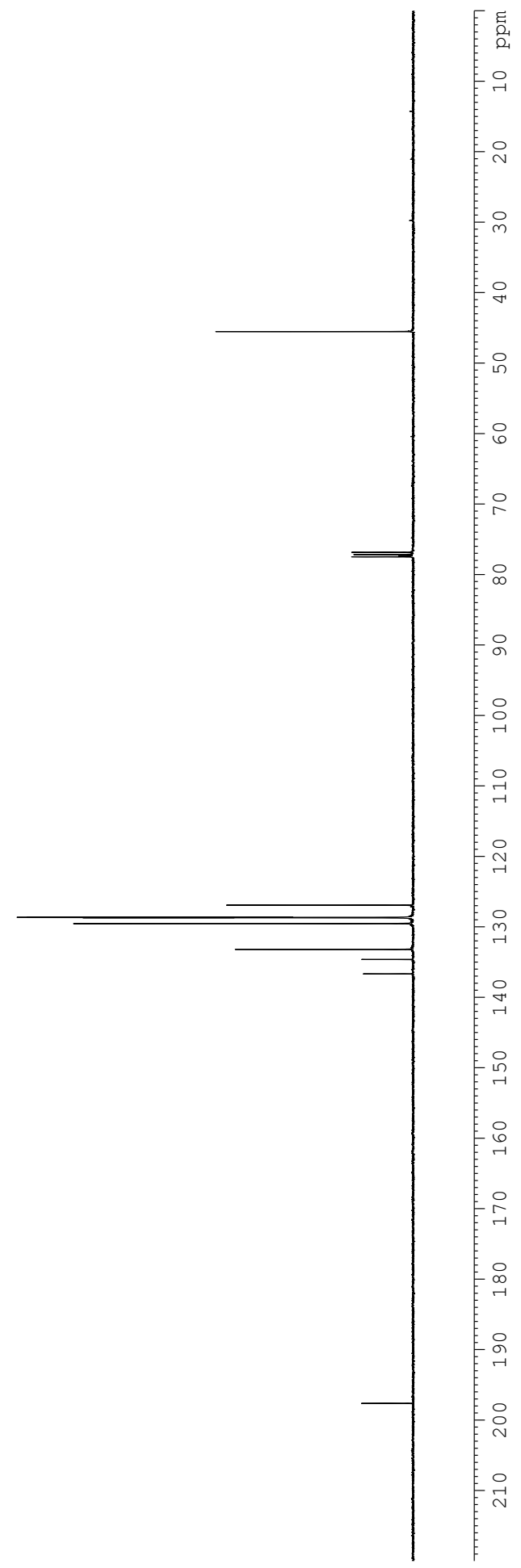


9) The image of the apparatus for preparing 3

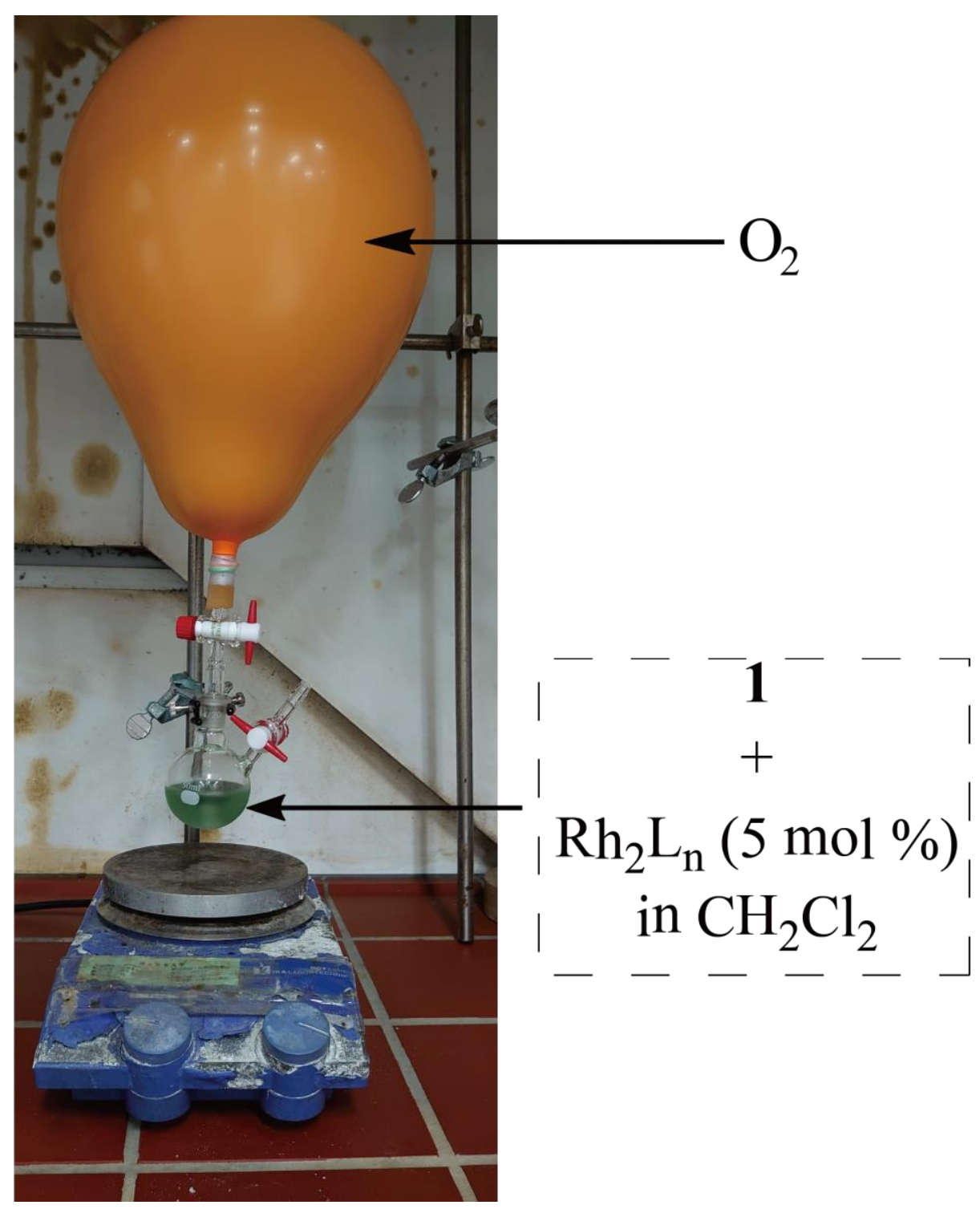

(See the Experimental Section for detailed description) 
10) Proposed mechanism for the formation of 9

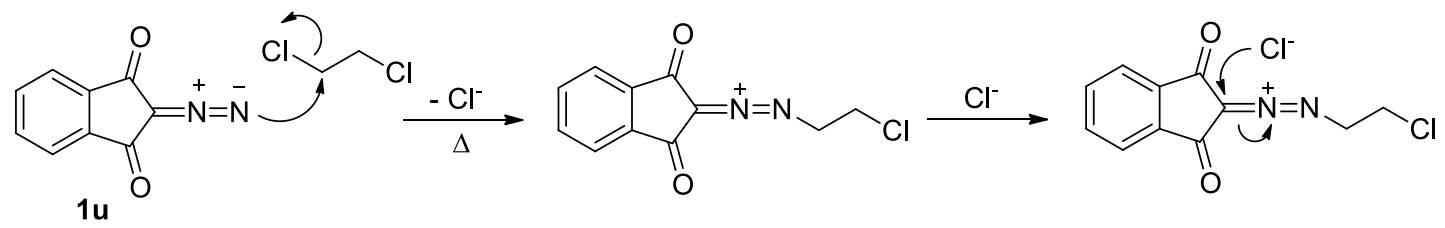<smiles>O=C1C(=O)c2ccccc2C(=O)C1=[C-]CCl</smiles> 